This is an electronic reprint of the original article. This reprint may differ from the original in pagination and typographic detail.

Author(s): Banichuk, Nikolay; Jeronen, Juha; Neittaanmäki, Pekka; Saksa, Tytti; Tuovinen, Tero

Title: $\quad$ Mechanics of Moving Materials

Year: $\quad 2014$

Version:

Please cite the original version:

Banichuk, N., Jeronen, J., Neittaanmäki, P., Saksa, T., \& Tuovinen, T. (2014).

Mechanics of Moving Materials. Springer. Solid Mechanics and Its Applications, 207. https://doi.org/10.1007/978-3-319-01745-7

All material supplied via JYX is protected by copyright and other intellectual property rights, and duplication or sale of all or part of any of the repository collections is not permitted, except that material may be duplicated by you for your research use or educational purposes in electronic or print form. You must obtain permission for any other use. Electronic or print copies may not be offered, whether for sale or otherwise to anyone who is not an authorised user. 


\title{
Mechanics of moving materials
}

\author{
N. Banichuk* J. Jeronen P. Neittaanmäki \\ T. Saksa T. Tuovinen
}

\begin{abstract}
Department of Mathematical Information Technology, University of JyVÄSKYlä, Finland

*2ND affiliation: Institute for Problems in Mechanics, Russian Academy of Sciences
\end{abstract}

May 27, 2013 


\section{Preface}

Mechanics of moving materials is currently attracting considerable attention. Interest in research in the field has grown in connection with the rapid development of process industry and precision machinery, especially in the field of paper making.

Specialized areas of investigation include dynamics and mechanical stability of travelling elastic materials, failure and reliability analysis, including modern fracture mechanics, and runnability optimization problems taking into account factors of uncertainty and incomplete data.

Problems of mechanics of moving materials have not only practical but also theoretical importance. The investigation into new types of mathematical problems is interesting in itself. It is noteworthy that there are some nonconservative stability problems, and runnability optimization problems under fracture and stability constraints with uncertainties in positioning and sizes of initial defects (cracks), for which there are no systematic techniques of investigation. The most appropriate approach to tackle the complex problem varies depending on the case.

This monograph is devoted to the exposition of new ways of formulating and solving problems of mechanics of moving materials. We present some research results concerning dynamics of travelling elastic strings, membranes, panels and plates. We study mechanical stability of axially moving elastic panels, accounting for the interaction between the structural element and its environment, such as axial potential flow. Most of the attention in this book is devoted to out-of-plane dynamics and stability analysis for isotropic and orthotropic travelling elastic and viscoelastic materials, with and without fluid-structure interaction, using analytical and numerical approaches. Also such topics as fracture and fatigue are discussed in the context of moving materials. The last part of the book deals with some runnability optimization problems with physical constraints arising from the stability and fatigue analyses including uncertainties in the parameters. The approach taken in this monograph is to proceed analytically as far as is reasonable, and only then finish the investigation numerically.

In this book, we offer a systematic and careful development of many aspects of mechanics of travelling materials, particularly for panels and plates. Some of the presented results are new, and some have appeared only in specialized journals or in conference proceedings. Some aspects of the theory presented here, such as the semi-analytical treatment of the fluid-structure interaction problem of a travelling panel, studies of spectral problems with free boundary singularities, and optimization of problem parameters under crack growth and instability constraints have not been considered before to any extent.

Important new results relate to optimization of runnability with a longevity constraint. Damage accumulation is modelled using the theory of fatigue crack growth, with the travelling material element subjected to cyclic load- 
ing. Uncertainties must be accounted for, because of incompleteness of information concerning initial crack parameters.

This book is addressed to researchers and specialists in the field, providing a view of mechanics of axially moving materials. It can also be used as literature in advanced courses on this specific topic. Considering topics related to manufacturing and processing, the book can also be applied in industrial mathematics.

We would like to express our graditude to Matti Kurki for many fruitful discussions and sharing his expertise in understanding the physical phenomena in paper making. We also express our sincere thanks to Maria Tirronen for collaboration in the research and in writing this book; Chap. 8 has been written collaboratively with Maria Tirronen. We would like to thank Metso Paper for providing Figures 1.1 and 1.2. The research presented in this book was supported by the Academy of Finland (grant no. 140221), the MASI Tekes Technology Programme, and the Jenny and Antti Wihuri Foundation.

Jyväskylä, May 2013

N. Banichuk, J. Jeronen, P. Neittaanmäki, T. Saksa and T. Tuovinen 



\section{Contents}

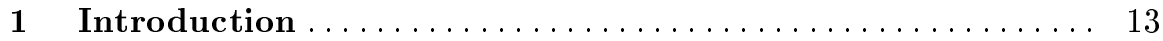

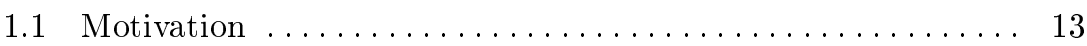

1.2 Modelling of systems with travelling continuum $\ldots \ldots \ldots \ldots \quad 15$

References................................. 19

2 Travelling strings, beams, panels, membranes and plates . 21

2.1 Out-of-plane vibrations......................... 21

2.1.1 Travelling strings ....................... 22

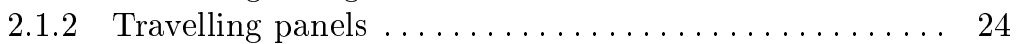

2.1.3 Travelling membranes and plates $\ldots \ldots \ldots \ldots \ldots \ldots 25$

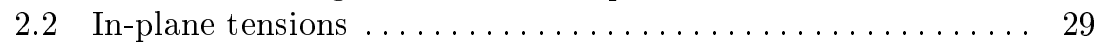

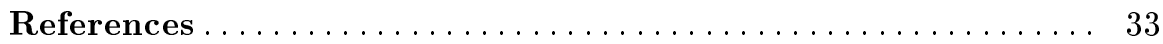

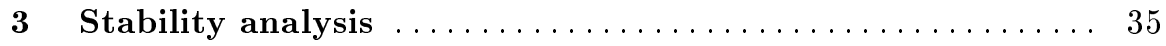

3.1 Historical view of stability investigations $\ldots \ldots \ldots \ldots \ldots \ldots 35$

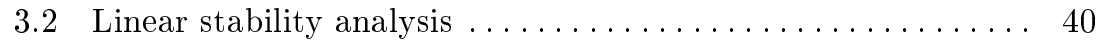

3.3 Dynamic analysis of moving membranes and plates ....... 42

3.3.1 Dynamic stability of membranes.............. 44

3.3.2 Dynamic analysis of small transverse vibrations and

elastic stability of isotropic plates $\ldots \ldots \ldots \ldots \ldots 45$

3.4 Divergence instability of isotropic plates . . . . . . . . . . 49

3.4.1 Eigenvalue problem ..................... 49

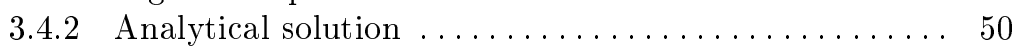

3.4.3 Properties of analytical solution ............. 53

3.5 Divergence instability of orthotropic plates $\ldots \ldots \ldots \ldots \ldots 58$

3.5.1 Eigenvalue problem ................... 59

3.5.2 Non-negativeness of eigenvalues $\ldots \ldots \ldots \ldots \ldots \ldots .61$

3.5.3 Analytical solution $\ldots \ldots \ldots \ldots \ldots \ldots \ldots \ldots \ldots \ldots .62$

3.5.4 Properties of analytical solution .............. 66 
3.5.5 Analysis of solution properties $\ldots \ldots \ldots \ldots \ldots \ldots \ldots$

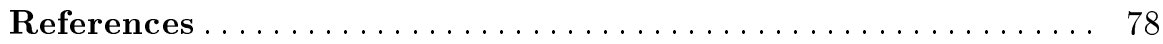

4 Non-homogeneous tension profile $\ldots \ldots \ldots \ldots \ldots \ldots \ldots 83$

4.1 Dynamic analysis of axially moving plates $\ldots \ldots \ldots \ldots \ldots 83$

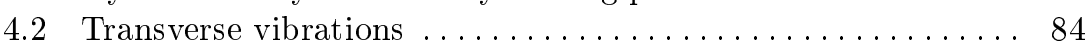

4.3 Solution of eigenvalue problem $\ldots \ldots \ldots \ldots \ldots \ldots \ldots \ldots 91$

4.3.1 Transformation to ordinary differential equation ...... 91

$4.3 .2 \quad$ Numerical analysis $\ldots \ldots \ldots \ldots \ldots \ldots \ldots \ldots \ldots . \ldots . \ldots$

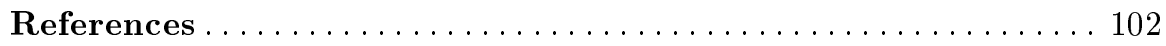

$5 \quad$ Travelling panels made of viscoelastic material ........ 103

5.1 Modelling of moving viscoelastic panels . . . . . . . . . 103

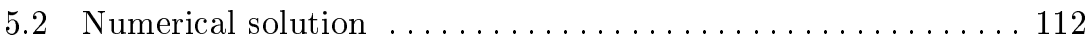

5.3 Numerical illustrations . . . . . . . . . . . . . . . . 114

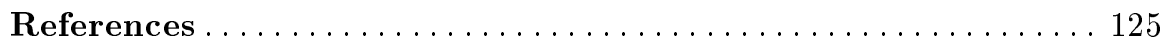

6 Travelling panels interacting with external flow $\ldots \ldots \ldots 127$

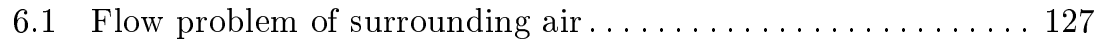

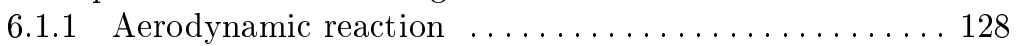

6.1 .2 Slip boundary condition . . . . . . . . . . . . . 131

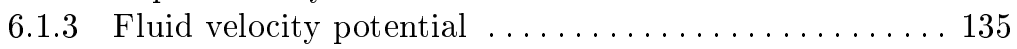

6.1.4 Complex analysis approach ................ 139

6.1.5 Properties of aerodynamic kernel . . . . . . . . . . . . . 145

6.1.6 Added-mass approximation .................. 149

6.1 .7 Notes and discussion................... 153

6.2 Behaviour of travelling panels submerged in ideal fluid ..... 154

6.2.1 Numerical considerations .................... 159

6.2 .2 Static stability analysis $\ldots \ldots \ldots \ldots \ldots \ldots \ldots \ldots \ldots \ldots \ldots$

6.2 .3 Dynamical behaviour ................... 180

6.2 .4 Eigenfrequency analysis . . . . . . . . . . . . . 190

6.2.5 Flutter problem ......................... 197

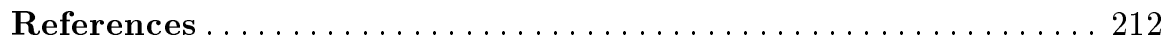

$7 \quad$ Fracture and fatigue of travelling plates $\ldots \ldots \ldots \ldots \ldots 215$

7.1 Travelling plates under fracture and instability constraints . . 2215

7.1.1 Safe range of velocities for the case of homogeneous

tension ........................... 215

7.1.2 Travelling plates under non-homogeneous tension ... . 219

7.2 Cyclic tension and constraints on longevity and instability . . 221 221

7.2.1 Fatigue crack growth and critical conditions ....... 221

7.2 .2 Numerical examples ..................... 224 
References .................................... 229

8 Some optimization problems .................... 231

8.1 Optimization of moving plates subjected to instability and

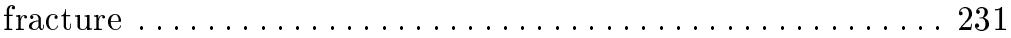

8.1.1 Optimization criterion and constraints .......... 232

8.1 .2 Finding optimal solution $\ldots \ldots \ldots \ldots \ldots \ldots \ldots \ldots 237$

8.1.3 Dependence of optimal solution on problem parameters 239

8.2 Pareto optimal solutions for good runnability .......... 243

8.2 .1 Multicriteria optimization ................. 243

8.2.2 Maximizing critical velocity and safety criterion ..... 246

8.2.3 Maximizing critical velocity and process effectiveness . 248

8.2.4 Maximizing safety and process effectiveness ........ 249

8.2 .5 Some illustrations ...................... 251

8.3 Optimization with uncertainties . ............... 254

8.3.1 Uncertainty in initial crack length . ............ 256

8.3 .2 Uncertainty in tension $\ldots \ldots \ldots \ldots \ldots \ldots \ldots \ldots \ldots . \ldots \ldots$

References ................................... 266

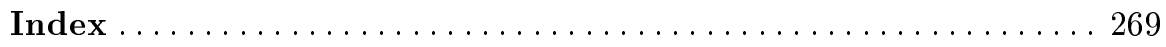




\section{List of symbols}

Ordering is alphabetical, case-sensitive, with Greek letters placed according to their name in the Latin alphabet (e.g. $\Phi \rightarrow$ Phi, $\varphi \rightarrow$ phi, $\Pi \rightarrow \mathrm{Pi}$ ).

Example: $P, P(x), P_{1}, P_{12}, P_{Q}, P_{i j}, P^{j}, P_{n}, p, p_{j}, \Phi, \Phi_{i}, \varphi, \Pi$.

$A^{\mathrm{a}} \quad$ constant related to antisymmetric solution (Chap. 3 ).

$A_{j n} \quad$ local acceleration matrix in fluid-structure problem (Chap. 6).

$A^{\mathrm{s}} \quad$ constant related to symmetric solution (Chap. 3 ).

$a \quad$ length of edge crack (Chaps. 7 and 8).

$a_{j n} \quad$ fluid-structure interaction matrix analogous to local acceleration in fluid-structure problem (Chap. 6).

$\alpha \quad$ linear tension profile slope constant (Chap. 4), see (2.51). Unit $[\alpha]=\mathrm{N} / \mathrm{m}^{2}$. Dimensionless problem parameter (Chap. 5), see (5.29). Dimensionless dynamic time scale (Chap. 6), see (6.66). Weight function (Chap. 8), see (8.51).

$B^{\mathrm{a}} \quad$ constant related to antisymmetric solution (Chap. 3).

$B_{j n} \quad$ Coriolis matrix in fluid-structure problem (Chap. 6).

$B^{\mathrm{s}} \quad$ constant related to symmetric solution (Chap. 3 ).

$b$ half-width of plate; in the width direction, the plate occupies the interval $y \in[-b, b]$.

$b_{j n} \quad$ fluid-structure interaction matrix analogous to Coriolis acceleration in fluid-structure problem (Chap. 6).

$\beta \quad$ dimensionless bending rigidity of panel (Chap. 6), see (6.63). Geometric factor for stress intensity factor (Chaps. 7 and 8), see (7.7).

$\beta_{j} \quad$ (where $j=1,2$ ) mechanical parameters in free edge boundary conditions of the plate. See (2.23) on p. 28.

$C \quad$ critical velocity of the travelling ideal string, $C=\sqrt{T / m}$. The unit $[C]=\mathrm{m} / \mathrm{s}$. The quantity $C$ is used as a scaling constant for nondimensionalizing velocities. In Chap. 7, material constant in Paris law.

$C_{\mathrm{M}} \quad$ weighting factor for productivity function $J_{\mathrm{M}}$ (Chap. 8), see (8.35).

$C_{\mathrm{N}} \quad$ weighting factor for safety function $J_{\mathrm{N}}$ (Chap. 8), see (8.35).

$C_{\mathrm{V}} \quad$ weighting factor for critical velocity function $J_{\mathrm{V}}$ (Chap. 8), see (8.35).

$C_{i j} \quad$ (where $\left.i, j=1,2,6\right)$ elastic moduli. Note symmetry $C_{12}=C_{21}$.

$C_{j n} \quad$ centripetal matrix in fluid-structure problem (Chap. 6).

$c \quad$ dimensionless velocity of the panel, $c=V_{0} / C$.

$c_{j n} \quad$ fluid-structure interaction matrix analogous to centripetal acceleration in fluid-structure problem (Chap. 6).

$c_{1} \quad$ probability distribution parameter (Chap. 8), see (8.61).

$c_{\mathrm{t}} \quad$ probability distribution parameter (Chap. 8), see (8.71).

$\chi \quad$ auxiliary function for stream function boundary data (Chap. 6).

$D \quad$ bending rigidity. For the panel, this is the cylindrical bending rigidity $D=E h^{3} /\left(12\left(1-\nu^{2}\right)\right)$. Unit $[D]=\mathrm{N} \mathrm{m}$. 
$D_{0} \quad$ scaling constant for nondimensionalization of bending rigidity, called the characteristic bending rigidity. Unit $\left[D_{0}\right]=\mathrm{N} \mathrm{m}$.

$D_{j} \quad$ (where $j=1,2,3$ ) orthotropic bending rigidity. $D_{1}$ is related to the axial direction $x, D_{2}$ to the cross (width) direction $y$, and $D_{3}$ to the coupling term between the two.

$D_{j n} \quad$ bending matrix in fluid-structure problem (Chap. 6).

$\Delta T \quad$ parameter of maximum tension variation (Chap. 7).

$\delta \quad$ small positive real number (Chap. 5). Dirac delta distribution (Chap. 6).

$E \quad$ Young's modulus of isotropic plate or panel, $[E]=\mathrm{N} / \mathrm{m}^{2}$.

$E(\theta) \quad$ Expectation value for the random part of uncertain tension (Chap. 8).

$E(\xi) \quad$ Expectation value for the crack length (Chap. 8).

$E_{1} \quad$ Young's modulus of orthotropic plate in axial direction, $\left[E_{1}\right]=$ $\mathrm{N} / \mathrm{m}^{2}$.

$E_{2} \quad$ Young's modulus of orthotropic plate in cross (width) direction, $\left[E_{2}\right]=\mathrm{N} / \mathrm{m}^{2}$.

$\varepsilon_{x x} \quad$ axial strain component. $\varepsilon_{x x}=\partial u / \partial x$.

$\varepsilon_{y y} \quad$ width-directional strain component. $\varepsilon_{y y}=\partial v / \partial y$.

$\eta \quad$ dimensionless cross-direction coordinate (Chaps. 3, 4). Viscous damping coefficient (Chap. 5). A point of the complex plane (Chap. 6).

$F_{\theta} \quad$ cumulative distribution function of tension random variable $\theta$ (Chap. 8), see (8.71).

$F_{\xi} \quad$ cumulative distribution function of crack length random variable $\xi$ (Chap. 8), see (8.62).

$f(\eta) \quad$ auxiliary function of cross-directional buckling shape (Chaps. 3, 4).

$f(\xi) \quad$ auxiliary function (Chap. 6).

$f_{n}(t) \quad$ or $f_{j}(t)$; coefficient function of $n$th (resp. $j$ th) term in Galerkin series (Chap. 6).

$f_{\xi} \quad$ probability density function of crack length random variable $\xi$ (Chap. 8).

G discretized external load (Chap. 6).

$\mathcal{G}$ external load function (Chap. 6).

$G_{12}$ in-plane shear modulus of orthotropic plate, $\left[G_{12}\right]=\mathrm{N} / \mathrm{m}^{2}$. Unless otherwise specified, it is taken as an independent material parameter.

$G_{\mathrm{C}} \quad$ strain energy release rate (Chaps. 7 and 8).

$G_{\mathrm{H}}$ geometric average shear modulus for orthotropic materials, $G_{\mathrm{H}} \equiv$ $\sqrt{E_{1} E_{2}} /\left(2\left(1+\sqrt{\nu_{12} \nu_{21}}\right)\right)$.

$g \quad$ External force (Chap. 6). Dimensionless parameter (Chap. 8), see (8.21).

$g(\xi) \quad$ auxiliary function (Chap. 8), see (8.52) and explanation that follows.

$g(y) \quad$ tension profile function (Chap. 4).

$g_{1}(x) \quad$ initial condition for position (Chap. 6).

$g_{2}(x)$ initial condition for transverse (out-of-plane) velocity (Chap. 6). 
$\Gamma \quad$ domain boundary. In Chap. 6, circulation of flow.

$\gamma \quad$ square root of eigenvalue of static stability problem, $\lambda=\gamma^{2}$ (Chaps. 3, 4, 7 and 8). Dimensionless problem parameter (Chap. 5), see (5.29). Dimensionless fluid density (Chap. 6), see (6.62). Also, in Chap. $6, \gamma(x, t)$ is the expression of the airflow boundary condition on the panel surface.

$\gamma_{x y} \quad$ shear strain. $\gamma_{x y}=\partial u / \partial y+\partial v / \partial x$.

$H_{j} \quad$ (where $j=1,2,3$ ) dimensionless bending rigidity. $H_{j}=D_{j} / D_{0}$.

$h \quad$ plate or panel thickness, $[h]=\mathrm{m}$.

I identity matrix.

$i \quad$ imaginary unit, $i=\sqrt{-1}$. (Unless otherwise specified.)

$J \quad$ productivity criterion (Chap. 8).

$J_{\mathrm{M}} \quad$ productivity function (Chap. 8), see (8.31).

$J_{\mathrm{N}} \quad$ safety function related to critical number of cycles (Chap. 8), see (8.30).

$J_{\mathrm{V}} \quad$ critical velocity function (Chap. 8), see (8.29).

$K \quad$ stress intensity factor (Chaps. 7 and 8 ).

$\mathcal{K}$ integro-differential operator for fluid-structure interaction (Chap. $6)$.

$K_{\mathrm{C}} \quad$ fracture toughness (Chaps. 7 and 8 ).

$k \quad$ in Chap. 7, material constant in Paris law.

$k(y) \quad$ tension profile function, specifying axial tension in terms of the plate width coordinate at the rollers. See (2.29).

$\kappa_{ \pm} \quad$ solution parameter (Chaps. 3, 4, 7 and 8). See (3.48) and (3.102).

$\varkappa \quad$ eigenvalue (exact meaning is context-dependent).

$\mathcal{L} \quad$ differential operator of vacuum problem (Chap. 6).

$\mathcal{L}_{0}(w)$ bending operator (see index).

$\mathcal{L}^{\mathrm{B}}(w)$ bending operator (see index).

$\mathcal{L}^{\mathrm{M}}(w)$ membrane operator (see index).

$\ell \quad$ free span length parameter. For the panel and ideal string, the free span is taken to be $x \in[-\ell, \ell]$. For the plate and membrane, the length of the free span is taken as $x \in[0, \ell]$. Unit $[\ell]=\mathrm{m}$.

$\Lambda$ auxiliary function for defining aerodynamic kernel (Chap. 6), see (6.39).

$\lambda \quad$ eigenvalue (exact meaning is context-dependent).

$M \quad$ bending moment (Chap. 5).

M discrete problem matrix, complete (exact meaning is context-dependent).

$\mathbf{M}_{j} \quad$ discrete problem matrix, block component (exact meaning is contextdependent).

$m$ for the plate and panel, mass per unit area, $[m]=\mathrm{kg} / \mathrm{m}^{2}$. For the ideal string, $m$ is the mass per unit length, $[m]=\mathrm{kg} / \mathrm{m}$.

$m_{\mathrm{a}} \quad$ added mass (Chap. 6, discussion of added-mass approximation).

$\mu \quad$ Scaled aspect ratio, $\mu=(1 / \pi)(\ell / b)$ (Chaps. 3, 4, 7 and 8). Poisson ratio for viscosity (Chap. 5). Auxiliary mean value for added-mass approximation (Chap. 6). 
$N \quad$ aerodynamic kernel function (Chap. 6), see (6.39). Cumulative distribution function of the standard normal distribution (Chap. 8), see (8.63).

n unit normal vector.

$n \quad$ cycle number (Chaps. 7 and 8 ).

$n_{0} \quad$ number of basis functions in discrete approximation (Chap. 6).

$n_{\mathrm{C}} \quad$ critical number of cycles (Chaps. 7 and 8).

$\nu \quad$ Poisson ratio of isotropic plate or panel.

$\nu_{12} \quad$ Poisson ratio for orthotropic plate. When stretched along axis $1, \nu_{12}$ is the contraction factor along axis 2 .

$\nu_{21} \quad$ Poisson ratio for orthotropic plate. When stretched along axis 2, $\nu_{21}$ is the contraction factor along axis 1 .

$\Omega \quad$ domain of the governing equation (connected open set in $\mathbb{R}^{d}$ ). In Chaps. 2-4, 7 and 8, the definition is $\Omega \equiv\left\{(x, y) \in \mathbb{R}^{2} \mid 0<x<\right.$ $\ell,-b<y<b\}$. In Sect. 6.1, $\Omega$ refers to the domain of the flow problem, $\Omega \equiv \mathbb{C} \backslash S$, where $S$ is the linearized representation of the panel, $S \equiv\{\zeta \in \mathbb{C} \mid \zeta=x+i z,-1 \leq x \leq 1, z=0\}$. In Sect. 6.2, $\Omega$ refers to the domain of the dimensionless dynamical equation of the panel, $\Omega \equiv\{x \in \mathbb{R} \mid-1<x<1\}$.

$\Omega_{i} \quad$ subdomain between the $i$ th pair of rollers (Chap. 8), defined as $\Omega_{i} \equiv\left\{(x, y) \in \mathbb{R}^{2} \mid i \ell<x<(i+1) \ell,-b<y<b\right\}$, where $i=0,1,2, \ldots$.

$P(x) \quad$ probability that condition $x$ is true (Chap. 8).

$p \quad$ pressure of fluid (Chap. 6). Probability of fracture (Chap. 8).

$\Phi$ transcendental auxiliary function related to analytical solution of static stability problem (Chaps. 3, 7 and 8). Velocity potential of fluid flow (Chap. 6).

$\varphi \quad$ velocity potential of disturbance due to obstacle (Chap. 6).

$\Pi \quad$ complex velocity potential (Chap. 6).

$\Psi \quad$ algebraic auxiliary function related to analytical solution of static stability problem (Chaps. 3, 7 and 8). Stream function of fluid flow (Chap. 6).

$\Psi_{n} \quad$ basis function for Galerkin representation of solution (Chap. 6).

$q_{1} \quad$ admissible probability of fracture (Chap. 8).

$q_{2} \quad$ admissible probability of instability (Chap. 8).

$q_{\mathrm{f}} \quad$ aerodynamic reaction pressure (see index).

$\rho_{\mathrm{f}} \quad$ density of surrounding fluid. Unit $\left[\rho_{\mathrm{f}}\right]=\mathrm{kg} / \mathrm{m}^{3}$.

$S \quad$ linearized surface of panel (Chap. 6).

$s \quad$ stability exponent in linear stability analysis (see index).

$s_{1} \quad$ probability distribution parameter (Chap. 8), see (8.61).

$\sigma_{x x} \quad$ axial stress for the plate. Unit $\left[\sigma_{x x}\right]=\mathrm{N} / \mathrm{m}^{2}$.

$\sigma_{x y} \quad$ shear stress for the plate. Unit $\left[\sigma_{x y}\right]=\mathrm{N} / \mathrm{m}^{2}$.

$\sigma_{y y} \quad$ cross-directional (width-directional) stress for the plate. Unit $\left[\sigma_{y y}\right]=$ $\mathrm{N} / \mathrm{m}^{2}$. 
$T$ tension. For the plate and panel, tension is defined in terms of the stress as $T=h \sigma$, and the unit is $[T]=\mathrm{N} / \mathrm{m}$. For the ideal string, $T$ is the axial tension force, with unit $[T]=\mathrm{N}$.

$T_{0} \quad$ constant (homogeneous) tension.

$T_{0}^{\mathrm{M}} \quad$ maximum admissible tension (Chap. 8).

$T_{x x} \quad$ axial tension for the plate, $T_{x x}=h \sigma_{x x}$. Unit $\left[T_{x x}\right]=\mathrm{N} / \mathrm{m}$.

$T_{x y} \quad$ in-plane shear tension for the plate, $T_{x y}=h \sigma_{x y}$. Unit $\left[T_{x y}\right]=\mathrm{N} / \mathrm{m}$.

$T_{y y} \quad$ cross-directional (width-directional) tension for the plate, $T_{y y}=$ $h \sigma_{y y}$. Unit $\left[T_{y y}\right]=\mathrm{N} / \mathrm{m}$.

$t$ time coordinate.

t unit tangent vector (Chap. 6).

$t_{\mathrm{f}} \quad$ total time for process (Chap. 8).

$t_{\mathrm{R}} \quad$ retardation time constant (Chap. 5).

$\tau \quad$ scaling constant for nondimensionalization of time coordinate,called the characteristic time. $[\tau]=\mathrm{s}$. In Chap. 8 , cycle time period.

$\theta \quad$ dimensionless fluid velocity, $\theta=v_{\infty} / C$. In Chap. 8 , random variable for uncertain tension.

U displacement field of the panel (Chap. 6).

$u \quad$ axial ( $x$-directional) displacement. $[u]=\mathrm{m}$.

$\mathbf{u} \quad$ auxiliary discrete vector of unknowns.

$\Upsilon \quad$ Airy stress function; see (2.42) on p. 32. In Chap. 5, viscous analogue of bending rigidity.

$V_{0} \quad$ axial velocity of the plate, panel or string. $\left[V_{0}\right]=\mathrm{m} / \mathrm{s}$.

$V_{0}^{\text {cr }} \quad$ critical velocity of elastic instability of the travelling plate (Chap. $8)$, see (8.12).

$v \quad$ cross-directional ( $y$-directional) displacement. $[v]=\mathrm{m}$.

$v_{\infty} \quad$ free-stream velocity of surrounding fluid. $\left[v_{\infty}\right]=\mathrm{m} / \mathrm{s}$.

$\mathbf{v}_{\mathrm{f}} \quad$ velocity field of fluid (Chap. 6).

$W \quad$ transverse ( $z$-directional, out-of-plane) displacement. Used for the space part of the time-harmonic solution, and for the buckling (divergence) mode. transverse ( $z$-directional, out-of-plane) displacement, dimensional or dimensionless depending on context. Unit of dimensional displacement $[w]=\mathrm{m}$. auxiliary discrete vector of unknowns. axial space coordinate in Eulerian (stationary) reference frame. in Chap. 6, axial space coordinate in Lagrangean (co-moving) reference frame. Also used as a dummy variable for integration in the same chapter. In Chap. 8, positive valued random variable describing the crack length.

y cross-directional (width) space coordinate.

y auxiliary discrete vector of unknowns.

$z \quad$ transverse (out-of-plane) space coordinate. 


\title{
Chapter 1 Introduction
}

\begin{abstract}
In this introductory chapter, the idea of moving materials is briefly introduced, and the theoretical framework that will be discussed in this book is linked to the manufacturing process of paper products. The content and structure of the book is also presented, outlining the topics handled in each chapter. The main focus of the book is on dynamics and stability of moving elastic and viscoelastic materials, with and without fluid-structure interaction, using linear models for out-of-plane behaviour in the small displacement regime. However, also such topics as fracture and fatigue are discussed in the context of moving materials. The last part of the book deals with some runnability optimization problems with physical constraints arising from stability and fatigue analyses including uncertainties in the parameters.
\end{abstract}

\subsection{Motivation}

The impetus for this book came from the industry where the mechanics of moving materials is a highly interesting problem. Especially paper production, for which moving materials are an essential topic, is an important segment of Finnish industry with a long tradition. When viewed on the global level, paper production is of course not limited only to Finland, but is a worldwide industry, with for example Canada, China, Germany, Japan, Russian Federation, United Kingdom, United States, and Sweden as producers of paper (for a comparison of major paper producers see, e.g., Kenny 2006; PricewaterhouseCoopers LLP 2012).

What, then, is a moving material? Stated briefly, in problems of moving materials, the domain of interest is a stationary control volume, through which the moving material flows. The material flow can be steady-state or fully dynamic. The moving material is typically a solid, thus differentiating the topic of moving materials from fluid mechanics. Nevertheless, from the 
viewpoint of classical mechanics, moving materials are more closely related to flowing fluids than to structural mechanics.

Traditional solid objects in motion, such as aeroplane wings or rocket coating plates, are excluded from moving materials, because they can be analyzed as stationary objects in a Lagrangean (co-moving) frame of reference by using the principle of Galilean relativity. As far as the physics is concerned, a wing moving through stationary air is equivalent to a stationary wing subjected to oncoming wind.

For moving materials such a change of viewpoint is usually not possible. The motion of the moving material, as it flows through the control volume of interest, prohibits convenient treatment in a purely Lagrangean frame of reference as would be done in classical structural mechanics, because in the Lagrangean frame, the boundaries of the control volume are in motion. It becomes more convenient to look at the situation in the Eulerian (stationary) frame, where the material flows, but the boundaries of the control volume remain stationary.

The overall motion of the material in the Eulerian frame where it is analyzed changes the nature of the problem, introducing new challenges. As is known, changing the coordinate system will not change the behaviour of the physical system under study, but it must be kept in mind that the theory of structural mechanics has practically always been studied in a Lagrangean frame of reference, and for moving materials, one needs a different viewpoint. The key factor is the inertia of the moving material, which gives rise to inertial effects when its motion is viewed in the Eulerian frame.

Why study the behaviour of moving materials? Practical applications for the mechanics of moving materials are, e.g., the processing of paper or steel, fabric, rubber or some other continous material; and looping systems such as band saws, transmission cables, and conveyor and timing belts. Also tape drives, and if rotating motion is allowed, optical disc and hard disk drives are possible applications. In this book, the parameters and material properties have been chosen mainly with paper production in mind. However, the theory is not anyhow restricted to this area, but can be utilized for many other similar applications with careful consideration of the underlying physics.

Looking inside a paper machine, one sees that the paper that is being manufactured travels as a thin sheet. Viewed close up, this paper web is made of a three-dimensional web of fibers, but at the macroscopic scale it appears as a uniform sheet. The sheet is very thin; typical thicknesses vary from $0.1 \mathrm{~mm}$ (office paper) to $1 \mathrm{~mm}$ (cardboard). All paper machines contain open draws, where the paper web momentarily travels without mechanical support, while it is moving from one supporting roller to another. In any practical paper machine there is always at least one open draw, in the transfer from the press nip, which squeezes out most of the water, to the dryer section. Also, if the dryer section is of the classical heated cylinder type, which has been used for over a century, an open draw exists between each successive pair of cylinders (see Karlsson 2000). When a thin sheet of material travels 
without mechanical support, it is subject to destabilizing effects such as the centrifugal effect due to inertia of the axially moving material particles, and aerodynamic reaction forces due to the surrounding air.

This book deals with the stability of the web, the physical environment overall, and critical points where the system may fail. The productivity of a paper mill is strongly dependent on the efficiency and reliability of the running web. If it is possible to increase the velocity of the web, production becomes higher and there will be more paper to sell. Increasing the velocity means also that one must increase tension of the web, in order to retain mechanical stability. However, increasing the tension in the web increases the probability of web breaks due to cracks and other defects, which causes a loss in the total efficiency of the system. One of our targets in this book is to find an optimal solution for this problem.

\subsection{Modelling of systems with travelling continuum}

The aim of the research, on which this book is based, has been to create a mathematical model, which simplifies the problems of moving materials sufficiently, while still providing an understanding of the phenomena, qualitatively and quantitatively. Concentrating on the specific application of paper production, a major issue, and a known challenge, has been the fact that measurements inside the paper machine are either highly expensive, or often impossible. Furthermore, a fully detailed model of the whole paper machine is not tractable, even using the methods and computing power available today. It is known that paper is a viscoelastic, nearly plastic material, and using this material model inside a fluid-structure interaction problem with fracture is too complex for our purpose. From Fig. 1.1, one can get an insight on the size of paper machines.

Controlling and analyzing paper machines and similar systems and increasing their productivity are goals, which may be approached by modelling. The most often used models for systems involving moving materials have been travelling flexible strings, membranes, beams, and plates. One of the key parameters in safe runnability of paper machines is the web velocity. Using small-displacement theory, which linearizes the web behaviour around the trivial equilibrium, we may find such critical conditions and a critical value for the web velocity, after which the web vibrations grow without bound.

To study the stability of an axially moving web in detail, we need to include in our model different possible disturbances, such as inhomogeneities in web tension. This is important because the occurrence of instability can cause, in particular, tearing the paper web into two parts, and similarly in other applications, e.g. the breakage of transmission cables.

The interaction between the travelling material and the surrounding air has been found to be especially important for applications in paper produc- 


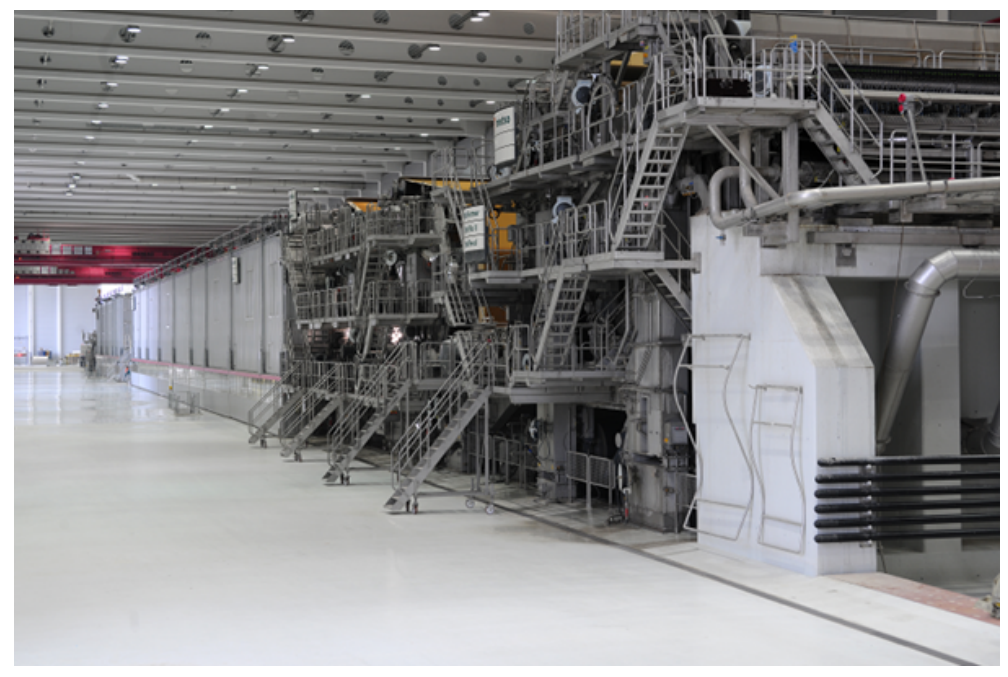

Fig. 1.1 The dimensions of a paper machine. The height of the machine is approximately $8 \mathrm{~m}$, the width $12 \mathrm{~m}$ and the length $120 \mathrm{~m}$. Open draws are mainly hidden inside the drying section, located center left in the photo. (Photo courtesy of Metso Paper)

tion, where the material itself is lightweight, and hence the inertial contribution of the surrounding air to the acceleration of the material is significant. Low bending rigidity of the material also contributes to increasing the relative significance of inertial effects, including those originating in the mechanical interaction with the surrounding fluid.

Including this fluid-structure interaction into the model forms a coupled, multiphysical model. It is not sufficient to consider only the dynamics of the travelling material, but the behaviour of the surrounding airflow must be analyzed, too, and its effects on the material fed back into the structural part of the model. The coupling changes the dynamics, which, in turn, affects the surrounding flow.

If the moving material loses stability, the most likely result (in the case of brittle or quasi-brittle materials such as paper) is that it will break into two parts, interrupting the production process. Such interruptions in paper factories are costly, because when a break occurs, no production takes place, but energy is wasted on running an empty machine. Hence, avoiding web breaks is an important practical question in the design of paper machines. This design, in turn, must take into account the limits created by the fundamental physics of the situation. Thus, fundamental stability limits must be analyzed.

The rest of this book is divided as follows. In Chap. 2 the linear models of travelling strings, panels, and plates are introduced. The formation of the in-plane tension fields, which affect the out-of-plane behaviour, is briefly discussed. 


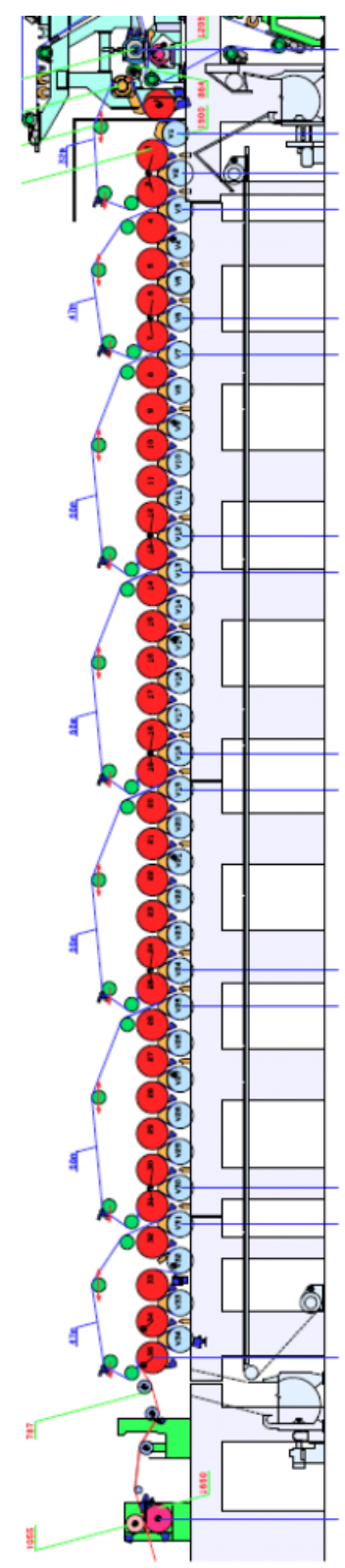

Fig. 1.2 A schematic picture of a paper machine. (Courtesy of Metso Paper) 
Chap. 3 begins with a look into the history of stability investigations, concentrating on moving materials and especially on the extensive studies performed in this field during the last century. After the historical look, Bolotin's linear stability analysis is introduced. Finally, in the main part of the chapter, dynamic and static stability analyses are applied to moving membranes and plates. Critical velocities and divergence shapes are determined analytically.

The model is extended in Chap. 4, where a linearly skewed tension profile at the rollers is accounted for. It is demonstrated for the moving isotropic plate, that a skewed tension profile affects both the critical velocity and the divergence shape.

In Chap. 5, stability and dynamic behaviour of axially moving viscoelastic panels are discussed, applying the classical modal analysis. A model of a thin panel combined with Kelvin-Voigt viscoelasticity is used. In the viscoelastic constitutive relations, the material derivative is used instead of the partial time derivative. For the partial differential equation describing the out-ofplane displacement of the panel, which is of fifth order in space, derivation for the fifth boundary condition is given. In the numerical examples, the behaviour of the eigenfrequencies of the moving viscoelastic panel is illustrated, with respect to the panel velocity, and also the eigenfunctions corresponding to the critical transport velocities are represented. From the numerical examples, it can be observed that the instability of the viscoelastic panel vanishes if the viscosity coefficient is sufficiently high.

Chap. 6 covers the fluid-structure interaction of a moving web with twodimensional potential flow, representing the flow of the surrounding air. Using an exact, analytical functional solution for the pressure difference across the web, the fluid-structure interaction model is reduced to a single integrodifferential equation, which is then solved numerically. This approach simplifies the analysis considerably. Considering that added-mass models are classical in this application area, also an added-mass approximation of the functional solution is derived. However, this is an added bonus; the main analysis is based on the original model, using the exact solution of the pressure difference with no added-mass approximation.

Chap. 7 deals with axially moving plates subject to fracture. A model of a thin elastic plate made of brittle material and having initial cracks is used to describe the behaviour of the plate under constant tension, non-homogeneous tension and cyclic tension. As opposed to the other parts of the book, where we consider one span of the plate or panel, in the case of cyclic tension, we assume the plate to undergo a system of many consecutive spans. We present safe parameter ranges of transport velocities and in-plane tensions accounting for constraints arising from both stability and fracture analyses.

The final chapter, Chap. 8, is continuation for the topics discussed in Chap. 7. In Chap. 8, we discuss some runnability optimization problems, where the physical constraints arising from both stability and fracture analyses are considered. Taking into consideration possible failures arising from a stability loss or material fracture, the chapter deals with safe conditions and process effec- 
tiveness of systems of moving materials. Chap. 8 is divided into three sections. The first section presents the constraints for the plate velocity and for the safe number of cycles and an objective function for process effectiveness. In the second section, we seek optimal in-plane tension that maximizes a performance vector function consisting of the number of cycles before fracture, the critical velocity and process effectiveness. The final section discusses a probabilistic approach for axially moving cracked elastic plates with uncertainties. The length of an initial crack at the plate edge and the magnitude of homogeneous in-plane tension are taken as random variables. Analytical expressions are formulated, and used for computing the optimal tension and the corresponding optimal velocity numerically.

\section{References}

Karlsson M (ed) (2000) Papermaking Science and Technology Vol. 9: Papermaking Part 2: Drying. Fapet Oy, Helsinki, Finland, ISBN 952-5216-09-8

Kenny J (2006) Top 10 global paper companies: 'shrink to fit industry' grapples with overcapacity. URL http://www.thefreelibrary.com/Top 10 global paper companies: 'shrink to fit industry' grapples with...-a0148008393, retrieved April 30, 2013

PricewaterhouseCoopers LLP (2012) Global forest, paper \& packaging industry survey, 2012 edition - survey of 2011 results. URL http://www.pwc.com/en_GX/gx/forest-paper-packaging/assets/pwcglobal-annual-forest-paper-packaging-industry-survey-2012.pdf, retrieved April 30, 2013 



\title{
Chapter 2 Travelling strings, beams, panels, membranes and plates
}

\begin{abstract}
In this chapter, we will introduce in a general manner some of the most common models for axially travelling materials, which will be used in the rest of the book. We will introduce the linear models of travelling strings, panels, and plates. It will be assumed that the material is thin, i.e. its planar dimensions are much larger than its thickness. We will work in the small displacement regime, that is, with linear models approximating the behaviour of the system near the trivial equilibrium. As is well known in the theory of elasticity, this approximation allows for a decoupling of the in-plane and outof-plane components in the dynamics of the system.

We will concentrate on small out-of-plane (transverse) vibrations of the material only, as this is the most relevant aspect of the physics from the viewpoint of dynamical stability, which will be the focus of later chapters. We will look at both one-dimensional and two-dimensional models, and consider variants with and without bending rigidity. The in-plane tension fields, which affect the out-of-plane behaviour, will be considered at the end of the chapter.
\end{abstract}

\subsection{Out-of-plane vibrations}

In the following, we will introduce the four most common linear models for small out-of-plane vibrations of a travelling thin elastic material. These are the travelling string, panel, membrane and plate. The string and the panel are simple one-dimensional models, while the membrane and plate models are two-dimensional, accounting for variations in the displacement along both inplane axes. The panel and the plate resist bending, while the string and the membrane can support only tensile loads. For the membrane and plate, both isotropic and orthotropic variants will be considered. In later chapters, we will examine dynamical stability predictions from these models. 
For the plate model, in this book we focus on rectangular plates with SFSF boundary conditions, where two opposite edges are simply supported (S), and the other two edges are free of tractions (F). For the panel model, we will use the simply supported boundary conditions. For an analysis of the travelling plate in the case of SSSS boundary conditions, i.e., simply supported on all sides, see Luo and Hamidzadeh (2004) and Marynowski (2008).

\subsubsection{Travelling strings}

The simplest way to model a moving material experiencing out-of-plane vibrations is the equation of the travelling string (also known as the threadline equation). The one-dimensional string representation has been used in many fundamental studies and it provides a basis for more advanced analysis. Moreover, it turns out that the behaviour predicted by two-dimensional models of moving materials, when the strip of material is long and narrow, reduces to the corresponding one-dimensional case.

Let us consider an ideal string, which moves axially at a constant transport velocity $V_{0}$ (Fig. 2.1). The transverse (out-of-plane) displacement is described by the function $w=w(x, t)$. By considering the standard wave equation in the co-moving coordinates, and using transformations from Lagrange (material) derivatives to Euler derivatives of transverse displacements,

$$
\frac{\mathrm{d}^{2} w}{\mathrm{~d} t^{2}}=\left(\frac{\partial}{\partial t}+V_{0} \frac{\partial}{\partial x}\right)\left(\frac{\partial w}{\partial t}+V_{0} \frac{\partial w}{\partial x}\right)=\frac{\partial^{2} w}{\partial t^{2}}+2 V_{0} \frac{\partial^{2} w}{\partial x \partial t}+V_{0}^{2} \frac{\partial^{2} w}{\partial x^{2}},
$$

we can write the well-known equation for small displacements of an axially travelling string, describing the motion as it is seen in the Eulerian frame of reference (laboratory coordinates). See e.g. the classical articles by Skutch (1897), Archibald and Emslie (1958) and Swope and Ames (1963). Note that the last form of (2.1) assumes that the axial velocity $V_{0}$ is constant.

The travelling string equation is

$$
m \frac{\partial^{2} w}{\partial t^{2}}+2 m V_{0} \frac{\partial^{2} w}{\partial x \partial t}+\left(m V_{0}^{2}-T\right) \frac{\partial^{2} w}{\partial x^{2}}=0,
$$

where $T$ is the axial tension, having the unit of force, and $m$ is the mass per unit length. In (2.2), the first three terms come from the second material derivative, and the term $T \partial^{2} w / \partial x^{2}$ represents the restoring force of the (vertical projection of the) axial tension.

The customary boundary conditions of the moving string are zero displacement at the both ends of the string (i.e. zero Dirichlet). This describes the situation where a string travels through two pinholes, which are fixed to zero height:

$$
w(-\ell, t)=w(\ell, t)=0 .
$$


A schematic drawing of the setup is presented in Fig. 2.1.

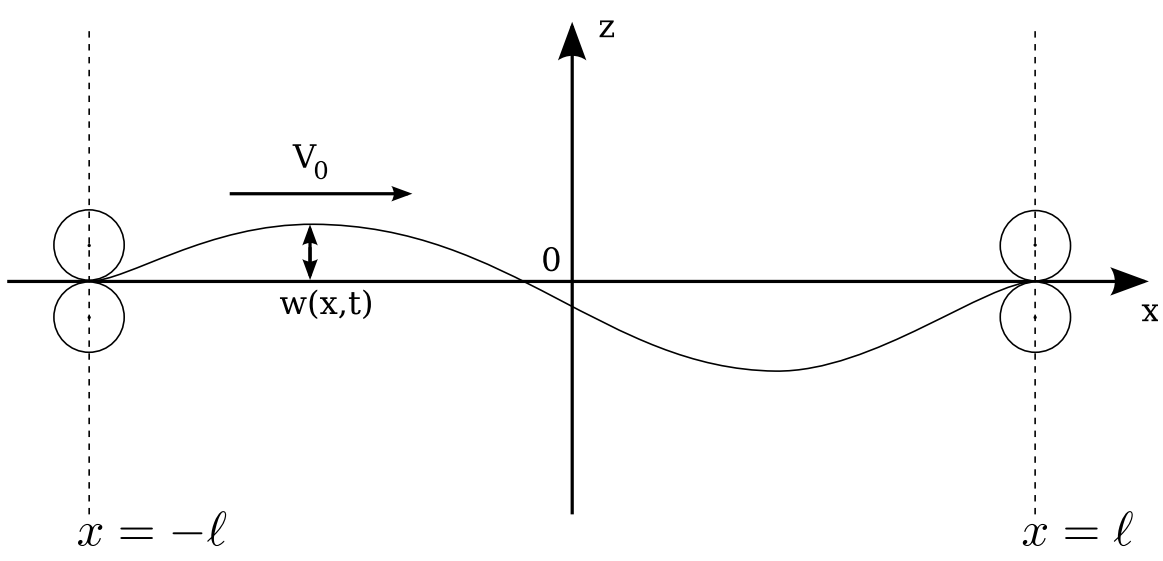

Fig. 2.1 Axially moving ideal string, travelling through two pinholes. The pinholes restrict the displacement at the boundaries to $w=0$. The roller symbols indicate the presence of axial motion. The rollers do not otherwise affect the motion of the string.

An analytical free-vibration solution of the problem (2.2), (2.3) was first reported by Swope and Ames (1963). The complex-valued solution, for nondimensional $x \in(0,1)$ is

$$
w(x, t)=A_{0} \exp \left(\mp i \frac{k \pi \ell}{C \tau}\left[\left(V_{0}^{2}-C^{2}\right) \frac{\tau^{2}}{\ell^{2}} t-V_{0} \frac{\tau}{\ell} x\right]\right) \sin (k \pi x),
$$

where $A_{0}$ is an arbitrary amplitude, $k=1,2,3, \ldots$ is the mode number, $C=\sqrt{T / m}, i=\sqrt{-1}$, and $\tau$ is an arbitrary scaling factor for nondimensionalization of the time coordinate. For a physically meaningful scaling, one can choose e.g. $\tau=\ell / C$. The unit of $\tau$ is $[\tau]=$ s. A real-valued solution is obtained by taking either the real or the imaginary part of (2.4).

The constant $C$, which appears in the solution (2.4), is the propagation speed of a transverse pulse (i.e. the group velocity of waves) in a stationary string. It should be noted that in the moving string, there are two different propagation speeds, $C+V_{0}$ and $C-V_{0}$. Waves moving in the direction of the axial motion of the string propagate at the speed $C+V_{0}$, while those travelling in the opposite direction propagate at the speed $C-V_{0}$. This is a direct consequence of the axial motion. To see this, consider that in the co-moving coordinates, there is no axial motion. Hence, in that frame of reference, waves must propagate at the speed $C$ in both directions. Transforming to the laboratory coordinates leads to the mentioned conclusion.

A systematic derivation of the solution (2.4) is also given in Jeronen (2011), with an extension into the case where first-order damping terms and a linear reaction term (elastic foundation of the classical Winkler type) are added to the model. 


\subsubsection{Travelling panels}

The logical continuation onward from the string model is the travelling panel, for which nonzero bending rigidity is allowed. In this context, the term panel refers to a thin sheet, for which it is assumed that there is no variation in the displacement profile in the width direction (see, e.g., Bisplinghoff and Ashley 1962). It shares mathematical similarities with the classical Euler-Bernoulli beam model, although the physical interpretations are different.

The panel model can be seen as a special case of the plate model, describing the bending of a plate to a cylindrical surface. It follows from the plate model in the limit $\partial / \partial y \rightarrow 0$. In the particular variety discussed here, we consider the dynamic equilibrium for a travelling plate, in the case where the external axial loading in the mid-plane of the plate is significant.

The equation of small transverse vibrations of the travelling panel is

$$
m \frac{\partial^{2} w}{\partial t^{2}}+2 m V_{0} \frac{\partial^{2} w}{\partial x \partial t}+\left(m V_{0}^{2}-T\right) \frac{\partial^{2} w}{\partial x^{2}}+D \frac{\partial^{4} w}{\partial x^{4}}=0
$$

See the setup in Fig. 2.2. Compared to the string case, the dimensions of some of the coefficients are now different. In (2.5), $m$ is the mass of the panel per unit area, and the axial tension $T$ has the dimension of force per unit length. Each term in (2.5) represents a pressure, i.e., force per unit area.

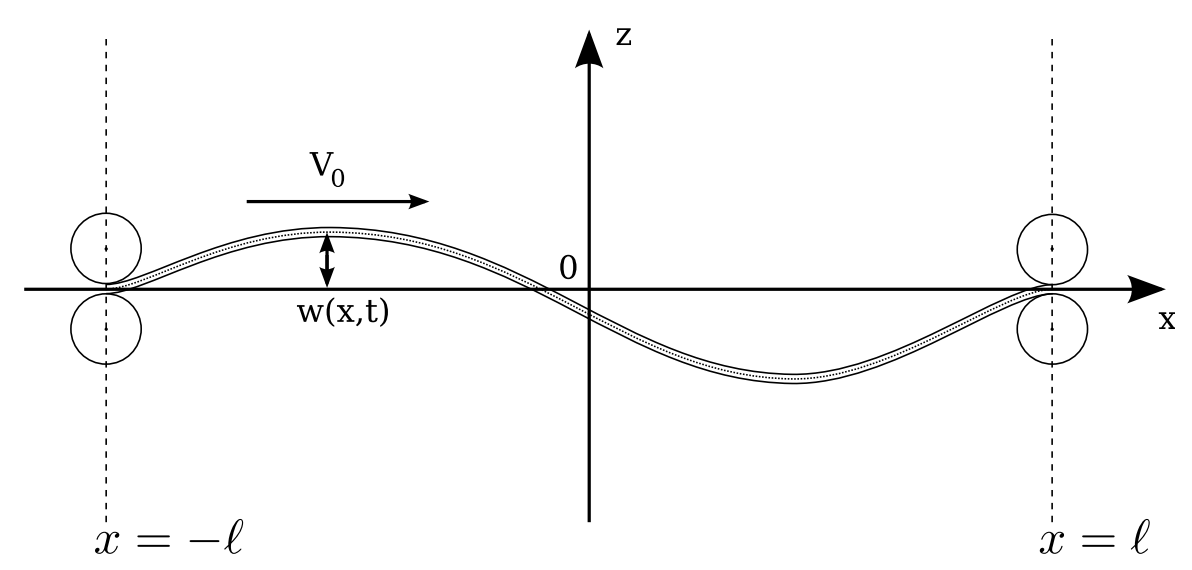

Fig. 2.2 Axially moving panel. The finite thickness depicts bending rigidity. The roller symbols represent simple supports, with presence of axial motion. The function $w(x, t)$ is the transverse displacement of the midplane.

A new term $D \partial^{4} w / \partial x^{4}$ has been introduced, when compared to the string equation (2.2). This term represents the reaction force arising from bending resistance. The quantity $D$ is the bending rigidity from plate theory (Timoshenko and Woinowsky-Krieger 1959, p. 5; also known as cylindrical rigidity): 


$$
D=\frac{E h^{3}}{12\left(1-\nu^{2}\right)},
$$

where $E$ is the Young modulus, $h$ the thickness of the panel, and $\nu$ the Poisson ratio.

The form of (2.5) also applies to a travelling beam. In beam theory, instead of the bending rigidity $D$, the flexural stiffness $E I$ is used, where $E$ is the Young's modulus, and $I$ is the second moment of inertia of the beam crosssection. For the beam, $m$ is taken as mass per unit length, and $T$ has the dimension of force. For the case of the beam, the units of these coefficients agree with those in the string equation.

Equation of transverse panel vibrations (2.5) is of the fourth order in $x$, so four boundary conditions are needed in total. The simply supported (also known as pinned, hinged) boundary conditions are

$$
\begin{aligned}
w(-\ell, t) & =w(\ell, t)=0, \\
\frac{\partial^{2} w}{\partial x^{2}}(-\ell, t) & =\frac{\partial^{2} w}{\partial x^{2}}(\ell, t)=0 .
\end{aligned}
$$

The condition (2.8) arises by requiring that the bending moment at the boundary points is zero. The simply supported conditions represent a panel (or beam), which is free to rotate (but supported) at its endpoints. See Fig. 2.3 , left.

The clamped (or built-in, abbreviation $C$ ) boundary conditions are

$$
\begin{gathered}
w(-\ell, t)=w(\ell, t)=0, \\
\frac{\partial w}{\partial x}(-\ell, t)=\frac{\partial w}{\partial x}(\ell, t)=0 .
\end{gathered}
$$

The condition on $\partial w / \partial x$ ensures that the panel leaves each clamped end perfectly horizontally. The physical situation being modelled by the clamped boundary conditions is usually depicted as having some of the panel (or beam) at its ends extending into a rigid wall. See Fig. 2.3, right.

\subsubsection{Travelling membranes and plates}

In the previous sections, we briefly introduced the string and panel models for travelling continua. These models neglect variation of out-of-plane displacements in the width direction, i.e. in the in-plane direction perpendicular to the axial movement of the material. To obtain a more realistic model of a system where the travelling material is wide, one can use the differential equation for small transverse vibrations of a moving plate.

Consider a rectangular part $\Omega$ of a moving plate in the cartesian coordinate system. Let us define the part $\Omega$ as occupying the region 


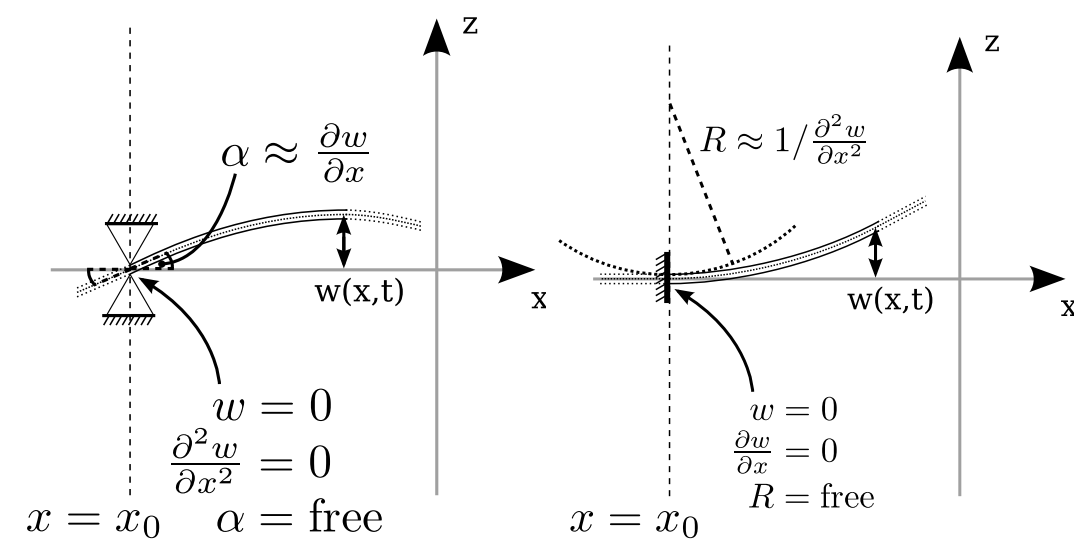

Fig. 2.3 Standard boundary condition types for the panel, illustrated for a stationary panel. Left: simply supported. Right: clamped.

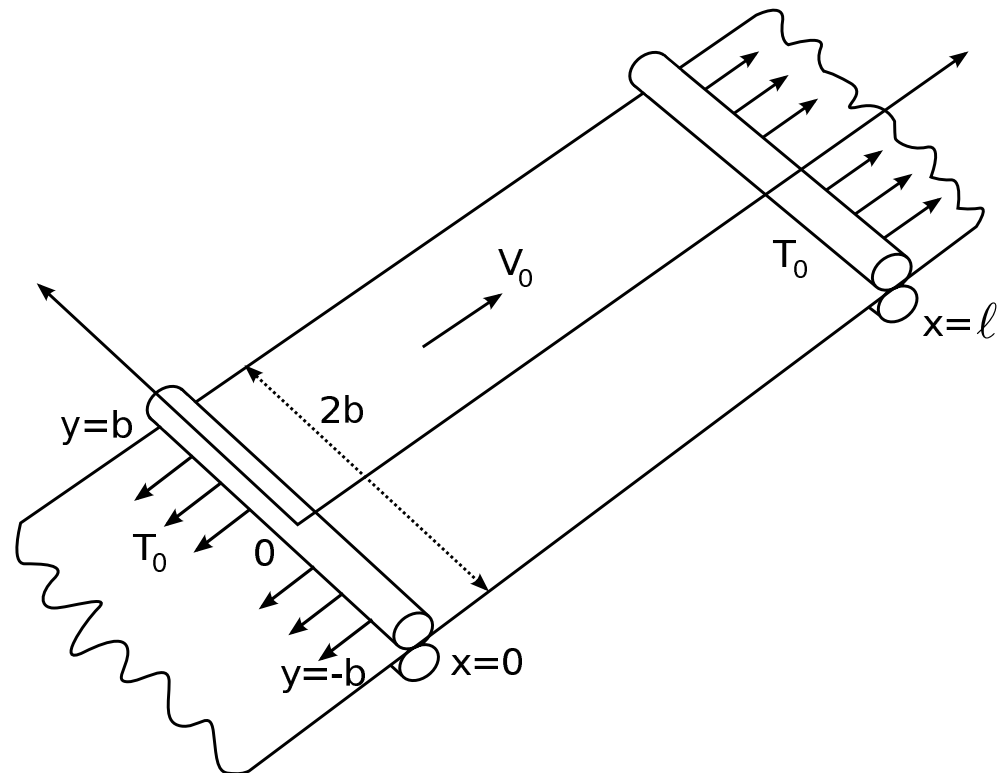

Fig. 2.4 Axially moving plate. The roller symbols represent simple supports, with presence of axial motion. The other two plate edges are assumed to be free of tractions. 


$$
\Omega \equiv\left\{(x, y) \in \mathbb{R}^{2} \mid 0<x<\ell,-b<y<b\right\},
$$

where $b$ and $\ell$ are prescribed geometrical parameters. Figure 2.4 presents the setup.

One can represent the equation for small transverse vibrations of the moving plate in the following form:

$$
m \frac{\mathrm{d}^{2} w}{\mathrm{~d} t^{2}}=\mathcal{L}^{\mathrm{M}}(w)-\mathcal{L}^{\mathrm{B}}(w) .
$$

Here $m$ is the mass per unit area of the plate. For constant axial velocity $V_{0}$, the total acceleration on the left-hand side of (2.12) is expressed as

$$
\frac{\mathrm{d}^{2} w}{\mathrm{~d} t^{2}}=\frac{\mathrm{d}}{\mathrm{d} t}\left(\frac{\partial w}{\partial t}+V_{0} \frac{\partial w}{\partial x}\right)=\frac{\partial^{2} w}{\partial t^{2}}+2 V_{0} \frac{\partial^{2} w}{\partial x \partial t}+V_{0}^{2} \frac{\partial^{2} w}{\partial x^{2}} .
$$

The right-hand side in (2.13) contains three terms, representing local acceleration, Coriolis acceleration, and centripetal acceleration, respectively. The membrane operator $\mathcal{L}^{\mathrm{M}}$ on the right-hand side of (2.12) is

$$
\mathcal{L}^{\mathrm{M}}(w)=T_{x x} \frac{\partial^{2} w}{\partial x^{2}}+2 T_{x y} \frac{\partial^{2} w}{\partial x \partial y}+T_{y y} \frac{\partial^{2} w}{\partial y^{2}} .
$$

The coefficients $T_{x x}, T_{x y}$, and $T_{y y}$ of the linear operator $\mathcal{L}^{\mathrm{M}}$ are related to the corresponding in-plane stresses $\sigma_{x x}, \sigma_{x y}$ and $\sigma_{y y}$ by the expressions

$$
T_{i j}=h \sigma_{i j},
$$

where $h$ is the thickness of the plate, assumed constant. The linear bending operator $\mathcal{L}^{\mathrm{B}}$ is given by the expression

$$
\mathcal{L}^{\mathrm{B}}(w)=D \triangle^{2} w=D\left(\frac{\partial^{4} w}{\partial x^{4}}+2 \frac{\partial^{4} w}{\partial x^{2} \partial y^{2}}+\frac{\partial^{4} w}{\partial y^{4}}\right),
$$

in the case of an isotropic elastic plate. Here, $\triangle^{2}$ is the biharmonic operator and $D$ is the bending rigidity of an isotropic plate (or panel) defined in (2.6). When we consider the case of an orthotropic plate, the operator $\mathcal{L}^{B}$ depends on three constants, being written as

$$
\mathcal{L}^{\mathrm{B}}(w)=D_{1} \frac{\partial^{4} w}{\partial x^{4}}+2 D_{3} \frac{\partial^{4} w}{\partial x^{2} \partial y^{2}}+D_{2} \frac{\partial^{4} w}{\partial y^{4}} .
$$

In the case of a membrane, the bending rigidities are neglected and the entire operator $\mathcal{L}^{\mathrm{B}}$ is omitted $\left(\mathcal{L}^{\mathrm{B}} \equiv 0\right)$. In the case of a plate, we have the following expressions (see, e.g., Timoshenko and Woinowsky-Krieger 1959) for the bending rigidities in (2.17): 


$$
D_{1}=\frac{h^{3}}{12} C_{11}, \quad D_{2}=\frac{h^{3}}{12} C_{22}, \quad D_{3}=\frac{h^{3}}{12}\left(C_{12}+2 C_{66}\right),
$$

where $C_{i j}$ are the elastic moduli. These can be expressed in terms of the Young moduli $E_{1}, E_{2}$ and Poisson ratios $\nu_{12}, \nu_{21}$ as (see, e.g., Kikuchi 1986),

$$
\begin{gathered}
C_{11}=\frac{E_{1}}{1-\nu_{12} \nu_{21}}, \quad C_{22}=\frac{E_{2}}{1-\nu_{12} \nu_{21}}, \\
C_{12}=\frac{\nu_{21} E_{1}}{1-\nu_{12} \nu_{21}}=\frac{\nu_{12} E_{2}}{1-\nu_{12} \nu_{21}}=C_{21}, \\
C_{66}=G_{12} .
\end{gathered}
$$

In (2.19), $E_{1}$ is the Young modulus in the $x$ direction, and $\nu_{12}$ is the Poisson ratio in the $x y$ plane when the stretching is applied in the $x$ direction. Respectively, $E_{2}$ is the Young modulus in the $y$ direction, and $\nu_{21}$ is the Poisson ratio in the $x y$ plane when the stretching is applied in $y$ direction. $G_{12}$ is the shear modulus in the $x y$ plane.

We assume that the deflection function $w$ and its partial derivatives are small, and that they satisfy the boundary conditions. In the case of an orthotropic plate, the boundary conditions read

$$
\begin{array}{rlr}
(w)_{x=0, \ell}=0, \quad\left(\frac{\partial^{2} w}{\partial x^{2}}\right)_{x=0, \ell}=0, & -b \leq y \leq b, \\
\left(\frac{\partial^{2} w}{\partial y^{2}}+\beta_{1} \frac{\partial^{2} w}{\partial x^{2}}\right)_{y= \pm b}=0, & 0 \leq x \leq \ell, \\
\left(\frac{\partial^{3} w}{\partial y^{3}}+\beta_{2} \frac{\partial^{3} w}{\partial x^{2} \partial y}\right)_{y= \pm b}=0, & 0 \leq x \leq \ell,
\end{array}
$$

representing two opposite edges simply supported and the other two edges free of tractions.

The mechanical parameters $\beta_{1}$ and $\beta_{2}$ in the free-edge boundary conditions are defined as

$$
\begin{gathered}
\beta_{1}=\nu_{12}, \\
\beta_{2}=\nu_{12}+\frac{4 G_{12}}{E_{2}}\left(1-\nu_{12} \nu_{21}\right) .
\end{gathered}
$$

For the isotropic plate, the mechanical parameters $\beta_{1}$ and $\beta_{2}$ simplify to

$$
\beta_{1}=\nu \quad \text { and } \quad \beta_{2}=2-\nu .
$$

This can be seen by setting $\nu_{12}=\nu_{21}=\nu, E_{1}=E_{2}=E, G_{12}=G$, and using the isotropic shear modulus relation $G=E /(2(1+\nu))$. Then, factoring $1-\nu_{12} \nu_{21}=1-\nu^{2}=(1+\nu)(1-\nu)$ and simplifying reduces $(2.23)$ into $(2.24)$. 
In the $x$ direction, we use the simply supported boundary conditions (2.20) for the sake of simplicity. From a physical point of view, it is an important and nontrivial question which type boundary conditions to select for the rollers. The simply supported conditions, however, are a standard choice.

Using the geometric average approximation $G_{\mathrm{H}}$ for the shear modulus $G_{12}$,

$$
G_{\mathrm{H}} \equiv \frac{\sqrt{E_{1} E_{2}}}{2\left(1+\sqrt{\nu_{12} \nu_{21}}\right)}
$$

the equations for the (classical, stationary) orthotropic plate reduce to those of an isotropic plate. The geometric average shear modulus in (2.25) was introduced by Huber (1923), generalizing the shear modulus for orthotropic materials.

It is easy to show that the reduction property of $G_{\mathrm{H}}$ remains valid for the time-dependent travelling plate problem as well. Because in the transformations producing this reduction, coordinate scaling is only required in the $y$ direction (see Timoshenko and Woinowsky-Krieger 1959, chap. 11), the Coriolis term $\partial^{2} w / \partial x \partial t$ generated by the axial motion of the plate does not alter the approach.

In this book, unless otherwise noted, we will assume that $G_{12}$ is an independent material parameter. This is motivated by the application point of view, where the shear modulus generally differs from its geometric average especially for paper materials.

In the case of a membrane, the boundary condition at the rollers reads

$$
(w)_{x=0, \ell}=0, \quad-b \leq y \leq b .
$$

On the free edges, classical membrane theory asserts (see e.g. Sagan 1961 and Weinstock 2008)

$$
\left(\frac{\partial w}{\partial y}\right)_{y= \pm b}=0, \quad 0 \leq x \leq \ell
$$

but this is not the only possible boundary condition. Shin et al (2005) have used for zero traction a different condition, which does not contain the transverse displacement $w$. We will see below that in our case for a membrane, both choices of the boundary condition on the free edges are possible.

\subsection{In-plane tensions}

The stability of moving materials is sensitive to the in-plane tensions. The higher the tension, the larger the region of transport velocities at which the system remains stable. Next we describe the stationary representation of inplane forces, i.e. we assume that the in-plane tensions do not depend on time $t$. The in-plane tensions $T_{x x}, T_{x y}$ and $T_{y y}$ are assumed to satisfy the 
equilibrium equations (see, e.g., Timoshenko and Goodier 1951)

$$
\frac{\partial T_{x x}}{\partial x}+\frac{\partial T_{x y}}{\partial y}=0, \quad \frac{\partial T_{x y}}{\partial x}+\frac{\partial T_{y y}}{\partial y}=0 .
$$

The in-plane tensions $T_{x x}, T_{x y}$ and $T_{y y}$ are related to the corresponding stress tensor components $\sigma_{x x}, \sigma_{x y}$ and $\sigma_{y y}$ by the relation (2.15), i.e.,

$$
T_{i j}=h \sigma_{i j} .
$$

For a rectangular band of material stretched at opposite ends with no shear, and free of tractions at the two other edges, the boundary conditions are

$$
\begin{gathered}
T_{x x}=k(y), \quad T_{x y}=0 \quad \text { at } x=0, \ell, \quad-b \leq y \leq b, \\
T_{y y}=0, \quad T_{x y}=0 \quad \text { at } y= \pm b, \quad 0 \leq x \leq \ell .
\end{gathered}
$$

Let us begin with a homogeneous tension field, where $k(y)$ degenerates to a constant, $T_{0}$. Taking into account the behavioral equation of the plane theory of elasticity and the boundary conditions (2.29) and (2.30), we have for the band considered the tension field

$$
T_{x x}=T_{0}, \quad T_{y y}=T_{x y}=0, \quad(x, y) \in \Omega,
$$

where

$$
\Omega \equiv\left\{(x, y) \in \mathbb{R}^{2} \mid 0<x<\ell,-b<y<b\right\} .
$$

For an orthotropic band, the in-plane displacements $u$, and $v$, oriented respectively along the axes $x$ and $y$, are related to the stresses by means of the generalized Hooke's law:

$$
\begin{aligned}
\sigma_{x x} & =C_{11} \frac{\partial u}{\partial x}+C_{12} \frac{\partial v}{\partial y}, \\
\sigma_{x y} & =C_{66}\left(\frac{\partial u}{\partial y}+\frac{\partial v}{\partial x}\right) \text { and } \\
\sigma_{y y} & =C_{21} \frac{\partial u}{\partial x}+C_{22} \frac{\partial v}{\partial y},
\end{aligned}
$$

where $C_{i j}$ are the elastic moduli, see (2.19), and we have used the linear strain-displacement relations

$$
\begin{aligned}
\varepsilon_{x x} & =\frac{\partial u}{\partial x}, \\
\gamma_{x y} & =\frac{\partial u}{\partial y}+\frac{\partial v}{\partial x}, \\
\varepsilon_{y y} & =\frac{\partial v}{\partial y} .
\end{aligned}
$$


In the following, we will use the compatibility relation

$$
E_{1} \nu_{21}=E_{2} \nu_{12}
$$

which is implied by the symmetricity of the elastic moduli, i.e. $C_{12}=C_{21}$.

It is possible to show that if instead of a prescribed tension $T_{0}$, we have a prescribed displacement $u_{0}$ at $x=\ell$, the generated tension field has the same form as the form given by (2.31).

Using (2.15), (2.28) and (2.32-2.34), we obtain the stress equilibrium equations in terms of the in-plane displacements $u$ and $v$ :

$$
\begin{aligned}
& C_{11} \frac{\partial^{2} u}{\partial x^{2}}+\left(C_{12}+C_{66}\right) \frac{\partial^{2} v}{\partial x \partial y}+C_{66} \frac{\partial^{2} u}{\partial y^{2}}=0 \\
& C_{22} \frac{\partial^{2} v}{\partial y^{2}}+\left(C_{12}+C_{66}\right) \frac{\partial^{2} u}{\partial x \partial y}+C_{66} \frac{\partial^{2} v}{\partial x^{2}}=0 .
\end{aligned}
$$

The boundary conditions in terms of $u$ and $v$ are

$$
\begin{gathered}
\frac{\partial u}{\partial y}+\frac{\partial v}{\partial x}=0 \quad \text { at } x=0, \ell, \quad-b \leq y \leq b, \\
u=0 \quad \text { at } x=0, \quad-b \leq y \leq b, \\
u=u_{0} \quad \text { at } x=\ell, \quad-b \leq y \leq b,
\end{gathered}
$$

and (compare with (2.30))

$$
\begin{gathered}
C_{12} \frac{\partial u}{\partial x}+C_{22} \frac{\partial v}{\partial y}=0 \quad \text { at } y= \pm b, \quad 0 \leq x \leq \ell, \\
\frac{\partial u}{\partial y}+\frac{\partial v}{\partial x}=0 \quad \text { at } y= \pm b, \quad 0 \leq x \leq \ell .
\end{gathered}
$$

From the equilibrium (2.37) and the boundary conditions (2.38-2.39), the displacement field in the domain $\Omega$ is described as

$$
u(x, y)=\frac{u_{0}}{\ell} x, \quad v(x, y)=-\frac{C_{12}}{C_{22}} \frac{u_{0}}{\ell} y, \quad(x, y) \in \Omega .
$$

Inserting these into (2.32-2.34) and using (2.15), we see that the tension field has the form given by (2.31) with the constant value $T_{x x}=T_{0}$, where

$$
T_{0}=h \frac{u_{0}}{\ell}\left(C_{11}-\frac{C_{12}^{2}}{C_{22}}\right)=h \frac{u_{0}}{\ell} E_{1} .
$$

From (2.19) and (2.36), the last form in (2.40) easily follows. We see that the only material parameter that affects the homogeneous tension field generated 
by the prescribed displacement is the Young modulus in the longitudinal direction. Compare (2.40) with

$$
T_{0}=h \frac{u_{0}}{\ell} E
$$

for an isotropic material.

For non-homogeneous tension in an isotropic sheet of material, one classical way to find compatible tensions $T_{x x}, T_{y y}$ and $T_{x y}$ is via the help of an Airy stress function $\Upsilon$. Let us do this for an orthotropic sheet. As in the classical isotropic case, we define the tensions in terms of second derivatives of the Airy stress function as

$$
\begin{aligned}
& T_{x x}=h \sigma_{x x}=\frac{\partial^{2} \Upsilon}{\partial y^{2}}, \\
& T_{y y}=h \sigma_{y y}=\frac{\partial^{2} \Upsilon}{\partial x^{2}}, \\
& T_{x y}=h \sigma_{x y}=-\frac{\partial^{2} \Upsilon}{\partial x \partial y} .
\end{aligned}
$$

Consider now the inverse relation of the generalized Hooke's law (2.322.34). We can express it as

$$
\begin{aligned}
& \varepsilon_{x x}=\frac{1}{E_{1}} \sigma_{x x}-\frac{\nu_{21}}{E_{2}} \sigma_{y y}, \\
& \gamma_{x y}=\frac{1}{G_{12}} \sigma_{x y}, \\
& \varepsilon_{y y}=\frac{1}{E_{2}} \sigma_{y y}-\frac{\nu_{12}}{E_{1}} \sigma_{x x} .
\end{aligned}
$$

From the linear strain-displacement relations (2.35), it follows that the strain variables satisfy the strain compatibility equation (see, e.g., Timoshenko and Goodier 1951)

$$
\frac{\partial^{2} \varepsilon_{x x}}{\partial y^{2}}+\frac{\partial^{2} \varepsilon_{y y}}{\partial x^{2}}-\frac{\partial^{2} \gamma_{x y}}{\partial x \partial y}=0 .
$$

By inserting (2.42) and (2.43) into (2.44) (and noting that $T_{i j}=h \sigma_{i j}$ ), and using (2.36), we see that for an orthotropic material, the Airy stress function $\Upsilon$ must satisfy the equation

$$
\frac{\partial^{4} \Upsilon}{\partial x^{4}}+\left(\frac{E_{2}}{G_{12}}-2 \nu_{21}\right) \frac{\partial^{4} \Upsilon}{\partial x^{2} \partial y^{2}}+\frac{E_{2}}{E_{1}} \frac{\partial^{4} \Upsilon}{\partial y^{4}}=0 .
$$

In the isotropic case, by setting $E_{1}=E_{2}=E, G_{12}=G, \nu_{21}=\nu$, and using the isotropic shear modulus relation $G=E /(2(1+\nu))$, we observe that (2.45) reduces to the well-known biharmonic equation 


$$
\Delta^{2} \Upsilon \equiv \frac{\partial^{4} \Upsilon}{\partial x^{4}}+2 \frac{\partial^{4} \Upsilon}{\partial x^{2} \partial y^{2}}+\frac{\partial^{4} \Upsilon}{\partial y^{4}}=0 \quad \text { (for isotropic material) }
$$

For an axially tensioned rectangular band of material, the boundary conditions satisfied by $\Upsilon$, corresponding to (2.29) and (2.30), are

$$
\begin{gathered}
\left(\frac{\partial^{2} \Upsilon}{\partial y^{2}}\right)_{x=0, \ell}=k(y), \quad\left(\frac{\partial^{2} \Upsilon}{\partial x \partial y}\right)_{x=0, \ell}=0, \quad-b \leq y \leq b, \\
\left(\frac{\partial^{2} \Upsilon}{\partial x^{2}}\right)_{y= \pm b}=0, \quad\left(\frac{\partial^{2} \Upsilon}{\partial x \partial y}\right)_{y= \pm b}=0, \quad 0 \leq x \leq \ell .
\end{gathered}
$$

The tensions expressed via the stress function $\Upsilon$ in (2.42) will satisfy the stress equilibrium equations (2.28) for any function $\Upsilon$ that is sufficiently smooth. The problem (2.45), (2.47-2.48), which must be solved, expresses the condition of compatibility for the tensions.

Later, in Chap. 4, we will concentrate on a linear tension distribution, and use the rigorous solution of the boundary value problem (2.45), (2.47-2.48) corresponding to the case where the tension profile function in $(2.29),(2.47)$ is taken as

$$
k(y)=T_{0}+\alpha y .
$$

Here $\alpha>0$ is a given constant, characterizing the skew of the linear tension profile. We have

$$
\Upsilon(x, y)=T_{0} \frac{y^{2}}{2}+\alpha \frac{y^{3}}{6}+c_{1} x+c_{2} y+c_{0}, \quad(x, y) \in \Omega .
$$

The corresponding tensions will be

$$
T_{x x}(x, y)=T_{0}+\alpha y, \quad T_{x y}(x, y)=0, \quad T_{y y}(x, y)=0,
$$

where $(x, y) \in \Omega$, and $c_{0}, c_{1}$ and $c_{2}$ are arbitrary constants.

\section{References}

Archibald FR, Emslie AG (1958) The vibration of a string having a uniform motion along its length. ASME Journal of Applied Mechanics 25:347-348

Bisplinghoff RL, Ashley H (1962) Principles of Aeroelasticity. Dover Publications, Inc., New York, 2nd edition, 1975

Huber MT (1923) Die Theorie des kreuzweise bewehrten Eisenbetonplatten. Der Bauingenieur 4:354-392

Jeronen J (2011) On the mechanical stability and out-of-plane dynamics of a travelling panel submerged in axially flowing ideal fluid: a study into paper production in mathematical terms. PhD thesis, Depart- 
ment of Mathematical Information Technology, University of Jyväskylä, URL http://julkaisut.jyu.fi/?id=978-951-39-4596-1, Jyväskylä studies in computing 148. ISBN 978-951-39-4595-4 (book), ISBN 978-951-39-4596-1 (PDF)

Kikuchi N (1986) Finite Element Methods in Mechanics. Cambridge University Press, Cambridge, UK, ISBN 0521339723

Luo ACJ, Hamidzadeh HR (2004) Equilibrium and buckling stability for axially traveling plates. Communications in Nonlinear Science and Numerical Simulation 9(3):343-360, URL http://dx.doi.org/10.1016/S1007$5704(02) 00132-6$

Marynowski K (2008) Dynamics of the Axially Moving Orthotropic Web, Lecture Notes in Applied and Computational Mechanics, vol 38. SpringerVerlag, Germany

Sagan H (1961) Boundary and Eigenvalue Problems in Mathematical Physics. John Wiley \& Sons, Inc., slightly corrected reprint by Dover Publications, Inc., 1989.

Shin C, Chung J, Kim W (2005) Dynamic characteristics of the out-of-plane vibration for an axially moving membrane. Journal of Sound and Vibration 286(4-5):1019-1031

Skutch R (1897) Uber die Bewegung eines gespannten Fadens, weicher gezwungen ist durch zwei feste Punkte, mit einer constanten Geschwindigkeit zu gehen, und zwischen denselben in Transversalschwingungen von gerlinger Amplitude versetzt wird. Annalen der Physik und Chemie 61:190-195

Swope RD, Ames WF (1963) Vibrations of a moving threadline. Journal of the Franklin Institute 275:36-55

Timoshenko S, Goodier J (1951) Theory of Elasticity, second edition edn. McGraw-Hill

Timoshenko SP, Woinowsky-Krieger S (1959) Theory of plates and shells, 2nd edn. New York : Tokyo : McGraw-Hill, ISBN 0-07-085820-9

Weinstock R (2008) Calculus of Variations - With Applications to Physics and Engineering. Weinstock Press, ISBN 978-1443728812. Reprint of 1974 edition. 


\title{
Chapter 3 Stability analysis
}

\begin{abstract}
The focus of this chapter is the stability analysis of axially moving materials. There are many similarities with classical stability analysis of structures, like the buckling of beams and plates. However, the axial motion introduces the effects of inertia, bringing out many challenges that are discussed in this chapter. The chapter is divided as follows. In the first section, we will look into the history of stability investigations, concentrating on moving materials and especially on the extensive studies performed in this field during the last century. In the second section, we will introduce linear stability analysis using Bolotin's concept of dynamical stability. Finally, in the last three sections, dynamic and static stability analyses will be applied to moving membranes and plates.
\end{abstract}

\subsection{Historical view of stability investigations}

Stability analysis comes with a long tradition. The steady-state stability of parabolic shapes partially immersed in a homogeneous medium was analyzed in the two-part book On Floating Bodies by Archimedes of Syracuse. The book, originally dating from the 3rd century BCE, can be thought of as the oldest surviving work on stability analysis; its probable application was shipbuilding (Russo 2004).

The present form of static stability analysis, which will be applied in this book, was originally developed by Euler (1766), for a differential equation describing the bending of a beam. The dynamic stability analysis for linear elastic systems, which extends Euler's method, is due to Bolotin (1963). According to Mote and Wickert (1991), the instability behaviour of some axially moving materials is mathematically analogous to the buckling of a compressed column, enabling the use of these techniques. 
In the following, we will limit the scope of our consideration to moving materials. The first investigation in this area was performed by Skutch (1897) being published originally in German. The first English-language paper on the topic was published half a century later by Sack (1954). Both of these studies discussed the vibrations of a travelling string. Interest in the field then arose quickly. In a short study a few years later, by Archibald and Emslie (1958), two ways to derive the travelling string equation were presented. The first analytical solution to the travelling string problem concerned the free vibrations. It was obtained by Swope and Ames (1963), using a coordinate transform approach.

The dynamic and stability considerations discussed here were first reviewed in the article by Mote (1972). Natural frequencies are commonly analyzed together with the stability. The effects of axial motion of the web on its frequency spectrum and eigenfunctions were investigated in the papers by Archibald and Emslie (1958) and by Simpson (1973). It was shown that the natural frequency of each mode decreases when the transport speed increases, and that the travelling string and beam both experience divergence instability at a sufficiently high speed. However, in the case of the string, this result was recently contrasted by Wang et al (2005), who showed using Hamiltonian mechanics that the ideal string remains stable at any speed. Travelling beams have been further analyzed by Parker (1998) in his study on gyroscopic continua, and by Kong and Parker (2004), where an approximate analytical expression was derived for the eigenfrequencies of a moving beam with small flexural stiffness.

Response predictions have been made for particular cases where the excitation assumes special forms, such as harmonic support motion (Miranker 1960) or a constant transverse point force (Chonan 1986). Arbitrary excitations and initial conditions were analyzed with the help of modal analysis and a Green's function method in the article by Wickert and Mote (1990). As a result, the critical speeds for travelling strings and beams were explicitly determined.

The loss of stability was studied with an application of dynamic and static approaches in the article by Wickert (1992). It was shown by means of numerical analysis that in all cases instability occurs when the frequency is zero and the critical velocity coincides with the corresponding velocity obtained from static analysis.

Two-dimensional studies have also been performed from the 1990s onwards. For example, Lin and Mote (1995) studied an axially moving membrane in a $2 \mathrm{D}$ formulation, predicting the equilibrium displacement and stress distributions under transverse loading. In the article by Shin et al (2005), outof-plane vibrations of an axially moving membrane were studied. They also found by numerical analysis that for a membrane with no-friction boundary conditions in the lateral direction along the rollers, the membrane remains dynamically stable until the critical speed, at which static instability occurs, is reached. Lin and Mote (1996) extended their study, predicting a wrinkling 
instability and the corresponding wrinkled shape of a web with small flexural stiffness. Lin (1997) continued the studies of stability.

It was realized early on that the vibration problem for an axially moving continuum is not the conventional one. Because of the longitudinal continuity of the material, the equation of motion for transverse vibration will contain additional terms, representing a Coriolis force and a centripetal force acting on the material. As a consequence, the resonant frequencies will be dependent on the longitudinal velocity of the axially moving continuum, as was noted by Archibald and Emslie (1958), as well as Swope and Ames (1963), Simpson (1973), and Mujumdar and Douglas (1976).

In the 1980s, it was discovered that another important factor affecting the stability of the axially moving continuum, especially if the material itself is lightweight, is the interaction between the moving continuum and the surrounding medium (Pramila 1986). The interaction between the travelling continuum and the surrounding air is known to influence the critical velocity (Pramila 1986; Frondelius et al 2006) and the dynamical response (Kulachenko et al 2007b), possibly also affecting the divergence (buckling) shape. The mentioned studies concentrate on paper making, but the same phenomenon is encountered also in other applications. For example, in a paper by Hosaka and Crandall (1992), the vibrations of an elastic disc rotating above an air film were investigated.

The simplest approach to taking into account the fluid-structure interaction is to assume potential flow; that is, the surrounding air is assumed to be incompressible and inviscid, and the flow is assumed to be irrotational (like in e.g. Niemi and Pramila 1986). Experimental studies and some theoretical estimations (see, e.g., Pramila 1986) indicate that in the case of normal vibration, comparison of experimental and theoretical results shows that predictions based on the potential flow theory are within about $10 \%$ of the measured results. To solve the external hydrodynamic problem, and to find the reaction of the surrounding medium, the finite element method has been used (e.g. Niemi and Pramila 1986).

A closely related problem is the response of stationary material to a surrounding axial flow. It has been noted Païdoussis (2008) that this problem, in turn, is related to the canonical problem of the fluid-conveying pipe. However, the case of material surrounded by axial flow is more complicated than the case of the pipe, due to the nonlocal nature of the aerodynamic reaction. The problems of slender structures in axial flow have been studied extensively, and are summarized in the two-volume book by Païdoussis $(1998,2004)$.

Returning to moving materials, the dynamical properties of moving plates have been studied by Shen et al (1995) and by Shin et al (2005), and the properties of a moving paper web have been studied in the two-part article by Kulachenko et al $(2007 \mathrm{a}, \mathrm{b})$. Critical regimes and other problems of stability analysis have been studied by Wang (2003) and Sygulski (2007).

Results that axially moving beams experience divergence instability at a sufficiently high beam velocity have been obtained also for beams interact- 
ing with external media; see, e.g., Chang and Moretti (1991), and Banichuk et al (2010b, 2011b); Jeronen (2011). The same authors have extended the study in Banichuk et al (2010a, 2011a), for a two-dimensional model of the web, considered as a moving plate under homogeneous tension but without external media. These studies have been further extended in Banichuk et al (2013) and Tuovinen (2011) to the case with a linear non-homogeneous tension distribution (see also Chapter 4).

The mechanical behavior of a paper web under a non-failure condition is adequately described by the model of an elastic orthotropic plate. The rigidity coefficients of the plate model that describe the tension and bending of the paper sheet have been estimated for various types of paper in many publications. See, for example, the articles by Göttsching and Baumgarten (1976), by Thorpe (1981), by Skowronski and Robertson (1985) and by Seo (1999). The deformation properties of a sheet of paper under tensile stress or strain are used in simulation of axial movement of a paper web. In particular, these properties are important for the modeling of the instability of the web.

In a recent article by Hatami et al (2009), the free vibration of a moving orthotropic rectangular plate was studied at sub- and supercritical speeds, and its flutter and divergence instabilities at supercritical speeds. The study is limited to simply supported boundary conditions at all edges. For the solution of equations of orthotropic moving material, many necessary fundamentals can be found in the book by Marynowski (2008).

The free vibrations of stationary orthotropic rectangular plates have been extensively studied. The classical reference work in this area is the book by Gorman (1982). More recently, Biancolini et al (2005) included in their study all combinations of simply supported and clamped boundary conditions on the edges. Xing and Liu (2009) obtained exact solutions for the free vibrations of stationary rectangular orthotropic plates. They considered three combinations of simply supported (S) and clamped (C) boundary conditions: SSCC, SCCC, and CCCC. Kshirsagar and Bhaskar (2008) studied vibrations and buckling of loaded stationary orthotropic plates. They found critical loads of buckling for all combinations of boundary conditions S, C, and F.

Recently, attention has turned toward the material model, which is also an important factor in the stability behaviour of a moving material. Industrial materials often have viscoelastic characteristics (see, e.g., Fung et al 1997), and consequently, viscoelastic moving materials have been recently studied widely. In paper making, wet paper webs are highly viscous, and therefore, viscoelasticity should be taken into account in the models (see, e.g., Alava and Niskanen 2006). Also plasticity is known to occur (see, e.g., Erkkilä et al 2013); however that topic is beyond the scope of the present book.

First studies on transverse vibration of viscoelastic material traveling between two fixed supports were done by Fung et al (1997), using a string model. Extending the work, they studied the material damping effect in Fung et al (1998). 
Several studies on travelling viscoelastic materials, concerning strings and beams, have been performed during the last decade. Chen and Zhao (2005) represented a modified finite difference method to simplify a non-linear model of an axially moving viscoelastic string. They studied the free transverse vibrations of elastic and viscoelastic strings numerically.

Oh et al (2004) and Lee and Oh (2005) studied critical speeds, eigenvalues, and natural modes of axially moving viscoelastic beams using the spectral element model. They analyzed dynamic behavior of axially moving viscoelastic beams using modal analysis, performed a detailed eigenfrequency analysis, and reported that viscoelasticity did not affect the critical velocity of the beam.

Marynowski and Kapitaniak (2002) compared two different internal damping models in modeling of moving viscoelastic (non-linear) beams. For the linearized Kelvin-Voigt model, it was found that the beam exhibits divergent instability at some critical speed. In the case of non-linear BÃCErgers model, the critical speed decreased when the internal damping was increased, and the beam was found to experience the first instability in the form of flutter.

A few studies on transverse vibrations of axially moving viscoelastic plates have also been done. Very recently, Yang et al (2012) studied vibrations, bifurcation and chaos of axially moving viscoelastic plates using finite differences and a non-linear model for transverse displacements. They concentrated on bifurcations and chaos, but also studied the dynamic characteristics of a linearised elastic model with the help of eigenfrequency analysis.

A particular question about whether one should use the material time derivative or the partial time derivative in the viscoelastic constitutive relations for moving materials, has recently been discussed especially in the case of the widely used Kelvin-Voigt material model. Mockensturm and Guo (2005) suggested that the material derivative should be used. They studied non-linear vibrations and dynamic response of axially moving viscoelastic strings, and found significant discrepancy in the frequencies at which nontrivial limit cycles exist, comparing the models with the partial time derivative to those with the material time derivative.

Recently, the material derivative has been used in most of the studies concerning axially moving viscoelastic beams (see e.g. the papers by Chen et al 2008, Chen and Ding 2010, Chen and Wang 2009, and Ding and Chen 2008). Kurki and Lehtinen (2009) suggested, independently, that the material derivative in the constitutive relations should be used in their study concerning the in-plane displacement field of a traveling viscoelastic plate. Some more studies specifically about viscoelastic moving materials will be introduced in Chap. 5 . 


\subsection{Linear stability analysis}

The most straightforward and efficient way to study stability is to use linear stability analysis. It is well-known that the normal vibrations of an elastic linear system are time-harmonic (this is noted by e.g. Xing and Liu 2009). For the stability analysis of all such systems described by partial differential equation models, it is standard to use the time-harmonic trial function

$$
w(x, t)=\exp (s t) W(x),
$$

where $s$ is complex, $W(x)$ is an unknown eigenmode to be determined and $x$ is a scalar or a vector depending on the dimensionality of the problem. This removes the time dependence from the partial differential equation, making it sufficient to solve a (pseudo-)steady-state problem including the unknown scalar $s$, the allowed values of which are determined implicitly by the boundary conditions and problem parameters. The resulting equation will be a partial differential equation in space, but polynomial with respect to $s$.

The trial function (3.1) produces a complex-valued solution $w(x, t)$. The space component $W(x)$ is typically real-valued for stationary materials, and complex-valued for moving materials. It is easy to see that in the case of linear partial differential equations with real-valued coefficients, the real and imaginary components of $w(x, t)$ will also be solutions of the original problem. Let $\mathcal{L}$ be a linear differential operator. For example, for the real part, we have

$$
\begin{aligned}
\operatorname{Re}(\mathcal{L}(w)) & =\operatorname{Re}[\mathcal{L}(\operatorname{Re}(w)+i \operatorname{Im}(w))] \\
& =\operatorname{Re}[\mathcal{L}(\operatorname{Re}(w))+i \mathcal{L}(\operatorname{Im}(w))] \\
& =\mathcal{L}(\operatorname{Re}(w))
\end{aligned}
$$

where the last equality holds only if the coefficients of $\mathcal{L}$ are real. The same observation holds for the imaginary part. Thus, both $\operatorname{Re} w(x, t)$ and $\operatorname{Im} w(x, t)$ are real-valued solutions of the original problem.

However, for moving materials, the real and imaginary components of $W(x)$ are typically not solutions of the auxiliary steady-state problem: using the trial function (3.1), only the full complex-valued solution $W(x)$ is valid for the auxiliary problem. It is only the complete solution $w(x, t)$ whose real and imaginary components satisfy the original problem separately. For an example of this, the properties stated here can be easily verified for the analytical free vibration solution given in Sect. 2.1.1 for the constant-coefficient travelling string. The reason is, of course, that the stability exponent $s$ is complex. 
The allowed values of the stability exponent $s$ completely characterize the free vibrations of the elastic linear system under consideration. Consider the problem parameters fixed. If Re $s \leq 0$ for all solutions $(s, W)$, the system is stable and undergoes time-harmonic vibration. If $\operatorname{Re} s<0$ for one or more solutions $(s, W)$, these solutions also contain a damping component. If $\operatorname{Re} s>0$ for at least one solution $(s, W)$, the system is unstable. (Bolotin 1963)

Based on the trial function (3.1) we can make the distinction of static versus dynamic instability, depending on whether $\operatorname{Im} s=0$ in the critical state. This classification is due to Bolotin (1963). The concepts are illustrated in Fig. 3.1.

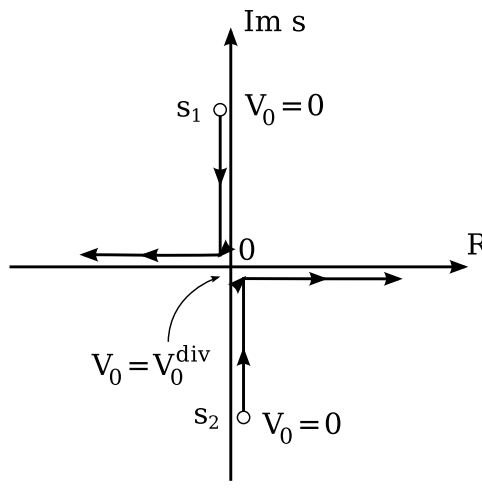

Static (divergence)

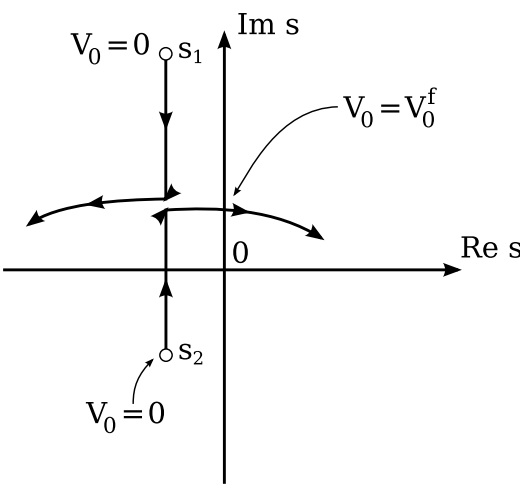

Dynamic (flutter)

Fig. 3.1 Behaviour of the stability exponent $s$ for the two different instability types in the classification due to Bolotin, 1963. The arrows show the motion of the eigenvalues $s_{j}$ as the problem parameter $V_{0}$ is increased quasistatically. In the left picture, the symbols are drawn off the axes for legibility reasons only; $s^{2} \in \mathbb{R}$ for all $V_{0}$. In the right picture, the real part is initially negative. In both cases, the eigenvalues merge at the collision point, and then immediately separate.

Roughly speaking, the critical state can be defined as follows. We begin in some initially stable state of the system. For axially travelling materials, the initial state is usually taken as axial velocity $V_{0}=0$. We then start increasing the problem parameter of interest, call it $p$, quasistatically. After a while, the parameter $p$ has reached a value $p_{0}$. If there exists at least one solution $(s, W)$ such that $\operatorname{Re} s>0$ for all $p=p_{0}+\varepsilon$ with arbitrarily small $\varepsilon>0$, but $\operatorname{Re} s \leq 0$ for all solutions $s$ for $p=p_{0}-\varepsilon$, the value $p_{0}$ is called the critical parameter value. It is the point of transition from stable to unstable behaviour.

Assume we have found a critical parameter value $p=p_{0}$. If $\operatorname{Im} s=0$ just above the critical value, $p=p_{0}+\varepsilon$, the complex-valued exp in (3.1) simplifies to a real-valued exp, and the displacement will grow exponentially with time. This is called static instability or divergence (Fig. 3.1, left). This corresponds to $s$ passing through the origin of the complex plane, and thus the critical 
states for this kind of instability can be found by a steady-state analysis (see Bolotin 1963, or consider (3.1) for $s=0$ ).

Often the existence of a nontrivial steady-state solution is taken as indication of an instability, and e.g. the buckling analysis of travelling panels and plates is based on this idea. However, in their analysis of the travelling ideal string, Wang et al (2005) caution that steady-state solutions may exist without indicating an instability, if the eigenfunctions remain linearly independent at the critical parameter value. Thus, we may conclude that a static instability can only arise from a steady state, but in a rigorous analysis, the existence of a steady state should be taken only as a necessary condition for static instability, not a sufficient one.

If $\operatorname{Im} s \neq 0$ for $p=p_{0}+\varepsilon$, the complex-valued exp in (3.1) becomes a product of a real-valued exp and a harmonic component, e.g. sin, cos or a linear combination of these. In this case, the displacement will exhibit exponentially growing vibrations with time (see Fig. 3.1, right). This is called dynamic instability or flutter. This should not be confused with the engineering use of the term flutter to describe also stable vibrations.

Linear perturbation analysis around the critical parameter value is one method that can be used to confirm that the critical state indeed indicates an instability for $p=p_{0}+\varepsilon$ (see e.g. the analysis of Parker 1998, for the moving beam). Another method is to compute the complex eigenfrequencies of the system, based on the trial function (3.1), for a range of parameter values $\left[p_{0}-\varepsilon, p_{0}+\varepsilon\right]$.

In the case of stability analysis of linear partial differential equations, it is evident from the linear superposition property that solution components which obviously always stay bounded may be discarded without further consideration. Thus, linear stability analysis can be focused on the solution components for which the boundedness of the long-term behaviour, under various different values for the problem parameters, is nontrivial.

Finally, it should be noted that for the investigation of small vibrations of elastic systems, linearized models are often used. If system enters an unstable state, the small displacement assumption eventually breaks, possibly very quickly. From that point on, the model no longer describes the physics of the situation being analyzed. It is generally agreed that linearized smalldisplacement models are sufficient up to the first instability (see e.g. Païdoussis 2005).

\subsection{Dynamic analysis of moving membranes and plates}

From experimental studies and some theoretical estimations, it is known that mechanical instability of a travelling paper web can arise at some critical velocities, and that the instability may occur in either dynamic, i.e. flutter, or static, i.e. divergence, forms. These critical velocities are of both theoretical 
and practical interest, as they set an upper limit for the running speed of paper machines, and consequently, for the rate of paper production that can be achieved. Some previous investigations show that for an axially moving elastic paper web under a homogeneous tension profile along the rollers and certain other conditions, the value of divergence speed $V_{0}^{\text {div }}$ is smaller than the value of flutter speed $V_{0}^{\mathrm{f}}$, and hence the critical instability will be of the divergence type.

In this section, the focus is on how to analyze the dynamic problem of stability. We will follow the method described by Bolotin (1963). Recall the equation of small transverse vibrations of the travelling plate subjected to homogeneous tension, (2.12). We represent it as follows:

$$
\frac{\partial^{2} w}{\partial t^{2}}+2 V_{0} \frac{\partial^{2} w}{\partial x \partial t}+\left(V_{0}^{2}-C^{2}\right) \frac{\partial^{2} w}{\partial x^{2}}+\frac{D_{0}}{m} \mathcal{L}_{0}(w)=0, \quad C=\sqrt{\frac{T_{0}}{m}},
$$

where $w=w(x, t)$ is the transverse displacement, and the orthotropic bending operator is

$$
\mathcal{L}_{0}(w)=\frac{D_{1}}{D_{0}} \frac{\partial^{4} w}{\partial x^{4}}+\frac{2 D_{3}}{D_{0}} \frac{\partial^{4} w}{\partial x^{2} \partial y^{2}}+\frac{D_{2}}{D_{0}} \frac{\partial^{4} w}{\partial y^{4}} .
$$

Here $D_{j}$ for $j=1,2,3$ are the orthotropic bending rigidities

$$
D_{1}=\frac{h^{3}}{12} C_{11}, \quad D_{2}=\frac{h^{3}}{12} C_{22}, \quad D_{3}=\frac{h^{3}}{12}\left(C_{12}+2 C_{66}\right),
$$

which were already introduced as (2.18), Sect. 2.1.3 (or see Timoshenko and Woinowsky-Krieger 1959, chap. 11). The $C_{i j}$ are the elastic moduli, (2.19). The quantity $D_{0}$ is a normalization constant, for which we have chosen the value $D_{0}=D_{1}$.

The boundary value problem consisting of (3.3-3.4) with the boundary conditions $(2.20-2.22)$ is homogeneous and invariant with respect to the symmetry operation $y \rightarrow-y$ and, consequently, all solutions of the problem are either symmetric or antisymmetric functions of $y$, i.e.

$$
w(x, y, t)=w(x,-y, t) \quad \text { or } \quad w(x, y, t)=-w(x,-y, t) .
$$

In the following analysis, however, this symmetry property is not necessary.

Using the time-harmonic trial function (3.1), we can represent the solution of our dynamic boundary-value problem (3.3-3.4), (2.20-2.22) as

$$
w(x, y, t)=W(x, y) e^{i \omega t}=W(x, y) e^{s t},
$$

where $\omega$ is the angular frequency of small transverse vibrations and $s=i \omega$ is the stability exponent. As presented in the previous section, Sect. 3.2, if $s$ is purely imaginary and consequently $\omega$ is real, the membrane or plate performs harmonic vibrations of a small amplitude and its motion can be considered stable. If, for some values of the problem parameters, the real 
part of the stability exponent becomes positive, the transverse vibrations grow exponentially and consequently the behaviour is unstable (See Fig. 3.1, left).

\subsubsection{Dynamic stability of membranes}

Classical approach for modelling of moving materials is to apply the model of a travelling membrane. In the case of a membrane, efficient analytical methods are usually available. We begin the analysis of the moving membrane by defining the corresponding eigenvalue problem. Homogeneous tension is applied at the boundaries $x=0$ and $x=\ell$. In order to investigate the dynamic behavior, we insert the representation (3.6) into (3.3). Since the case of a membrane is considered, we omit the bending rigidity terms from (3.3), i.e., $\left(D_{0} / m\right) \mathcal{L}_{0}(w)=0$. We obtain the following equation for small time-harmonic vibrations of the travelling membrane:

$$
s^{2} W+2 s V_{0} \frac{\partial W}{\partial x}+\left(V_{0}^{2}-C^{2}\right) \frac{\partial^{2} W}{\partial x^{2}}=0,
$$

with zero displacement boundary conditions

$$
(W)_{x=0, \ell}=0, \quad-b \leq y \leq b .
$$

We will see that the choice of boundary conditions in the $y$ direction, on the edges $\{0 \leq x \leq \ell, y= \pm b\}$, does not matter in the following analysis.

We multiply (3.7) by $W$ and perform integration over the domain

$$
\Omega \equiv\left\{(x, y) \in \mathbb{R}^{2} \mid 0<x<\ell,-b<y<b\right\}
$$

to obtain

$$
s^{2} \int_{\Omega} W^{2} \mathrm{~d} \Omega+2 s V_{0} \int_{\Omega} W \frac{\partial W}{\partial x} \mathrm{~d} \Omega+\left(V_{0}^{2}-C^{2}\right) \int_{\Omega} W \frac{\partial^{2} W}{\partial x^{2}} \mathrm{~d} \Omega=0 .
$$

It is worth noting that the problem (3.10) is a special case of the variational form of the original eigenvalue problem (3.7). In (3.10), we only test against $W$ itself, not against an arbitrary test function. However, any solution of the original problem (3.7) is also a solution of (3.10). Hence, the eigenvalues of problem (3.10) include the eigenvalues of problem (3.7), i.e. we may get additional solutions. As we are interested in the behavior of the eigenvalues of problem (3.7), it is sufficient to notice that if all the eigenvalues of problem (3.10) have similar behaviour with each other, then the eigenvalues of (3.7) have the same behaviour.

The second and third integrals in (3.10) are evaluated with integration by parts and the boundary conditions (3.8): 


$$
\begin{aligned}
\int_{\Omega} W \frac{\partial W}{\partial x} \mathrm{~d} \Omega & =\int_{-b}^{b} \int_{0}^{\ell} W \frac{\partial W}{\partial x} \mathrm{~d} x \mathrm{~d} y \\
& =\int_{-b}^{b}\left[\frac{W^{2}(\ell, y)}{2}-\frac{W^{2}(0, y)}{2}\right] \mathrm{d} y \\
& =\quad 0,
\end{aligned}
$$

and

$$
\int_{\Omega} W \frac{\partial^{2} W}{\partial x^{2}} \mathrm{~d} \Omega=-\int_{\Omega}\left(\frac{\partial W}{\partial x}\right)^{2} \mathrm{~d} \Omega .
$$

Using (3.10-3.12) and performing elementary transformations, we obtain the following expression for the stability exponent :

$$
s^{2}=\left(V_{0}^{2}-C^{2}\right) \frac{\int_{\Omega}\left(\frac{\partial W}{\partial x}\right)^{2} \mathrm{~d} \Omega}{\int_{\Omega} W^{2} \mathrm{~d} \Omega} .
$$

If $s$ becomes zero, we have a steady state solution (divergence) with frequency $\omega=0$ at the velocity $V_{0}=V_{0}^{\text {div }}$. From (3.13), the value of this divergence velocity is found as

$$
V_{0}^{\mathrm{div}}=C=\sqrt{\frac{T_{0}}{m}}=\sqrt{\frac{h u_{0}}{m \ell} E_{1}},
$$

where in the last form, (2.40) from Sect. 2.2 has been used. Here $u_{0}$ is a prescribed displacement at $x=\ell$.

\subsubsection{Dynamic analysis of small transverse vibrations and elastic stability of isotropic plates}

To investigate the dynamic behaviour of the plate, we insert, following the membrane case, representation (3.6) into (3.3). As the object is a plate, the bending rigidities cannot be omitted. Therefore, for small time-harmonic vibrations of the travelling plate subjected to homogeneous tension, we have the equation

$$
s^{2} W+2 s V_{0} \frac{\partial W}{\partial x}+\left(V_{0}^{2}-C^{2}\right) \frac{\partial^{2} W}{\partial x^{2}}+\frac{D}{m} \Delta^{2} W=0 .
$$

Boundary conditions for the plate problem are 


$$
\begin{array}{rlr}
(W)_{x=0, \ell}=0, \quad\left(\frac{\partial^{2} W}{\partial x^{2}}\right)_{x=0, \ell}=0, & -b \leq y \leq b, \\
\left(\frac{\partial^{2} W}{\partial y^{2}}+\beta_{1} \frac{\partial^{2} W}{\partial x^{2}}\right)_{y= \pm b}=0, & 0 \leq x \leq \ell, \\
\left(\frac{\partial^{3} W}{\partial y^{3}}+\beta_{2} \frac{\partial^{3} W}{\partial x^{2} \partial y}\right)_{y= \pm b}=0, & 0 \leq x \leq \ell .
\end{array}
$$

For an orthotropic plate, we have

$$
\begin{gathered}
\beta_{1}=\nu_{12}, \\
\beta_{2}=\nu_{12}+\frac{4 G_{12}}{E_{2}}\left(1-\nu_{12} \nu_{21}\right) .
\end{gathered}
$$

As was noted in Sect. 2.1.3, in the case of an isotropic plate, the parameters above become simplified as

$$
\beta_{1}=\nu \quad \text { and } \quad \beta_{2}=2-\nu,
$$

by setting $\nu_{12}=\nu_{21}=\nu, E_{1}=E_{2}=E, G_{12}=G$, and using the isotropic shear modulus relation $G=E /(2(1+\nu))$. Then, factoring $1-\nu_{12} \nu_{21}=$ $1-\nu^{2}=(1+\nu)(1-\nu)$ and simplifying reduces (3.19) into (3.20).

Proceeding similarly as in the membrane case, we multiply (3.15) by $W$ and perform integration over the domain $\Omega$ to obtain

$$
\begin{aligned}
s^{2} \int_{\Omega} W^{2} \mathrm{~d} \Omega+2 s V_{0} \int_{\Omega} W \frac{\partial W}{\partial x} \mathrm{~d} \Omega+\left(V_{0}^{2}-C^{2}\right) \int_{\Omega} W \frac{\partial^{2} W}{\partial x^{2}} \mathrm{~d} \Omega & \\
+\frac{D}{m} \int_{\Omega} W \Delta^{2} W \mathrm{~d} \Omega & =0 .
\end{aligned}
$$

The same argument holds for the variational form as in the membrane case. Equation (3.21) can be seen as an eigenvalue problem for the pair $(s, W)$ with the parameter $V_{0}$, producing a spectrum of complex eigenfrequencies $s$ and eigenmodes $W$ for the chosen value of $V_{0}$. Alternatively, (3.21) can be viewed as an eigenvalue problem for the pair $\left(V_{0}, W\right)$ with the parameter $s$, when $s$ is fixed to any such value that at least one complex eigenfrequency exists for at least one choice of $V_{0}$. For other choices of $s$, this second eigenvalue problem has no solution.

Previously, we have noted the equations (3.11) and (3.12) for the membrane. By using Green's 2nd identity, the last integral in (3.21) can be transformed into

$$
\int_{\Omega} W \Delta^{2} W \mathrm{~d} \Omega=\int_{\Omega}(\Delta W)^{2} \mathrm{~d} \Omega+\int_{\Gamma}\left(W \frac{\partial}{\partial n} \Delta W-\Delta W \frac{\partial W}{\partial n}\right) \mathrm{d} \Gamma,
$$


where $n$ is the exterior unit normal to the boundary $\Gamma$ of the domain $\Omega$. We

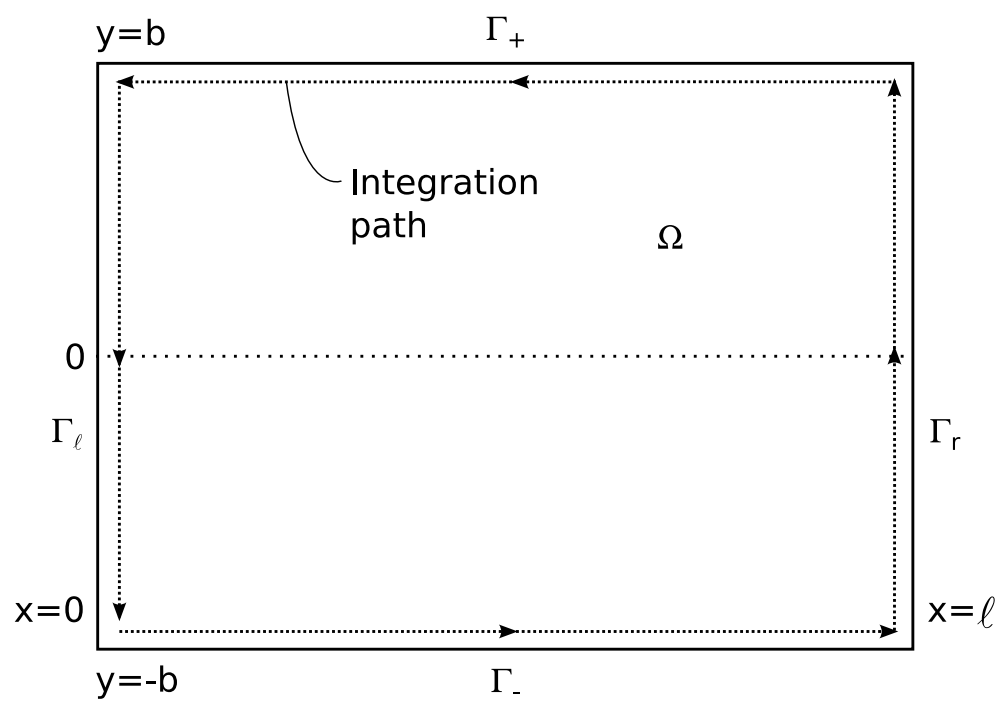

Fig. 3.2 Division of the boundary $\Gamma$ for the investigated contour integral.

divide the boundary $\Gamma$ into four parts (see Fig. 3.2):

$$
\begin{array}{cc}
\Gamma_{-}=\{0 \leq x \leq \ell, y=-b\}, & \Gamma_{r}=\{x=\ell,-b \leq y \leq b\}, \\
\Gamma_{+}=\{0 \leq x \leq \ell, y=b\}, & \Gamma_{\ell}=\{x=0,-b \leq y \leq b\} .
\end{array}
$$

Admitting counterclockwise integration along $\Gamma$, we have

$$
I=\int_{\Gamma}\left(W \frac{\partial}{\partial n} \Delta W-\Delta W \frac{\partial W}{\partial n}\right) \mathrm{d} \Gamma=I_{-}+I_{r}+I_{+}+I_{\ell} .
$$

Here

$$
\begin{gathered}
I_{r}=I_{\ell}=0 \\
I_{-}=\int_{\Gamma_{-}}\left(W \frac{\partial}{\partial n} \Delta W-\Delta W \frac{\partial W}{\partial n}\right) \mathrm{d} \Gamma \\
=-\int_{0}^{\ell}\left(W \frac{\partial}{\partial y} \Delta W-\Delta W \frac{\partial W}{\partial y}\right)_{y=-b} \mathrm{~d} x,
\end{gathered}
$$




$$
\begin{aligned}
I_{+} & =\int_{\Gamma_{+}}\left(W \frac{\partial}{\partial n} \Delta W-\Delta W \frac{\partial W}{\partial n}\right) \mathrm{d} \Gamma \\
& =-\int_{\ell}^{0}\left(W \frac{\partial}{\partial y} \Delta W-\Delta W \frac{\partial W}{\partial y}\right)_{y=b} \mathrm{~d} x \\
& =\int_{0}^{\ell}\left(W \frac{\partial}{\partial y} \Delta W-\Delta W \frac{\partial W}{\partial y}\right)_{y=b} \mathrm{~d} x
\end{aligned}
$$

where we have used the relations

$$
\begin{aligned}
& \mathrm{d} \Gamma=\mathrm{d} x, \quad \frac{\partial}{\partial n}=-\frac{\partial}{\partial y} \quad \text { for }(x, y) \in \Gamma_{-}, \\
& \mathrm{d} \Gamma=-\mathrm{d} x, \quad \frac{\partial}{\partial n}=\frac{\partial}{\partial y} \quad \text { for }(x, y) \in \Gamma_{+},
\end{aligned}
$$

and

$$
W=\Delta W=0 \quad \text { for }(x, y) \in \Gamma_{\ell}+\Gamma_{r} .
$$

We obtain

$$
I=I_{-}+I_{+}=\int_{0}^{\ell}\left(Q(W, W)_{y=b}-Q(W, W)_{y=-b}\right) \mathrm{d} x,
$$

where

$$
Q(w, v) \equiv v \frac{\partial}{\partial y} \Delta w-\Delta w \frac{\partial v}{\partial y} .
$$

with the arbitrary functions $v$ and $w$. Using the boundary conditions for an isotropic plate, (3.17) and (3.18), we find that

$$
Q(W, W)=\frac{W \frac{\partial^{3} W}{\partial y^{3}}}{\left(\frac{2-\nu}{1-\nu}\right)}+\frac{\frac{\partial W}{\partial y} \frac{\partial^{2} W}{\partial y^{2}}}{\left(\frac{\nu}{1-\nu}\right)}, \quad \text { at } y= \pm b
$$

We can see from (3.32) that the function $Q$ is antisymmetric with respect to the transformation $y \rightarrow-y$ for symmetric and antisymmetric functions $W$, and consequently,

$$
Q(W, W)_{y=b}=-Q(W, W)_{y=-b} .
$$

We observe that

$$
I=2 \int_{0}^{\ell} Q(W, W)_{y=b} \mathrm{~d} x .
$$

From (3.22) and (3.34), we obtain 


$$
\int_{\Omega} W \Delta^{2} W \mathrm{~d} \Omega=\int_{\Omega}(\Delta W)^{2} \mathrm{~d} \Omega+2 \int_{0}^{\ell} Q(W, W)_{y=b} \mathrm{~d} x,
$$

and, furthermore

$$
\begin{aligned}
\omega^{2} & =-s^{2} \\
= & \frac{\left(C^{2}-V_{0}^{2}\right) \int_{\Omega}\left(\frac{\partial W}{\partial x}\right)^{2} \mathrm{~d} \Omega+\frac{D}{m}\left(\int_{\Omega}(\Delta W)^{2} \mathrm{~d} \Omega+2 \int_{0}^{\ell} Q_{y=b} \mathrm{~d} x\right)}{\int_{\Omega} W^{2} \mathrm{~d} \Omega} .
\end{aligned}
$$

We can now observe from representation (3.36) the following equation for the divergence mode (buckling mode):

$$
\left(V_{0}^{\text {div }}\right)^{2}=C^{2}+\frac{D}{m} \frac{\int_{\Omega}(\Delta W)^{2} \mathrm{~d} \Omega+2 \int_{0}^{\ell} Q_{y=b} \mathrm{~d} x}{\int_{\Omega}\left(\frac{\partial W}{\partial x}\right)^{2} \mathrm{~d} \Omega} .
$$

In particular, it follows from (3.37) that when the bending rigidity $D$ is negligible, the critical velocity is the same as for the axially travelling string (see, e.g., Chang and Moretti 1991). From the result further above, see (3.14), we see that the same value for the critical velocity also applies to ideal membranes. For a membrane, the divergence velocity does not depend on $W$. Thus, any combination of modes may occur at the critical velocity for the special case of an ideal membrane under homogeneous tension. These observations generalize the analogous results for a cylindrical deformation, i.e. a flat panel model of an ideal membrane (see Banichuk et al 2010b).

\subsection{Divergence instability of isotropic plates}

Next we will consider the buckling problem of an axially moving isotropic plate. In many practical cases, this is a reasonable simplification. For divergence instability of axially moving orthotropic plates, see Sect. 3.5.

\subsubsection{Eigenvalue problem}

In this section, we will study the divergence (static instability) of a travelling isotropic plate subjected to homogeneous tension. The problem is formulated as an eigenvalue problem of the partial differential equation 


$$
\left(m V_{0}^{2}-T_{0}\right) \frac{\partial^{2} W}{\partial x^{2}}+D\left(\frac{\partial^{4} W}{\partial x^{4}}+2 \frac{\partial^{4} W}{\partial x^{2} \partial y^{2}}+\frac{\partial^{4} W}{\partial y^{4}}\right)=0
$$

with the boundary conditions (3.16-3.18) and (3.20) (Sect. 3.3.2). We will study static (i.e. divergence) instability, and therefore time-dependent terms are excluded from (3.3). In order to determine the minimal eigenvalue

$$
\lambda=\gamma^{2}=\frac{\ell^{2}}{\pi^{2} D}\left(m V_{0}^{2}-T_{0}\right)
$$

of the problem (3.16-3.18), (3.38), and the corresponding eigenfunction $W=$ $W(x, y)$, we apply the following representation:

$$
W=W(x, y)=f\left(\frac{y}{b}\right) \sin \left(\frac{\pi x}{\ell}\right),
$$

where $f(y / b)$ is an unknown function. It follows from (3.40) that the desired buckling mode (steady-state solution) $W$ satisfies the boundary condition (3.16). The half-sine shape of the solution in the longitudinal direction is wellknown (see, e.g., the article by Lin 1997). Using the dimensionless quantities

$$
\eta=\frac{y}{b}, \quad \mu=\frac{\ell}{\pi b},
$$

and the relations (3.17-3.18) (Sect. 3.3.2) and (3.38-3.41), we obtain the following eigenvalue problem for the unknown function $f(\eta)$ :

$$
\begin{gathered}
\mu^{4} \frac{\mathrm{d}^{4} f}{\mathrm{~d} \eta^{4}}-2 \mu^{2} \frac{\mathrm{d}^{2} f}{\mathrm{~d} \eta^{2}}+(1-\lambda) f=0, \quad-1<\eta<1, \\
\mu^{2} \frac{\mathrm{d}^{2} f}{\mathrm{~d} \eta^{2}}-\nu f=0, \quad \eta= \pm 1, \\
\mu^{2} \frac{\mathrm{d}^{3} f}{\mathrm{~d} \eta^{3}}-(2-\nu) \frac{\mathrm{d} f}{\mathrm{~d} \eta}=0, \quad \eta= \pm 1,
\end{gathered}
$$

where (3.43-3.44) represent the free-of-traction boundary conditions.

\subsubsection{Analytical solution}

In this section, we will present the solution process of the eigenvalue problem $(3.42-3.44)$. We consider the problem as a spectral boundary value problem. The problem is invariant with respect to the symmetry operation $\eta \rightarrow-\eta$, and consequently, all its eigenfunctions can be classified as

$$
f^{\mathrm{s}}(\eta)=f^{\mathrm{s}}(-\eta), \quad f^{\mathrm{a}}(\eta)=-f^{\mathrm{a}}(-\eta), \quad 0 \leq \eta \leq 1 .
$$


Here $f^{\mathrm{s}}$ and $f^{\mathrm{a}}$ are symmetric and antisymmetric (skew-symmetric) with respect to the $x$ axis $(\eta=0)$. When $\gamma \leq 1$, a divergence mode symmetric with respect to the $x$ axis can be presented in the form

$$
W=f^{\mathrm{s}}(\eta) \sin \left(\frac{\pi x}{\ell}\right)
$$

where

$$
f^{\mathrm{s}}(\eta)=A^{\mathrm{s}} \cosh \left(\frac{\kappa_{+} \eta}{\mu}\right)+B^{\mathrm{s}} \cosh \left(\frac{\kappa_{-} \eta}{\mu}\right)
$$

and

$$
\kappa_{+}=\sqrt{1+\gamma}, \quad \kappa_{-}=\sqrt{1-\gamma} .
$$

The function $f^{\mathrm{s}}(\eta)$ is a symmetric solution of (3.42), and $A^{\mathrm{s}}$ and $B^{\mathrm{s}}$ are arbitrary constants. At first, we concentrate on the symmetric case and return to the antisymmetric case later.

Using the relations (3.43-3.47), we can derive the linear algebraic equations for determining the constants $A^{\mathrm{s}}$ and $B^{\mathrm{s}}$ :

$$
\begin{gathered}
A^{\mathrm{s}}\left(\kappa_{+}^{2}-\nu\right) \cosh \left(\frac{\kappa_{+}}{\mu}\right)+B^{\mathrm{s}}\left(\kappa_{-}^{2}-\nu\right) \cosh \left(\frac{\kappa_{-}}{\mu}\right)=0, \\
-A^{\mathrm{s}} \kappa_{+}\left(\kappa_{-}^{2}-\nu\right) \sinh \left(\frac{\kappa_{+}}{\mu}\right)-B^{\mathrm{s}} \kappa_{-}\left(\kappa_{+}^{2}-\nu\right) \sinh \left(\frac{\kappa_{-}}{\mu}\right)=0 .
\end{gathered}
$$

The condition for a non-trivial solution to exist in the form (3.46-3.48) is that the determinant of the system (3.49-3.50) must vanish. This is seen by observing that (3.49-3.50) is a homogeneous system of linear equations in $A^{\mathrm{s}}, B^{\mathrm{s}}$ :

$$
\left[\begin{array}{ll}
K_{11} & K_{12} \\
K_{21} & K_{22}
\end{array}\right]\left[\begin{array}{l}
A^{\mathrm{s}} \\
B^{\mathrm{s}}
\end{array}\right]=\left[\begin{array}{l}
0 \\
0
\end{array}\right]
$$

where the coefficients $K_{i j}$ are given by the obvious identifications. From linear algebra, it is known that a non-trivial solution satisfying (3.51) can only exist if the matrix $\mathbf{K}$ is singular. Hence its determinant must be zero.

This zero determinant condition leads to the transcendental equation

$$
\begin{aligned}
\kappa_{-}\left(\kappa_{+}^{2}-\nu\right)^{2} \cosh \left(\frac{\kappa_{+}}{\mu}\right) \sinh \left(\frac{\kappa_{-}}{\mu}\right)- \\
\kappa_{+}\left(\kappa_{-}^{2}-\nu\right)^{2} \sinh \left(\frac{\kappa_{+}}{\mu}\right) \cosh \left(\frac{\kappa_{-}}{\mu}\right)=0,
\end{aligned}
$$

which determines the eigenvalues 


$$
\lambda=\gamma^{2}
$$

implicitly. Equation (3.52) can be transformed into a more convenient form,

$$
\Phi(\gamma, \mu)-\Psi(\gamma, \nu)=0,
$$

where we have defined

$$
\Phi(\gamma, \mu)=\tanh \left(\frac{\sqrt{1-\gamma}}{\mu}\right) \operatorname{coth}\left(\frac{\sqrt{1+\gamma}}{\mu}\right)
$$

and

$$
\Psi(\gamma, \nu)=\frac{\sqrt{1+\gamma}}{\sqrt{1-\gamma}} \frac{(\gamma+\nu-1)^{2}}{(\gamma-\nu+1)^{2}} .
$$

Let us consider the modes of buckling which are antisymmetric about the $x$ axis:

$$
W=f^{\mathrm{a}}(\eta) \sin \left(\frac{\pi x}{\ell}\right),
$$

where

$$
f^{\mathrm{a}}(\eta)=A^{\mathrm{a}} \sinh \left(\frac{\kappa_{+} \eta}{\mu}\right)+B^{\mathrm{a}} \sinh \left(\frac{\kappa_{-} \eta}{\mu}\right)
$$

for $\gamma \leq 1$. The values $\kappa_{+}$and $\kappa_{-}$are defined by the expressions (3.48). Using the expression (3.58) for $f^{\text {a }}$ and the boundary conditions on the free edges of the plate (3.43-3.44), we obtain the following transcendental equation for determining the quantity $\gamma$ :

$$
\Phi(\gamma, \mu)-\frac{1}{\Psi(\gamma, \nu)}=0 .
$$

In (3.59), $\Phi(\gamma, \mu)$ and $\Psi(\gamma, \nu)$ are again defined by the formulas (3.55) and (3.56). In the segment $0<\gamma \leq 1$ being considered, the equation has two roots,

$$
\gamma=\gamma_{1} \quad \rightarrow \quad \gamma_{0}<\gamma_{1}<1
$$

and

$$
\gamma=\gamma_{2} \quad \rightarrow \quad \gamma_{2}=1
$$

for arbitrary values of the Poisson ratio $\nu$ and the geometric parameter $\mu$. By using (3.60-3.61) and some properties described in the next section, it is possible to determine that

$$
\gamma_{*}<\gamma_{1}<\gamma_{2},
$$

where $\gamma_{*}$ is the minimal eigenvalue for the symmetric case. Thus, the critical buckling mode is symmetric with respect to the $x$ axis, and corresponds to $\gamma=\gamma_{*}$, i.e., to the solution of (3.54). Hence we will limit our consideration to the symmetric case. 
We have obtained an equation determining the minimal eigenvalue $\gamma_{*}$, (3.54). By relation (3.39), the corresponding critical velocity of the travelling band is then represented as

$$
\left(V_{0}^{\mathrm{div}}\right)^{2}=\frac{T_{0}}{m}+\frac{\gamma_{*}^{2}}{m}\left(\frac{\pi^{2} D}{\ell^{2}}\right) .
$$

In order to obtain the corresponding eigenmode, either $A^{\mathrm{s}}$ or $B^{\mathrm{s}}$ can be solved from either of the equations (3.49-3.50), and the other one (either $B^{\mathrm{s}}$ or $A^{\mathrm{s}}$, respectively) can be chosen arbitrarily; it is the free coefficient of the eigenvalue problem. Finally, inserting the obtained $\gamma_{*}, A^{\mathrm{s}}$ and $B^{\mathrm{s}}$ into (3.46-3.47) gives the eigenmode corresponding to the eigenvalue $\gamma_{*}$.

One of $A^{\mathrm{s}}$ or $B^{\mathrm{s}}$ is left free, because the zero determinant condition holds at the value of $\gamma=\gamma_{*}$ that is a solution of (3.54). Hence, at $\gamma=\gamma_{*}$, the equations (3.49-3.50) become linearly dependent, providing only one condition.

\subsubsection{Properties of analytical solution}

In this section we investigate the properties of the functions $\Phi(\gamma, \mu)$ and $\Psi(\gamma, \nu)$, expressed by $(3.55-3.56)$, when $0 \leq \gamma \leq 1$. Their schematic illustration is presented in Fig. 3.3. As $\gamma$ increases from zero to unity, the function

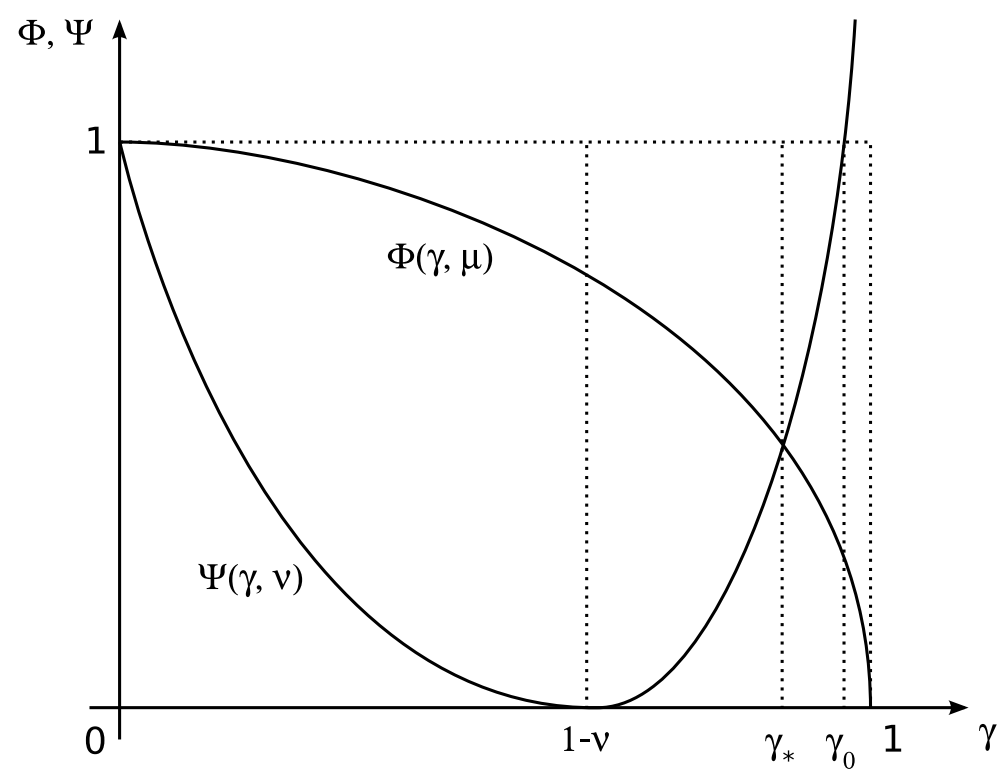

Fig. 3.3 Behavior of functions $\Phi$ and $\Psi$ with respect to the parameter $\gamma$ in the isotropic case. The presentation is qualitative. 
$\Phi(\gamma, \mu)$ decreases continuously and monotonically from 1 to 0 , i.e.

$$
1 \geq \Phi(\gamma, \mu) \geq 0, \quad \frac{\partial \Phi(\gamma, \mu)}{\partial \gamma}<0, \quad 0 \leq \gamma \leq 1
$$

and

$$
\begin{gathered}
\Phi(0, \mu)=\left(\tanh \frac{\sqrt{1-\gamma}}{\mu} \operatorname{coth} \frac{\sqrt{1+\gamma}}{\mu}\right)_{\gamma=0}=1 \\
\Phi(1, \mu)=\left(\tanh \frac{\sqrt{1-\gamma}}{\mu} \operatorname{coth} \frac{\sqrt{1+\gamma}}{\mu}\right)_{\gamma=1}=0 .
\end{gathered}
$$

The proof of the monotonical decrease of function $\Phi$ is performed in Sect. 3.5.5, where we investigate the solution of the orthotropic problem. The same property for the present isotropic problem follows as a special case.

The function $\Psi(\gamma, \nu)$ decreases from 1 to 0 in the interval $0<\gamma<1-\nu$,

$$
1>\Psi(\gamma, \nu)>0, \quad \frac{\partial \Psi(\gamma, \nu)}{\partial \gamma}<0, \quad 0<\gamma<1-\nu,
$$

and at the ends of this interval, we have

$$
\begin{gathered}
\Psi(0, \nu)=\left[\frac{\sqrt{1+\gamma}}{\sqrt{1-\gamma}} \frac{(\gamma+\nu-1)^{2}}{(\gamma-\nu+1)^{2}}\right]_{\gamma=0}=1, \\
\Psi(1-\nu, \nu)=\left[\frac{\sqrt{1+\gamma}}{\sqrt{1-\gamma}} \frac{(\gamma+\nu-1)^{2}}{(\gamma-\nu+1)^{2}}\right]_{\gamma=1-\nu}=0 .
\end{gathered}
$$

The function $\Psi$ increases monotonically in the interval $1-\nu<\gamma<1$, increasing without limit as $\gamma \rightarrow 1$, i.e.

$$
0<\Psi(\gamma, \nu)<\infty, \quad \frac{\partial \Psi(\gamma, \nu)}{\partial \gamma}>0, \quad 1-\nu<\gamma<1
$$

and

$$
\lim _{\gamma \rightarrow 1} \Psi(\gamma, \nu)=\infty .
$$

The limit (3.71) will be shown in Sect. 3.5.5.

Plots of the function $\Phi(\gamma, \mu)$ when the geometric aspect ratio $\ell / 2 b=0.1$, 1 , and 10 are shown in Fig. 3.4 at the top. The functions $\Psi(\gamma, \nu)$ when $\nu=0.2$, 0.3 and 0.5 are shown in the same figure, at the bottom.

The value of $\gamma=\gamma_{0}$, for which

$$
\Psi\left(\gamma_{0}, \nu\right)=1, \quad \gamma_{0} \in[1-\nu, 1]
$$

is of special interest. At this point it holds that 
$\Phi$

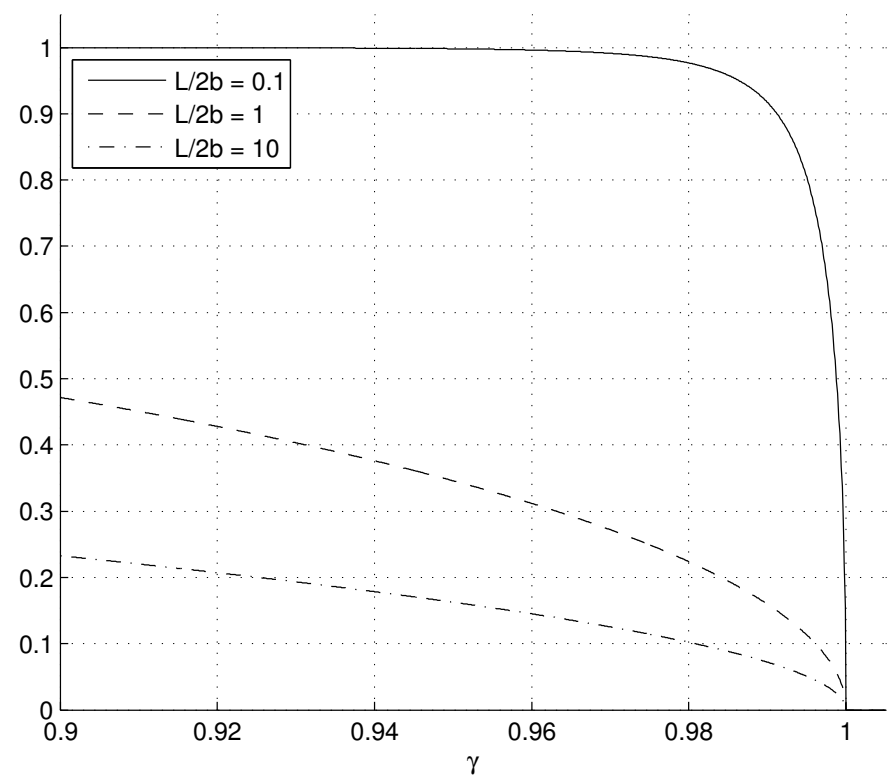

$\Psi$

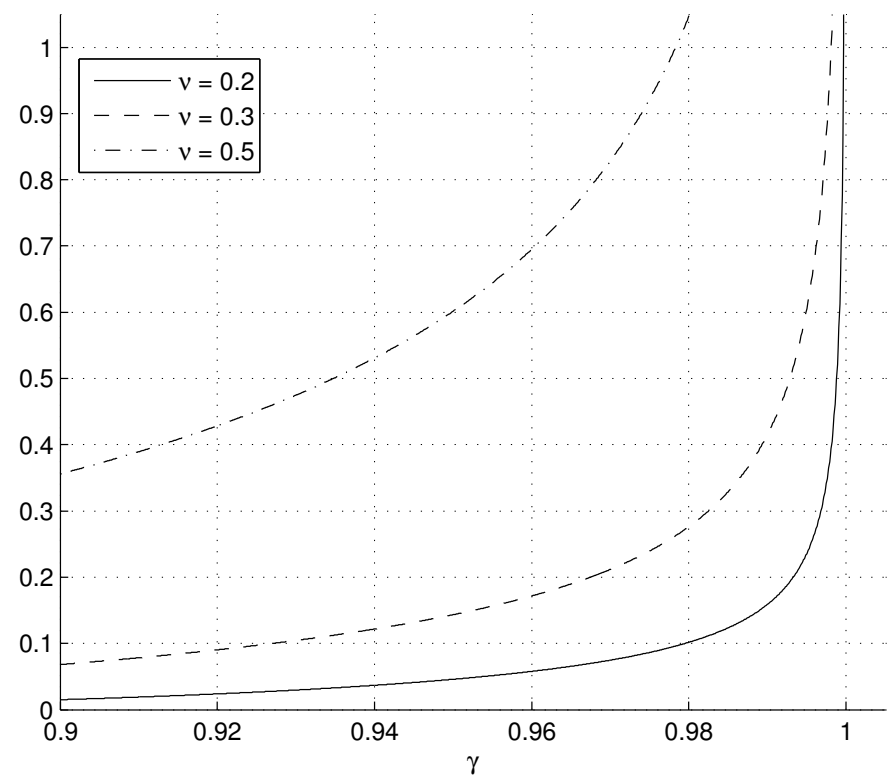

Fig. 3.4 Plots of $\Phi$ (top) and $\Psi$ (bottom) for different values of the parameters $\ell / 2 b$ and $\nu$. Note the horizontal scale. 


$$
\Phi-\Psi=\Phi-\frac{1}{\Psi} \quad \text { at } \quad \gamma=\gamma_{0}
$$

and hence the functions defined by the left-hand sides of (3.54) and (3.59) will cross at the value $\gamma=\gamma_{0}$.

The value $\gamma_{0}$ is found by inserting (3.56) into (3.72), squaring both sides for convenience (we know that $\gamma_{0}>0$, so no information is lost), and solving for $\gamma_{0}$. We obtain

$$
\gamma_{0}^{2}=(1-\nu)(3 \nu-1+2 \sqrt{1-2 \nu(1-\nu)})
$$

The other solutions are all negative and can thus be discarded. When examined as a function of $\nu$, the expression $\gamma_{0}(\nu)$ has zeros at $\nu=-3$ and $\nu=1$, and a maximum at $\nu=0$, with the value $\gamma_{0}=1$. For any other value of $\nu$, we have $\gamma_{0}<1$.

If we restrict the Poisson ratio $\nu$ into the physically admissible range for isotropic materials, $\nu \in(-1,0.5)$, then the value of $\gamma_{0}$, as given by (3.73), turns out to be close to unity. The minimal values are encountered at the ends of the range. At $\nu=-1$ we have $\gamma_{0} \approx 0.944$, and at $\nu=0.5, \gamma_{0} \approx 0.957$.

Let us consider the limiting cases in terms of the band geometry. First, if we have a long and narrow band span, $\ell \gg b$, the geometric parameter $\mu$ becomes large, and the arguments of tanh and coth in (3.55) become small. In such a case, we can use the following Taylor series expansions of the hyperbolic trigonometrics around $\alpha=0$ (here $\alpha$ is an arbitrary parameter):

$$
\begin{aligned}
& \tanh \alpha=\alpha-\frac{1}{3} \alpha^{3}+\frac{2}{15} \alpha^{5}+\ldots, \\
& \operatorname{coth} \alpha=\frac{1}{\alpha}+\frac{1}{3} \alpha-\frac{1}{45} \alpha^{3}+\ldots
\end{aligned}
$$

Retaining only the first term in each of (3.74), and applying to (3.55), we obtain the approximate expression

$$
\Phi=\sqrt{\frac{1-\gamma}{1+\gamma}} \quad(\text { for large } \mu) .
$$

Using (3.75) and (3.56) in (3.54), we find the solution

$$
\lambda_{\mathrm{e}}=\gamma_{\mathrm{e}}^{2}=1-\nu^{2}
$$

This solution corresponds to a narrow strip simply supported at its ends. It leads to the Euler value of the force for stability loss (buckling),

$$
P=P_{\mathrm{e}}=\lambda_{\mathrm{e}} \frac{\pi^{2} D}{\ell^{2}}=\pi^{2} \frac{E I}{\ell^{2}}
$$

where 


$$
P=m V_{0}^{2}-T_{0}, \quad D=\frac{E h^{3}}{12\left(1-\nu^{2}\right)}, \quad I=\frac{h^{3}}{12} .
$$

Furthermore, consider the Taylor expansion of $\cosh \alpha$ around $\alpha=0$ (here also $\alpha$ is an arbitrary parameter):

$$
\cosh \alpha=1+\frac{1}{2} \alpha^{2}+\frac{1}{24} \alpha^{4}+\ldots
$$

Retaining only the first term, we have a constant value. Looking at (3.47), which determines the corresponding mode of stability loss, we see that for large $\mu$, the dependence on $\eta$ thus vanishes, making the mode cylindrical.

With regard to both the critical load and the mode, we see that the case of a long, narrow strip corresponds to the classical one-dimensional case.

At the other extreme, for a very wide band for which $b \gg \ell$, we have $\mu \rightarrow 0$. In this case, we can use the limits

$$
\begin{gathered}
\lim _{\alpha \rightarrow \infty} \tanh \alpha=1, \\
\lim _{\alpha \rightarrow \infty} \operatorname{coth} \alpha=1,
\end{gathered}
$$

leading to

$$
\lim _{\mu \rightarrow 0^{+}} \Phi(\gamma, \mu)=1 .
$$

Using (3.80) and (3.56) in (3.54), we obtain the equation $\Psi(\gamma, \nu)=1$ at the limit $\mu \rightarrow 0$. Its solution is $\gamma=\gamma_{0}$, given by (3.73) above. Thus, if $\nu \neq 0$, it holds for the wide band that

$$
\gamma_{*} \rightarrow \gamma_{0} \neq 1 \quad \text { for } \quad \mu \rightarrow 0
$$

which differs from the classical one-dimensional value $\gamma_{\mathrm{e}}=\sqrt{1-\nu^{2}}$ given by (3.76). Numerically, it is seen that $\gamma_{0} \geq \gamma_{\mathrm{e}}$ for all $\nu \in(-1,0.5)$, where the equality holds only at $\nu=0$. Thus, the minimal eigenvalue in the limit of a wide band is almost always higher than the minimal eigenvalue of the classical one-dimensional case.

It also turns out that the corresponding mode of stability loss from $(3.46-$ 3.48 ) is not cylindrical. It is therefore seen that the case of a wide band does not reduce to the classical one-dimensional case.

For naturally occurring materials, for which $\nu \geq 0$, the largest difference between the critical parameter $\gamma_{*}$, which leads to the loss of stability of an infinitely wide band, and the corresponding value obtained assuming a distribution of the deflections in the form of cylindrical surface, occurs when $\nu=0.5$, i.e., in the case of an absolutely incompressible material. For auxetic materials, for which $\nu<0$, the largest difference occurs at the lower limit of the range, i.e. $\nu=-1$.

It follows from the above treatment and the properties of the functions $\Phi(\gamma, \mu)$ and $\Psi(\gamma, \nu)$ that the roots $\gamma=\gamma_{*}$ of (3.54) lie in the interval 


$$
\gamma_{\mathrm{e}} \leq \gamma_{*} \leq \gamma_{0}
$$

for all $0<\mu<\infty$. This is the property that is needed to complete the analysis of (3.62).

Some numerical examples of the divergence velocities, $V_{0}^{\text {div }}$, defined in (3.63), and the corresponding buckling modes will be given. The used values of physical parameters are given in Table 3.1. These parameters represent typical values of paper material and conditions of paper making.

Table 3.1 Physical parameters used in the numerical examples.

\begin{tabular}{llll}
\hline$T_{0}$ & $m$ & $h$ & $E$ \\
\hline $500 \mathrm{~N} / \mathrm{m}$ & $0.08 \mathrm{~kg} / \mathrm{m}^{2}$ & $10^{-4} \mathrm{~m}$ & $10^{9} \mathrm{~N} / \mathrm{m}^{2}$ \\
\hline
\end{tabular}

Fig. 3.5 shows some examples of the critical buckling modes (divergence modes), calculated with the help of relations in (3.46-3.48), (3.49) and (3.54$3.56)$, for different values of the aspect ratio $\ell /(2 b)$. We see a localisation phenomenon: most of the displacement in the buckling mode occurs near the free edges. This effect becomes more pronounced as the width of the plate increases with respect to its length.

Table 3.2 presents some example values of critical velocities $V_{0}^{\text {div }}$ of an axially moving isotropic plate. The row with $\nu=0.3$ corresponds to the plots in Fig. 3.5.

Table 3.2 Critical velocities, i.e. divergence velocities $V_{0}^{\text {div }}(\mathrm{m} / \mathrm{s})$ of an axially moving isotropic plate for selected values of Poisson ratio $\nu$ and the span length $\ell$. The width of the plate is $2 b=1 \mathrm{~m}$ in all cases. The other physical parameters used are given in Table 3.1. (Banichuk et al 2010a)

\begin{tabular}{lllll}
\hline & $\ell(\mathrm{m})$ & & & \\
\cline { 2 - 5 }$\nu$ & 10 & 1 & 0.1 & 0.01 \\
\hline 0.1 & 79.0569 & 79.0570 & 79.0635 & 79.7110 \\
0.3 & 79.0569 & 79.0570 & 79.0640 & 79.7659 \\
0.5 & 79.0569 & 79.0570 & 79.0652 & 79.8824 \\
\hline
\end{tabular}

\subsection{Divergence instability of orthotropic plates}

The ratio of Young's moduli, i.e. the degree of orthotropicity, defines the properties of the actual paper product, affecting its behaviour. Different degrees of orthotropicity are desired for different applications. Using an orthotropic 

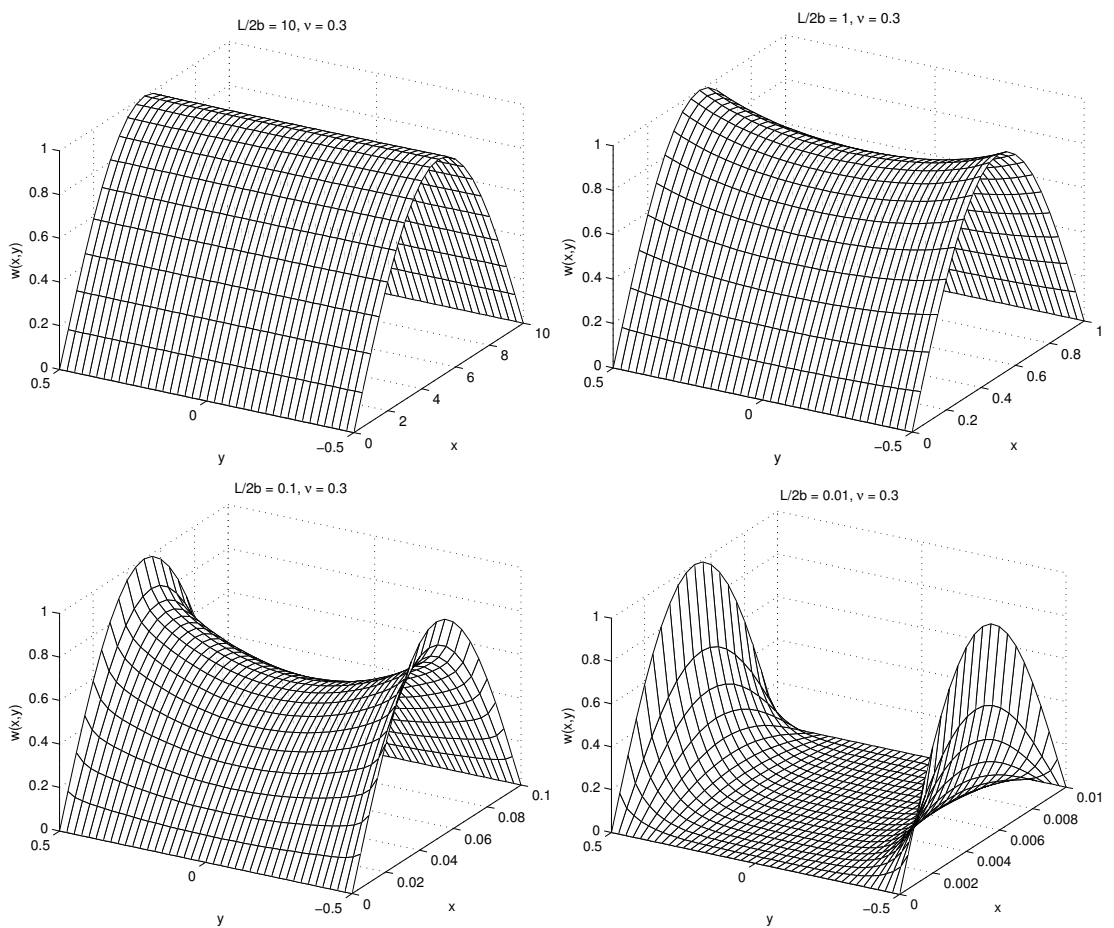

Fig. 3.5 Critical buckling modes (divergence modes) of an axially moving isotropic plate. Localization of deflections in the vicinity of the free boundaries can be seen, with the effect growing stronger as the aspect ratio $\ell / 2 b$ is decreased. (Reproduced from Banichuk et al 2010a)

material model, we can bring the analysis closer to the real life situation that is being modelled.

In this section, we will extend the results from Sect. 3.4 into the case of orthotropic materials.

\subsubsection{Eigenvalue problem}

The problem is formulated similarly to the isotropic eigenvalue problem, but now describing the divergence (static instability) of the travelling orthotropic plate subjected to homogeneous tension. We have the partial differential equation

$$
\left(m V_{0}^{2}-T_{0}\right) \frac{\partial^{2} W}{\partial x^{2}}+D_{0} \mathcal{L}_{0}(W)=0,
$$

with the boundary conditions (3.16-3.18); see Sect. 3.3.2, p. 46. Here the bending operator $\mathcal{L}_{0}(W)$ is 


$$
\mathcal{L}_{0}(W)=\frac{D_{1}}{D_{0}} \frac{\partial^{4} W}{\partial x^{4}}+\frac{2 D_{3}}{D_{0}} \frac{\partial^{4} W}{\partial x^{2} \partial y^{2}}+\frac{D_{2}}{D_{0}} \frac{\partial^{4} W}{\partial y^{4}},
$$

where the coefficients $D_{j}$ for $j=1,2,3$ are the orthotropic bending rigidities

$$
D_{1}=\frac{h^{3}}{12} C_{11}, \quad D_{2}=\frac{h^{3}}{12} C_{22}, \quad D_{3}=\frac{h^{3}}{12}\left(C_{12}+2 C_{66}\right),
$$

which were already introduced as (2.18), Sect. 2.1.3 (or see Timoshenko and Woinowsky-Krieger 1959, chap. 11). The $C_{i j}$ are the elastic moduli, (2.19). In (3.83) and (3.84), the coefficient $D_{0}$ is an arbitrary constant, which is convenient to take as $D_{0}=D_{1}$.

We wish to determine the minimal eigenvalue,

$$
\lambda=\gamma^{2}=\frac{\ell^{2}}{\pi^{2} D_{0}}\left(m V_{0}^{2}-T_{0}\right),
$$

of the problem (3.16-3.18) and (3.83-3.84). For the corresponding eigenfunction $W=W(x, y)$, we apply the same representation as before,

$$
W=W(x, y)=f\left(\frac{y}{b}\right) \sin \left(\frac{\pi x}{\ell}\right) .
$$

As was noted, the fact that the solution is a half-sine in the longitudinal direction is well-known in the isotropic case. It can be shown that the same form is applicable for the orthotropic plate. Again, what remains to be determined is the unknown cross-section $f(y / b)$.

It follows from (3.86) that the desired buckling form $W$ (steady-state solution) satisfies the boundary condition (3.16). Using the dimensionless quantities (same as before in Sect. 3.4)

$$
\eta=\frac{y}{b}, \quad \mu=\frac{\ell}{\pi b},
$$

and the relations (3.17-3.18) and (3.83-3.86), we obtain the following eigenvalue problem for the unknown function $f(\eta)$ :

$$
\begin{gathered}
\mu^{4} H_{2} \frac{\mathrm{d}^{4} f}{\mathrm{~d} \eta^{4}}-2 \mu^{2} H_{3} \frac{\mathrm{d}^{2} f}{\mathrm{~d} \eta^{2}}+\left(H_{1}-\lambda\right) f=0, \quad-1<\eta<1, \\
\mu^{2} \frac{\mathrm{d}^{2} f}{\mathrm{~d} \eta^{2}}-\beta_{1} f=0, \quad \eta= \pm 1, \\
\mu^{2} \frac{\mathrm{d}^{3} f}{\mathrm{~d} \eta^{3}}-\beta_{2} \frac{\mathrm{d} f}{\mathrm{~d} \eta}=0, \quad \eta= \pm 1,
\end{gathered}
$$

where $H_{1}, H_{2}$ and $H_{3}$ are dimensionless bending rigidities, defined by 


$$
H_{1}=\frac{D_{1}}{D_{0}}, \quad H_{2}=\frac{D_{2}}{D_{0}}, \quad H_{3}=\frac{D_{3}}{D_{0}},
$$

and $D_{0}$ is the characteristic bending rigidity, which is an arbitrary constant. In this book, we will use the choice $D_{0}=D_{1}$, which will be convenient in the calculations to follow. The parameters $\beta_{1}$ and $\beta_{2}$ are given by (3.19). Equations (3.89-3.90) represent the free-of-traction boundary conditions.

\subsubsection{Non-negativeness of eigenvalues}

To show that the eigenvalues $\lambda$ of the problem (3.88-3.90) are non-negative, one can proceed by using general ideas from Chen et al (1998), who proved a similar result for an isotropic stationary plate. Let us denote

$$
\mathcal{L}_{1}(f)=\mu^{4} H_{2} \frac{\mathrm{d}^{4} f}{\mathrm{~d} \eta^{4}}-2 \mu^{2} H_{3} \frac{\mathrm{d}^{2} f}{\mathrm{~d} \eta^{2}}+H_{1} f .
$$

We introduce the bilinear form $a(f, g)$ that corresponds to the strain energy of a plate (see e.g. Timoshenko and Woinowsky-Krieger 1959),

$$
\begin{aligned}
a(f, g)= & \int_{-1}^{1}\left[H_{1} f \bar{g}-\mu^{2} B_{1} f \frac{\mathrm{d}^{2} \bar{g}}{\mathrm{~d} \eta^{2}}-\mu^{2} B_{1} \frac{\mathrm{d}^{2} f}{\mathrm{~d} \eta^{2}} \bar{g}\right. \\
& \left.+\mu^{4} H_{2} \frac{\mathrm{d}^{2} f}{\mathrm{~d} \eta^{2}} \frac{\mathrm{d}^{2} \bar{g}}{\mathrm{~d} \eta^{2}}+4 \mu^{2} B_{2} \frac{\mathrm{d} f}{\mathrm{~d} \eta} \frac{\mathrm{d} \bar{g}}{\mathrm{~d} \eta}\right] \mathrm{d} \eta,
\end{aligned}
$$

where

$$
B_{1}+2 B_{2}=H_{3},
$$

and $\bar{g}$ denotes the complex conjugate of $g$. Performing integration by parts on the bilinear form (3.93), we obtain

$$
a(f, g)=\int_{-1}^{1}\left[\mu^{4} H_{2} \frac{\mathrm{d}^{4} f}{\mathrm{~d} \eta^{4}}-2 \mu^{2} H_{3} \frac{\mathrm{d}^{2} f}{\mathrm{~d} \eta^{2}}+H_{1} f\right] \bar{g} \mathrm{~d} \eta .
$$

Thus, the form $a(f, g)$ can alternatively be defined as

$$
a(f, g)=\left(\mathcal{L}_{1}(f), g\right),
$$

where the inner product $(\cdot, \cdot)$ is

$$
(u, v)=\int_{-1}^{1} u \bar{v} \mathrm{~d} \eta
$$


for arbitrary functions $u$ and $v$. The operator $\mathcal{L}_{1}(f)$ is self-adjoint, and the form $a(f, g)$ induces a positive semidefinite norm $a(f, f)$ :

$$
\begin{aligned}
a(f, f)= & \int_{-1}^{1}\left[H_{1}\left\|f-\mu^{2} \nu_{21} \frac{\mathrm{d}^{2} f}{\mathrm{~d} \eta^{2}}\right\|^{2}+\mu^{4} H_{2}\left(1-\nu_{12} \nu_{21}\right)\left\|\frac{\mathrm{d}^{2} f}{\mathrm{~d} \eta^{2}}\right\|^{2}\right. \\
& \left.+4 \mu^{2} B_{2}\left\|\frac{\mathrm{d} f}{\mathrm{~d} \eta}\right\|^{2}\right] \mathrm{d} \eta \geq 0 .
\end{aligned}
$$

This implies that the eigenvalues of $\mathcal{L}_{1}(f)$ are nonnegative. That is,

$$
\lambda \geq 0
$$

for all eigenvalues $\lambda$ of the problem (3.88-3.90), which governs the crosssectional eigenfunctions $f(y)$ and the corresponding eigenvalues of the buckled, travelling orthotropic plate.

\subsubsection{Analytical solution}

The general solutions of the ordinary differential equation (3.88) have the form

$$
f=A e^{p \eta}, \quad p=\frac{\kappa}{\mu},
$$

where $A$ is an arbitrary constant and $\kappa$ is a solution of the following biquadratic algebraic characteristic equation:

$$
H_{2} \kappa^{4}-2 H_{3} \kappa^{2}+\left(H_{1}-\lambda\right)=0 .
$$

The solution can be written as

$$
\kappa_{ \pm}^{2}=\frac{H_{3}}{H_{2}}\left(1 \pm \sqrt{1-\frac{H_{2}\left(H_{1}-\lambda\right)}{H_{3}^{2}}}\right)=\frac{H_{3}}{H_{2}}\left(1 \pm \sqrt{1-\frac{H_{2}(1-\lambda)}{H_{3}^{2}}}\right),
$$

where the upper, and respectively the lower, signs correspond to each other. In the last form on the right, we have used the choice $D_{0}=D_{1}$, which leads to $H_{1}=1$.

Let us consider the range of $\lambda$ where the solution is real-valued. The numbers $\kappa_{ \pm}^{2}$ are real-valued if the expression under the square root in (3.102) is nonnegative. This implies the following lower limit for $\lambda$ :

$$
\lambda_{\mathrm{m}} \equiv 1-\frac{H_{3}^{2}}{H_{2}}<\lambda,
$$

corresponding to a real-valued eigenfunction $f$. Note that in the case $\lambda=\lambda_{\mathrm{m}}$, the solution of (3.101) is 


$$
\kappa= \pm \sqrt{\frac{H_{3}}{H_{2}}},
$$

where both solutions are double roots.

Furthermore, if we require not only $\kappa_{ \pm}^{2}$, but also $\kappa_{ \pm}$to be real-valued, the whole parenthetical expression in (3.102) must then be nonnegative. This gives us an upper limit for $\lambda$ :

$$
\lambda \leq 1 \equiv \lambda_{\max } .
$$

Equation (3.105) holds regardless of the values of the problem parameters.

For the lower limit given by (3.103), it holds that

$$
\lambda_{\mathrm{m}} \leq 0 \quad \text { when } \quad G_{12} \geq G_{\mathrm{H}},
$$

where $G_{12}$ is the in-plane shear modulus of the orthotropic material (which is considered an independent material parameter), and $G_{\mathrm{H}}$ is the geometric average shear modulus. The quantity $G_{\mathrm{H}}$ is given by (2.25) (on p. 29), repeated here for convenience:

$$
G_{\mathrm{H}} \equiv \frac{\sqrt{E_{1} E_{2}}}{2\left(1+\sqrt{\nu_{12} \nu_{21}}\right)} .
$$

By (3.99) and (3.103-3.105), in the case that (3.106) holds, we may seek the lowest eigenvalue in the range $0 \leq \lambda \leq 1$, as was done in the isotropic case in Sect. 3.4.

On the other hand, one can find examples of measurements of $G_{12}$ for paper materials, which indicate $G_{12}<G_{\mathrm{H}}$. See, e.g., the articles of Mann et al (1980), Seo (1999), Yokoyama and Nakai (2007), and Bonnin et al (2000). For such a material,

$$
\lambda_{\mathrm{m}}>0 \quad \text { when } \quad G_{12}<G_{\mathrm{H}} .
$$

This will produce complex solutions $\kappa_{ \pm}$and complex eigenfunctions if $\lambda$ is between zero and $\lambda_{\mathrm{m}}$. In practice however, it has been numerically observed (from (3.115), presented further below) that this interval contains no solutions. Thus, in this case the search for the lowest eigenvalue can be performed in the range $\lambda_{\mathrm{m}} \leq \lambda \leq 1$.

These considerations motivate the definition

$$
\lambda_{\min } \equiv \max \left(\lambda_{\mathrm{m}}, 0\right),
$$

enabling us to define the relevant range for solutions as

$$
\lambda_{\min } \leq \lambda \leq \lambda_{\max }
$$

regardless of the value of the shear modulus $G_{12}$. The quantities $\lambda_{\mathrm{m}}$ and $\lambda_{\max }$ are defined by (3.103) and (3.105), respectively. 
From (3.100) and (3.102) in the case that $\lambda \neq \lambda_{\mathrm{m}}$, we obtain that the general solution can be represented in the form

$$
f(\eta)=A_{1} e^{+\frac{\kappa_{+} \eta}{\mu}}+A_{2} e^{-\frac{\kappa_{+} \eta}{\mu}}+A_{3} e^{+\frac{\kappa_{-} \eta}{\mu}}+A_{4} e^{-\frac{\kappa_{-} \eta}{\mu}}
$$

with unknown constants $A_{1}, A_{2}, A_{3}$ and $A_{4}$.

The eigenvalue boundary value problem $(3.88-3.90)$ is invariant under the symmetry operation $\eta \rightarrow-\eta$, and consequently the eigenforms can be classified into functions that are symmetric $\left(f^{\mathrm{s}}\right)$ or antisymmetric $\left(f^{\mathrm{a}}\right)$ with respect to the origin. Using the relations (3.88-3.90) and (3.111), we obtain a general representation for the function $f^{\mathrm{s}}(\eta)$ and linear algebraic equations for determining the constants $A^{\mathrm{s}}$ and $B^{\mathrm{s}}$ :

$$
\begin{gathered}
f^{\mathrm{s}}(\eta)=A^{\mathrm{s}} \cosh \frac{\kappa_{+} \eta}{\mu}+B^{\mathrm{s}} \cosh \frac{\kappa_{-} \eta}{\mu}, \\
A^{\mathrm{s}}\left(\kappa_{+}^{2}-\beta_{1}\right) \cosh \frac{\kappa_{+}}{\mu}+B^{\mathrm{s}}\left(\kappa_{-}^{2}-\beta_{1}\right) \cosh \frac{\kappa_{-}}{\mu}=0, \\
A^{\mathrm{s}} \kappa_{+}\left(\kappa_{+}^{2}-\beta_{2}\right) \sinh \frac{\kappa_{+}}{\mu}+B^{\mathrm{s}} \kappa_{-}\left(\kappa_{-}^{2}-\beta_{2}\right) \sinh \frac{\kappa_{-}}{\mu}=0,
\end{gathered}
$$

where $A^{\mathrm{s}}$ and $B^{\mathrm{s}}$ are unknown constants. Due to the symmetry (or antisymmetry) of the solution $f$, we have only two independent unknown constants, instead of the four in the general representation (3.111), where the symmetry considerations had not yet been applied.

Proceeding in the same manner as in the isotropic case of Sect. 3.4.2, the conditions for a non-trivial solution to exist in the form of $(3.112-3.114)$ reduce to the requirement that the determinant of the homogeneous linear system (3.113-3.114) vanishes.

Again, at the solution point, the zero determinant condition leads to the linear dependence of the equations $(3.113-3.114)$, providing only one independent condition. Thus, we may solve either of (3.113-3.114) for either $A^{\mathrm{s}}$ or $B^{\mathrm{s}}$, and choose the other (free) coefficient arbitrarily.

After rearrangement, the zero determinant condition can be expressed in the convenient form

$$
\Phi\left(\gamma, \mu, \nu_{12}, E_{1}, E_{2}, G_{12}\right)-\Psi\left(\gamma, \nu_{12}, E_{1}, E_{2}, G_{12}\right)=0,
$$

where

$$
\begin{aligned}
\Phi\left(\gamma, \mu, \nu_{12}, E_{1}, E_{2}, G_{12}\right) & =\tanh \frac{\kappa_{-}}{\mu} \operatorname{coth} \frac{\kappa_{+}}{\mu}, \\
\Psi\left(\gamma, \nu_{12}, E_{1}, E_{2}, G_{12}\right) & =\frac{\kappa_{+}\left(\kappa_{+}^{2}-\beta_{2}\right)\left(\kappa_{-}^{2}-\beta_{1}\right)}{\kappa_{-}\left(\kappa_{+}^{2}-\beta_{1}\right)\left(\kappa_{-}^{2}-\beta_{2}\right)},
\end{aligned}
$$

and 


$$
\kappa_{+}=\kappa_{+}\left(\gamma, \nu_{12}, E_{1}, E_{2}, G_{12}\right), \quad \kappa_{-}=\kappa_{-}\left(\gamma, \nu_{12}, E_{1}, E_{2}, G_{12}\right) .
$$

The obtained transcendental equation (3.115) can be used to determine the eigenvalues

$$
\lambda=\gamma^{2}
$$

corresponding to symmetric eigenfunctions with different values of the parameters $\mu, \nu_{12}, E_{1}, E_{2}$ and $G_{12}$.

In the definitions of $\Phi$ and $\Psi,(3.116-3.117)$, there is no dependence on the parameter $\nu_{21}$, because it depends on $\nu_{12}, E_{1}$ and $E_{2}$ via the compatibility relation (2.36) on p. 31. The independent parameters in $\Phi$ and $\Psi$ can be chosen also in a different way, by choosing any combination of exactly three parameters out of $E_{1}, E_{2}, \nu_{12}$ and $\nu_{21}$. Relation (2.36) can then be used to eliminate the remaining parameter.

Similarly, using the relations (3.89) and (3.90), we can obtain a representation for antisymmetric eigenfunctions $f^{\mathrm{a}}(\eta)$, the equation for determining the corresponding constants $A^{\mathrm{a}}$ and $B^{\mathrm{a}}$, and the transcendental equation

$$
\Phi-\frac{1}{\Psi}=0,
$$

where $\Phi$ and $\Psi$ are the functions defined in (3.116-3.117). These equations can be used for determining the eigenvalues corresponding to antisymmetric eigenforms. The representations differ from (3.112-3.114) through the replacements

$$
\cosh \rightarrow \sinh \quad \text { and } \quad \sinh \rightarrow \cosh .
$$

Again, it turns out that the minimal antisymmetric eigenvalue is higher than the minimal symmetric one, so we will only consider the symmetric case.

In the special case that $\lambda=\lambda_{\mathrm{m}}$, the characteristic equation (3.101) has two double roots (3.104), and then, the general solution has the form

$$
f(\eta)=A_{1} e^{+\frac{\kappa \eta}{\mu}}+A_{2} e^{-\frac{\kappa \eta}{\mu}}+A_{3} \eta e^{+\frac{\kappa \eta}{\mu}}+A_{4} \eta e^{-\frac{\kappa \eta}{\mu}} .
$$

In this case, the symmetric solution has the form

$$
f^{\mathrm{s}}(\eta)=A^{\mathrm{s}} \cosh \frac{\kappa \eta}{\mu}+B^{\mathrm{s}} \eta \sinh \frac{\kappa \eta}{\mu} .
$$

For this solution, we will also have a zero determinant condition (different from (3.115) and (3.120)) but for a fixed $\kappa$. It can be calculated that the determinant condition does not hold for (3.122) with the boundary conditions (3.89-3.90), and thus, there is no symmetric solution of the form (3.122), and we will have no solution when $\lambda=\lambda_{\mathrm{m}}$. The antisymmetric case can be explored in a similar manner.

Similar remarks about finalizing the solution apply as in Sect. 3.4.2. Once (3.115) has been solved, obtaining the minimal symmetric eigenvalue $\gamma_{*}$, the 
corresponding critical velocity of the travelling orthotropic plate can be found from (3.85). The critical velocity is

$$
\left(V_{0}^{\mathrm{div}}\right)^{2}=\frac{T_{0}}{m}+\frac{\gamma_{*}^{2}}{m}\left(\frac{\pi^{2} D_{0}}{\ell^{2}}\right) .
$$

Then, in order to obtain the corresponding eigenmode, we can solve for either $A^{\mathrm{s}}$ or $B^{\mathrm{s}}$, picking either of the equations (3.113-3.114). Recall that the equations are linearly dependent at the solution point $\gamma=\gamma_{*}$, so it does not matter which one is used. The other coefficient (either $B^{\mathrm{s}}$ or $A^{\mathrm{s}}$, respectively) is then the free coefficient of the eigenvalue problem, and can be assigned an arbitrary value. Finally, inserting the obtained $\gamma_{*}, A^{\mathrm{s}}$ and $B^{\mathrm{s}}$ into (3.86), (3.102) and (3.112) gives the eigenmode corresponding to the eigenvalue $\gamma_{*}$.

\subsubsection{Properties of analytical solution}

In this section, we will investigate the properties of the functions $\Phi$ and $\Psi$, when $\lambda$ is in the range $\lambda_{\mathrm{m}} \leq \lambda \leq 1$, where $\lambda_{\mathrm{m}}$ is given by (3.103).

Unlike in the isotropic case described in Sect. 3.4.3, the decoupling between the geometric and material parameters is very minimal. The function $\Psi$ does not depend on the aspect ratio $\mu$ (plate geometry), but both $\Phi$ and $\Psi$ depend on all independent material parameters $\left(\nu_{12}, E_{1}, E_{2}\right.$ and $\left.G_{12}\right)$.

We start our examination by noting that (by direct calculation)

$$
\begin{aligned}
& \Phi\left(\lambda_{\mathrm{m}}\right)=1, \\
& \Psi\left(\lambda_{\mathrm{m}}\right)=1,
\end{aligned}
$$

and

$$
\Phi(1)=0,
$$

regardless of the problem parameters. We defer the evaluation of

$$
\lim _{\lambda \rightarrow \lambda_{\max }} \Psi(\lambda)
$$

to Sect. 3.5.5. Although it is trivial to see that $\Psi$ has a singularity there, because $\kappa_{-} \rightarrow 0^{+}$as $\lambda \rightarrow \lambda_{\max }$, in order to deduce the sign of the singularity we need to know the sign of each of the terms in (3.117).

Let us assume the values of $\nu_{12}, E_{1}, E_{2}$ and $G_{12}$ to be given and that they correspond to some orthotropic material. The qualitative behavior of the functions $\Phi$ and $\Psi$ is illustrated in Fig. 3.6. Recall that the corresponding isotropic case was illustrated further above, Fig. 3.3 in Sect. 3.4.

The range for $\gamma$, which is defined in (3.85), is obtained by taking the square root of each side of the inequality in (3.110). Note that the $x$ axis in Fig. 3.3 
starts at $\gamma_{\min }$. In the isotropic case, we had $\gamma_{\min }=0$, which does not hold in general for the orthotropic case.

Fig. 3.7 shows some examples of $\Phi$ and $\Psi$ plotted for some orthotropic materials. As discussed above, only $\Phi$ depends on the aspect ratio $\ell / 2 b$. We see that the case $G_{12}=G_{\mathrm{H}}$ behaves like the isotropic case, as expected (compare with Sect. 3.4.3). When the value of $G_{12}$ deviates from the geometric average shear modulus (3.107), it is seen that when $G_{12}<G_{\mathrm{H}}$, the curvature of $\Phi$ becomes more pronounced, especially for a large aspect ratio. If $G_{12}>G_{\mathrm{H}}$, the value of both functions at $\gamma=\gamma_{\min }$ decreases (because then $\gamma_{\min }=0>$ $\gamma_{\mathrm{m}}$ ), again especially for a large aspect ratio in the case of $\Phi$.

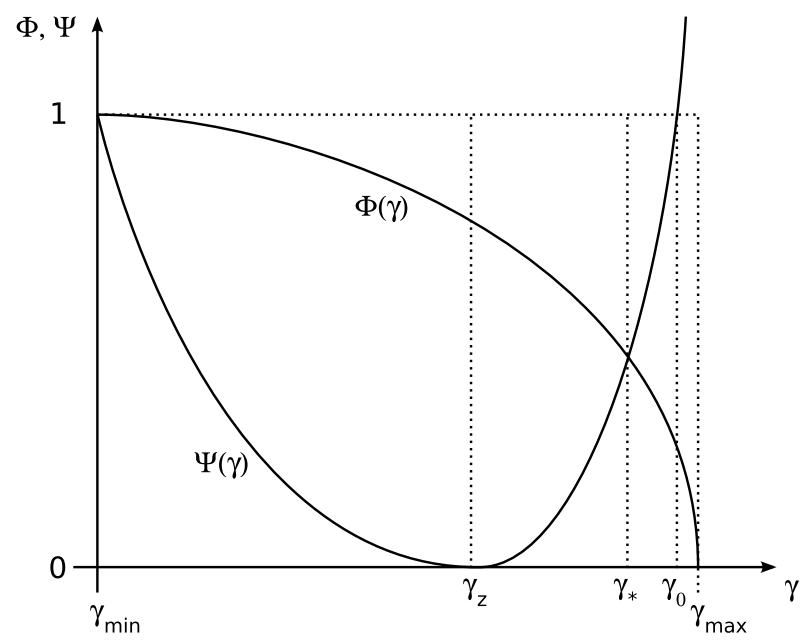

Fig. 3.6 Behaviour of $\Phi$ and $\Psi$ in the orthotropic case, as a function of $\gamma$, when the parameters $D_{1}, D_{2}, D_{3}, \mu, \beta_{1}$ and $\beta_{2}$ are fixed. This is a qualitative drawing illustrating the case $G_{12} \leq G_{\mathrm{H}}$ (for which $\gamma_{\mathrm{min}}=\gamma_{\mathrm{m}}$ ). The main difference between this figure and Fig. 3.3 is that on the $x$ axis, the functions begin at $\gamma_{\min }$ instead of 0 , and the location of the zero of the function $\Psi$ is $\gamma_{\mathrm{z}}$ instead of $1-\nu$. (Reproduced from Banichuk et al 2011a)

When $\gamma$ increases from $\gamma_{\mathrm{m}}$ to $\gamma_{\max }$, the function $\Phi(\gamma, \mu)$ decreases continuously and monotonically from 1 to 0 , i.e.

$$
1 \geq \Phi(\gamma, \mu) \geq 0, \quad \frac{\partial \Phi(\gamma, \mu)}{\partial \gamma}<0, \quad \gamma_{\mathrm{m}} \leq \gamma \leq \gamma_{\max } .
$$

The critical values of the function $\Phi$ in that interval are

$$
\Phi\left(\gamma_{\mathrm{m}}, \mu\right)=\left(\tanh \frac{\kappa-}{\mu} \operatorname{coth} \frac{\kappa_{+}}{\mu}\right)_{\gamma=\gamma_{\mathrm{m}}}=1
$$

and 

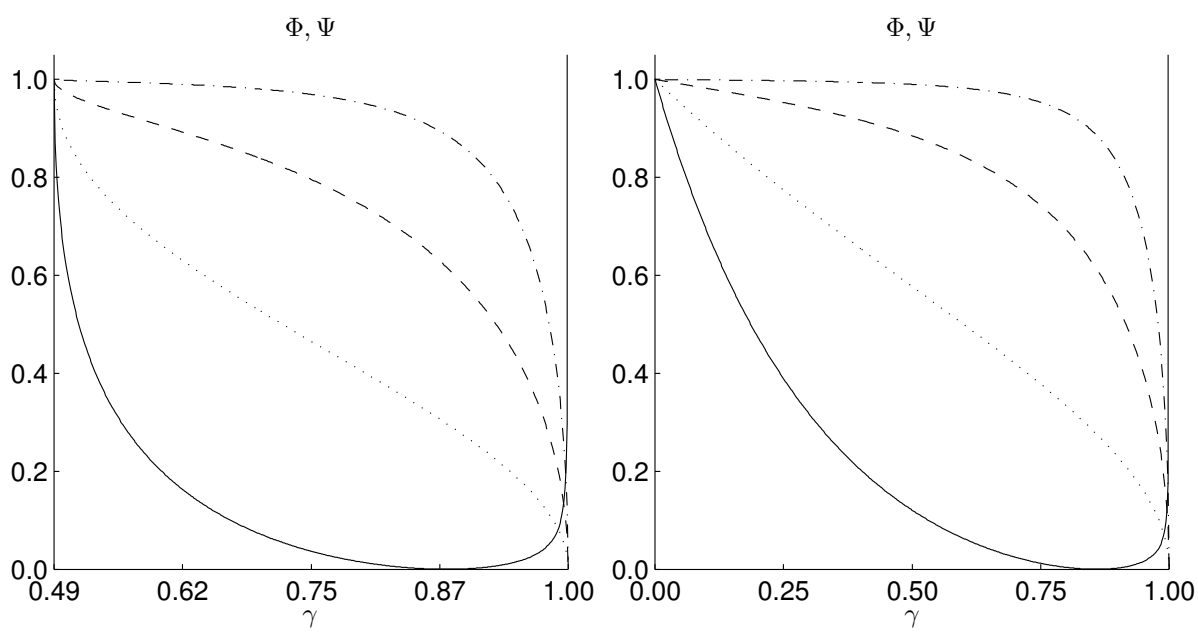

$\Phi, \Psi$
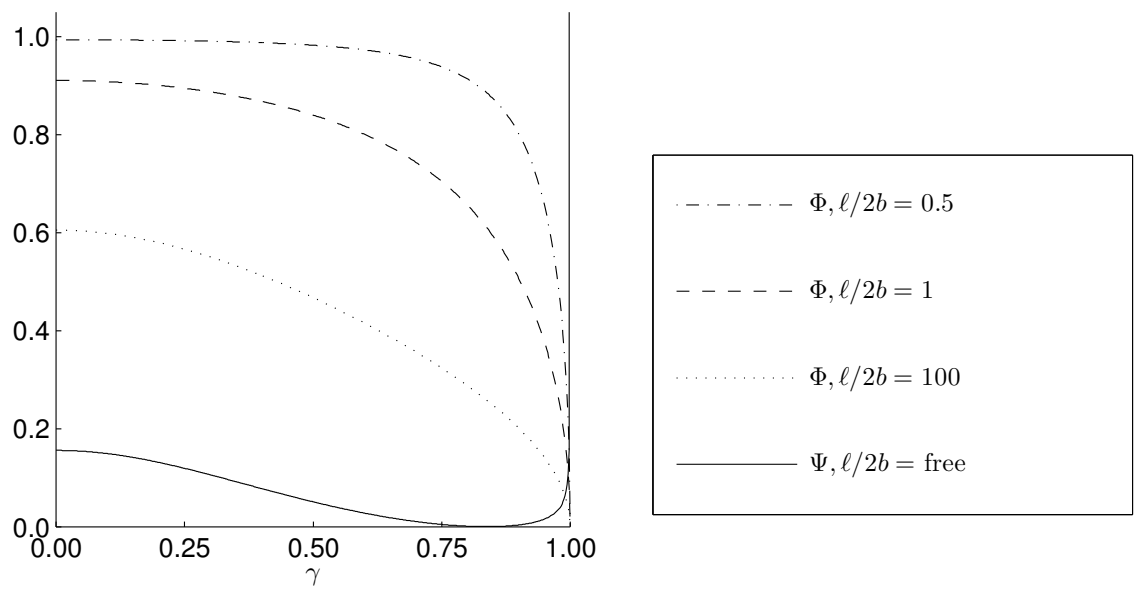

Fig. 3.7 Behaviour of functions $\Phi$ and $\Psi$ for some orthotropic materials, at different aspect ratios $\ell / 2 b$ and different values for the in-plane shear modulus $G_{12}$. For all cases, the other material parameters are $E_{1}=6.8 \mathrm{GPa}, E_{2}=3.4 \mathrm{GPa}$ and $\nu_{12}=0.2, \nu_{21}=0.1$. Note that only $\Phi$ depends on the aspect ratio. Upper left: $G_{12}=0.85 G_{\mathrm{H}}$ (note the scale for $\gamma$ ). Upper right: $G_{12}=G_{\mathrm{H}}$. Lower left: $G_{12}=1.15 G_{\mathrm{H}}$, where $G_{\mathrm{H}}$ is the geometric average shear modulus (3.107). The range of $\gamma$ is $\gamma_{\min } \leq \gamma \leq \gamma_{\max }$, based on (3.85) and (3.110), and evaluated separately for each subfigure. Observe that for the lower right subfigure, $\gamma_{\min }=0>\gamma_{\mathrm{m}}$, causing the qualitative behaviour of the functions to differ from the other cases where $\gamma_{\min }=\gamma_{\mathrm{m}}$. (Reproduced from Banichuk et al 2011a) 


$$
\Phi\left(\gamma_{\max }, \mu\right)=\left(\tanh \frac{\kappa-}{\mu} \operatorname{coth} \frac{\kappa_{+}}{\mu}\right)_{\gamma=\gamma_{\max }}=0 .
$$

The function $\Psi(\gamma)$ decreases monotonically from 1 to 0 in the interval $\gamma_{\mathrm{m}}<$ $\gamma<\gamma_{\mathrm{z}}$ :

$$
1>\Psi(\gamma)>0, \quad \frac{\partial \Psi(\gamma)}{\partial \gamma}<0 .
$$

The values of the function $\Psi$ at the ends of the interval are

$$
\Psi\left(\gamma_{\mathrm{m}}\right)=1
$$

and

$$
\Psi\left(\gamma_{\mathbf{z}}\right)=0 .
$$

The function $\Psi$ increases monotonically in the interval $\gamma_{\mathrm{z}}<\gamma<\gamma_{\max }$, increasing without limit when $\gamma \rightarrow \gamma_{\max }$ :

$$
0<\Psi(\gamma)<\infty, \quad \frac{\partial \Psi(\gamma)}{\partial \gamma}>0
$$

Thus at the ends of the interval, the values of $\Psi$ are

$$
\Psi\left(\gamma_{\mathrm{z}}\right)=0
$$

and

$$
\lim _{\gamma \rightarrow \gamma_{\max }} \Psi(\gamma)=\infty .
$$

The function touches zero at the point

$$
\gamma_{\mathbf{z}}=\sqrt{\beta_{j}^{2} H_{2}-2 \beta_{j} H_{3}+H_{1}},
$$

where $j=1,2$. It will be shown below that $\gamma_{\mathrm{z}}$ is unique. Thus either choice for $j$ results in the same value for $\gamma_{\mathrm{z}}$.

Because

$$
0 \leq \Phi \leq 1 \quad \text { for all } \quad \gamma_{\min } \leq \gamma \leq \gamma_{\max }
$$

the symmetric solution in (3.115) is only possible in the range where $\Psi \leq 1$. Likewise, the antisymmetric solution in (3.120) is only possible in the range where

$$
\frac{1}{\Psi} \leq 1 \quad \text { i.e. } \quad \Psi \geq 1 .
$$

Again, at the point $\gamma=\gamma_{0}$ for which $\Psi=1$, it also holds that $1 / \Psi=1$, and thus at this point we have

$$
\Phi-\Psi=\Phi-\frac{1}{\Psi} \quad \text { at } \quad \gamma=\gamma_{0} .
$$


That is, the functions defined by the left-hand sides of (3.115) and (3.120) will cross at the value $\gamma=\gamma_{0}$.

Equation (3.131), combined with the consideration in the previous paragraph, implies that the eigenvalue $\gamma_{*}$ corresponding to the symmetric solution is always lower than the eigenvalues $\gamma_{1}$ and $\gamma_{2}$ corresponding to the antisymmetric solution. Additionally, since

$$
\Phi\left(\gamma_{\max }, \mu\right)=0 \quad \text { and } \quad \lim _{\gamma \rightarrow \gamma_{\max }} \Psi(\gamma)=\infty,
$$

we see that the second antisymmetric eigenvalue must be $\gamma_{2}=\gamma_{\max }$. For the various values of $\gamma$ defined above, we thus have the ordering

$$
\gamma_{\mathrm{m}} \leq \gamma_{\min }<\gamma_{\mathrm{z}}<\gamma_{*}<\gamma_{0}<\gamma_{1}<\gamma_{2}=\gamma_{\max } .
$$

An analytical expression for $\gamma_{0}$ can be found by using the definitions (3.116-3.117), and solving $\Psi^{2}(\gamma)=1$ for $\gamma$. Again, since we know that $\gamma_{0}>0$, no information is lost by squaring. Let us define the auxiliary expression

$$
\alpha \equiv \sqrt{8 \beta_{1} H_{2} H_{3}+\left({\beta_{1}}^{2}-6 \beta_{1} \beta_{2}+{\beta_{2}}^{2}\right){H_{2}}^{2}},
$$

where the $\beta_{j}$ are the coefficients that appear in the boundary conditions (3.89-3.90). For their expressions in terms of the material parameters, see (3.19).

For the root $\gamma_{0}$ that interests us, the following expression holds:

$$
\gamma_{0}^{2}=\frac{1}{2}\left(\left(\beta_{2}-\beta_{1}\right) \alpha+2 H_{1}-\left({\beta_{1}}^{2}-4 \beta_{1} \beta_{2}+{\beta_{2}}^{2}\right) H_{2}-4 \beta_{1} H_{3}\right) .
$$

Next we will look into some detailed properties of the functions $\Phi$ and $\Psi$, which appear in the minimal symmetric eigenvalue equation for the orthotropic case, (3.115). Then, to finish the orthotropic problem, we will show some numerical examples.

\subsubsection{Analysis of solution properties}

Let us show that the transcendental part $\Phi$ is monotonically decreasing in the open interval $\left(\lambda_{\mathrm{m}}, \lambda_{\max }\right)$. First, we define

$$
g(\lambda) \equiv \sqrt{1-\frac{H_{2}\left(H_{1}-\lambda\right)}{H_{3}^{2}}},
$$


i.e. the square root expression in $\kappa_{ \pm}^{2}$ in (3.102). We see that $g\left(\lambda_{\mathrm{m}}\right)=0$ and $g\left(\lambda_{\max }\right)=1$. Between these extreme values, $g(\lambda)$ increases monotonously as $\lambda$ increases.

We write (3.102) in the form

$$
\kappa_{ \pm}^{2}=\frac{1}{d H_{3}}(1 \pm \sqrt{1-d(1-\lambda)})
$$

where we have defined the auxiliary constant

$$
d \equiv H_{2} / H_{3}^{2}
$$

Differentiating (3.144), we have

$$
\frac{\partial\left(\kappa_{ \pm}^{2}\right)}{\partial \lambda}=\frac{ \pm 1}{2 H_{3} \sqrt{1-d(1-\lambda)}}
$$

where the upper and lower signs correspond to each other. Note that the square root expression in the denominator is $g(\lambda)$ defined by (3.143), and as discussed above, it takes values in the range $(0,1)$ as $\lambda \in\left(\lambda_{\mathrm{m}}, \lambda_{\max }\right)$, and especially, is positive in our range of interest. Thus, (3.145) is always positive for $\kappa_{+}^{2}$ and always negative for $\kappa_{-}^{2}$.

On the other hand, by the rules of differentiation,

$$
\frac{\partial\left(\kappa_{ \pm}^{2}\right)}{\partial \lambda}=2 \kappa_{ \pm} \frac{\partial \kappa_{ \pm}}{\partial \lambda}
$$

and thus

$$
\frac{\partial \kappa_{ \pm}}{\partial \lambda}=\frac{\partial\left(\kappa_{ \pm}^{2}\right)}{\partial \lambda} / 2 \kappa_{ \pm}
$$

Noting that $\kappa_{ \pm}>0$, we can conclude that the signs match:

$$
\operatorname{sign} \frac{\partial \kappa_{ \pm}}{\partial \lambda}=\operatorname{sign} \frac{\partial\left(\kappa_{ \pm}^{2}\right)}{\partial \lambda} .
$$

In the special case of $\lambda=\lambda_{\max }$, we have $\kappa_{-}=0$, rendering the right-hand side of (3.147) singular, but this point is not in our open interval. Now we turn our attention to the transcendental function $\Phi$. Differentiating (3.116) with respect to $\lambda$, we have

$$
\begin{gathered}
\frac{\partial \Phi}{\partial \lambda}=\frac{\partial}{\partial \lambda}\left(\tanh \frac{\kappa_{-}}{\mu}\right) \operatorname{coth} \frac{\kappa_{+}}{\mu}+\left(\tanh \frac{\kappa_{-}}{\mu}\right) \frac{\partial}{\partial \lambda}\left(\operatorname{coth} \frac{\kappa_{+}}{\mu}\right)= \\
\frac{1}{\cosh ^{2} \frac{\kappa_{-}}{\mu}} \cdot \frac{1}{\mu} \cdot \frac{\partial \kappa_{-}}{\partial \lambda} \operatorname{coth} \frac{\kappa_{+}}{\mu}+\tanh \frac{\kappa_{-}}{\mu}\left(-\frac{1}{\sinh ^{2} \frac{\kappa_{+}}{\mu}}\right) \cdot \frac{1}{\mu} \cdot \frac{\partial \kappa_{+}}{\partial \lambda} .
\end{gathered}
$$

In the first term on the right-hand side, 


$$
\frac{\partial \kappa_{-}}{\partial \lambda}<0
$$

by (3.145) and (3.148), while the other factors are all positive, and in the second term,

$$
-\frac{1}{\sinh ^{2} \frac{\kappa_{+}}{\mu}}<0
$$

while all other factors are positive. Thus, both terms on the right side are negative and we conclude that

$$
\frac{\partial \Phi}{\partial \lambda}<0 \text { for all } \lambda \in\left(\lambda_{\mathrm{m}}, \lambda_{\max }\right) .
$$

Consider now the algebraic function $\Psi$. We will show the following properties:

1. The function $\Psi$ has exactly one zero at $\lambda_{z}$.

2. The function $\Psi$ has exactly one singularity, which is located at $\lambda=\lambda_{\max }$, and its sign is positive:

$$
\lim _{\lambda \rightarrow \lambda_{\max }} \Psi(\lambda)=+\infty
$$

3. If the root $\lambda_{\mathrm{z}} \in\left(\lambda_{\mathrm{m}}, \lambda_{\max }\right)$, then the function $\Psi$ is monotonically decreasing in the interval $\lambda \in\left(\lambda_{\mathrm{m}}, \lambda_{\mathrm{z}}\right)$, and monotonically increasing in the interval $\lambda \in\left(\lambda_{\mathrm{z}}, \lambda_{\max }\right)$.

Again, we begin with (3.102). The coefficient in front of the expression can be written as

$$
\frac{H_{3}}{H_{2}}=\frac{D_{3}}{D_{2}}=\nu_{12}+2 \frac{G_{12}}{E_{2}}\left(1-\nu_{12} \nu_{21}\right)
$$

By defining the constants

$$
A \equiv \frac{H_{3}}{H_{2}}=\nu_{12}+2 \frac{G_{12}}{E_{2}}\left(1-\nu_{12} \nu_{21}\right), \quad B \equiv 2 \frac{G_{12}}{E_{2}}\left(1-\nu_{12} \nu_{21}\right),
$$

we see that

$$
\beta_{1}=A-B, \quad \beta_{2}=A+B .
$$

Using (3.154) and (3.143), the definition (3.102) reduces to a more convenient form,

$$
\kappa_{ \pm}^{2}=A(1 \pm g(\lambda)) .
$$

Inserting (3.155) and (3.156) into the definition (3.117), we have

$$
\Psi=\frac{\sqrt{A(1+g(\lambda))}(A g(\lambda)-B)^{2}}{\sqrt{A(1-g(\lambda))}(A g(\lambda)+B)^{2}} .
$$


All factors in the representation (3.157) are always positive, except the second one in the numerator. Thus, the function can only have one zero, which is located at such $\lambda_{\mathrm{z}}$ that

$$
A g\left(\lambda_{z}\right)-B=0 .
$$

The first result is therefore established.

To show the second result, we note that there is exactly one singularity, caused by the first term in the denominator as $g(\lambda) \rightarrow 1$, i.e. as $\lambda \rightarrow \lambda_{\max }$. The function $\Psi$ is continuous outside its singularities. Furthermore, from (3.157), we have that $\Psi \geq 0$ for all $\lambda$ for which the function is nonsingular. Because $\Psi$ is continuous, the singularity must have a positive sign.

To prove the last result we consider the derivative of the function $\Psi$ with regard to $\lambda$. Consider the case where

$$
\lambda_{\mathrm{z}} \in\left(\lambda_{\mathrm{m}}, \lambda_{\max }\right) .
$$

Before we proceed, a motivation of (3.159) may be in order. If $\nu_{12}=0$, by (3.154) we then have $A=B$. Using (3.158), this leads to $g\left(\lambda_{\mathrm{z}}\right)=1$, and further by (3.143), to $\lambda_{\mathrm{z}}=1=\lambda_{\max }$. To see this, observe that $\Psi\left(\lambda_{\max }\right)$ becomes nonsingular if $A=B$, by considering the limit of (3.157) as $g(\lambda) \rightarrow$ $1^{-}$. When $A=B$, the second term in the numerator can be rewritten as $(A g(\lambda)-B)^{2}=(B-A g(\lambda))^{2}=A^{2}(1-g(\lambda))^{2}$ and hence, by cancelling the common factor $\sqrt{A(1-g(\lambda))}$, we are left with $[A(1-g(\lambda))]^{3 / 2}$ in the numerator. Thus, for the special case $A=B$, we have $\Psi\left(\lambda_{\max }\right)=0$.

If the case $\lambda_{\mathrm{z}}=\lambda_{\max }$ is allowed to occur, then by the below argument (which works also for this case almost as-is), we will find that $\Psi$ monotonically decreases in the whole open interval $\lambda \in\left(\lambda_{\mathrm{m}}, \lambda_{\max }\right)$. In such a case, we can no longer be sure that there will exist a point $\lambda=\lambda_{*}$ in $\left(\lambda_{\mathrm{m}}, \lambda_{\max }\right)$ satisfying $\Phi\left(\lambda_{*}\right)=\Psi\left(\lambda_{*}\right)$, since both functions then are monotonically decreasing in the whole open interval. Hence, if $\lambda_{\mathrm{z}}=\lambda_{\max }$ is allowed, we cannot say anything about whether a solution of (3.115) will exist in our interval. Thus, we will limit our consideration to the case $\nu_{12}>0$ (ensuring $A>B$ ), which holds for nearly all naturally occurring materials.

We obtain from (3.157), by direct calculation, that

$$
\begin{aligned}
& \frac{\partial \Psi}{\partial \lambda} \\
& \quad=\frac{\partial g}{\partial \lambda} \frac{(B-A g(\lambda))}{(B+A g(\lambda))^{3}} \frac{\sqrt{A(1-g(\lambda))}}{\sqrt{A(1+g(\lambda))}} \frac{\left(B^{2}-A^{2} g(\lambda)^{2}+4 A B\left(g(\lambda)^{2}-1\right)\right)}{\left(1-g(\lambda)^{2}\right)} .
\end{aligned}
$$

Because all other terms are positive, we have for the sign of the derivative the expression

$$
\operatorname{sign} \frac{\partial \Psi}{\partial \lambda}=\operatorname{sign}\left[(B-A g(\lambda))\left(B^{2}-A^{2} g(\lambda)^{2}+4 A B\left(g(\lambda)^{2}-1\right)\right)\right] .
$$


Because $g(\lambda)$ is monotonically increasing and therefore $\partial g / \partial \lambda>0$, and the zero of the function $\Psi$ is located at $A g\left(\lambda_{z}\right)=B$, we see that

$$
\operatorname{sign}[A g(\lambda)-B]=\operatorname{sign}\left[\lambda-\lambda_{z}\right],
$$

i.e. the sign of the expression $A g(\lambda)-B$ corresponds to whether $\lambda$ is smaller or larger than $\lambda_{z}$.

We can write the expression on the right-hand side of (3.161) as

$$
(B-A g(\lambda))\left[(B-A g(\lambda))(B+A g(\lambda))+4 A B\left(g(\lambda)^{2}-1\right)\right] .
$$

If

$$
B-A g(\lambda)<0 \quad \text { i.e. } \quad \lambda>\lambda_{\mathrm{z}},
$$

the expression in the parentheses at right is negative. The last term is always negative because $g(\lambda)<1$. In this case we have

$$
\left.\frac{\partial \Psi}{\partial \lambda}\right|_{\lambda>\lambda_{z}}>0
$$

The other case

$$
B-A g(\lambda)>0 \quad \text { i.e. } \quad \lambda<\lambda_{z},
$$

is trickier because then the expression in the parentheses at right in (3.163) will have one positive and one negative term. However, we see that the expression represents a parabola with the variable $g(\lambda)$, having zeroes at

$$
g_{0}^{ \pm} \equiv \pm \sqrt{\frac{4 A B-B^{2}}{4 A B-A^{2}}} .
$$

Because $g(\lambda)>0$, we may discard the negative solution $g_{0}^{-}$in (3.167). The expression is negative until $g(\lambda)$ becomes larger than the positive solution $g_{0}^{+}$.

The last question remaining is whether this solution lies within our range. Consider the square root expression on the right-hand side of (3.167). We subtract the denominator from the numerator, looking again at the definitions (3.154), and recall that we required $\nu_{12}>0$ :

$$
\left(4 A B-B^{2}\right)-\left(4 A B-A^{2}\right)=A^{2}-B^{2}>0,
$$

i.e. we find that the numerator is always larger than the denominator. Thus $g_{0}^{+}>1$ and the parabola remains negative in our entire range. The total sign is negative and thus

$$
\left.\frac{\partial \Psi}{\partial \lambda}\right|_{\lambda<\lambda_{z}}<0
$$

which was to be shown. 
We will illustrate the critical divergence velocities and the corresponding buckling modes (divergence modes) of axially moving orthotropic plates by giving some numerical examples. The physical parameters used are varied with the examples. The mass per unit area $m$, the value of homogeneous tension $T_{0}$ and the plate thickness $h$ are kept constant, and the used values for them are given in Table 3.3.

Table 3.3 Physical parameters used in the numerical examples.

\begin{tabular}{lll}
\hline$T_{0}$ & $m$ & $h$ \\
\hline $500 \mathrm{~N} / \mathrm{m}$ & $0.08 \mathrm{~kg} / \mathrm{m}^{2}$ & $10^{-4} \mathrm{~m}$ \\
\hline
\end{tabular}

In Fig. 3.8, slices of buckling modes at $x=\ell / 2$ are presented for four different Young modulus ratios $E_{1} / E_{2}$. We observe that the Young modulus ratio affects the localisation of the buckling mode: the smaller the ratio is, the more the shape is localised near the edges.

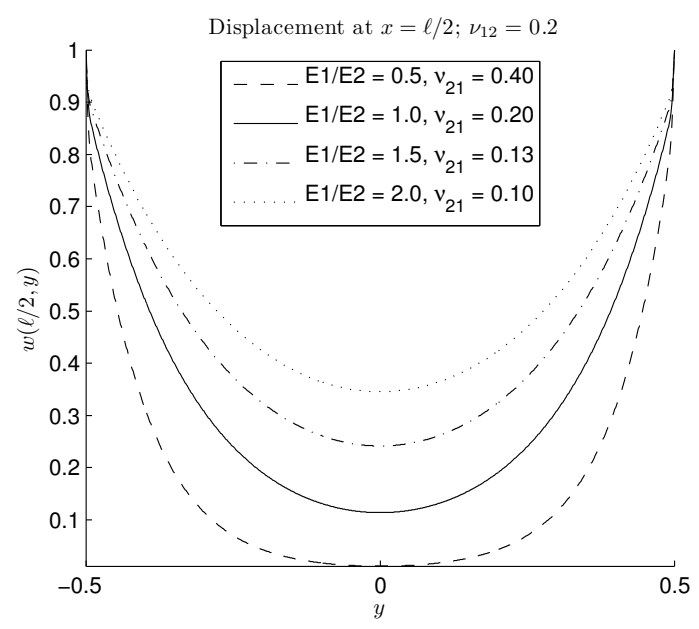

Fig. 3.8 Slices of buckling modes for different Young modulus ratios. Slices of the buckling modes at $x=\ell / 2$ are shown. The ratio between the plate length and the plate width is $\ell /(2 b)=0.01$. The Young modulus in the $x$ direction is $E_{1}=5 \mathrm{GPa}$ and the Poisson ratio $\nu_{12}$ is 0.2 . The Poisson ratio $\nu_{21}$ is calculated from relation (2.36) on page 31. For the shear modulus, the geometric average $G_{\mathrm{H}}$ from (2.25) on page 29 is used. (Reproduced from Banichuk et al 2011a)

The degree of localisation represents the variation of the displacement in the width $(y)$ direction. Relative localisation is high, when most of the displacement occurs near the free edges. The problem parameters affecting the degree of localisation are the aspect ratio $\ell /(2 b)$ (as was seen in Sect. 
3.4.3), the Young modulus ratio $E_{1} / E_{2}$, the Poisson ratio $\nu_{12}$, and the inplane shear modulus $G_{12}$.

In Fig. 3.9, we see three examples of complete buckling shapes for different values of the shear modulus $G_{12}$. The buckling shapes depend significantly on the in-plane shear modulus $G_{12}$. The figure also shows that if the ratio $G_{12} / G_{\mathrm{H}}$ is increased, then the degree of localisation decreases.

In Table 3.4, the values of critical velocities, defined in (3.123), are given for some selected values of the in-plane shear modulus $G_{12}$ and the aspect ratio $\ell /(2 b)$. The row $\ell /(2 b)=0.01$ corresponds to the buckling modes in Fig. 3.9. The effect of the increased in-plane shear modulus is that the value of the critical velocity slightly increases.

Table 3.4 Critical velocities $V_{0}^{\text {div }}(\mathrm{m} / \mathrm{s})$ of an axially moving orthotropic plate for different values of in-plane shear modulus $G_{12}$ and the ratio between the plate length and the plate width $\ell /(2 b) . G_{\mathrm{H}}$ is the geometric average shear modulus, (3.107). The Young moduli are $E_{1}=6.8 \mathrm{GPa}$ and $E_{2}=3.4 \mathrm{GPa}$, and the Poisson ratios $\nu_{12}$ is 0.2 and $\nu_{21}=0.1$.

\begin{tabular}{llll}
\hline & $G_{12}$ & & \\
\cline { 2 - 4 }$\ell /(2 b)$ & $0.7 G_{\mathrm{H}} \approx 1.47 \mathrm{GPa}$ & $G_{\mathrm{H}} \approx 2.11 \mathrm{GPa}$ & $1.3 G_{\mathrm{H}} \approx 2.74 \mathrm{GPa}$ \\
\hline 0.01 & $83.4456 \mathrm{~m} / \mathrm{s}$ & $83.4461 \mathrm{~m} / \mathrm{s}$ & $83.4463 \mathrm{~m} / \mathrm{s}$ \\
0.1 & $79.1020 \mathrm{~m} / \mathrm{s}$ & $79.1020 \mathrm{~m} / \mathrm{s}$ & $79.1020 \mathrm{~m} / \mathrm{s}$ \\
1 & $79.0574 \mathrm{~m} / \mathrm{s}$ & $79.0574 \mathrm{~m} / \mathrm{s}$ & $79.0574 \mathrm{~m} / \mathrm{s}$ \\
\hline
\end{tabular}


$\ell / 2 b=0.01, E_{1} / E_{2}=2, \nu_{12}=0.2, \nu_{21}=0.1, G_{12}=1.47 \mathrm{GPa}$

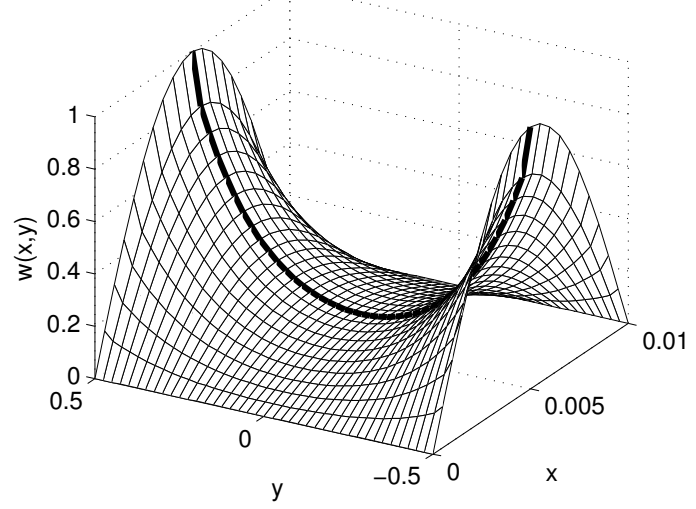

$\ell / 2 b=0.01, E_{1} / E_{2}=2, \nu_{12}=0.2, \nu_{21}=0.1, G_{12}=G_{H}$

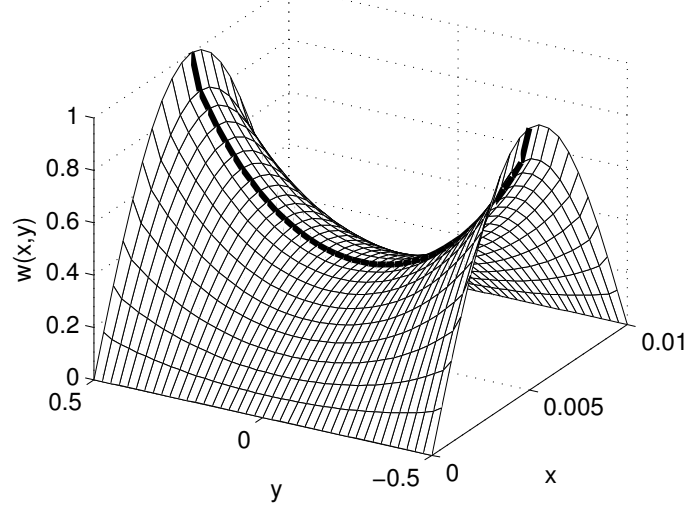

$\ell / 2 b=0.01, E_{1} / E_{2}=2, \nu_{12}=0.2, \nu_{21}=0.1, G_{12}=2.74 \mathrm{GPa}$

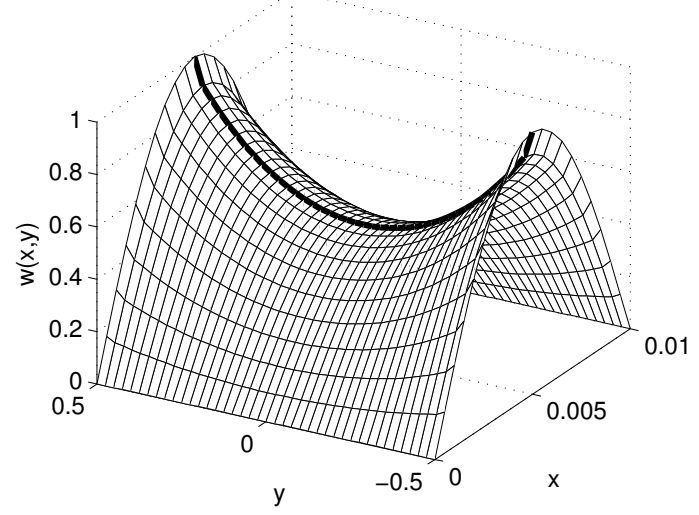

Fig. 3.9 Buckling modes for three different in-plane shear moduli. The ratio between the plate length and the plate width is $\ell /(2 b)=0.01$. Top: $G_{12}=0.7 G_{\mathrm{H}} ;$ Middle: $G_{12}=G_{\mathrm{H}}$; Bottom: $G_{12}=1.3 G_{\mathrm{H}}$, where $G_{12}$ is the in-plane shear modulus and $G_{\mathrm{H}}$ is the geometric average shear modulus, (3.107). The Young moduli are $E_{1}=6.8 \mathrm{GPa}$ and $E_{2}=3.4 \mathrm{GPa}$, and the Poisson ratio $\nu_{12}$ is 0.2 . The Poisson ratio $\nu_{21}$ is calculated from relation (2.36) on page 31 , leading to $\nu_{21}=0.1$. (Reproduced from Banichuk et al 2011a) 


\section{References}

Alava M, Niskanen K (2006) The physics of paper. Reports on Progress in Physics 69(3):669-723

Archibald FR, Emslie AG (1958) The vibration of a string having a uniform motion along its length. ASME Journal of Applied Mechanics 25:347-348

Banichuk N, Jeronen J, Neittaanmäki P, Tuovinen T (2010a) On the instability of an axially moving elastic plate. International Journal of Solids and Structures 47(1):91-99, URL http://dx.doi.org/10.1016/j.ijsolstr.2009.09.020

Banichuk N, Jeronen J, Neittaanmäki P, Tuovinen T (2010b) Static instability analysis for travelling membranes and plates interacting with axially moving ideal fluid. Journal of Fluids and Structures 26(2):274-291, URL http://dx.doi.org/10.1016/j.jfluidstructs.2009.09.006

Banichuk N, Jeronen J, Kurki M, Neittaanmäki P, Saksa T, Tuovinen $\mathrm{T}$ (2011a) On the limit velocity and buckling phenomena of axially moving orthotropic membranes and plates. International Journal of Solids and Structures 48(13):2015-2025, URL http://dx.doi.org/10.1016/j.ijsolstr.2011.03.010

Banichuk N, Jeronen J, Neittaanmäki P, Tuovinen T (2011b) Dynamic behaviour of an axially moving plate undergoing small cylindrical deformation submerged in axially flowing ideal fluid. Journal of Fluids and Structures 27(7):986-1005, URL http://dx.doi.org/10.1016/j.jfluidstructs.2011.07.004

Banichuk N, Jeronen J, Neittaanmäki P, Saksa T, Tuovinen T (2013) Theoretical study on travelling web dynamics and instability under non-homogeneous tension. International Journal of Mechanical Sciences 66C:132-140, URL http://dx.doi.org/10.1016/j.ijmecsci.2012.10.014

Biancolini ME, Brutti C, Reccia L (2005) Approximate solution for free vibrations of thin orthotropic rectangular plates. Journal of Sound and Vibration 288(1-2):321 - 344, DOI: 10.1016/j.jsv.2005.01.005

Bolotin VV (1963) Nonconservative Problems of the Theory of Elastic Stability. Pergamon Press, New York

Bonnin A, Huchon R, Deschamps M (2000) Ultrasonic waves propagation in absorbing thin plates: Application to paper characterization. Ultrasonics 37(8):555-563, URL http://dx.doi.org/10.1016/S0041-624X(99)00106-7

Chang YB, Moretti PM (1991) Interaction of fluttering webs with surrounding air. TAPPI Journal 74(3):231-236

Chen G, Coleman MP, Ding Z (1998) Some corner effects on the loss of selfadjointness and the non-excitation of vibration for thin plates and shells. The Quarterly Journal of Mechanics and Applied Mathematics 51(2):213240, DOI:10.1093/qjmam/51.2.213

Chen LQ, Ding H (2010) Steady-state transverse response in coupled planar vibration of axially moving viscoelastic beams. ASME Journal of Vibrations and Acoustics 132:011,009-1-9, http://dx.doi.org/10.1115/1.4000468 
Chen LQ, Wang B (2009) Stability of axially accelerating viscoelastic beams: asymptotic perturbation analysis and differential quadrature validation. European Journal of Mechanics - A/Solids 28(4):786 - 791, DOI: $10.1016 /$ j.euromechsol.2008.12.002

Chen LQ, Zhao WJ (2005) A numerical method for simulating transverse vibrations of an axially moving string. Applied Mathematics and Computation 160(2):411 - 422, DOI: 10.1016/j.amc.2003.11.012

Chen LQ, Chen H, Lim C (2008) Asymptotic analysis of axially accelerating viscoelastic strings. International Journal of Engineering Science 46(10):976 - 985, DOI: 10.1016/j.ijengsci.2008.03.009

Chonan S (1986) Steady state response of an axially moving strip subjected to a stationary lateral load. Journal of Sound and Vibration 107:155-165

Ding H, Chen LQ (2008) Stability of axially accelerating viscoelastic beams: multi-scale analysis with numerical confirmations. European Journal of Mechanics - A/Solids 27(6):1108 - 1120, DOI: 10.1016/j.euromechsol.2007.11.014

Erkkilä AL, Leppänen T, Hämäläinen J (2013) Empirical plasticity models applied for paper sheets having different anisotropy and dry solids content levels. Int $\mathrm{J}$ Solids Struct in press, URL http://dx.doi.org/10.1016/j.ijsolstr.2013.03.004

Euler L (1766) De motu vibratorio tympanorum. Novi Commentarii academiae scientiarum imperialis Petropolitanae 10:243-260, URL http://eulerarchive.maa.org/pages/E302.html

Frondelius T, Koivurova H, Pramila A (2006) Interaction of an axially moving band and surrounding fluid by boundary layer theory. Journal of Fluids and Structures 22(8):1047-1056

Fung RF, Huang JS, Chen YC (1997) The transient amplitude of the viscoelastic travelling string: An integral constitutive law. Journal of Sound and Vibration 201(2):153 - 167, DOI: 10.1006/jsvi.1996.0776

Fung RF, Huang JS, Chen YC, Yao CM (1998) Nonlinear dynamic analysis of the viscoelastic string with a harmonically varying transport speed. Computers \& Structures 66(6):777 - 784, DOI: 10.1016/S0045-7949(98)00001-7

Gorman DJ (1982) Free Vibration Analysis of Rectangular Plates. Elsevier North Holland, Inc., ISBN 0-444-00601-X

Göttsching L, Baumgarten HL (1976) Triaxial deformation of paper under tensile load. In: The Fundamental Properties of Paper Related to Its Uses, Vol. 1, Technical Division of the British Paper and Board Industry Federation, pp 227-252

Hatami S, Azhari M, Saadatpour MM, Memarzadeh P (2009) Exact free vibration of webs moving axially at high speed. In: AMATH'09: Proceedings of the 15th American Conference on Applied Mathematics, World Scientific and Engineering Academy and Society (WSEAS), Stevens Point, Wisconsin, USA, pp 134-139, houston, USA 
Hosaka H, Crandall SH (1992) Self-excited vibrations of a flexible disk rotating on an air film above a flat surface. Acta Mechanica 3:115-127, supplement

Jeronen J (2011) On the mechanical stability and out-of-plane dynamics of a travelling panel submerged in axially flowing ideal fluid: a study into paper production in mathematical terms. PhD thesis, Department of Mathematical Information Technology, University of Jyväskylä, URL http://julkaisut.jyu.fi/?id=978-951-39-4596-1, Jyväskylä studies in computing 148. ISBN 978-951-39-4595-4 (book), ISBN 978-951-39-4596-1 (PDF)

Kong L, Parker RG (2004) Approximate eigensolutions of axially moving beams with small flexural stiffness. Journal of Sound and Vibration 276:459-469

Kshirsagar S, Bhaskar K (2008) Accurate and elegant free vibration and buckling studies of orthotropic rectangular plates using untruncated infinite series. Journal of Sound and Vibration 314(3-5):837 - 850, DOI: 10.1016/j.jsv.2008.01.013

Kulachenko A, Gradin P, Koivurova H (2007a) Modelling the dynamical behaviour of a paper web. Part I. Computers \& Structures 85:131-147

Kulachenko A, Gradin P, Koivurova H (2007b) Modelling the dynamical behaviour of a paper web. Part II. Computers \& Structures 85:148-157

Kurki M, Lehtinen A (2009) In-plane strain field theory for 2-d moving viscoelastic webs. In: Papermaking Research Symposium 2009 (Kuopio, Finland), PRS

Lee U, Oh H (2005) Dynamics of an axially moving viscoelastic beam subject to axial tension. International Journal of Solids and Structures 42(8):2381 - 2398, URL http://dx.doi.org/10.1016/j.ijsolstr.2004.09.026

Lin CC (1997) Stability and vibration characteristics of axially moving plates. International Journal of Solids and Structures 34(24):3179-3190

Lin CC, Mote CD (1995) Equilibrium displacement and stress distribution in a two-dimensional, axially moving web under transverse loading. ASME Journal of Applied Mechanics 62:772-779

Lin CC, Mote CD (1996) Eigenvalue solutions predicting the wrinkling of rectangular webs under non-linearly distributed edge loading. Journal of Sound and Vibration 197(2):179-189

Mann RW, Baum GA, Habeger CC (1980) Determination of all nine orthotropic elastic constants for machine-made paper. TAPPI Journal 63(2):163-166

Marynowski K (2008) Dynamics of the Axially Moving Orthotropic Web, Lecture Notes in Applied and Computational Mechanics, vol 38. SpringerVerlag, Germany

Marynowski K, Kapitaniak T (2002) Kelvin-Voigt versus Bürgers internal damping in modeling of axially moving viscoelastic web. International Journal of Non-Linear Mechanics 37(7):1147 - 1161, DOI: 10.1016/S00207462(01)00142-1 
Miranker WL (1960) The wave equation in a medium in motion. IBM Journal of Research and Development 4:36-42

Mockensturm EM, Guo J (2005) Nonlinear vibration of parametrically excited, viscoelastic, axially moving strings. ASME Journal of Applied Mechanics 72(3):374-380, DOI: $10.1115 / 1.1827248$

Mote CD (1972) Dynamic stability of axially moving materials. Shock and Vibration Digest 4(4):2-11

Mote CD Jr, Wickert JA (1991) Response and discretization methods for axially moving materials. Applied Mechanics Review 44(11):S279-S284

Mujumdar AS, Douglas WJM (1976) Analytical modelling of sheet flutter. Svensk Papperstidning 79:187-192

Niemi J, Pramila A (1986) Vibration analysis of an axially moving membrane immersed into ideal fluid by FEM. Tech. rep., Tampereen teknillinen korkeakoulu (Tampere University of Technology), Tampere

Oh H, Cho J, Lee U (2004) Spectral element analysis for an axially moving viscoelastic beam. Journal of Mechanical Science and Technology 18(7):1159-1168, URL http://dx.doi.org/10.1007/BF02983290, DOI: $10.1007 /$ BF 02983290

Païdoussis MP (1998) Fluid-Structure Interactions: Slender Structures and Axial Flow, vol 1. Academic Press, ISBN 0-12-544360-9

Païdoussis MP (2004) Fluid-Structure Interactions: Slender Structures and Axial Flow, vol 2. Elsevier Academic Press, ISBN 0-12-544361-7

Païdoussis MP (2005) Some unresolved issues in fluid-structure interactions. Journal of Fluids and Structures 20(6):871-890

Païdoussis MP (2008) The canonical problem of the fluid-conveying pipe and radiation of the knowledge gained to other dynamics problems across applied mechanics. Journal of Sound and Vibration 310:462-492

Parker RG (1998) On the eigenvalues and critical speed stability of gyroscopic continua. ASME Journal of Applied Mechanics 65:134-140

Pramila A (1986) Sheet flutter and the interaction between sheet and air. TAPPI Journal 69(7):70-74

Russo L (2004) The Forgotten Revolution: How Science Was Born in 300 BC and Why it Had to Be Reborn. Springer, ISBN 978-3540203964

Sack RA (1954) Transverse oscillations in traveling strings. British Journal of Applied Physics 5:224-226

Seo YB (1999) Determination of in-plane shear properties by an off-axis tension method and laser speckle photography. Journal of Pulp and Paper Sciences 25(9):321-325

Shen JY, Sharpe L, McGinley WM (1995) Identification of dynamic properties of plate-like structures by using a continuum model. Mechanics Research Communications 22(1):67-78

Shin C, Chung J, Kim W (2005) Dynamic characteristics of the out-of-plane vibration for an axially moving membrane. Journal of Sound and Vibration 286(4-5):1019-1031 
Simpson A (1973) Transverse modes and frequencies of beams translating between fixed end supports. Journal of Mechanical Engineering Science 15:159-164

Skowronski J, Robertson AA (1985) A phenomenological study of the tensile deformation properties of paper. Journal of Pulp and Paper Sciences 11(1):J21-J28

Skutch R (1897) Uber die Bewegung eines gespannten Fadens, weicher gezwungen ist durch zwei feste Punkte, mit einer constanten Geschwindigkeit zu gehen, und zwischen denselben in Transversalschwingungen von gerlinger Amplitude versetzt wird. Annalen der Physik und Chemie 61:190-195

Swope RD, Ames WF (1963) Vibrations of a moving threadline. Journal of the Franklin Institute 275:36-55

Sygulski R (2007) Stability of membrane in low subsonic flow. International Journal of Non-Linear Mechanics 42(1):196-202

Thorpe JL (1981) Paper as an orthotropic thin plate. TAPPI Journal 64(3):119-121

Timoshenko SP, Woinowsky-Krieger S (1959) Theory of plates and shells, 2nd edn. New York : Tokyo : McGraw-Hill, ISBN 0-07-085820-9

Tuovinen T (2011) Analysis of stability of axially moving orthotropic membranes and plates with linear non-homogeneous tension profile. PhD thesis, Department of Mathematical Information Technology, University of Jyväskylä, URL http://julkaisut.jyu.fi/?id=978-951-39-4578-7, Jyväskylä studies in computing 147. ISBN 978-951-39-4577-0 (book), ISBN 978-95139-4578-7 (PDF)

Wang X (2003) Instability analysis of some fluid-structure interaction problems. Computers \& Fluids 32(1):121-138

Wang Y, Huang L, Liu X (2005) Eigenvalue and stability analysis for transverse vibrations of axially moving strings based on Hamiltonian dynamics. Acta Mechanica Sinica 21:485-494

Wickert JA (1992) Non-linear vibration of a traveling tensioned beam. International Journal of Non-Linear Mechanics 27(3):503-517

Wickert JA, Mote CD (1990) Classical vibration analysis of axially moving continua. ASME Journal of Applied Mechanics 57:738-744

Xing Y, Liu B (2009) New exact solutions for free vibrations of rectangular thin plates by symplectic dual method. Acta Mechanica Sinica 25:265-270

Yang XD, Zhang W, Chen LQ, Yao MH (2012) Dynamical analysis of axially moving plate by finite difference method. Nonlinear Dynamics 67(2):9971006, URL http://dx.doi.org/10.1007/s11071-011-0042-2

Yokoyama T, Nakai K (2007) Evaluation of in-plane orthotropic elastic constants of paper and paperboard. In: 2007 SEM Annual Conference \& Exposition on Experimental and Applied Mechanics 


\title{
Chapter 4 Non-homogeneous tension profile
}

\begin{abstract}
In this chapter, we will look at the influence of a skewed tension profile on the divergence instability of a travelling, thin elastic plate. The travelling plate is subjected to axial tension at the supports, but the tension distribution along the supports is not uniform. For the nonuniformity, we will use a linear distribution.

First, we will perform a dynamic analysis of small time-harmonic vibrations, after which we will concentrate on the divergence instability problem. We will see that a small inhomogeneity in the applied tension may have a large effect on the divergence modes, and that inhomogeneity in the tension profile may significantly decrease the critical velocity of the plate.
\end{abstract}

\subsection{Dynamic analysis of axially moving plates}

Let a rectangular part of the plate

$$
\Omega \equiv\left\{(x, y) \in \mathbb{R}^{2} \mid 0<x<\ell,-b<y<b\right\}
$$

be travelling at a constant velocity $V_{0}$ in the $x$ direction between two rollers located at $x=0$ and $x=\ell$, where $\ell$ and $b$ are prescribed parameters. See Fig. 4.1. Let the considered part of the band be represented as an isotropic elastic plate, having constant thickness $h$, Poisson ratio $\nu$, Young modulus $E$ and bending rigidity $D$. We will make some notes on the orthotropic case later.

The plate is subjected to in-plane distributed forces

$$
g=g(y)=T_{0}+T(y)
$$

applied at the plate boundaries $x=0$ and $x=\ell$, acting in the $x$ direction. The constant $T_{0}>0$ and the function $T(y)$, characterizing non-homogeneous 


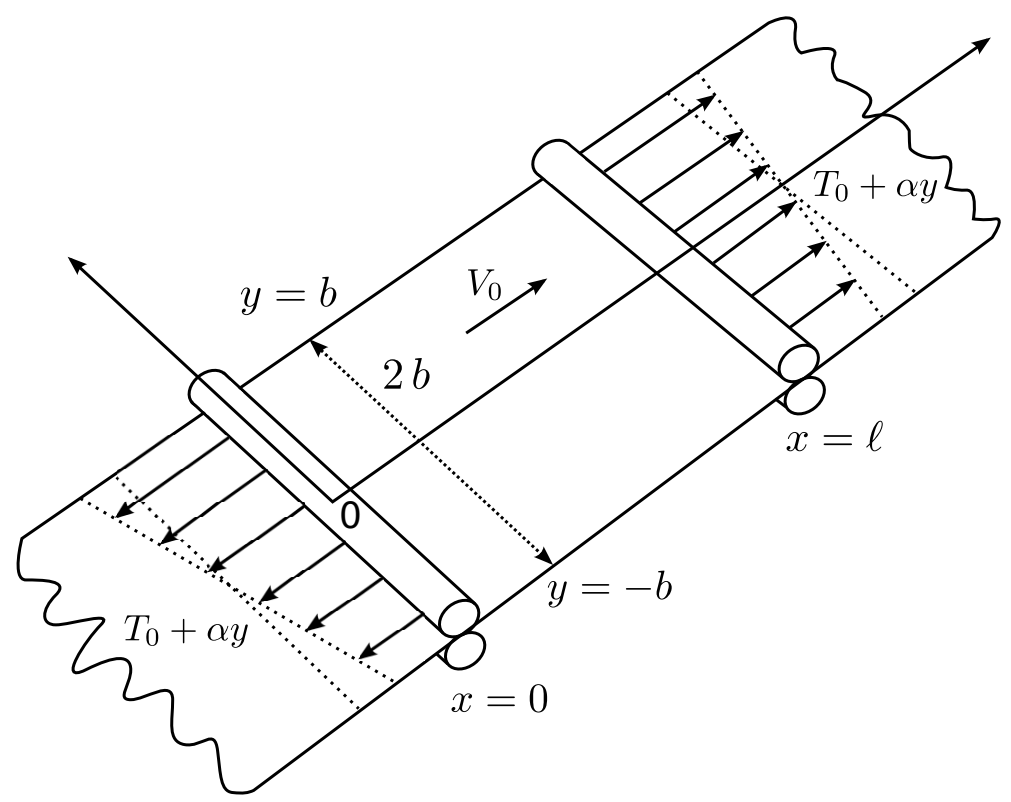

Fig. 4.1 Problem setup. A plate travelling at a constant velocity $V_{0}$ between two rollers placed at $x=0$ and $x=\ell$. The tension profile is non-homogeneous and the tension is positive everywhere. (Reproduced from Banichuk et al 2013)

in-plane tension of the axially moving plate, are considered given. The sides of the plate $\{x=0,-b \leq y \leq b\}$ and $\{x=\ell,-b \leq y \leq b\}$ are simply supported, and the sides $\{y=-b, 0 \leq x \leq \ell\}$ and $\{y=b, 0 \leq x \leq \ell\}$ are free of tractions.

\subsection{Transverse vibrations}

The transverse displacement (out-of-plane deflection) of the travelling plate is described by the deflection function $w$, which depends on the space coordinates $x$ and $y$, and time $t$. The differential equation for small transverse vibrations has the form

$$
m\left(\frac{\partial^{2} w}{\partial t^{2}}+2 V_{0} \frac{\partial^{2} w}{\partial x \partial t}+V_{0}^{2} \frac{\partial^{2} w}{\partial x^{2}}\right)=\mathcal{L}^{\mathrm{M}}(w)-\mathcal{L}^{\mathrm{B}}(w), \quad \text { in } \Omega .
$$

The left-hand side in (4.2) contains three terms, respectively representing a local acceleration, a Coriolis acceleration and a centripetal acceleration. The membrane operator $\mathcal{L}^{\mathrm{M}}$ on the right-hand side of equation (4.2) is 


$$
\mathcal{L}^{\mathrm{M}}(w)=T_{x x} \frac{\partial^{2} w}{\partial x^{2}}+2 T_{x y} \frac{\partial^{2} w}{\partial x \partial y}+T_{y y} \frac{\partial^{2} w}{\partial y^{2}} .
$$

The coefficients $T_{x x}, T_{x y}, T_{y y}$ of the linear operator $\mathcal{L}^{\mathrm{M}}$ are related to the corresponding in-plane stresses $\sigma_{x x}, \sigma_{x y}$ and $\sigma_{y y}$ by the expressions

$$
T_{i j}=h \sigma_{i j}
$$

The linear bending operator $\mathcal{L}^{\mathrm{B}}$ is given by the expression

$$
\mathcal{L}^{\mathrm{B}}(w)=D \Delta^{2} w=D\left(\frac{\partial^{4} w}{\partial x^{4}}+2 \frac{\partial^{4} w}{\partial x^{2} \partial y^{2}}+\frac{\partial^{4} w}{\partial y^{4}}\right)
$$

in the case of an isotropic elastic plate.

Boundary conditions for the deflection function $w$, corresponding to the simply supported boundaries and the free boundaries, can be written in the following form (see, e.g., Timoshenko and Woinowsky-Krieger 1959)

$$
\begin{gathered}
(w)_{x=0, \ell}=0, \quad\left(\frac{\partial^{2} w}{\partial x^{2}}\right)_{x=0, \ell}=0, \quad-b \leq y \leq b, \\
\left(\frac{\partial^{2} w}{\partial y^{2}}+\nu \frac{\partial^{2} w}{\partial x^{2}}\right)_{y= \pm b}=0, \quad 0 \leq x \leq \ell, \\
\left(\frac{\partial^{3} w}{\partial y^{3}}+(2-\nu) \frac{\partial^{3} w}{\partial x^{2} \partial y}\right)_{y= \pm b}=0, \quad 0 \leq x \leq \ell .
\end{gathered}
$$

We represent the in-plane tensions $T_{x x}, T_{x y}$ and $T_{y y}$ with the help of the Airy stress function $\Upsilon$ :

$$
T_{x x}=\frac{\partial^{2} \Upsilon}{\partial y^{2}}, \quad T_{y y}=\frac{\partial^{2} \Upsilon}{\partial x^{2}}, \quad T_{x y}=-\frac{\partial^{2} \Upsilon}{\partial x \partial y} .
$$

In this case of an isotropic plate, the Airy stress function $\Upsilon$ satisfies the biharmonic equation (see (2.46) of Sect. 2.2)

$$
\Delta^{2} \Upsilon \equiv \frac{\partial^{4} \Upsilon}{\partial x^{4}}+2 \frac{\partial^{4} \Upsilon}{\partial x^{2} \partial y^{2}}+\frac{\partial^{4} \Upsilon}{\partial y^{4}}=0
$$

In what follows, we will concentrate on a linear tension distribution. The boundary conditions for the tension are (2.29) and (2.30) of Sect. 2.2, repeated here for convenience:

$$
\begin{gathered}
T_{x x}=g(y), \quad T_{x y}=0 \quad \text { at } x=0, \ell, \quad-b \leq y \leq b, \\
T_{y y}=0, \quad T_{x y}=0 \quad \text { at } y= \pm b, \quad 0 \leq x \leq \ell .
\end{gathered}
$$


The boundary conditions satisfied by $\Upsilon$, corresponding to (2.29) and (2.30) are

$$
\begin{gathered}
\left(\frac{\partial^{2} \Upsilon}{\partial y^{2}}\right)_{x=0, \ell}=g(y), \quad\left(\frac{\partial^{2} \Upsilon}{\partial x \partial y}\right)_{x=0, \ell}=0, \quad-b \leq y \leq b \\
\left(\frac{\partial^{2} \Upsilon}{\partial x^{2}}\right)_{y= \pm b}=0, \quad\left(\frac{\partial^{2} \Upsilon}{\partial x \partial y}\right)_{y= \pm b}=0, \quad 0 \leq x \leq \ell .
\end{gathered}
$$

Recall that the tensions expressed via the stress function $\Upsilon$ in (4.8) will satisfy the equilibrium of in-plane tensions for any function $\Upsilon$ that is smooth enough. The equilibrium equations are (2.28) of Sect. 2.2, repeated here for convenience:

$$
\frac{\partial T_{x x}}{\partial x}+\frac{\partial T_{x y}}{\partial y}=0, \quad \frac{\partial T_{x y}}{\partial x}+\frac{\partial T_{y y}}{\partial y}=0 .
$$

Equation (4.9), which must be solved, expresses the condition of compatibility for the tensions.

In what follows, we will concentrate on a linear tension distribution, and use the rigorous solution of the boundary value problem (4.9-4.11) corresponding to the case that

$$
g(y)=T_{0}+\alpha y \equiv T_{0}+T(y) .
$$

Here $\alpha>0$ is a given constant that will be called the tension profile skew parameter. We have

$$
\Upsilon(x, y)=T_{0} \frac{y^{2}}{2}+\alpha \frac{y^{3}}{6}+c_{1} x+c_{2} y+c_{0}, \quad(x, y) \in \Omega .
$$

Here $c_{0}, c_{1}$ and $c_{2}$ are arbitrary constants. The corresponding tensions will be

$$
T_{x x}(x, y)=T_{0}+\alpha y, \quad T_{x y}(x, y)=0, \quad T_{y y}(x, y)=0, \quad(x, y) \in \Omega .
$$

In this case, the dynamic equation takes the form

$$
\begin{gathered}
\frac{\partial^{2} w}{\partial t^{2}}+2 V_{0} \frac{\partial^{2} w}{\partial x \partial t}+\left(V_{0}^{2}-C^{2}\right) \frac{\partial^{2} w}{\partial x^{2}}-\frac{T(y)}{m} \frac{\partial^{2} w}{\partial x^{2}} \\
+\frac{D}{m}\left(\frac{\partial^{4} w}{\partial x^{4}}+2 \frac{\partial^{4} w}{\partial x^{2} \partial y^{2}}+\frac{\partial^{4} w}{\partial y^{4}}\right)=0, \quad(x, y) \in \Omega,
\end{gathered}
$$

where

$$
C=\sqrt{\frac{T_{0}}{m}}, \quad \text { and } \quad T(y)=\alpha y .
$$


Following the approach of Bolotin (1963), let us represent the solution of the nonstationary boundary value problem for the partial differential equation (4.15) with the boundary conditions (4.5-4.7) using the time-harmonic trial function

$$
w(x, y, t)=W(x, y) e^{s t}, \quad s=i \omega .
$$

Here, $\omega$ is the frequency of the small transverse vibrations, and $s$ is the stability exponent, which is a complex number. As was explained in Chap. 3.2 , if $s$ is purely imaginary, then the plate performs harmonic vibrations with a small amplitude, and its motion can be considered to be stable. If the real part of $s$ becomes positive, then the transverse vibrations grow exponentially and, consequently, the behaviour of the plate is unstable. Using this (complexvalued) representation we will have

$$
s^{2} W+2 s V_{0} \frac{\partial W}{\partial x}+\left(V_{0}^{2}-C^{2}\right) \frac{\partial^{2} W}{\partial x^{2}}-\frac{T(y)}{m} \frac{\partial^{2} W}{\partial x^{2}}+\frac{D}{m} \triangle^{2} W=0 .
$$

The boundary conditions for $W$ follow from (4.5-4.7), by inserting (4.16). We obtain

$$
\begin{gathered}
(W)_{x=0, \ell}=0, \quad\left(\frac{\partial^{2} W}{\partial x^{2}}\right)_{x=0, \ell}=0, \quad-b \leq y \leq b, \\
\left(\frac{\partial^{2} W}{\partial y^{2}}+\nu \frac{\partial^{2} W}{\partial x^{2}}\right)_{y= \pm b}=0, \quad 0 \leq x \leq \ell, \\
\left(\frac{\partial^{3} W}{\partial y^{3}}+(2-\nu) \frac{\partial^{3} W}{\partial x^{2} \partial y}\right)_{y= \pm b}=0, \quad 0 \leq x \leq \ell .
\end{gathered}
$$

Compare (3.16-3.18) and (3.20), on p. 46.

We multiply (4.17) by $W$ and perform integration over the domain $\Omega$ to obtain

$$
\begin{array}{r}
s^{2} \int_{\Omega} W^{2} \mathrm{~d} \Omega+2 s V_{0} \int_{\Omega} W \frac{\partial W}{\partial x} \mathrm{~d} \Omega+\left(V_{0}^{2}-C^{2}\right) \int_{\Omega} W \frac{\partial^{2} W}{\partial x^{2}} \mathrm{~d} \Omega \\
-\frac{T(y)}{m} \int_{\Omega} W \frac{\partial^{2} W}{\partial x^{2}} \mathrm{~d} \Omega+\frac{D}{m} \int_{\Omega} W \triangle^{2} W \mathrm{~d} \Omega=0 .
\end{array}
$$

Using the boundary conditions $(4.18-4.20)$ and performing integration by parts, we find the same result as in (3.11) and (3.12) on p. 45 : 


$$
\begin{aligned}
\int_{\Omega} W \frac{\partial W}{\partial x} \mathrm{~d} \Omega & =\int_{-b}^{b} \int_{0}^{\ell} W \frac{\partial W}{\partial x} \mathrm{~d} x \mathrm{~d} y \\
& =\int_{-b}^{b}\left[\frac{W^{2}(\ell, y)}{2}-\frac{W^{2}(0, y)}{2}\right] \mathrm{d} y \\
& =\quad 0, \\
\int_{\Omega} W \frac{\partial^{2} W}{\partial x^{2}} \mathrm{~d} \Omega & =-\int_{\Omega}\left(\frac{\partial W}{\partial x}\right)^{2} \mathrm{~d} \Omega .
\end{aligned}
$$

The non-homogeneous tension-related integral admits the following representation:

$$
\int_{\Omega} y W \frac{\partial^{2} W}{\partial x^{2}} \mathrm{~d} \Omega=-\int_{\Omega} y\left(\frac{\partial W}{\partial x}\right)^{2} \mathrm{~d} \Omega .
$$

We have

$$
\begin{aligned}
s^{2} \int_{\Omega} W^{2} \mathrm{~d} \Omega+ & \left(C^{2}-V_{0}^{2}\right) \int_{\Omega}\left(\frac{\partial W}{\partial x}\right)^{2} \mathrm{~d} \Omega+ \\
& +\frac{\alpha}{m} \int_{\Omega} y\left(\frac{\partial W}{\partial x}\right)^{2} \mathrm{~d} \Omega+\frac{D}{m} \int_{\Omega} W \triangle^{2} W \mathrm{~d} \Omega=0 .
\end{aligned}
$$

Two special cases, from which it is possible to draw further conclusions, will be considered. First, let $\alpha=0$ and $T_{x x}(x, y)=T_{0}$, i.e. one assumes homogeneous tension. In this case, as it was shown in Sect. 3.3.2, the following relation takes place:

$$
\int_{\Omega} W \triangle^{2} W \mathrm{~d} \Omega=\int_{\Omega}(\triangle W)^{2} \mathrm{~d} \Omega+2 \int_{0}^{\ell} Q_{y=b} \mathrm{~d} x .
$$

Above, the abbreviation

$$
Q=W \frac{\partial}{\partial y}(\triangle W)-\triangle W \frac{\partial W}{\partial y}
$$

has been used. Note that in (4.24) symmetry properties of the original partial differential equation were used to obtain this form of the $Q$ integral. Consequently, one has 


$$
\begin{aligned}
& \omega^{2}=-s^{2}= \\
& \quad \frac{\left(C^{2}-V_{0}^{2}\right) \int_{\Omega}\left(\frac{\partial W}{\partial x}\right)^{2} \mathrm{~d} \Omega+\frac{D}{m}\left[\int_{\Omega}(\triangle W)^{2} \mathrm{~d} \Omega+2 \int_{0}^{\ell} Q_{y=b} \mathrm{~d} x\right]}{\int_{\Omega} W^{2} \mathrm{~d} \Omega} .
\end{aligned}
$$

At the critical velocity, as can be seen from (4.26), the following relation between the critical velocity and the divergence mode holds:

$$
\left(V_{0}^{\mathrm{div}}\right)^{2}=C^{2}+\frac{D}{m} \frac{\int_{\Omega}(\Delta W)^{2} \mathrm{~d} \Omega+2 \int_{0}^{\ell} Q_{y=b} \mathrm{~d} x}{\int_{\Omega}\left(\frac{\partial W}{\partial x}\right)^{2} \mathrm{~d} \Omega} .
$$

In order to determine that $Q_{y=b}>0$ at this point, one needs to use the solution from the corresponding static problem, described in the next section for the general case. With that observation, we see that all integrals on the right side of (4.27) are positive, and it holds that

$$
\left(V_{0}^{\operatorname{div}}\right)^{2}>C^{2}
$$

It follows from (4.27) that if the bending rigidity of the web is negligibly small, then

$$
\left(V_{0 \mathrm{mem}}^{\mathrm{div}}\right)^{2}=C^{2}=\frac{T_{0}}{m} \text {. }
$$

In the one-dimensional case of axially travelling strings, this is a known result (see, e.g., Chang and Moretti 1991). From (4.29), we see that the same value of the critical velocity also applies to ideal membranes. The expression for $V_{0 \mathrm{mem}}^{\text {div }},(4.29)$, does not depend on $W$. Thus, for the special case of an ideal membrane under homogeneous tension, any combination of modes may occur at the critical velocity.

Consider now a second special case, where the bending rigidity of the axially moving plate is negligibly small, and the in-plane tension in the $x$ direction is positive. Thus we avoid compression and wrinkling considerations. Illustration can be seen in Fig. 4.1 on p. 84. We assume that

$$
D=0, \quad T_{0}>\alpha b,
$$

where the latter condition comes from the constraints

$$
T_{x x}(x, y)=T_{0}+\alpha y>0 \quad \text { and } \quad y \geq-b .
$$

In this case, the characteristic parameter $s$ is evaluated as

$$
\omega^{2}=-s^{2}=\frac{\left(C^{2}-V_{0}^{2}\right) \int_{\Omega}\left(\frac{\partial W}{\partial x}\right)^{2} \mathrm{~d} \Omega+\frac{\alpha}{m} \int_{\Omega} y\left(\frac{\partial W}{\partial x}\right)^{2} \mathrm{~d} \Omega}{\int_{\Omega} W^{2} \mathrm{~d} \Omega} .
$$


If a steady-state solution (divergence) exists, it will occur at velocity

$$
\left(V_{0}^{\text {div }}\right)^{2}=C^{2}+\frac{\alpha}{m} \frac{\int_{\Omega} y\left(\frac{\partial W}{\partial x}\right)^{2} \mathrm{~d} \Omega}{\int_{\Omega}\left(\frac{\partial W}{\partial x}\right)^{2} \mathrm{~d} \Omega} .
$$

Let us assume that the divergence mode $W$ is a real-valued function. Taking into account the expression in (4.33), and the fact that $y \geq-b$, we can estimate the divergence velocity (from below) as

$$
\left(V_{0}^{\text {div }}\right)^{2} \geq C^{2}-\frac{\alpha b}{m}=\frac{T_{0}-\alpha b}{m} .
$$

We see from (4.34) that as long as the condition for $T_{0}$ in (4.30) is fulfilled, we have $\left(V_{0}^{\text {div }}\right)^{2} \geq 0$, i.e., the value of $V_{0}^{\text {div }}$ is physically meaningful.

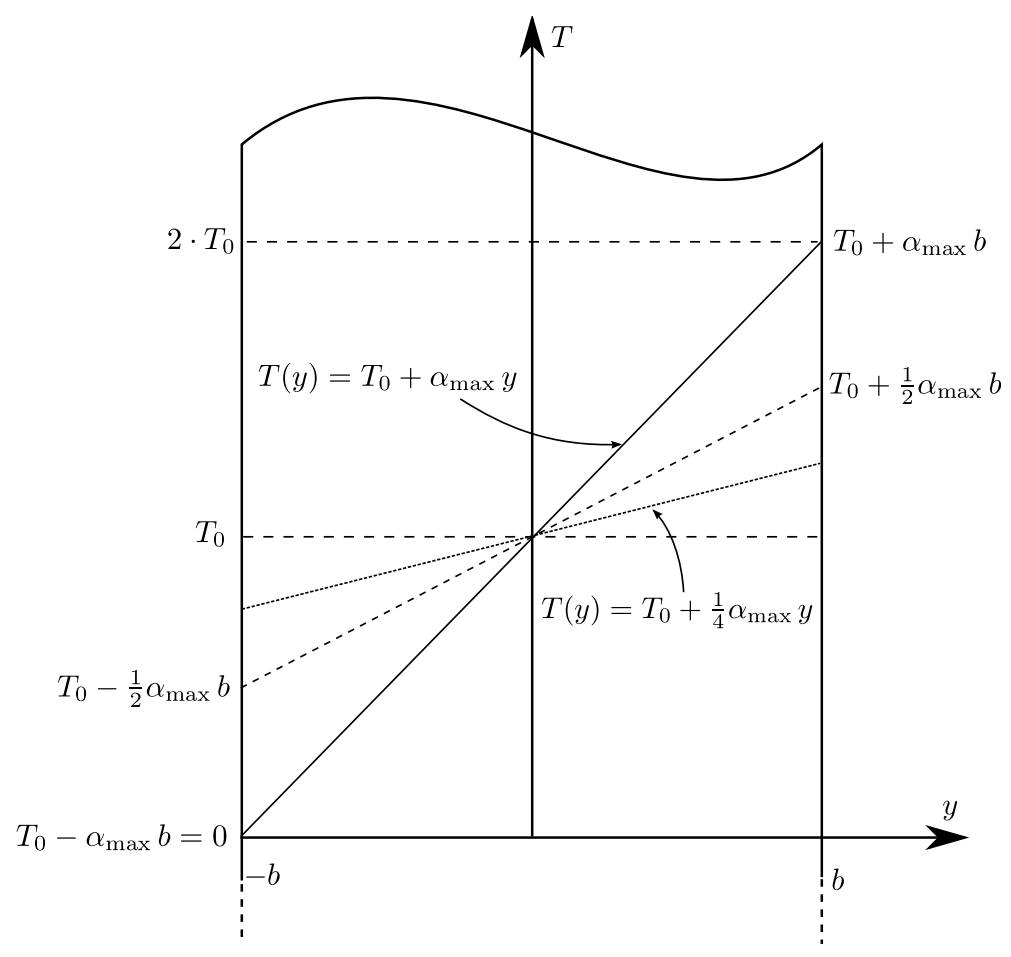

Fig. 4.2 The definition of $\alpha_{\max }$. It is the largest skew that retains $T(y) \geq 0$ across the whole domain, avoiding compression and wrinkling. 


\subsection{Solution of eigenvalue problem}

We will next consider the static instability of the travelling thin plate subjected to a linearly skewed tension profile. The treatment of the problem follows the same approach as in Sects. 3.4 and 3.5, where we analyzed the static instability of travelling isotropic and orthotropic plates under a under the assumption of a homogeneous tension profile.

\subsubsection{Transformation to ordinary differential equation}

The stationary eigenvalue problem of elastic instability consists of finding a nontrivial solution (mode) and the corresponding minimal eigenvalue of the following boundary-value problem. Consider the steady-state equation, corresponding to $s=0$ in the nonstationary problem in (4.17),

$$
\begin{aligned}
\left(V_{0}^{2}-C^{2}\right) \frac{\partial^{2} W}{\partial x^{2}}- & \frac{T(y)}{m} \frac{\partial^{2} W}{\partial x^{2}}+ \\
+ & \frac{D}{m}\left(\frac{\partial^{4} W}{\partial x^{4}}+2 \frac{\partial^{4} W}{\partial x^{2} \partial y^{2}}+\frac{\partial^{4} W}{\partial y^{4}}\right)=0, \quad(x, y) \in \Omega
\end{aligned}
$$

with the boundary conditions for $W$ in (4.18-4.20). From the latter condition in (4.30), we obtain a constraint for $\alpha$ :

$$
T(y)=\alpha y \quad \text { and } \quad \alpha<T_{0} / b .
$$

To determine the minimal eigenvalue $\lambda$ (see (3.39) on page 50) of the problem (4.18-4.20) and (4.35), and the corresponding eigenfunction, we apply the same representation as before:

$$
W=W(x, y)=f\left(\frac{y}{b}\right) \sin \left(\frac{\pi x}{\ell}\right),
$$

where $f(y / b)$ is an unknown function. It follows from (4.37) that the divergence form (steady-state solution) $W$ satisfies the boundary conditions (4.18).

As before, let us define the dimensionless quantities $\eta$ and $\mu$, given by (3.41) on page 50,

$$
\eta=\frac{y}{b}, \quad \mu=\frac{\ell}{\pi b},
$$

and the eigenvalue $\lambda$ as per (3.39),

$$
\lambda=\gamma^{2}=\frac{\ell^{2}}{\pi^{2} D}\left(m V_{0}^{2}-T_{0}\right) .
$$


By using the free-of-traction boundary conditions (4.19) and (4.20), the static equation (4.35) and the definition of $W,(4.37)$, we obtain the following eigenvalue problem for the unknown function $f(\eta)$ :

$$
\mu^{4} \frac{\mathrm{d}^{4} f}{\mathrm{~d} \eta^{4}}-2 \mu^{2} \frac{\mathrm{d}^{2} f}{\mathrm{~d} \eta^{2}}+(1-\lambda+\tilde{\alpha} \eta) f=0, \quad-1<\eta<1,
$$

where

$$
\tilde{\alpha}=\frac{b \ell^{2}}{\pi^{2} D} \alpha=\frac{b^{3} \mu^{2}}{D} \alpha .
$$

Equation (4.40) is considered with the boundary conditions

$$
\begin{gathered}
\mu^{2} \frac{\mathrm{d}^{2} f}{\mathrm{~d} \eta^{2}}-\nu f=0, \quad \eta= \pm 1 \quad \text { and } \\
\mu^{2} \frac{\mathrm{d}^{3} f}{\mathrm{~d} \eta^{3}}-(2-\nu) \frac{\mathrm{d} f}{\mathrm{~d} \eta}=0, \quad \eta= \pm 1
\end{gathered}
$$

which correspond to the free-of-traction boundary conditions of the original problem.

Equation (4.40) with the boundary conditions (4.42) and (4.43) constitutes a linear eigenvalue problem for $f$ with polynomial coefficients.

For an orthotropic material, it is possible to use problem (4.40), (4.42-4.43) in a straightforward way by setting the orthotropic in-plane shear modulus $G_{12}$ as the geometric average shear modulus

$$
G_{\mathrm{H}} \equiv \frac{\sqrt{E_{1} E_{2}}}{2\left(1+\sqrt{\nu_{12} \nu_{21}}\right)},
$$

and reducing the orthotropic problem into the isotropic one (see Timoshenko and Woinowsky-Krieger 1959).

Alternatively, if one wishes to keep $G_{12}$ as an independent material parameter, which is more accurate for some materials, it is possible to derive the corresponding eigenvalue problem for the orthotropic plate following the same procedure that was used above for the isotropic plate. Again, let the axial in-plane tension (4.1) take the form (4.12). As was noted, the value of $\alpha$ in (4.12) is constrained by (4.36). We have the following partial differential equation:

$$
\left(m V_{0}^{2}-T_{0}\right) \frac{\partial^{2} W}{\partial x^{2}}-\frac{T(y)}{m} \frac{\partial^{2} W}{\partial x^{2}}+D_{0} \mathcal{L}_{0}(w)=0,
$$

where the differential operator $\mathcal{L}_{0}(w)$ is given by $(3.4)$ on p. 43 ,

$$
\mathcal{L}_{0}(w)=\frac{D_{1}}{D_{0}} \frac{\partial^{4} w}{\partial x^{4}}+\frac{2 D_{3}}{D_{0}} \frac{\partial^{4} w}{\partial x^{2} \partial y^{2}}+\frac{D_{2}}{D_{0}} \frac{\partial^{4} w}{\partial y^{4}},
$$


and $D_{0}$ is an arbitrary normalization factor, which is convenient to take as $D_{0}=D_{1}$. The coefficients $D_{j}$ for $j=1,2,3$ are the orthotropic bending rigidities

$$
D_{1}=\frac{h^{3}}{12} C_{11}, \quad D_{2}=\frac{h^{3}}{12} C_{22}, \quad D_{3}=\frac{h^{3}}{12}\left(C_{12}+2 C_{66}\right),
$$

which were already given as (2.18), Sect. 2.1.3 (or see Timoshenko and Woinowsky-Krieger 1959, chap. 11). The $C_{i j}$ are the elastic moduli, (2.19).

The boundary conditions for $W$ are given in (4.18-4.20). However, in the free edge boundary conditions (4.19) and (4.20), instead of the isotropic free boundary coefficients $\nu$ and $2-\nu$, we must now use the orthotropic coefficients $\beta_{1}$ and $\beta_{2}$ (respectively) defined in (2.23) in the same way as in Sect. 2.1.3 (their definitions are repeated at the end of this subsection for convenience).

In Sect. 2.2, the in-plane tension field for an orthotropic plate in the case of a linear tension distribution was solved with the help of the Airy stress function. See equations (2.50) and (2.51).

To determine the minimal eigenvalue $\lambda$ (4.39), and the corresponding eigenfunction, of the problem (4.44) with boundary conditions (4.18-4.20) (modified for the orthotropic case as explained), we apply the representation (4.37). By using the dimensionless quantities $\eta$ and $\mu$ in (4.38), the freeof-traction boundary conditions (4.19) and (4.20), with the relations (4.39), (4.44) and (4.37), we obtain the eigenvalue problem for the orthotropic case:

$$
\begin{gathered}
\mu^{4} H_{2} \frac{\mathrm{d}^{4} f}{\mathrm{~d} \eta^{4}}-2 \mu^{2} H_{3} \frac{\mathrm{d}^{2} f}{\mathrm{~d} \eta^{2}}+\left(H_{1}-\lambda-\bar{\alpha} \eta\right) f=0, \quad-1<\eta<1, \\
\mu^{2} \frac{\mathrm{d}^{2} f}{\mathrm{~d} \eta^{2}}-\beta_{1} f=0, \quad \eta= \pm 1 \quad \text { and } \\
\mu^{2} \frac{\mathrm{d}^{3} f}{\mathrm{~d} \eta^{3}}-\beta_{2} \frac{\mathrm{d} f}{\mathrm{~d} \eta}=0, \quad \eta= \pm 1 .
\end{gathered}
$$

In (4.45), the dimensionless tension profile skew parameter is defined as

$$
\bar{\alpha}=\frac{b \ell^{2}}{\pi^{2} D_{0}} \alpha=\frac{b^{3} \mu^{2}}{D_{0}} \alpha
$$

and the $H_{j}$ are the dimensionless bending rigidities defined by (3.91), Sect. 3.5 , repeated here for convenience:

$$
H_{1}=\frac{D_{1}}{D_{0}}, \quad H_{2}=\frac{D_{2}}{D_{0}}, \quad H_{3}=\frac{D_{3}}{D_{0}} .
$$

As before, $D_{0}$ is the normalization factor for the bending rigidities and can be chosen arbitrarily. A convenient choice is $D_{0}=D_{1}$. In (4.46) and (4.47), the $\beta_{j}$ are defined in (2.23) in Sect. 2.1.3, also repeated here for convenience: 


$$
\begin{gathered}
\beta_{1}=\nu_{12}, \\
\beta_{2}=\nu_{12}+\frac{4 G_{12}}{E_{2}}\left(1-\nu_{12} \nu_{21}\right) .
\end{gathered}
$$

Again, we have a linear eigenvalue problem with polynomial coefficients.

For the rest of this chapter, we will concentrate on the isotropic case.

\subsubsection{Numerical analysis}

We will proceed with a numerical solution of the eigenvalue problem for the isotropic elastic plate. Finite differences will be used, with virtual points added to the ends of the domain to enforce the boundary conditions.

As the considered problem is linear in $f$, the discretization will lead to a standard discrete linear eigenvalue problem representing (4.40):

$$
\mathbf{A f}=\lambda \mathbf{f} .
$$

Equation (4.49) does not yet include the boundary conditions (4.42)-(4.43). Because the boundary conditions are homogeneous, it is possible to add them to the discrete system by rewriting the original discrete problem (4.49) as a generalized linear eigenvalue problem,

$$
\mathbf{A f}=\lambda \mathbf{B f},
$$

where $\mathbf{B}$ is an identity matrix with the first two and last two rows zeroed out. In (4.50), the first two and the last two rows of $A$ contain discrete representations of the boundary conditions (4.42)-(4.43).

The details are as follows. Equations (4.40)-(4.43) are to be discretized. The standard central difference formulas, of second-order asymptotic accuracy, for the first four derivatives on a uniform grid are

$$
\begin{aligned}
\frac{\partial f_{j}}{\partial \eta} & \approx \frac{f_{j+1}-f_{j-1}}{2(\Delta \eta)}, \\
\frac{\partial^{2} f_{j}}{\partial \eta^{2}} & \approx \frac{f_{j+1}-2 f_{j}+f_{j-1}}{(\Delta \eta)^{2}}, \\
\frac{\partial^{3} f_{j}}{\partial \eta^{3}} & \approx \frac{f_{j+2}-2 f_{j+1}+2 f_{j-1}-f_{j-2}}{2(\Delta \eta)^{3}}, \\
\frac{\partial^{4} f_{j}}{\partial \eta^{4}} & \approx \frac{f_{j+2}-4 f_{j+1}+6 f_{j}-4 f_{j-1}+f_{j-2}}{(\Delta \eta)^{4}},
\end{aligned}
$$

where $f \equiv f(x)$ is the function to be differentiated, $f_{j} \equiv f\left(\eta_{j}\right)$, and $\Delta \eta$ is the grid spacing. 
When the derivatives in (4.40) are replaced by the discrete approximations (4.52) and (4.54) for each grid point $\eta_{j}$, we obtain the discrete equation system for the interior of the domain. The $\bar{\alpha} \eta$ term is handled by substituting in the coordinate of the $j$ th grid point, $\eta_{j}=j(\Delta \eta)$. Then the discrete equations are collected into matrix form, and the $\lambda \mathbf{f}$ term is moved to the right-hand side.

The boundary conditions (4.42)-(4.43) are then handled by adding two virtual points at each end of the domain. Applying (4.51)-(4.54) to the boundary conditions produces discrete equations connecting the function values at the virtual points to those inside the domain.

If we number the points starting at 1 at the first (outermost) virtual point at the left end of the domain, the final left-hand side matrix becomes

$$
\mathbf{A} \equiv \mathbf{A}_{4}+\mathbf{A}_{2}+\mathbf{A}_{0}+\mathbf{L}_{1}+\mathbf{L}_{2}+\mathbf{L}_{3}+\mathbf{L}_{4},
$$

where the terms $\mathbf{A}_{m}$ correspond to equation (4.40), and are given by

$$
\begin{aligned}
& \mathbf{A}_{4} \equiv \frac{\mu^{4}}{(\Delta \eta)^{4}}\left[\begin{array}{ccccccc}
0 & \ldots & & & & \ldots & 0 \\
0 & \ldots & & & & \ldots & 0 \\
1 & -4 & 6 & -4 & 1 & & \\
& \ddots & \ddots & \ddots & \ddots & \ddots & \\
& & 1 & -4 & 6 & -4 & 1 \\
0 & \ldots & & & & \ldots & 0 \\
0 & \ldots & & & & \ldots & 0
\end{array}\right] \\
& \mathbf{A}_{2} \equiv-\frac{2 \mu^{2}}{(\Delta \eta)^{2}}\left[\begin{array}{rrrrrrrr}
0 & \ldots & & & & \ldots & 0 \\
0 & \ldots & & & & \ldots & 0 \\
0 & 1 & -2 & 1 & & & \\
& & \ddots & \ddots & \ddots & & \\
& & & 1 & -2 & 1 & 0 \\
0 & \ldots & & & & \ldots & 0 \\
0 & \ldots & & & & \ldots & 0
\end{array}\right] \\
& \mathbf{A}_{0} \equiv\left[\begin{array}{cccccccc}
0 & \ldots & & & \ldots & 0 \\
0 & 0 & \ldots & & & \ldots & 0 \\
0 & 0 & a_{k} & & & \ldots & 0 \\
& & & \ddots & & & \\
& & & & & a_{k} & 0 & 0 \\
0 & \ldots & & & \ldots & 0 & 0 \\
0 & \ldots & & & & \ldots & 0
\end{array}\right]
\end{aligned}
$$

where

$$
a_{k} \equiv 1+\bar{\alpha}[-1+(k-3)(\Delta \eta)]
$$


and $k$ denotes the row number of the matrix $\mathbf{A}_{0}$. The first contribution in $\mathbf{A}_{0}$ is $1-\bar{\alpha}$ (on row 3 , corresponding to the point at $\eta=-1$ ), and the last is $1+\bar{\alpha}$ (third last row, corresponding to $\eta=+1$ ).

Empty entries in the matrices denote zeroes; some zeroes are displayed explicitly to show more clearly where the nonzero entries belong.

The terms $\mathbf{L}_{m}$ correspond to the boundary conditions (4.42)-(4.43), and are given by

$$
\begin{aligned}
& \mathbf{L}_{1} \equiv\left[\begin{array}{ccccc}
0 & \ldots & & & \ldots 0 \\
0 & \mu^{2} /(\Delta \eta)^{2} & -2 \mu^{2} /(\Delta \eta)^{2}-\nu & \mu^{2} /(\Delta \eta)^{2} & \ldots 0 \\
0 & \ldots & \ldots & \ldots & \ldots 0 \\
0 & \ldots & \ldots & \ldots & \ldots 0 \\
0 & \ldots & \ldots & \ldots & \ldots 0 \\
0 & \ldots & & & \ldots 0 \\
0 & \ldots & & & \ldots 0
\end{array}\right], \\
& \mathbf{L}_{2} \equiv\left[\begin{array}{cccccc}
-\mu^{2} /\left[2(\Delta \eta)^{3}\right] & \chi & 0 & -\chi & \mu^{2} /\left[2(\Delta \eta)^{3}\right] & 0 \ldots 0 \\
0 & \ldots & & \ldots 0 \\
0 & \ldots & & \ldots 0 \\
0 & \ldots & & \ldots 0 \\
0 & \ldots & & \ldots 0 \\
0 & \ldots & & \ldots 0 \\
0 & \ldots & & \ldots
\end{array}\right] \text {, }
\end{aligned}
$$

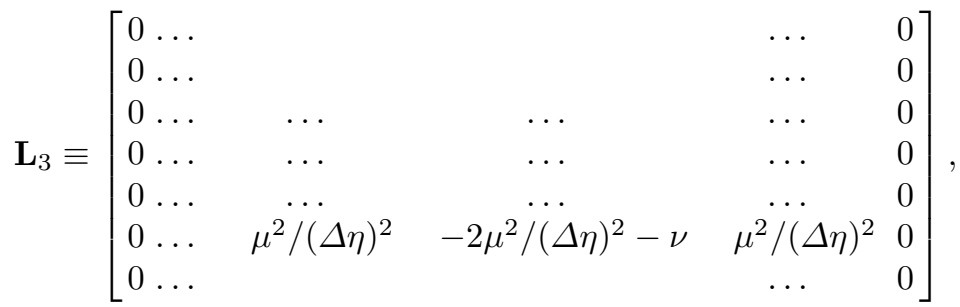

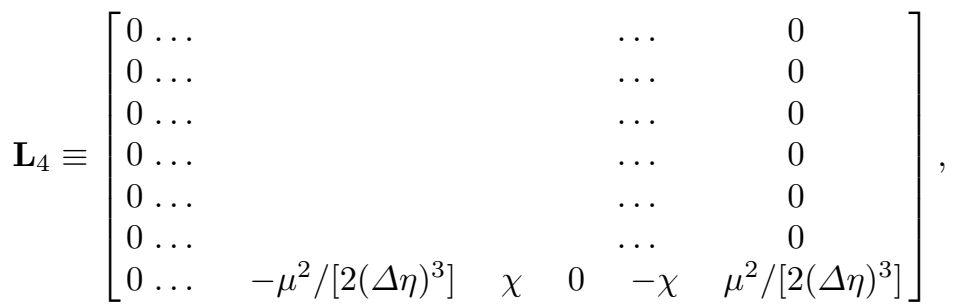

where in $\mathbf{L}_{2}$ and $\mathbf{L}_{4}$ we use the notation

$$
\chi \equiv \mu^{2} /(\Delta \eta)^{3}+(2-\nu) /[2(\Delta \eta)] .
$$

The matrices $\mathbf{L}_{1}$ and $\mathbf{L}_{3}$ correspond to the boundary condition (4.42) at the left and right endpoints of the domain, respectively, while $\mathbf{L}_{2}$ and $\mathbf{L}_{4}$ correspond to (4.43). 
Finally, the discrete problem (4.50) is completed by defining

$$
\mathbf{B} \equiv\left[\begin{array}{cccccccc}
0 & \ldots & & & & \ldots & 0 \\
0 & 0 & \ldots & & & \ldots & 0 \\
0 & 0 & 1 & & & \ldots & 0 \\
& & & \ddots & & & \\
0 & \ldots & & & 1 & 0 & 0 \\
0 & \ldots & & & \ldots & 0 & 0 \\
0 & \ldots & & & & \ldots & 0
\end{array}\right]
$$

which enforces the homogeneous boundary conditions (4.42)-(4.43), represented by the first two and last two rows of the discrete equation system.

In order to solve the original problem, we compute the solution of (4.50), discard eigenvalues of infinite magnitude, which result from our way of handling the boundary conditions, and then extract the smallest eigenvalue $\lambda$ and its corresponding eigenvector $\mathbf{f}$. The first two and last two components of the eigenvector are discarded, because they represent the function values at virtual points that were generated from the boundary conditions. Finally, the buckling mode (divergence mode) $W(x, y)$ is constructed using the equation

$$
W(x, y)=f\left(\frac{\pi y}{\ell}\right) \sin \left(\frac{\pi x}{\ell}\right) .
$$

Below, numerical results are shown for some practically interesting choices of problem parameters. The physical parameters used in the examples are presented in Table 4.1. These parameter values approximately correspond to some paper materials, within the limitations of the isotropic model.

Table 4.1 Physical parameters used in the numerical examples.

\begin{tabular}{llllll}
\hline$T_{0}($ tension at $y=0)$ & $m$ & $\ell$ & $2 b$ & $h$ & $E$ \\
\hline $500 \mathrm{~N} / \mathrm{m}$ & $0.08 \mathrm{~kg} / \mathrm{m}^{2}$ & $0.1 \mathrm{~m}$ & $1 \mathrm{~m}$ & $10^{-4} \mathrm{~m}$ & $10^{9} \mathrm{~N} / \mathrm{m}^{2}$ \\
\hline
\end{tabular}

Table 4.2 Critical divergence velocities $V_{0}^{\text {div }}$ for example cases. Note that $\tilde{\alpha}_{\text {max }}$ is different for each value of $\nu$. (Banichuk et al 2013)

\begin{tabular}{lllll}
\hline & $\tilde{\alpha}$ & & & \\
\cline { 2 - 5 }$\nu$ & 0 & $10^{-6} \tilde{\alpha}_{\max }$ & $10^{-4} \tilde{\alpha}_{\max }$ & $10^{-2} \tilde{\alpha}_{\max }$ \\
\hline 0 & 79.0634 & 79.0634 & 79.0605 & 78.6892 \\
0.1 & 79.0635 & 79.0635 & 79.0605 & 78.6886 \\
0.3 & 79.0640 & 79.0640 & 79.0609 & 78.6876 \\
0.5 & 79.0652 & 79.0652 & 79.0618 & 78.6870 \\
\hline
\end{tabular}


Various values of the Poisson ratio $\nu$ and the tension profile skew parameter $\tilde{\alpha}$ are used in the examples. For the Poisson ratio, the values $0,0.1,0.3$ and 0.5 are used. The values of $\alpha / \alpha_{\max }$ (or $\tilde{\alpha} / \tilde{\alpha}_{\max }$ ) are $0,10^{-6}, 10^{-4}$ and $10^{-2}$, where $\alpha_{\max }$ corresponds to the upper limit imposed by the constraint (4.36), $\alpha<T_{0} / b$. Note that $\tilde{\alpha}_{\max }$ depends on $\nu$, via $D$. In Table 4.2 , critical divergence velocities are presented for these cases. The analytical solution for $\tilde{\alpha}=0$ for the same geometric and material parameters (see (3.39), (3.54-3.56) on pages 50 and 52) matches the values in the first column of the table.
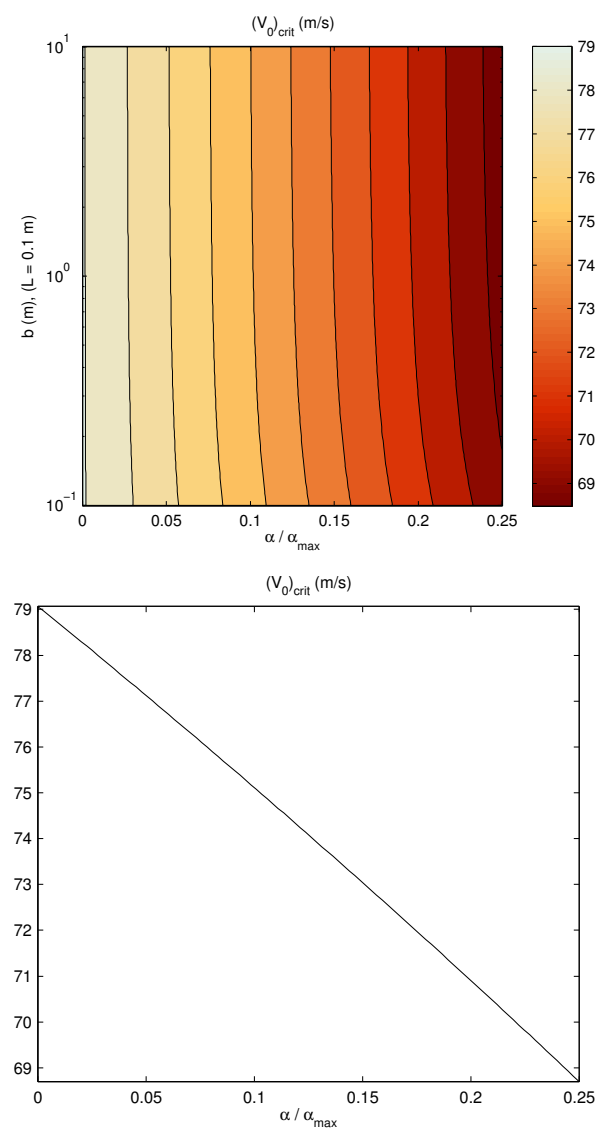

Fig. 4.3 Top: Critical plate velocity $\left(V_{0}\right)_{\text {crit }}$ with respect to the tension profile skew parameter $\alpha$ and plate half-width $b$. Note the logarithmic scale for $b$. The plate length is constant $(\ell=0.1 \mathrm{~m})$. Bottom: The critical velocity plotted with respect to the tension profile skew parameter $(\ell=0.1 \mathrm{~m}, 2 b=1 \mathrm{~m})$. (Reproduced from Banichuk et al 2013)

The results for the transverse displacement are shown in Figs. $4.4-4.6$. In each figure, $\nu$ is fixed. Fig. 4.4 is divided into two parts. Both parts of the figure are further divided into four subfigures. Each of these four subfigures 
shows the results for a different value of the skew parameter $\tilde{\alpha}$. In the upper four subfigures, $f(\eta)$ is plotted, showing a slice of the out-of-plane displacement from one free edge to the other at $x=\ell / 2$. Tension increases toward positive $\eta$. The total out-of-plane displacement in the whole domain $\Omega$, from equation $W=f(\pi y / \ell) \sin (\pi x / \ell)$, is shown in the lower four subfigures. Note the orientation of the axes. In Figs. 4.5 and 4.6, the four subfigures show the slices of the out-of-plane displacement at $x=\ell / 2$ for the limit cases $\nu=0$ and $\nu=0.5$, in analogous order.

From Figs. $4.3-4.6$ and Table 4.2, three conclusions are apparent. First, it is seen that inhomogeneities in the tension profile may significantly decrease the critical velocities. Up to a $20 \%$ tension inhomogeneity between the midpoint and edges causes a decrease in critical velocity of $10 \%$. It is also seen that a wider plate is more sensitive to tension inhomogeneities. Secondly, by comparing Figs. $4.4-4.6$, it is observed that materials with a larger Poisson ratio tend to exhibit a higher degree of sensitivity to inhomogeneities in the tension profile.

Finally, we see that even for the smallest inhomogeneity in the examples (one part in $10^{6}$ ), for the problem parameters considered the buckling mode (divergence mode) changes completely. Thus, from a practical point of view, although studies of the homogeneous tension case can predict the critical velocity relatively accurately, the obtained results indicate that if one wishes to predict the divergence shape, even a small inhomogeneity in the tension must be accounted for.

The sensitivity to the inhomogeneity is affected also by the tension at midpoint $T_{0}$. The higher the tension, the more sensitive the system is to small inhomogeneities. This effect is shown in Fig. 4.7. The subfigure on the bottom left of this figure corresponds to the subfigure at the top right of Fig. 4.4. We see that with $\nu=0.3, \tilde{\alpha}=10^{-6} \tilde{\alpha}_{\max }$, and the values of the other parameters fixed to those given at the beginning of this section, the sensitivity is very high already at $T_{0}=500 \mathrm{~N} / \mathrm{m}$, which is realistic in the application of paper production.

It should be noted that as far as geometric parameters are concerned, the divergence shape is a function of not only the aspect ratio $\ell / 2 b$, but also the overall scale. Even for the same aspect ratio, scaling $\ell$ (and also $b$ to keep the same aspect ratio) changes the divergence shape. This effect occurs even if $h$ is scaled by the same amount as $\ell$ and $b$. Thus, it should be emphasized that the results in Figs. $4.4-4.7$ only represent the specific case of plates with the dimensions $\ell \times 2 b \times h=0.1 \mathrm{~m} \times 1 \mathrm{~m} \times 10^{-4} \mathrm{~m}$. 

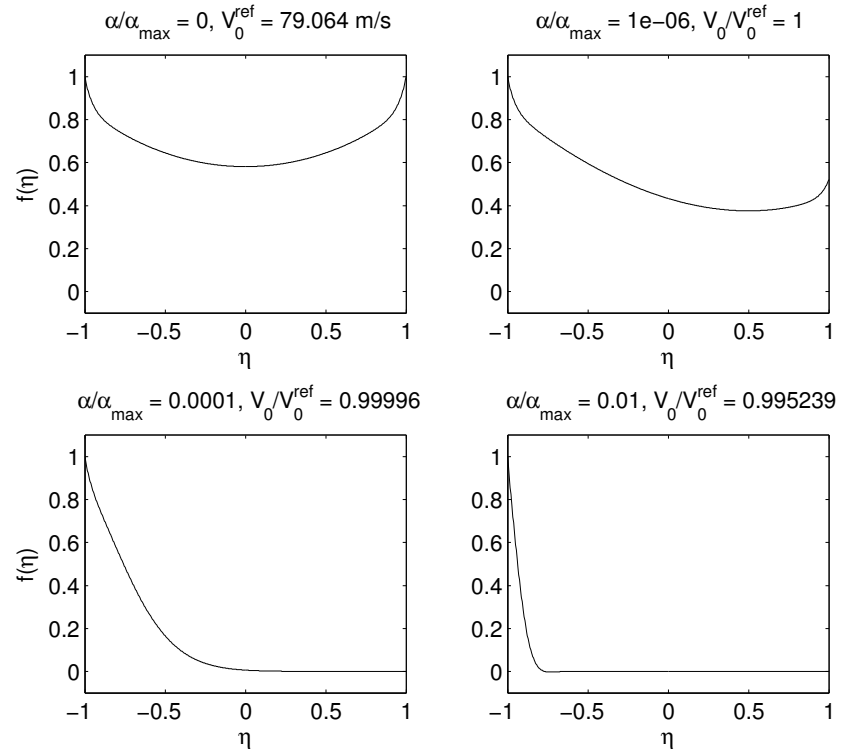

$\alpha / \alpha_{\max }=0.01, V_{0} / V_{0}^{\text {ref }}=0.995239$
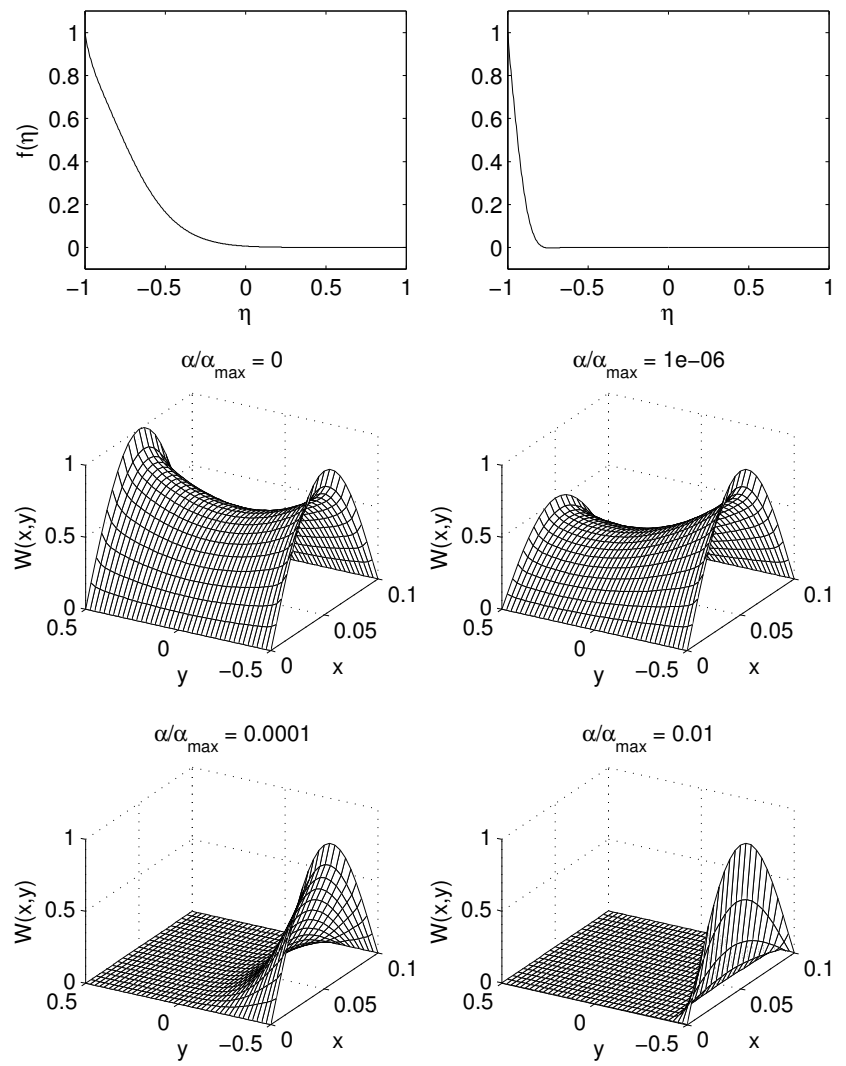

Fig. 4.4 Out-of-plane displacement of an axially travelling pinned-free plate with dimensions $\ell=0.1 \mathrm{~m}$ (length), $2 b=1 \mathrm{~m}$ (width), $h=10^{-4} \mathrm{~m}$ (thickness). Poisson ratio $\nu=0.3$. Tension profile skew parameter $\alpha / \alpha_{\max }=0,10^{-6}, 10^{-4}, 10^{-2}$. (Reproduced from Banichuk et al 2013) 

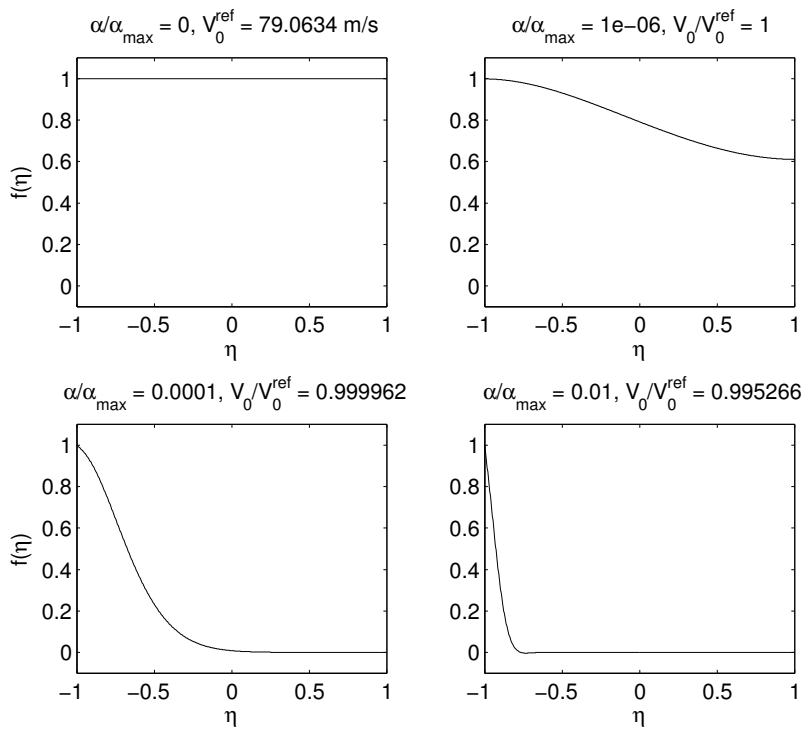

Fig. 4.5 Out-of-plane displacement of an axially travelling pinned-free plate at $x=\ell / 2$ with dimensions $\ell=0.1 \mathrm{~m}$ (length), $2 b=1 \mathrm{~m}$ (width), $h=10^{-4} \mathrm{~m}$ (thickness). Poisson ratio $\nu=0$. Tension profile skew parameter $\alpha / \alpha_{\max }=0,10^{-6}, 10^{-4}, 10^{-2}$. (Reproduced from Banichuk et al 2013)
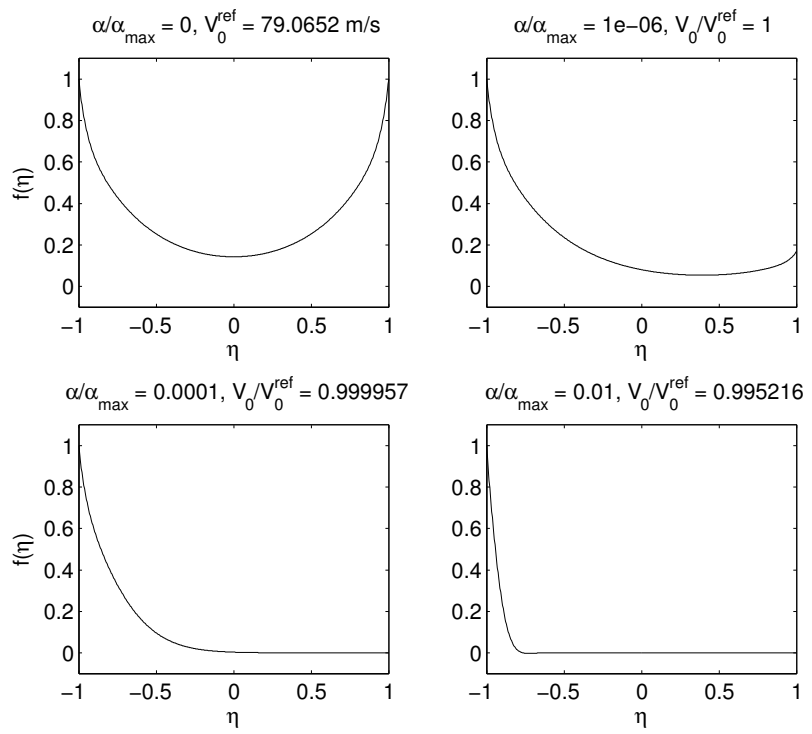

Fig. 4.6 Out-of-plane displacement of an axially travelling pinned-free plate at $x=\ell / 2$ with dimensions $\ell=0.1 \mathrm{~m}$ (length), $2 b=1 \mathrm{~m}$ (width), $h=10^{-4} \mathrm{~m}$ (thickness). Poisson ratio $\nu=0.5$. Tension profile skew parameter $\alpha / \alpha_{\max }=0,10^{-6}, 10^{-4}, 10^{-2}$. (Reproduced from Banichuk et al 2013) 

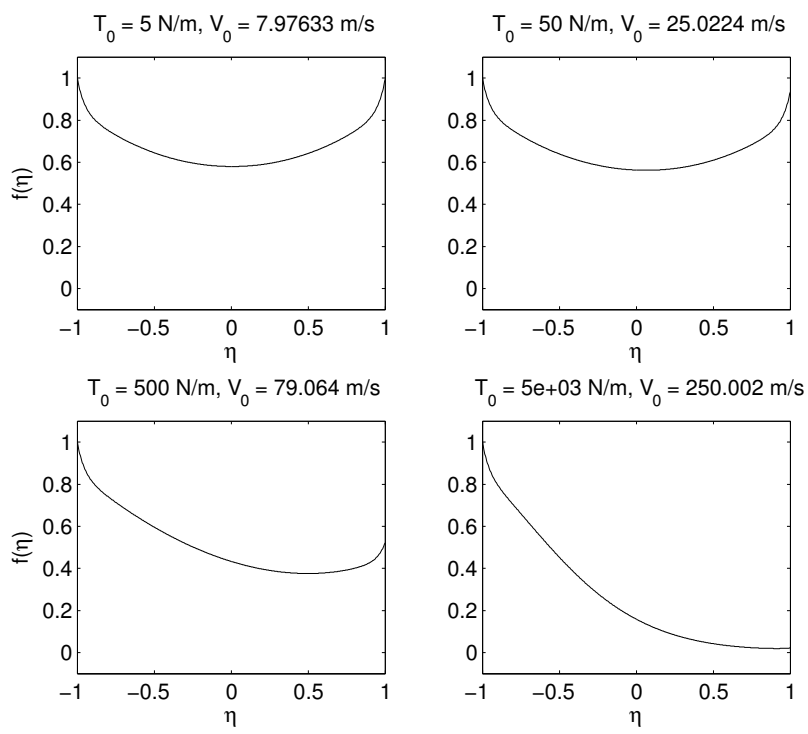

Fig. 4.7 Out-of-plane displacement of an axially travelling pinned-free plate at $x=\ell / 2$ with dimensions $\ell=0.1 \mathrm{~m}$ (length), $2 b=1 \mathrm{~m}$ (width), $h=10^{-4} \mathrm{~m}$ (thickness). Poisson ratio $\nu=0.3$, tension profile skew parameter $\alpha / \alpha_{\max }=10^{-6}$. Midpoint tension $T_{0}=$ $5,50,500$ and $5000 \mathrm{~N} / \mathrm{m}$. (Reproduced from Banichuk et al 2013)

\section{References}

Banichuk N, Jeronen J, Neittaanmäki P, Saksa T, Tuovinen T (2013) Theoretical study on travelling web dynamics and instability under non-homogeneous tension. International Journal of Mechanical Sciences 66C:132-140, URL http://dx.doi.org/10.1016/j.ijmecsci.2012.10.014

Bolotin VV (1963) Nonconservative Problems of the Theory of Elastic Stability. Pergamon Press, New York

Chang YB, Moretti PM (1991) Interaction of fluttering webs with surrounding air. TAPPI Journal 74(3):231-236

Timoshenko SP, Woinowsky-Krieger S (1959) Theory of plates and shells, 2nd edn. New York : Tokyo : McGraw-Hill, ISBN 0-07-085820-9 


\title{
Chapter 5 Travelling panels made of viscoelastic material
}

\begin{abstract}
In this chapter, our focus is to analyse the behaviour of moving panels using viscoelastic materials. As the reader will have noticed, all the models discussed in previous chapters have concerned the case of a purely elastic material. The deformation of an elastic material depends only on the applied forces; it has no explicit time dependence. Paper, however, is a more complicated material: it is viscoelastic. In addition to elastic properties, it has also time-dependent viscous properties, which cause the phenomena of creep and relaxation (see, e.g., Alava and Niskanen 2006). One of the simplest models for a viscoelastic solid is the Kelvin-Voigt model, which consists of a linear spring and a dashpot (damper) connected in parallel. In this chapter, we will use dynamic (modal) analysis to investigate the stability of an axially moving viscoelastic Kelvin-Voigt panel. The material derivative will be used in the viscoelastic constitutive relations. A similar analysis has been performed in the papers by Marynowski and Kapitaniak (2002) and Lee and Oh (2005), but using the partial time derivative in the viscoelastic constitutive relations. We will present comparisons of the results using the material derivative with the results obtained using the partial time derivative.
\end{abstract}

\subsection{Modelling of moving viscoelastic panels}

Consider an axially moving thin plate, made of viscoelastic material, in a Cartesian coordinate system. The plate is assumed to undergo cylindrical transverse deformation, that is, the transverse displacement does not vary in the $y$ direction. In such a case, the panel model can be used (Timoshenko and Woinowsky-Krieger 1959; Bisplinghoff and Ashley 1962). See Fig. 5.1.

The panel is supported at $x=0$ and $x=\ell$, and the length of the unsupported interval is $\ell$. The axial velocity of the panel is assumed to be constant 

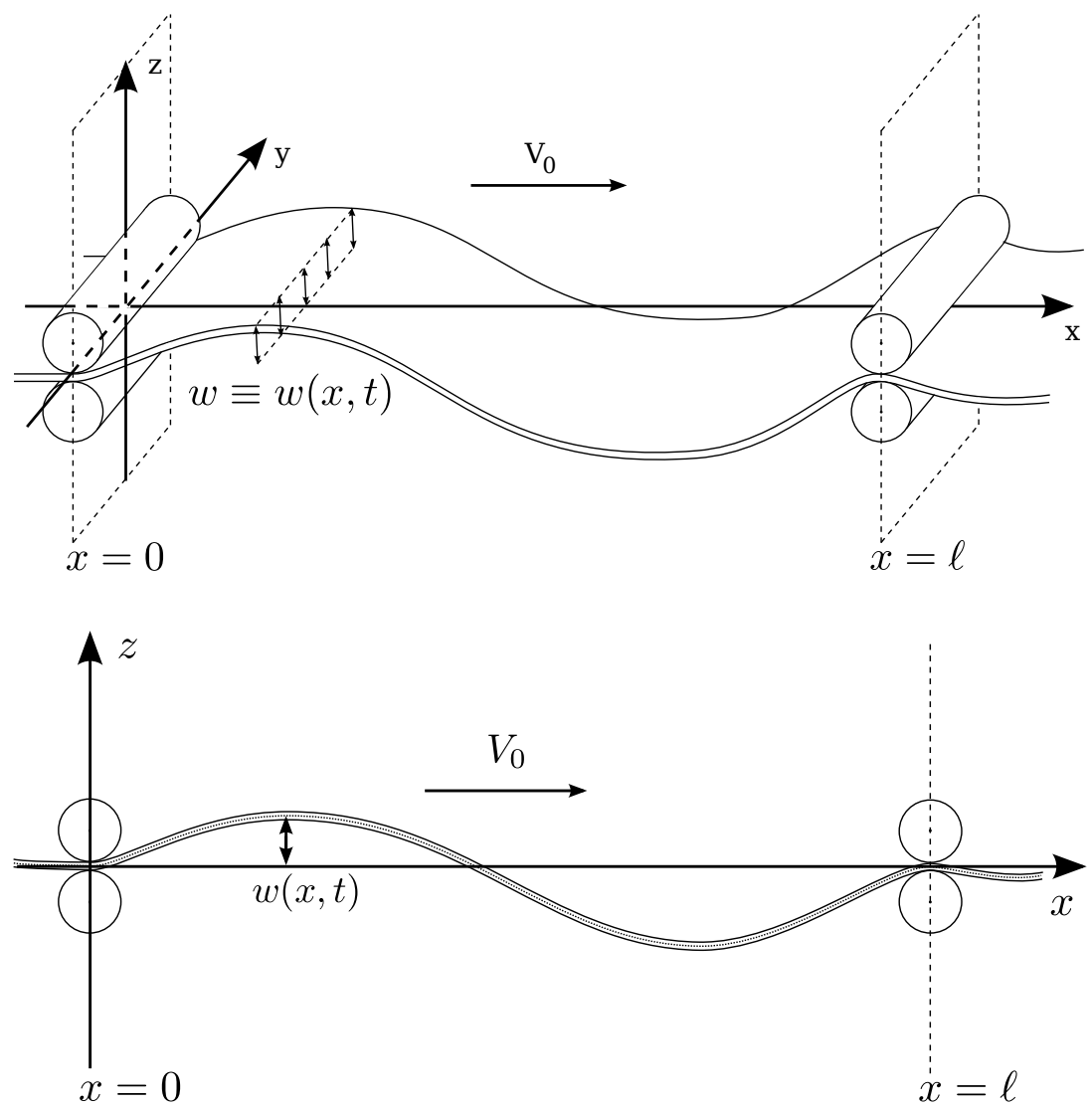

Fig. 5.1 Top: A plate travelling between two fixed supports, undergoing cylindrical deformation. Bottom: The corresponding travelling panel. (Reproduced from Saksa et al (2012) with permission from Elsevier)

and denoted by $V_{0}$. The transverse displacement is described by the function $w=w(x, t)$.

The viscoelasticity of the material is described using the rheological Kelvin-Voigt model, which consists of an elastic spring and a viscous damper connected in parallel. The spring element is described by the elastic parameters $E$ and $\nu$, and the damper by the viscous damping coefficient $\eta$ and the Poisson ratio for viscosity $\mu$. See Fig. 5.2.

Since, we consider a panel, i.e. a two-dimensional plate with the assumption that its transverse displacement does not vary in the $y$ direction, we will first present the stress-strain relations of bending for the plate and then reduce them to the one-dimensional relations. Under assumption of plane stress, the constitutive stress-strain relations for elastic material are 


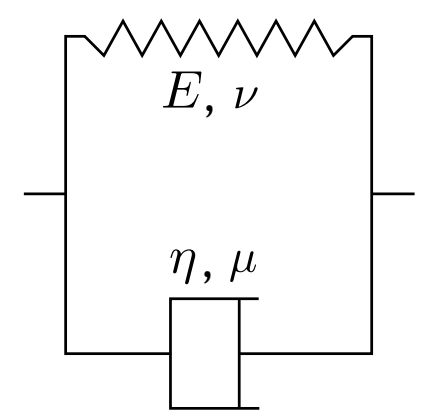

Fig. 5.2 The Kelvin-Voigt viscoelastic material model. Combining various numbers of springs and dashpots it is possible to generate more advanced and complex models for many situations.

$$
\begin{aligned}
\sigma_{x} & =\frac{E}{1-\nu^{2}}\left(\varepsilon_{x}+\nu \varepsilon_{y}\right), \\
\sigma_{y} & =\frac{E}{1-\nu^{2}}\left(\nu \varepsilon_{x}+\varepsilon_{y}\right), \\
\tau_{x y} & =\frac{E}{2(1+\nu)} \gamma_{x y},
\end{aligned}
$$

where $\sigma_{x}, \sigma_{y}$ are the normal stresses, and $\tau_{x y}$ the shear stress due to bending. The bending strains $\varepsilon_{x}$ and $\varepsilon_{y}$ and the shear strain $\gamma_{x y}$ for small deformations are defined as (Timoshenko and Woinowsky-Krieger 1959)

$$
\begin{aligned}
\varepsilon_{x} & =-z \frac{\partial^{2} w}{\partial x^{2}}, \\
\varepsilon_{y} & =-z \frac{\partial^{2} w}{\partial y^{2}}, \\
\gamma_{x y} & =-2 z \frac{\partial^{2} w}{\partial x \partial y} .
\end{aligned}
$$

The relations in (5.1) are also known as the generalized Hooke's law; compare with the generalized Hooke's law for the in-plane strains of orthotropic material, (2.32-2.34), in Sect. 2.2.

Similar constitutive relations as $(5.1)$ for a viscous Newtonian fluid can be written, corresponding to the viscous damper in the Kelvin-Voigt model (see also, e.g., Sobotka 1984): 


$$
\begin{aligned}
\sigma_{x} & =\frac{\eta}{1-\mu^{2}}\left(\frac{\mathrm{d} \varepsilon_{x}}{\mathrm{~d} t}+\mu \frac{\mathrm{d} \varepsilon_{y}}{\mathrm{~d} t}\right), \\
\sigma_{y} & =\frac{\eta}{1-\mu^{2}}\left(\mu \frac{\mathrm{d} \varepsilon_{x}}{\mathrm{~d} t}+\frac{\mathrm{d} \varepsilon_{y}}{\mathrm{~d} t}\right), \\
\tau_{x y} & =\frac{\eta}{2(1+\mu)} \frac{\mathrm{d} \gamma_{x y}}{\mathrm{~d} t} .
\end{aligned}
$$

In (5.3), the derivatives $\mathrm{d} / \mathrm{d} t$ denote material derivatives,

$$
\frac{\mathrm{d}(\cdot)}{\mathrm{d} t}=\frac{\partial(\cdot)}{\partial t}+V_{0} \frac{\partial(\cdot)}{\partial x} .
$$

The stress-strain relations for the axially moving Kelvin-Voigt plate under assumption of plane stress are obtained by superposing the stress-strain relations in (5.1) and (5.3) and by expanding the material derivatives:

$$
\begin{aligned}
\sigma_{x} & =\frac{E}{1-\nu^{2}}\left(\varepsilon_{x}+\nu \varepsilon_{y}\right) \\
& +\frac{\eta}{1-\mu^{2}}\left[\left(\frac{\partial \varepsilon_{x}}{\partial t}+V_{0} \frac{\partial \varepsilon_{x}}{\partial x}\right)+\mu\left(\frac{\partial \varepsilon_{y}}{\partial t}+V_{0} \frac{\partial \varepsilon_{y}}{\partial x}\right)\right] \\
\sigma_{y} & =\frac{E}{1-\nu^{2}}\left(\nu \varepsilon_{x}+\varepsilon_{y}\right) \\
& +\frac{\eta}{1-\mu^{2}}\left[\mu\left(\frac{\partial \varepsilon_{x}}{\partial t}+V_{0} \frac{\partial \varepsilon_{x}}{\partial x}\right)+\left(\frac{\partial \varepsilon_{y}}{\partial t}+V_{0} \frac{\partial \varepsilon_{y}}{\partial x}\right)\right] \\
\tau_{x y} & =\frac{E}{2(1+\nu)} \gamma_{x y}+\frac{\eta}{2(1+\mu)}\left(\frac{\partial \gamma_{x y}}{\partial t}+V_{0} \frac{\partial \gamma_{x y}}{\partial x}\right) .
\end{aligned}
$$

Denoting $\sigma=\sigma_{x}$ and $\varepsilon=\varepsilon_{x}$, and assuming small cylindrical deformations (hence $\varepsilon_{y}=-z \partial^{2} w / \partial y^{2}=0$ ), the relations (5.4) are reduced to

$$
\sigma=\frac{E}{1-\nu^{2}} \varepsilon+\frac{\eta}{1-\mu^{2}}\left(\frac{\partial \varepsilon}{\partial t}+V_{0} \frac{\partial \varepsilon}{\partial x}\right)
$$

For the bending moment $M=M_{x}$, we have

$$
M=\int_{-h / 2}^{h / 2} z \sigma \mathrm{d} z=-\left[D \frac{\partial^{2} w}{\partial x^{2}}+\Upsilon\left(\frac{\partial^{3} w}{\partial x^{2} \partial t}+V_{0} \frac{\partial^{3} w}{\partial x^{3}}\right)\right]
$$

where we have used the notations

$$
D=\frac{E h^{3}}{12\left(1-\nu^{2}\right)}, \quad \Upsilon=\frac{\eta h^{3}}{12\left(1-\mu^{2}\right)} .
$$

If we perform notation changes $D \leftrightarrow E I$ and $\Upsilon \leftrightarrow \eta I$ in (5.6), we obtain the corresponding equations for a viscoelastic beam. 
Let us define the parameter $t_{\mathrm{R}}$ as a retardation time constant (see Sobotka 1984)

$$
t_{\mathrm{R}}=\frac{\eta}{E} .
$$

The SI unit of $t_{\mathrm{R}}$ is the second. With the help of (5.8) and assuming that the elastic and viscous Poisson rations coincide, i.e. $\mu=\nu$, we may write

$$
\Upsilon=t_{\mathrm{R}} D
$$

Writing the dynamic equilibrium for the bending forces affecting the panel,

$$
\frac{1}{m} \frac{\partial^{2} M}{\partial x^{2}}+\frac{T_{0}}{m} \frac{\partial^{2} w}{\partial x^{2}}=\frac{\partial^{2} w}{\partial t^{2}}+2 V_{0} \frac{\partial^{2} w}{\partial x \partial t}+V_{0}^{2} \frac{\partial^{2} w}{\partial x^{2}},
$$

and inserting expression (5.6) for the bending moment into (5.9) (see also Ding and Chen 2008), we have

$$
\begin{gathered}
\frac{\partial^{2} w}{\partial t^{2}}+2 V_{0} \frac{\partial^{2} w}{\partial x \partial t}+\frac{t_{\mathrm{R}} D}{m} \frac{\partial^{5} w}{\partial x^{4} \partial t}+\left(V_{0}^{2}-\frac{T_{0}}{m}\right) \frac{\partial^{2} w}{\partial x^{2}} \\
+\frac{D}{m} \frac{\partial^{4} w}{\partial x^{4}}+V_{0} \frac{t_{\mathrm{R}} D}{m} \frac{\partial^{5} w}{\partial x^{5}}=0 .
\end{gathered}
$$

In (5.10), $m$ is mass per unit area, and $T_{0}$ is a constant tension at the panel ends, having the unit of force per unit length. With the change $D \leftrightarrow E I$ (and $\left.t_{\mathrm{R}} E I=\eta I\right)$ in $(5.10)$, one has the dynamic equation for the axially moving viscoelastic beam.

For a derivation of the dynamic equation for a non-linear moving viscoelastic beam based on Newton's second law, refer to Ghayesh (2011). Derivation of the dynamic equation for the axially moving viscoelastic plate, via Hamilton's principle, is given in Tang and Chen 2013. Their equation is reduced into (5.10) when we assume that the displacement $w$ does not vary in the $y$ direction, and that the axial velocity of the plate is constant.

Since (5.10) is of the fifth order in space, five boundary conditions are needed. We first assume that both the ends are clamped and, therefore, we have

$$
w(0, t)=w(\ell, t)=0 \quad \text { and } \quad \frac{\partial w}{\partial x}(0, t)=\frac{\partial w}{\partial x}(\ell, t)=0 .
$$

These boundary conditions can be derived, e.g., by setting clamped boundary conditions for the panel in the reference frame moving with the panel, and performing an appropriate change of variables. For details, see e.g. Chen and Ding (2010). Since the panel is moving in the positive $x$ direction, we seek a fifth condition at the in-flow end $x=0$, indicating that we have more information there than at the out-flow end. 
For the bending moment $M$ in (5.6), we write the following continuity condition (see Flügge 1975)

$$
\lim _{\delta \rightarrow 0} \int_{-\delta}^{+\delta} M \mathrm{~d} x=0
$$

where $M$ is as described in (5.6). Denoting the displacement of the panel in the domain $x<0$ by $w^{-}$, we obtain in the limit $\delta \rightarrow 0$ :

$$
-D \frac{\partial w}{\partial x}+\Upsilon\left(\frac{\partial^{2} w}{\partial x \partial t}+V_{0} \frac{\partial^{2} w}{\partial x^{2}}\right)+D \frac{\partial w^{-}}{\partial x}-\Upsilon\left(\frac{\partial^{2} w^{-}}{\partial x \partial t}+V_{0} \frac{\partial^{2} w^{-}}{\partial x^{2}}\right)=0
$$

at $x=0$. Since

$$
\frac{\partial w}{\partial x}(0, t)=0
$$

and thus also

$$
\frac{\partial^{2} w}{\partial x \partial t}(0, t)=0
$$

by the continuity of the panel (see Flügge 1975) it must hold that

$$
\frac{\partial w^{-}}{\partial x}(0, t)=0
$$

and thus

$$
\frac{\partial^{2} w^{-}}{\partial x \partial t}(0, t)=0
$$

Substituting (5.13-5.16) into (5.12), we obtain

$$
\frac{\partial^{2} w}{\partial x^{2}}(0, t)=\frac{\partial^{2} w^{-}}{\partial x^{2}}(0, t) .
$$

That is, the second derivative of the panel deflections before and after the support must coincide. We choose

$$
\frac{\partial^{2} w^{-}}{\partial x^{2}}=0
$$

and obtain the fifth condition

$$
\frac{\partial^{2} w}{\partial x^{2}}(0, t)=0 .
$$

In the cases of an elastic panel $(\Upsilon=0)$, or a viscoelastic panel where partial time derivative is used instead of the material derivative in the constitutive relations, (5.12) does not produce additional conditions. This is as expected.

In the following, we will treat the problem using two different combinations of boundary conditions. First, a clamped boundary condition at the out-flow end and three conditions at the in-flow end are set 


$$
\begin{gathered}
w(0, t)=0, \quad \frac{\partial w}{\partial x}(0, t)=0, \\
\frac{\partial^{2} w}{\partial x^{2}}(0, t)=0, \\
w(\ell, t)=0, \quad \frac{\partial w}{\partial x}(\ell, t)=0 .
\end{gathered}
$$

Equations (5.20-5.22) will be called the $\mathrm{C}^{+}-\mathrm{C}$ conditions. If the condition (5.21) is removed, we obtain the clamped-clamped (C-C) boundary conditions.

Second, we consider a simply supported condition at the out-flow end and three conditions at the in-flow end:

$$
\begin{gathered}
w(0, t)=0, \quad \frac{\partial w}{\partial x}(0, t)=0, \\
\frac{\partial^{2} w}{\partial x^{2}}(0, t)=0, \\
w(\ell, t)=0, \quad \frac{\partial^{2} w}{\partial x^{2}}(\ell, t)=0 .
\end{gathered}
$$

Equations (5.23-5.25) will be called the $\mathrm{C}^{+}-\mathrm{S}$ conditions. If the condition (5.24) is removed, we obtain the clamped-simply supported (C-S) conditions. The last condition in (5.25) corresponds to zero moment for an elastic panel, but for the viscoelastic panel, it should be considered as a purely kinematical (i.e. displacement-like) condition. Alternatively, if $\Upsilon$ is small, it can be viewed as an approximative condition for the moment given by (5.6).

As was mentioned above, we use three boundary conditions at the in-flow end and two conditions at the out-flow end, indicating that we have more information at the in-flow end. The label $\mathrm{C}^{+}$for the three conditions at $x=0$ (in (5.20-5.22) and (5.23-5.25)) is motivated by the fact that clamping is a stronger condition than being simply supported.

Let us transform the dynamic equation (5.10) into a dimensionless form. We introduce the transformations

$$
x^{\prime}=\frac{x}{\ell}, \quad t^{\prime}=\frac{t}{\tau}, \quad w^{\prime}\left(x^{\prime}, t^{\prime}\right)=\frac{w(x, t)}{h} .
$$

Inserting (5.26) into (5.10), omitting the primes and multiplying by $m \ell^{2} /\left(T_{0} h\right)$, we obtain 


$$
\begin{aligned}
\frac{m \ell^{2}}{\tau^{2} T_{0}} \frac{\partial^{2} w}{\partial t^{2}}+2 & V_{0} \frac{m \ell}{\tau T_{0}} \frac{\partial^{2} w}{\partial x \partial t}+\frac{t_{\mathrm{R}} D}{\tau \ell^{2} T_{0}} \frac{\partial^{5} w}{\partial x^{4} \partial t} \\
& +\left(\frac{V_{0}^{2}}{T_{0} / m}-1\right) \frac{\partial^{2} w}{\partial x^{2}}+\frac{D}{\ell^{2} T_{0}} \frac{\partial^{4} w}{\partial x^{4}}+V_{0} \frac{t_{\mathrm{R}} D}{\ell^{3} T_{0}} \frac{\partial^{5} w}{\partial x^{5}}=0 .
\end{aligned}
$$

Next, let us choose

$$
\tau=\ell \sqrt{\frac{m}{T_{0}}},
$$

as a characteristic time, and introduce the dimensionless problem parameters

$$
c=\frac{V_{0}}{\sqrt{T_{0} / m}}, \quad \alpha=\frac{D}{\ell^{2} T_{0}}, \quad \gamma=\frac{t_{\mathrm{R}}}{\tau}=\frac{\eta}{E} \frac{\sqrt{T_{0}}}{\ell \sqrt{m}},
$$

where $\gamma$ is the dimensionless retardation time. Note that (5.28) is not the only way to choose the characteristic time. For example, in the study by Zhou and Wang (2007) concerning a two-dimensional viscoelastic plate model, the dimensionless delay time was defined in a similar manner, but a different choice was used for the characteristic time.

Inserting (5.29) into (5.27), we have

$$
\begin{gathered}
\frac{\partial^{2} w}{\partial t^{2}}+2 c \frac{\partial^{2} w}{\partial x \partial t}+\gamma \alpha \frac{\partial^{5} w}{\partial x^{4} \partial t}+\left(c^{2}-1\right) \frac{\partial^{2} w}{\partial x^{2}} \\
+\alpha \frac{\partial^{4} w}{\partial x^{4}}+\gamma \alpha c \frac{\partial^{5} w}{\partial x^{5}}=0
\end{gathered}
$$

with the boundary conditions

$$
\begin{gathered}
w(0, t)=\frac{\partial w}{\partial x}(0, t)=\frac{\partial^{2} w}{\partial x^{2}}(0, t)=0, \\
w(1, t)=0, \quad \text { and } \begin{cases}\frac{\partial w}{\partial x}(1, t)=0, & \text { or } \\
\frac{\partial^{2} w}{\partial x^{2}}(1, t) & =0 .\end{cases}
\end{gathered}
$$

Let us represent the solution of the dynamic problem (5.30-5.31), in the following form (using the standard time-harmonic trial function):

$$
w(x, t)=W(x) e^{s t},
$$

where

$$
s=i \omega
$$


and $\omega$ is the characteristic (dimensionless) frequency of small transverse vibrations. As in the previous chapters, considering the system behaviour, the stability exponent $s$ characterizes it in the following manner:

- If the imaginary part of $s$ is non-zero, and

- the real part of $s$ is zero, the panel vibrates harmonically with a small amplitude.

- the real part of $s$ is positive, the amplitude of transverse vibrations grows exponentially (flutter).

- the real part of $s$ is negative, the transverse vibrations are damped exponentially.

- If the imaginary part of $s$ is zero, and

- the real part of $s$ is zero, the panel has a critical point.

- the real part of $s$ is positive, the panel displacement grows exponentially (divergence, buckling).

- the real part of $s$ is negative, the panel displacement decreases exponentially.

The sign of the real part of $s$ characterizes the stability of the panel: if Re $s>0$, the behaviour is unstable, and otherwise it is stable.

Inserting (5.32) into (5.30), we obtain

$$
\begin{gathered}
s^{2} W+s\left(2 c \frac{\partial W}{\partial x}+\gamma \alpha \frac{\partial^{4} W}{\partial x^{4}}\right)+\left(c^{2}-1\right) \frac{\partial^{2} W}{\partial x^{2}}+ \\
\alpha \frac{\partial^{4} W}{\partial x^{4}}+\gamma \alpha c \frac{\partial^{5} W}{\partial x^{5}}=0 .
\end{gathered}
$$

The boundary conditions for $W$ are

$$
\begin{gathered}
W(0)=\frac{\partial W}{\partial x}(0)=\frac{\partial^{2} W}{\partial x^{2}}(0)=0, \\
W(1)=0, \quad \text { and } \begin{cases}\frac{\partial W}{\partial x}(1)=0, & \text { or } \\
\frac{\partial^{2} W}{\partial x^{2}}(1) & =0 .\end{cases}
\end{gathered}
$$

The stability of the travelling viscoelastic panel can be studied by solving $(5.34-5.35)$ for $(s, W)$, parametrized by the transport velocity $c$.

If we neglect the fifth-order derivative in (5.34), and formulate the buckling problem by setting $s=0$ and and removing the (now superfluous) boundary condition 


$$
\frac{\partial^{2} W}{\partial x^{2}}(0)=0
$$

we obtain the buckling problem for an elastic panel (beam), with the boundary conditions $\mathrm{C}-\mathrm{C}$ or $\mathrm{C}-\mathrm{S}$ depending on the chosen boundary condition at the out-flow end.

For an axially moving elastic panel, the critical velocity corresponding to the divergence instability can be found analytically. For a C-C elastic panel, the dimensionless critical velocity is expressed as

$$
c_{\mathrm{cr}}=\sqrt{1+4 \alpha \pi^{2}} \text {. }
$$

For the derivation of (5.36), see, e.g., Wickert and Mote (1990), who derived the formula for travelling elastic beams. The corresponding critical mode is

$$
W(x)=A[1-\cos (2 \pi x)],
$$

where $A$ is an arbitrary constant. Similarly, one may obtain for a C-S elastic panel the following:

$$
c_{\mathrm{cr}}=\sqrt{1+\alpha k_{1}^{2}},
$$

where $k_{1}$ is the smallest positive solution of $\tan k=k$. The critical mode for a C-S elastic panel is

$$
W(x)=A\left[k_{1} \cos \left(k_{1} x\right)-\sin \left(k_{1} x\right)+k_{1} x-k_{1}\right],
$$

where $A$ is an arbitrary constant.

\subsection{Numerical solution}

The problem can be solved using a finite difference approximation. The outline of the finite difference discretization approach is as follows. Our objective is to find $\mathbf{w}=\left(w_{1}, \ldots, w_{n}\right)$ satisfying the discretised form of (5.34-5.35). We will use central differences of second-order asymptotic accuracy:

$$
\begin{gathered}
\frac{\partial w_{j}}{\partial x} \approx \frac{w_{j+1}-w_{j-1}}{2 \Delta x}, \quad \frac{\partial^{2} w_{j}}{\partial x^{2}} \approx \frac{w_{j+1}-2 w_{j}+w_{j-1}}{(\Delta x)^{2}}, \\
\frac{\partial^{4} w_{j}}{\partial x^{4}} \approx \frac{w_{j+2}-4 w_{j+1}+6 w_{j}-4 w_{j-1}+w_{j-2}}{(\Delta x)^{4}}, \\
\frac{\partial^{5} w_{j}}{\partial x^{5}} \approx \frac{w_{j+3}-4 w_{j+2}+5 w_{j+1}-5 w_{j-1}+4 w_{j-2}-w_{j-3}}{2(\Delta x)^{5}} .
\end{gathered}
$$


The interval $[0, \ell]$ is divided to $n+1$ subintervals equal in length, and therefore $\Delta x=1 /(n+1)$. The end points of the subintervals are labelled as

$$
0=x_{0}, x_{1}, x_{2}, \ldots, x_{n}, x_{n+1}=\ell .
$$

We use two virtual points $\left(w_{-2}\right.$ and $\left.w_{-1}\right)$ at the in-flow end and one virtual $\left(w_{n+2}\right)$ point at the out-flow end. From the boundary conditions (5.35), we obtain at the in-flow end the following relations:

$$
\begin{aligned}
& w_{-2}=-w_{2} \quad\left(\text { from } \frac{\partial^{2} W}{\partial x^{2}}(0)=0\right), \\
& w_{-1}=w_{1} \quad\left(\text { from } \frac{\partial W}{\partial x}(0)=0\right) \quad \text { and } \quad w_{0}=0 .
\end{aligned}
$$

Similarly, at the out-flow end:

$$
\begin{aligned}
& w_{n+1}=0, \\
& w_{n+2}= \begin{cases}w_{n}, & (\mathrm{C}), \\
-w_{n}, & (\mathrm{~S}) .\end{cases}
\end{aligned}
$$

To calculate the fifth-order derivative at the out-flow end $(j=n)$, we can use the following backward difference scheme, which provides second-order asymptotic accuracy:

$$
\frac{\partial^{5} w_{j}}{\partial x^{5}} \approx \frac{3 w_{j+2}-16 w_{j+1}+35 w_{j}-40 w_{j-1}+25 w_{j-2}-8 w_{j-3}+w_{j-4}}{2(\Delta x)^{5}} .
$$

We denote the derivative matrices by $\mathbf{K}_{1}, \mathbf{K}_{2}, \mathbf{K}_{4}, \mathbf{K}_{5}$ built up with the help of (5.38) with the following correspondence:

$$
\mathbf{K}_{1}: \frac{\partial W}{\partial x}, \quad \mathbf{K}_{2}: \frac{\partial^{2} W}{\partial x^{2}}, \quad \mathbf{K}_{4}: \frac{\partial^{4} W}{\partial x^{4}}, \quad \mathbf{K}_{5}: \frac{\partial^{5} W}{\partial x^{5}} .
$$

Inserting the matrices $\mathbf{K}_{1}, \mathbf{K}_{2}, \mathbf{K}_{4}, \mathbf{K}_{5}$ into (5.34), we obtain the matrix equation

$$
s^{2} \mathbf{w}+s\left[2 c \mathbf{K}_{1}+\gamma \alpha \mathbf{K}_{4}\right] \mathbf{w}+\left[\left(c^{2}-1\right) \mathbf{K}_{2}+\alpha \mathbf{K}_{4}+\gamma \alpha c \mathbf{K}_{5}\right] \mathbf{w}=0 .
$$

In the case $\alpha=0$ or $c=0$, we obtain a fourth-order equation needing only four boundary conditions. This can be taken into account by choosing the boundary conditions as in (5.40) and (5.41): the virtual point $w_{-2}$ is then needed only by the matrix $\mathbf{K}_{5}$. When $\mathbf{K}_{5}$ is removed from the matrix equation (5.42), the boundary condition

$$
\frac{\partial^{2} W}{\partial x^{2}}(0)=0
$$


is simultaneously removed from the discretised problem. It can be numerically confirmed that when the value of $\alpha$ is decreased, the solution of (5.42) with the boundary conditions $\mathrm{C}^{+}-\mathrm{C}$ approaches the solution of the corresponding elastic problem with the boundary conditions $\mathrm{C}-\mathrm{C}$, and similarly, the $\mathrm{C}^{+}-\mathrm{S}$ solution approaches the elastic C-S solution. This is the case even if we choose

$$
w_{-2}=w_{2} \text { from } \frac{\partial W}{\partial x}(0)=0, \text { and } w_{-1}=-w_{1} \text { from } \frac{\partial^{2} W}{\partial x^{2}}(0)=0 .
$$

The matrix equation (5.42), which is a quadratic eigenvalue problem with respect to $s$, can be rewritten as (see Tisseur and Meerbergen 2001)

$$
\left[\begin{array}{cc}
-\mathbf{M}_{1} & -\mathbf{M}_{0} \\
\mathbf{I} & 0
\end{array}\right]\left[\begin{array}{c}
s \mathbf{w} \\
\mathbf{w}
\end{array}\right]=s\left[\begin{array}{c}
s \mathbf{w} \\
\mathbf{w}
\end{array}\right]
$$

where

$$
\begin{aligned}
& \mathbf{M}_{0}=\left(c^{2}-1\right) \mathbf{K}_{2}+\alpha \mathbf{K}_{4}+\gamma \alpha c \mathbf{K}_{5} \\
& \mathbf{M}_{1}=2 c \mathbf{K}_{1}+\gamma \alpha \mathbf{K}_{4} .
\end{aligned}
$$

The matrix equation (5.43) is now an eigenvalue problem of the standard form

$$
\mathbf{A y}=s \mathbf{y}
$$

with

$$
\mathbf{A}=\left[\begin{array}{cc}
-\mathbf{M}_{1} & -\mathbf{M}_{0} \\
\mathbf{I} & 0
\end{array}\right], \quad \mathbf{y}=\left[\begin{array}{c}
s \mathbf{w} \\
\mathbf{w}
\end{array}\right]
$$

\subsection{Numerical illustrations}

In this section, we consider some numerical examples for the problem (5.345.35 ), using the finite difference discretization presented above. The number of computation points is $n=200$.

Problem parameters fixed to those typical of a paper material and used in the numerical examples are shown in Table 5.1. Paper material constants have been measured for example by Yokoyama and Nakai (2007). Various values are chosen for the retardation time constant $t_{\mathrm{R}}$ in order to demonstrate the effect of viscosity.

Using the physical parameters in Table 5.1, the dimensionless parameter $\alpha$ in (5.29) obtains the value $\alpha=1.8315 \cdot 10^{-7}$. For the retardation time constant $t_{\mathrm{R}}$ and the dimensionless retardation time $\gamma$, we use the values presented in table 5.2.

In Figs. 5.3 - 5.8, the three lowest eigenvalue pairs $s$, see (5.33), are plotted with respect to the dimensionless panel velocity. In the numerical examples, it can be seen that for the parameter values in Table 5.1, the panel behaviour 
Table 5.1 Physical parameters used in the numerical examples.

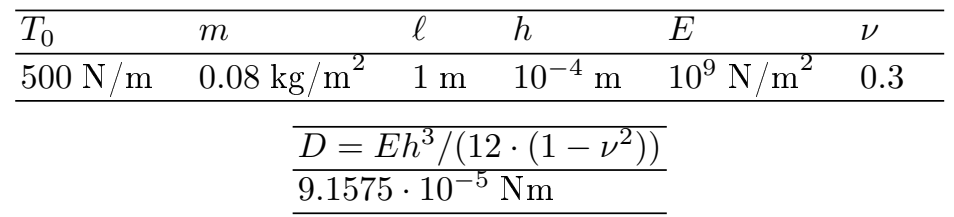

Table 5.2 Chosen values of the retardation time constant $t_{\mathrm{R}}$ and dimensionless retardation time $\gamma$, used in the numerical examples.

\begin{tabular}{llll}
\hline$t_{\mathrm{R}}$ & $5 \cdot 10^{-5} \mathrm{~S}$ & $5 \cdot 10^{-4} \mathrm{~s}$ & $5 \cdot 10^{-3} \mathrm{~s}$ \\
\hline$\gamma$ & $3.953 \cdot 10^{-3}$ & $3.953 \cdot 10^{-2}$ & 0.3953 \\
\hline
\end{tabular}

is stable with harmonic vibrations when the panel velocity $c$ is between 0 and 1 , regardless of the value of the dimensionless retardation time $\gamma$ or the retardation time constant $t_{\mathbf{R}}$. It is also seen that the panel may experience a divergence instability at a critical dimensionless velocity $c_{\mathrm{cr}}$ slightly above the value 1 , depending on the value of $\gamma$. The eigenvalues between 0 and 1 behave similarly in all of the example cases. This behaviour is shown in Figs. $5.4-5.7$, in the upper left corner of each figure.

To illustrate the behaviour close to the possible critical point more closely, the velocity range $1 \ldots 1.00003$ is shown magnified in Figs. 5.3 - 5.8. As found previously for elastic beams (see e.g. Wickert and Mote 1990), at velocities greater than the divergence speed $c_{\mathrm{cr}}$, a flutter instability region may appear. In the present model, it is seen that when the value of the parameter $\alpha$ is increased, the value of the critical velocity increases, and the distance between the possible divergence speed and the possible flutter speed increases.

In Fig. 5.3, the eigenvalue spectra for moving elastic panels are shown with the boundary conditions $\mathrm{C}-\mathrm{C}$ and the boundary conditions $\mathrm{C}-\mathrm{S}$. Figures 5.4 - 5.8 present, in proportion, the eigenvalues spectra for moving viscoelastic panels with three different values of the dimensionless retardation time: $\gamma=$ $3.953 \cdot 10^{-3}, 3.953 \cdot 10^{-2}$, and 0.3953 .

Figures 5.3, 5.4 and 5.5 illustrate that for a panel with small viscosity $\left(\gamma=3.953 \cdot 10^{-3}\right)$, the results are close to those of elastic panels. The values of critical divergence velocities $c_{\mathrm{cr}}$ seem to coincide. See also Table 5.3.

The stable region after the divergence instability region seen in the behaviour of elastic panels in Fig. 5.3 disappears when viscoelasticity is introduced into the model. See Figs. 5.4 and 5.5.

In the case of elastic panels for both types of boundary conditions, the first and second mode couple, representing a coupled-mode flutter. At greater 
Table 5.3 The results for critical velocities. The dimensional critical velocity $\left(V_{0}\right)_{\mathrm{cr}}$ is obtained from $c_{\mathrm{cr}}$ by $(5.29)$ using the parameter values in Table 5.1. (Saksa et al 2012)

\begin{tabular}{llllll}
\hline & & \multicolumn{2}{c}{$c_{\mathrm{cr}}$} & \multicolumn{2}{c}{$\left(V_{0}\right)_{\mathrm{cr}}(\mathrm{m} / \mathrm{s})$} \\
\cline { 3 - 6 }$\gamma$ & $t_{\mathrm{R}}(\mathrm{s})$ & $\mathrm{C}\left({ }^{+}\right)-\mathrm{C}$ & $\mathrm{C}\left({ }^{+}\right)-\mathrm{S}$ & $\mathrm{C}\left({ }^{+}\right)-\mathrm{C}$ & $\mathrm{C}\left({ }^{+}\right)-\mathrm{S}$ \\
\hline 0 & 0 & 1.0000036 & 1.0000018 & 79.0572 & 79.0571 \\
$3.953 \cdot 10^{-3}$ & $5 \cdot 10^{-5}$ & 1.0000036 & 1.0000019 & 79.0572 & 79.0571 \\
$3.953 \cdot 10^{-2}$ & $5 \cdot 10^{-4}$ & 1.0000043 & 1.0000022 & 79.0573 & 79.0571 \\
0.1022 & $1.29 \cdot 10^{-3}$ & 1.0000087 & - & 79.0576 & - \\
0.2183 & $2.76 \cdot 10^{-3}$ & - & 1.0000099 & - & 79.0577 \\
\hline
\end{tabular}

values of velocity and in the case of the $\mathrm{C}-\mathrm{C}$ boundary conditions, the second and the third mode couple, and with the C-S boundary conditions, the first and the third mode couple. All these couplings disappear when viscoelasticity is introduced.

In the upper right corner of Figs. 5.4 and 5.5, the eigenmodes corresponding to the critical velocities $c_{\mathrm{cr}}$ (solid line) and the eigenmodes of corresponding elastic problems (dashed line) are shown. The solutions are very close to each other for elastic problems and viscoelastic problems with a small viscosity. The eigenmodes shown can be found by solving problem (5.34-5.35) with $s=0$, and the critical velocities given by this static analysis and the dynamic analysis are found to be the same.

In Figs. 5.6 and 5.7, the eigenvalue spectra and critical eigenmodes are shown for a dimensionless delay time $\gamma$ ten times greater than in the case analysed above. The changes in the spectra are notable. The values of critical velocities are greater than for the corresponding elastic panels. Also the shapes of the corresponding critical eigenmodes are different. More changes in the spectra can be seen: in the case of $\mathrm{C}^{+}-\mathrm{C}$ boundary conditions, the divergence instability region becomes slightly wider $\gamma=3.953 \cdot 10^{-2}$ than for $\gamma=3.953 \cdot 10^{-3}$. However, the unstable region after the divergence instability region has now become stable, or stated more precisely, the panel now vibrates with a damped amplitude. The second mode has become stable for all values of velocity. In the case of $\mathrm{C}^{+}-\mathrm{S}$ boundary conditions (see Fig. 5.7), the unstable region still exists after the divergence instability, and the second mode is unstable, with divergence-type instability, for some range of velocities greater than $c_{\mathrm{cr}}$.

When the dimensionless retardation time $\gamma$ is further increased, the real part of also the lowest eigenvalue stays negative, and no critical point or loss of instability can be detected. This can be seen in Fig. 5.8. Since no real part 

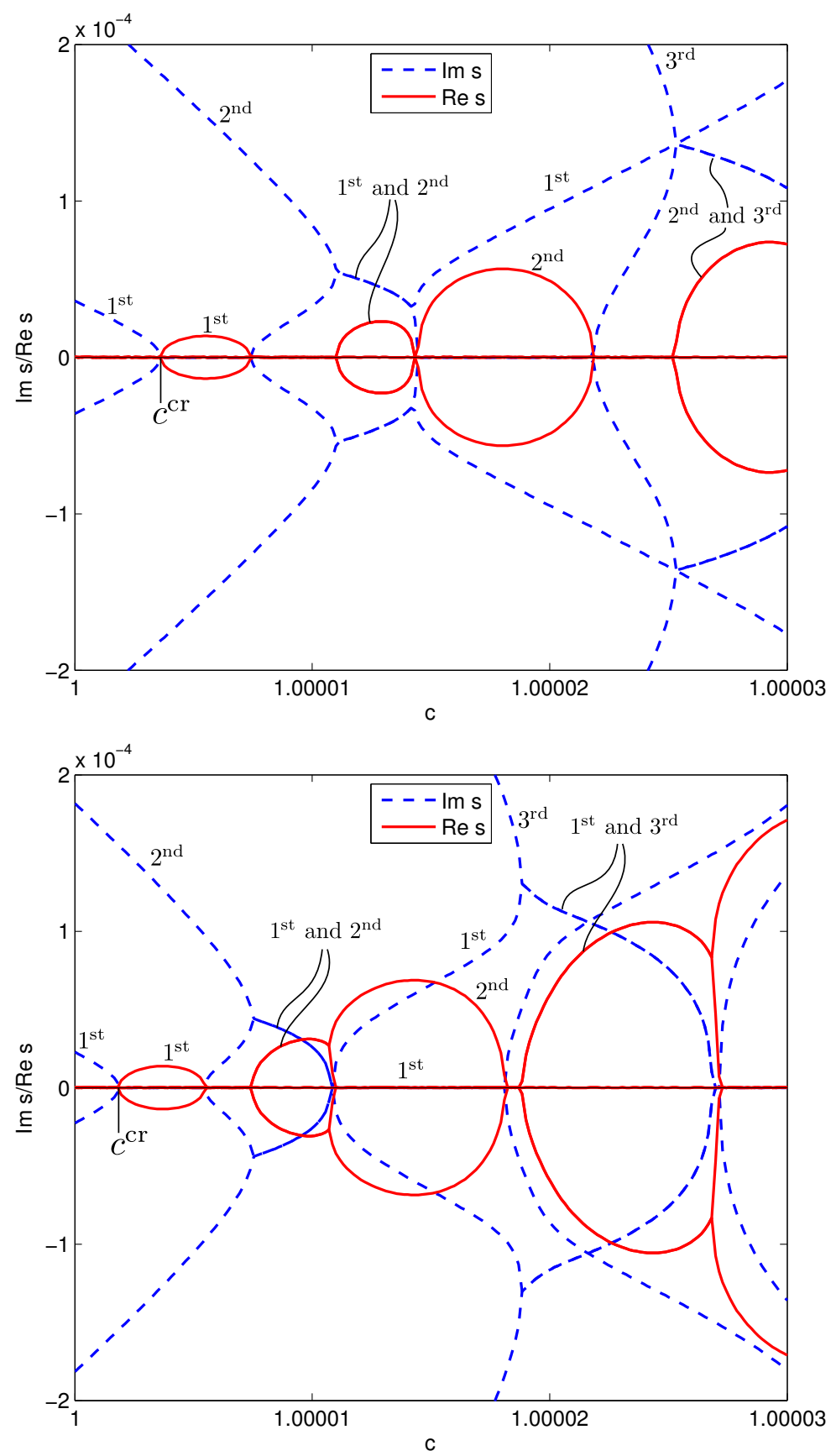

Fig. 5.3 The first three eigenvalue pairs for moving elastic panels $(\gamma=0)$ plotted against the dimensionless velocity $c$. Solid lines present real parts of eigenvalues $(\operatorname{Re} s)$ and dashed lines present imaginary parts $(\operatorname{Im} s)$. The point representing the critical velocity $c_{\mathrm{cr}}$ is labelled. The points representing critical velocities $c_{\mathrm{cr}}$ are labelled. (a) Boundary conditions C-C. (b) Boundary conditions C-S. (Reproduced from Saksa et al 2012) 

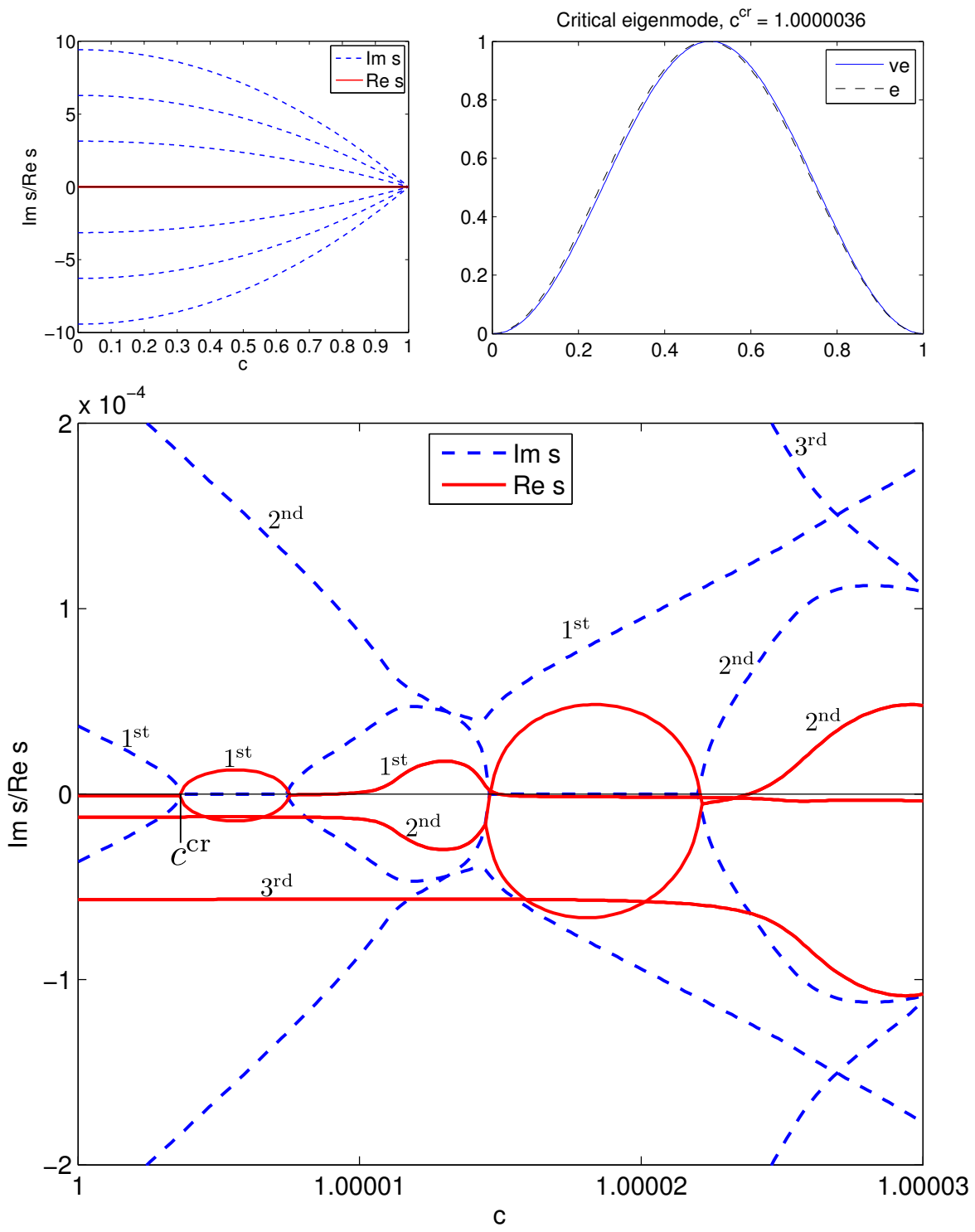

Fig. 5.4 The first three eigenvalue pairs for moving viscoelastic panels with respect to the dimensionless velocity $c$. Dimensionless retardation time $\gamma=3.953 \cdot 10^{-3}\left(t_{\mathrm{R}}=5 \cdot 10^{-5}\right.$ $\mathrm{s}$, almost elastic). Solid lines represent real parts of eigenvalues (Re $s$ ) and dashed lines represent imaginary parts $(\operatorname{Im} s)$. The point representing the critical velocity $c_{\mathrm{cr}}$ is labelled. The behaviour of the eigenvalues $s$ between $c=0$ and $c=1$ is shown at the upper left. The eigenmode corresponding to the (dimensionless) critical velocity $c_{\mathrm{cr}}$ is shown at the upper right. Boundary conditions $\mathrm{C}^{+}$-C. (Reproduced from Saksa et al 2012) 

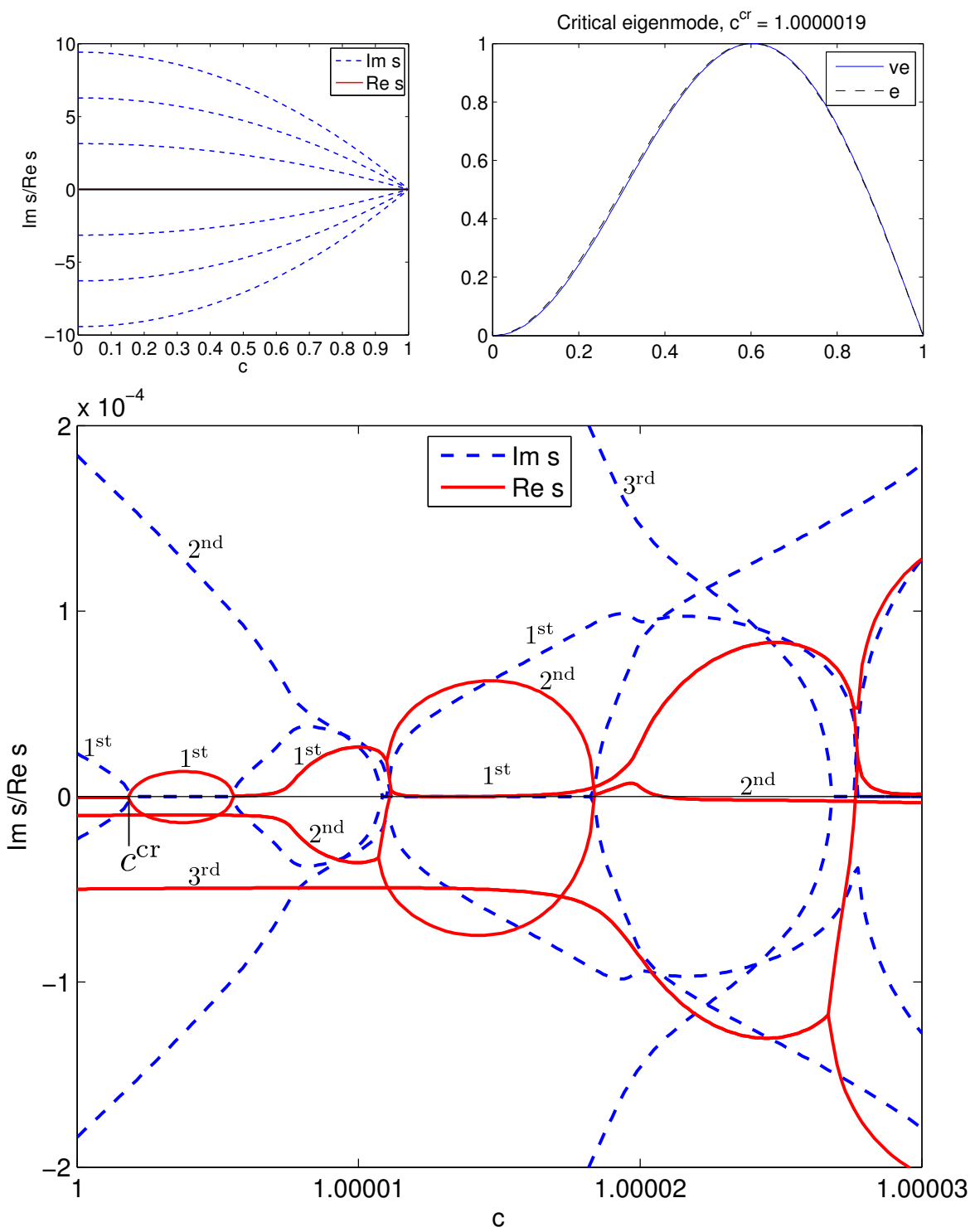

Fig. 5.5 The first three eigenvalue pairs for moving viscoelastic panels with respect to the dimensionless velocity $c$. Dimensionless retardation time $\gamma=3.953 \cdot 10^{-3}\left(t_{\mathrm{R}}=5 \cdot 10^{-5}\right.$ $\mathrm{s}$, almost elastic). Solid lines represent real parts of eigenvalues (Re $s$ ) and dashed lines represent imaginary parts $(\operatorname{Im} s)$. The point representing the critical velocity $c_{\mathrm{cr}}$ is labelled. The behaviour of the eigenvalues $s$ between $c=0$ and $c=1$ is shown at the upper left. The eigenmode corresponding to the (dimensionless) critical velocity $c_{\mathrm{cr}}$ is shown at the upper right. Boundary conditions $\mathrm{C}^{+}-\mathrm{S}$. (Reproduced from Saksa et al 2012) 

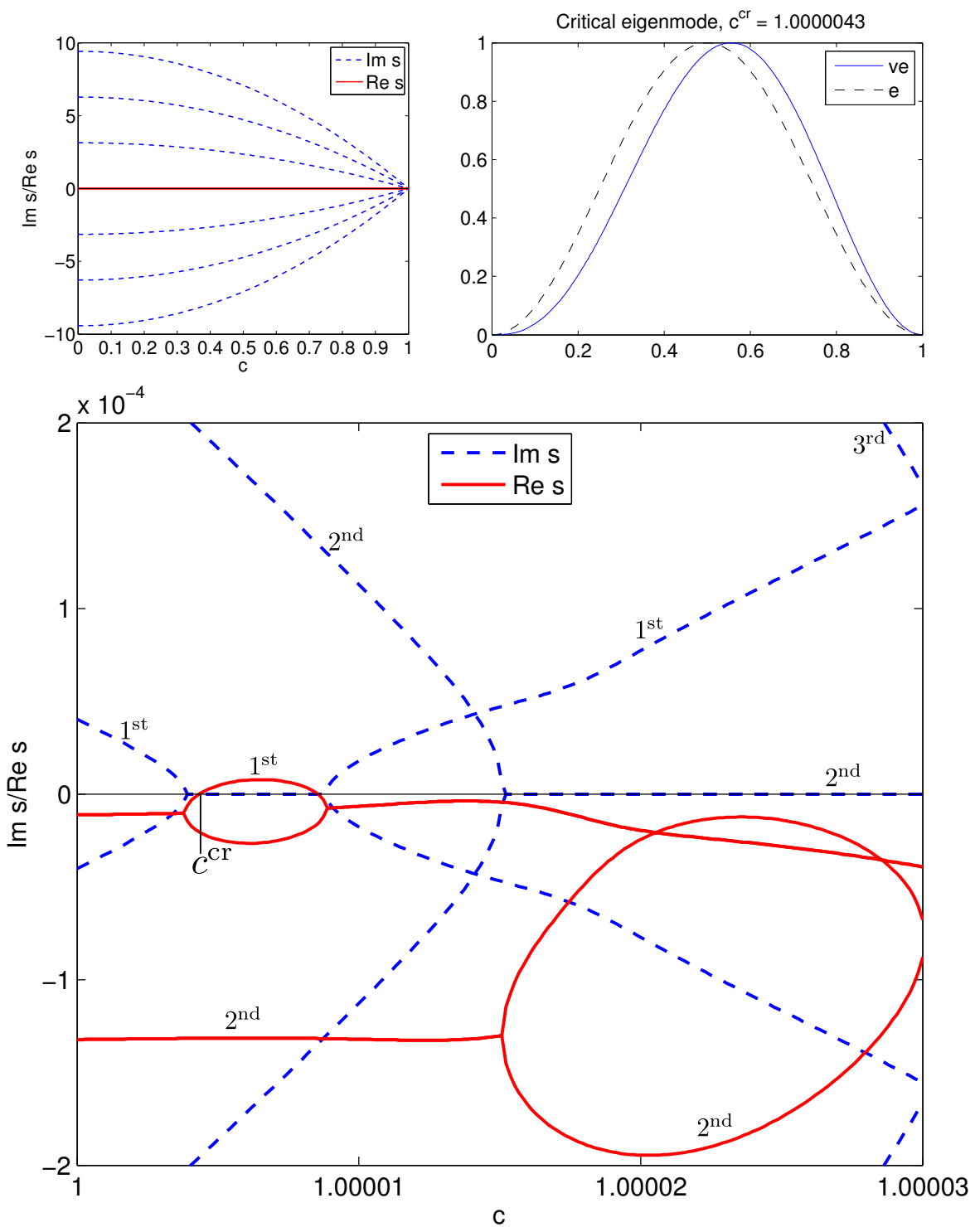

Fig. 5.6 The first three eigenvalue pairs for moving viscoelastic panels with respect to the dimensionless velocity $c$. Dimensionless retardation time $\gamma=3.953 \cdot 10^{-2}\left(t_{\mathrm{R}}=5 \cdot 10^{-4} \mathrm{~s}\right)$. Solid lines represent real parts of eigenvalues $(\operatorname{Re} s)$ and dashed lines represent imaginary parts $(\operatorname{Im} s)$. The point representing the critical velocity $c_{\mathrm{cr}}$ is labelled. The behaviour of the eigenvalues $s$ between $c=0$ and $c=1$ is shown at the upper left. The eigenmode corresponding to the (dimensionless) critical velocity $c_{\mathrm{cr}}$ is shown at the upper right. Boundary conditions $\mathrm{C}^{+}$-C. (Reproduced from Saksa et al 2012) 

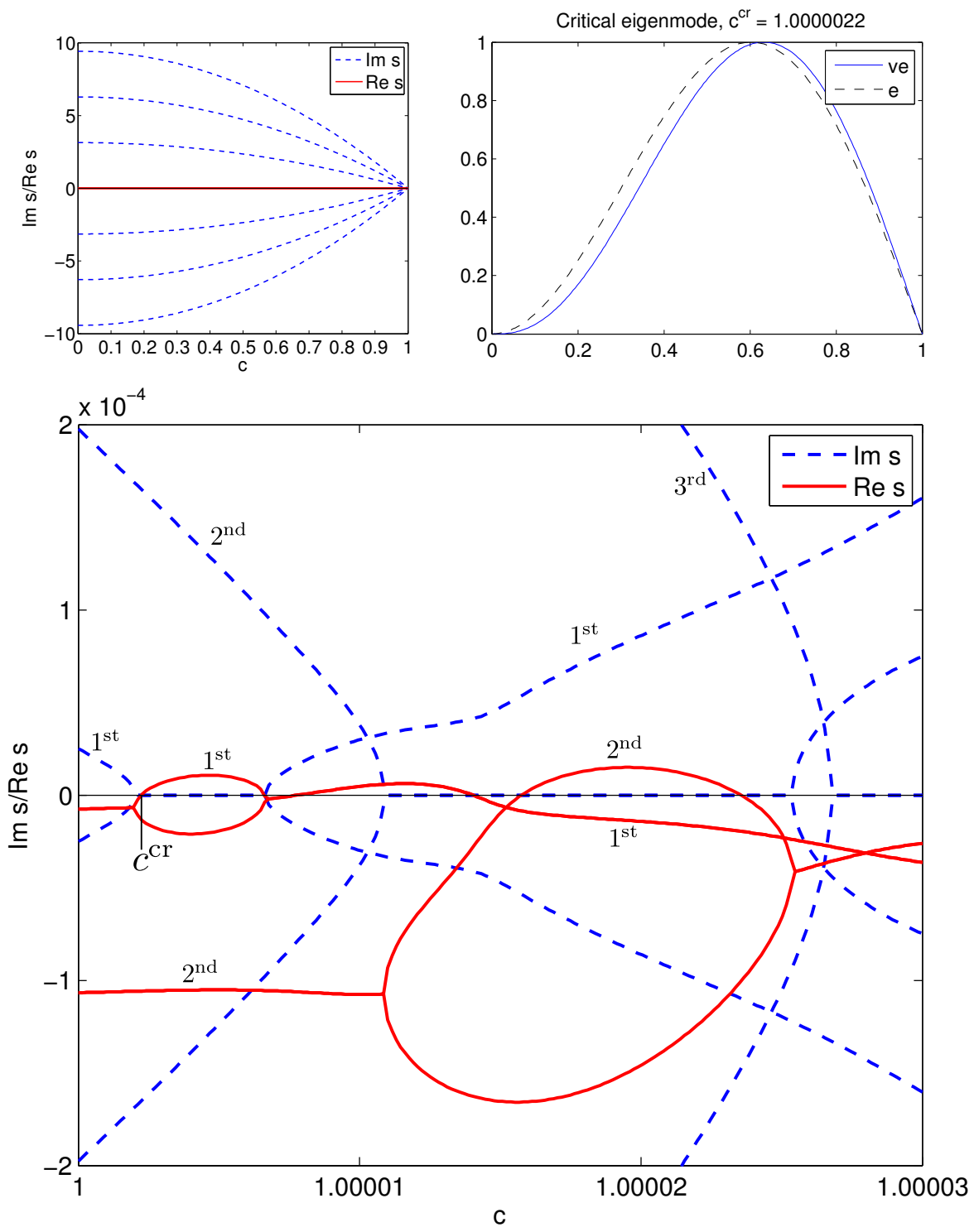

Fig. 5.7 The first three eigenvalue pairs for moving viscoelastic panels with respect to the dimensionless velocity $c$. Dimensionless retardation time $\gamma=3.953 \cdot 10^{-2}\left(t_{\mathrm{R}}=5 \cdot 10^{-4} \mathrm{~s}\right)$. Solid lines represent real parts of eigenvalues ( Re $s$ ) and dashed lines represent imaginary parts $(\operatorname{Im} s)$. The point representing the critical velocity $c_{\mathrm{cr}}$ is labelled. The behaviour of the eigenvalues $s$ between $c=0$ and $c=1$ is shown at the upper left. The eigenmode corresponding to the (dimensionless) critical velocity $c_{\mathrm{cr}}$ is shown at the upper right. Boundary conditions $\mathrm{C}^{+}$-S. (Reproduced from Saksa et al 2012) 

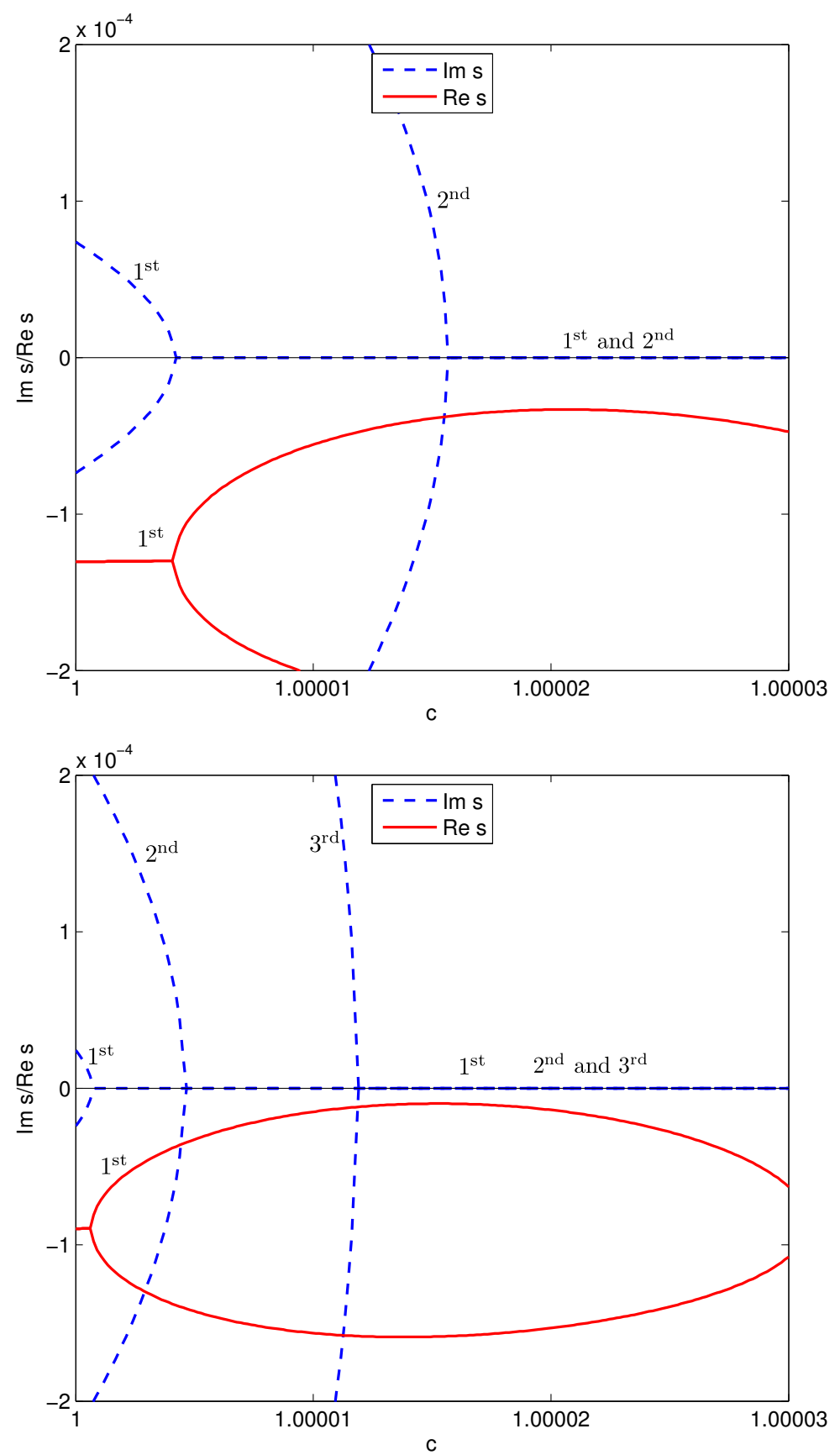

Fig. 5.8 The first three eigenvalue pairs for moving viscoelastic panels with respect to the dimensionless velocity $c$. Dimensionless retardation time $\gamma=0.3953\left(t_{\mathrm{R}}=5 \cdot 10^{-3} \mathrm{~s}\right)$. Solid lines represent real parts of eigenvalues $(\operatorname{Re} s)$ and dashed lines represent imaginary parts $\left(\operatorname{Im} s\right.$ ). (a) Boundary conditions $\mathrm{C}^{+}-\mathrm{C}$. (b) Boundary conditions $\mathrm{C}^{+}-\mathrm{S}$. (Reproduced from Saksa et al 2012) 
of the eigenvalues crosses the $x$ axis, problem (5.34-5.35) with $s=0$ has no solution in such cases. This suggests that high viscosity makes this model stable at any velocity.

The limit values for the dimensionless retardation time $\gamma$ can be found numerically, using e.g. the bisection method. For $\mathrm{C}^{+}-\mathrm{C}$ boundary conditions, after $\gamma \approx 0.1022\left(t_{\mathrm{R}} \approx 1.29 \cdot 10^{-3} \mathrm{~s}\right)$, the real part of the first eigenvalue does not become positive, and also the other (higher) eigenvalues behave similarly. For $\mathrm{C}^{+}-\mathrm{S}$ boundary conditions, the value after which the real part of $s$ remains non-positive is $\gamma \approx 0.2183\left(t_{\mathbf{R}} \approx 2.76 \cdot 10^{-3} \mathrm{~s}\right)$.

Some example values of the critical velocities for different values of dimensionless retardation time $\gamma$ and the corresponding retardation time constant $t_{\mathrm{R}}$ are collected in Table 5.3, including the limit cases. The critical velocities of viscoelastic panels approach the critical velocities of elastic panels as the dimensionless retardation time $\gamma$ approaches zero. The critical velocities for the elastic panels have been calculated analytically with the help of (5.36) and (5.37). The limit value of the dimensionless retardation time $\gamma$ needed for stabilization is higher for the $\mathrm{C}^{+}-\mathrm{S}$ boundary conditions than for the $\mathrm{C}^{+}$ $\mathrm{C}$ conditions, suggesting that the latter case is more stable than the former one. The analytically calculated critical velocities for elastic panels coincide with the numerically calculated critical velocities from the dynamic analysis.

Several studies exist of the model where the partial time derivative is used instead of the material derivative in the viscoelastic relations. We therefore illustrate the eigenvalue spectra of these two different models using the boundary conditions $\mathrm{C}\left({ }^{+}\right)-\mathrm{C}$ and dimensionless retardation time $\gamma=3.953 \cdot 10^{-2}$. The results are shown in Fig. 5.9. The magnitude of $\gamma$ has been found not to qualitatively affect the eigenvalue spectrum in the case of the model with the partial time derivative. The eigenvalue spectrum of this model is very close to the one of the elastic C-C panel (see Fig. 5.3), but the real parts of $s$ are slightly negative before the critical velocity.

The behaviours predicted by the two different models are totally different as shown in Fig. 5.9. The model where the partial time derivative is used predicts a smaller value for the critical velocity than the model with the material derivative. The behaviours at supercritical speeds from the two models differ from each other in many ways. First, after the divergence instability, a second stable region can be seen in the model with the partial time derivative but vibrations of the panel are damped in the model with the material derivative. Secondly, after this, according to the model with the partial time derivative, the panel undergoes couple-mode flutter, but remains stable undergoing damped vibrations according to the model with the material derivative.

In Fig. 5.10, the dynamic behaviour of the viscoelastic panel at subcritical and super-critical velocities is illustrated. For the space discretization, finite differences are used as reported in Sect. 5.2, and the time discretization has been performed via the fourth-order Runge-Kutta method. The initial displacement $w(x, 0)$ has been chosen as the critical eigenmode 


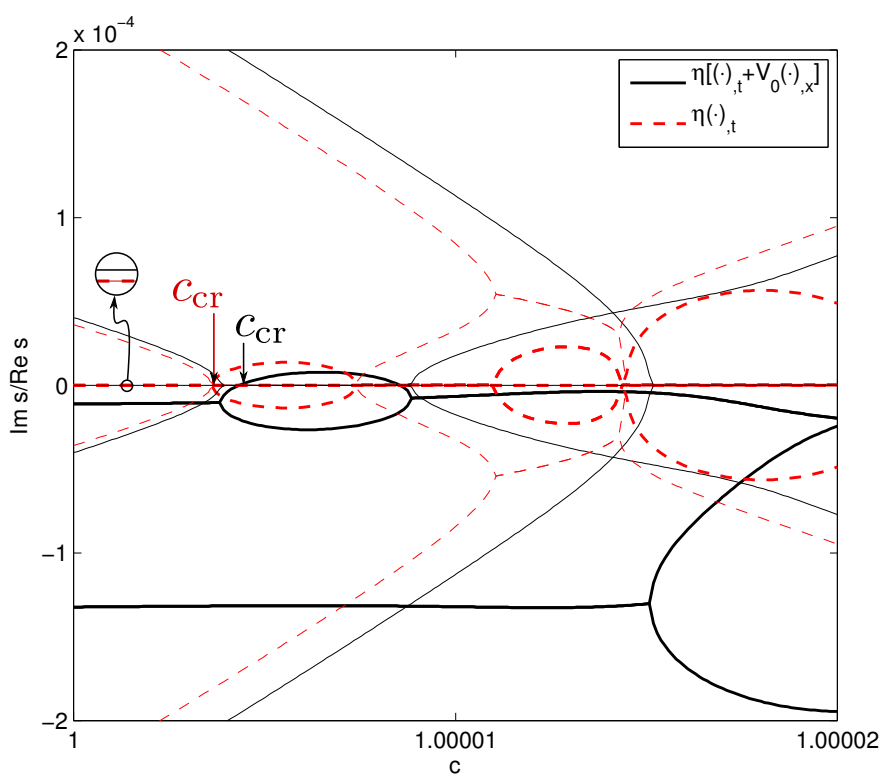

Fig. 5.9 Comparison of two different models. Solid lines show the spectra of the model with the material derivative in viscoelastic relations. Dashed lines represent the model with the partial time derivative in the viscoelastic relations. Real parts of eigenvalues are plotted in bold line, imaginary parts in light line. Boundary conditions $\mathrm{C}\left({ }^{+}\right)-\mathrm{C}$. Dimensionless retardation time $\gamma=3.953 \cdot 10^{-2}\left(t_{\mathrm{R}}=5 \cdot 10^{-4} \mathrm{~s}\right)$. (Reproduced from Saksa et al 2012)

with $\gamma=3.953 \cdot 10^{-2}$ (shown in Fig. 5.6), and $(\partial w / \partial t)(x, 0)=0$ initially. Two different values for the dimensionless retardation time are illustrated, $\gamma=3.953 \cdot 10^{-2}\left(t_{\mathrm{R}}=5 \cdot 10^{-4} \mathrm{~s}\right)$ and $\gamma=0.3953\left(t_{\mathrm{R}}=5 \cdot 10^{-3} \mathrm{~s}\right)$, and the boundary conditions used are $\mathrm{C}^{+}-\mathrm{C}$. With the help of Figs. 5.6 and 5.8, appropriate sub-critical and super-critical velocities have been chosen, being $c=0.99$ (sub-critical) and $c=1.000005$ (super-critical). Figure 5.10 presents the time behaviour of the displacement maxima for the first 10 seconds.

The dynamic behaviour in Fig. (5.10) resembles the behaviour predicted by the dynamic analysis. For $\gamma=0.3953$ at the chosen sub-critical velocity, the panel vibrates with a damped amplitude, and at the super-critical velocity, the panel displacement decreases exponentially (without vibrating). For $\gamma=3.953 \cdot 10^{-2}$ at the super-critical velocity, the displacement grows exponentially and thus the panel is unstable. At the sub-critical velocity, the panel vibrations are damped, but the damping occurs more slowly than for the panel with $\gamma=0.3953$. 

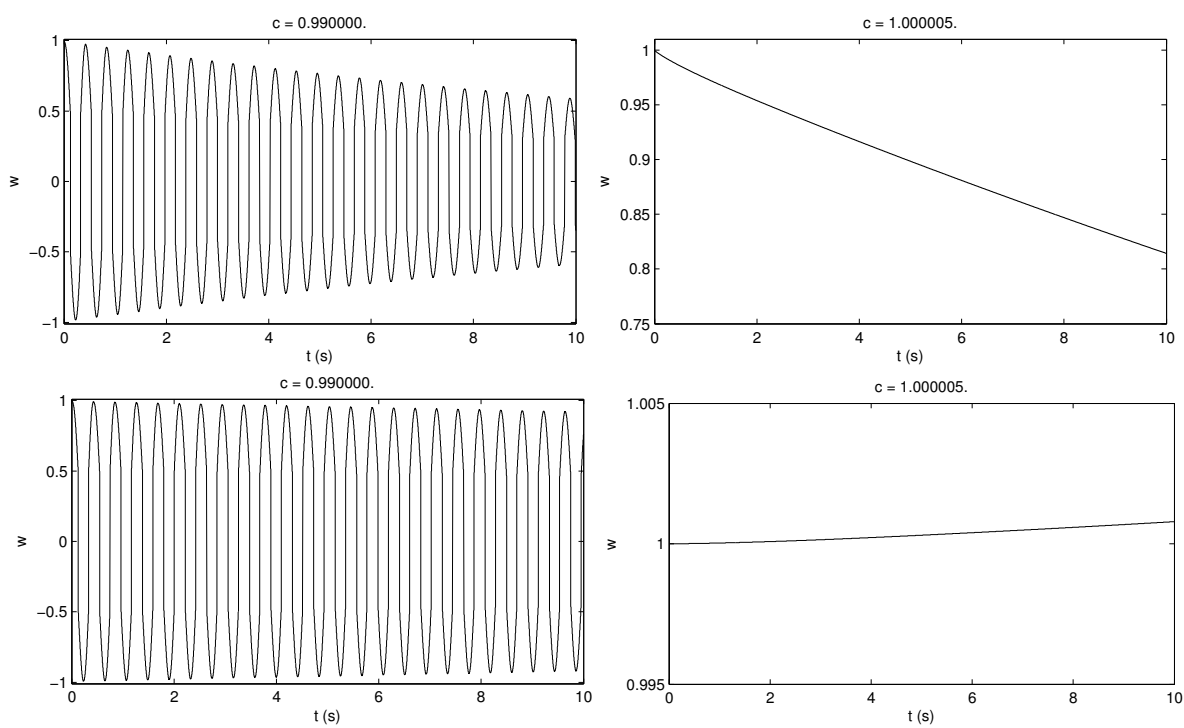

Fig. 5.10 The dynamic behaviour of the displacement maxima $\max _{x \in[0,1]} w(x, t)$ for the first 10 seconds. Boundary conditions $\mathrm{C}^{+} \mathrm{C}$ are used. Top: The dimensionless retardation time is $\gamma=0.3953$. Bottom: $\gamma=3.953 \cdot 10^{-2}$. (Reproduced from Saksa et al 2012)

\section{References}

Alava M, Niskanen K (2006) The physics of paper. Reports on Progress in Physics 69(3):669-723

Bisplinghoff RL, Ashley H (1962) Principles of Aeroelasticity. Dover Publications, Inc., New York, 2nd edition, 1975

Chen LQ, Ding H (2010) Steady-state transverse response in coupled planar vibration of axially moving viscoelastic beams. ASME Journal of Vibrations and Acoustics 132:011,009-1-9, http://dx.doi.org/10.1115/1.4000468

Ding H, Chen LQ (2008) Stability of axially accelerating viscoelastic beams: multi-scale analysis with numerical confirmations. European Journal of Mechanics - A/Solids 27(6):1108 - 1120, DOI: 10.1016/j.euromechsol.2007.11.014

Flügge W (1975) Viscoelasticity, 2nd edn. Springer-Verlag, New York

Ghayesh MH (2011) Nonlinear forced dynamics of an axially moving viscoelastic beam with an internal resonance. International Journal of Mechanical Sciences 53(11):1022-1037, URL http://dx.doi.org/10.1016/j.ijmecsci.2011.08.010

Lee U, Oh H (2005) Dynamics of an axially moving viscoelastic beam subject to axial tension. International Journal of Solids and Structures 42(8):2381 - 2398, URL http://dx.doi.org/10.1016/j.ijsolstr.2004.09.026 
Marynowski K, Kapitaniak T (2002) Kelvin-Voigt versus Bürgers internal damping in modeling of axially moving viscoelastic web. International Journal of Non-Linear Mechanics 37(7):1147 - 1161, DOI: 10.1016/S00207462(01)00142-1

Saksa T, Banichuk N, Jeronen J, Kurki M, Tuovinen T (2012) Dynamic analysis for axially moving viscoelastic panels. International Journal of Solids and Structures 49(23-24):3355-3366, URL http://dx.doi.org/10.1016/j.ijsolstr.2012.07.017

Sobotka Z (1984) Rheology of Materials and Engineering Structures. Elsevier Science Ltd, Amsterdam

Tang YQ, Chen LQ (2013) Stability analysis and numerical confirmation in parametric resonance of axially moving viscoelastic plates with timedependent speed. European Journal of Mechanics / A Solids 37:106-121, URL http://dx.doi.org/10.1016/j.euromechsol.2012.05.010

Timoshenko SP, Woinowsky-Krieger S (1959) Theory of plates and shells, 2nd edn. New York : Tokyo : McGraw-Hill, ISBN 0-07-085820-9

Tisseur F, Meerbergen K (2001) The quadratic eigenvalue problem. SIAM Rev 43:235-286

Wickert JA, Mote CD (1990) Classical vibration analysis of axially moving continua. ASME Journal of Applied Mechanics 57:738-744

Yokoyama T, Nakai K (2007) Evaluation of in-plane orthotropic elastic constants of paper and paperboard. In: 2007 SEM Annual Conference \& Exposition on Experimental and Applied Mechanics

Zhou YF, Wang ZM (2007) Transverse vibration characteristics of axially moving viscoelastic plate. Applied Mathematics and Mechanics (English Edition) 28(2):209-218, URL http://dx.doi.org/10.1007/s10483-007-0209- 


\title{
Chapter 6 Travelling panels interacting with external flow
}

\begin{abstract}
This chapter is devoted to the analysis of the travelling panel, submerged in axially flowing fluid. In order to accurately model the dynamics and stability of a lightweight moving material, the interaction between the material and the surrounding air must be taken into account somehow. The light weight of the material leads to the inertial contribution of the surrounding air to the acceleration of the material becoming significant. In the small displacement regime, the geometry of the vibrating panel is approximately flat, and hence flow separation is unlikely. We will use the model of potential flow for the fluid.

The approach described in this chapter allows for an efficient semianalytical solution, where the fluid flow is solved analytically in terms of the panel displacement function, and then strongly coupled into the partial differential equation describing the panel displacement. The panel displacement, accounting also for the fluid-structure interaction, can then be solved numerically from a single integrodifferential equation.

In the first section of this chapter, we will set up and solve the problem of axial potential flow obstructed by the travelling panel. In the second section, we will use the results to solve the fluid-structure interaction problem, and give some numerical examples.
\end{abstract}

\subsection{Flow problem of surrounding air}

In this section, we will set up and solve analytically the aerodynamic problem for potential flow obstructed by the axially moving panel (see Figs. 6.1-6.3). We will also derive an added-mass approximation from the exact solution, and compare it to some known results in literature.

Consider a travelling panel submerged in potential flow, where the free stream flows toward the right at velocity $v_{\infty}$ (in Euler coordinates). See Fig. 
6.2. The governing equation for the dynamical behaviour of the panel is

$$
m \frac{\partial^{2} w}{\partial t^{2}}+2 m V_{0} \frac{\partial^{2} w}{\partial x \partial t}+\left(m V_{0}^{2}-T\right) \frac{\partial^{2} w}{\partial x^{2}}+D \frac{\partial^{4} w}{\partial x^{4}}=q_{\mathrm{f}}+g,
$$

where $w \equiv w(x, t)$ is the transverse displacement of the panel, $q_{\mathrm{f}} \equiv q_{\mathrm{f}}(w)$ is the aerodynamic reaction pressure, and $g \equiv g(x, t)$ represents external forces inside the domain. The mass per unit area of the panel is $m$. The panel travels axially at the velocity $V_{0}$, being subjected to axial tension $T$ (the unit of which is force per unit length), and its bending rigidity is $D$, given by (2.6) in Sect. 2.1.2 and repeated here for convenience:

$$
D=\frac{E h^{3}}{12\left(1-\nu^{2}\right)} .
$$

Here $h$ is the thickness of the panel, $E$ is the Young's modulus of the panel material, and $\nu$ is its Poisson ratio. The panel is assumed isotropic.

The aerodynamic reaction $q_{\mathrm{f}}$ is unknown, to be solved from the flow model in terms of $w(x, t)$. The aerodynamic reaction describes how the surrounding air pushes back on the panel, in reaction to the panel vibration. The external forces are considered given, and are allowed to vary dynamically, if desired.

For solving the flow problem of the fluid component, we will apply techniques from the aerodynamics of thin aerofoils, constructing a Green's function type solution via complex analysis. More information on thin airfoil theory is available in the books by Ashley and Landahl (1985), Bisplinghoff and Ashley (1962), and Anderson (1985). Especially the first one contains a clear presentation and uses a similar approach.

\subsubsection{Aerodynamic reaction}

In laboratory coordinates, the velocity potential of the disturbed stream is

$$
\Phi(x, z, t)=x v_{\infty}+\varphi(x, z, t),
$$

where the first term is the free-stream potential, and the second term is a disturbance potential due to the obstacle. The Cauchy-Lagrange integral (see, e.g., Sedov 1972, ch. 9) is

$$
\frac{\partial \Phi}{\partial t}+\frac{1}{2}(\nabla \Phi)^{2}+\frac{p}{\rho_{\mathrm{f}}}-\mathcal{U}=f(t)
$$

where we have assumed that the fluid is incompressible. The fluid density $\rho_{\mathrm{f}}$ is assumed constant. The symbol $\mathcal{U}$ represents the scalar potential of the external forces affecting the flow, $F=\nabla \mathcal{U}$. The force potential exists, based 


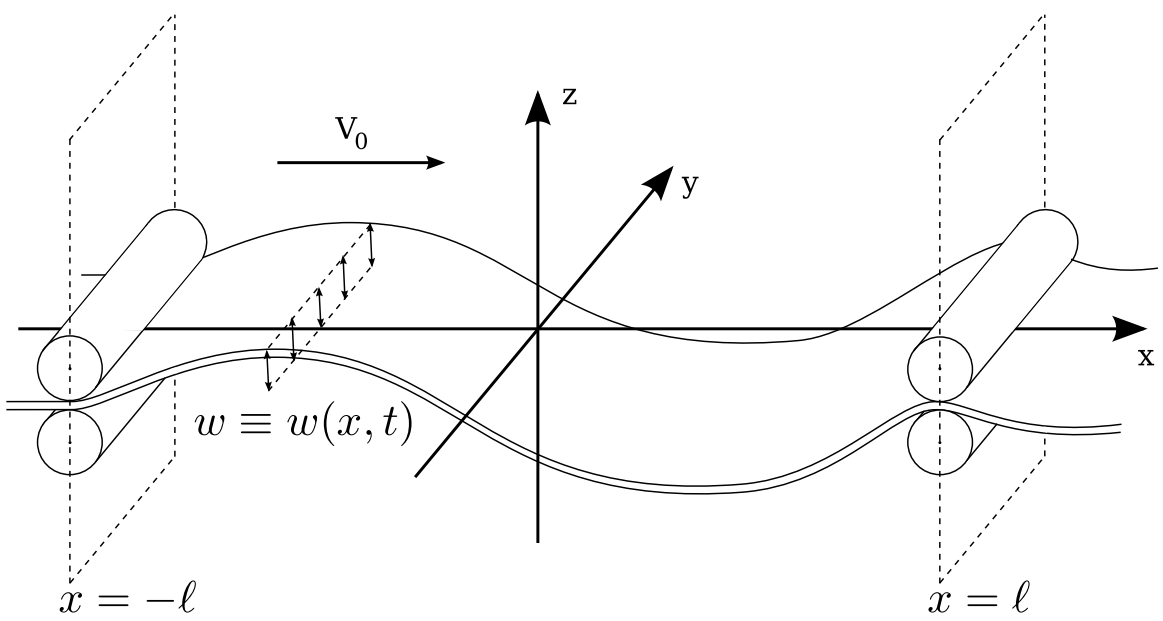

Fig. 6.1 Axially moving thin plate with SFSF boundary conditions, undergoing cylindrical deformation. Two opposite edges are simply supported (S), and the other two edges are free $(\mathrm{F})$. The three-dimensional physical situation.

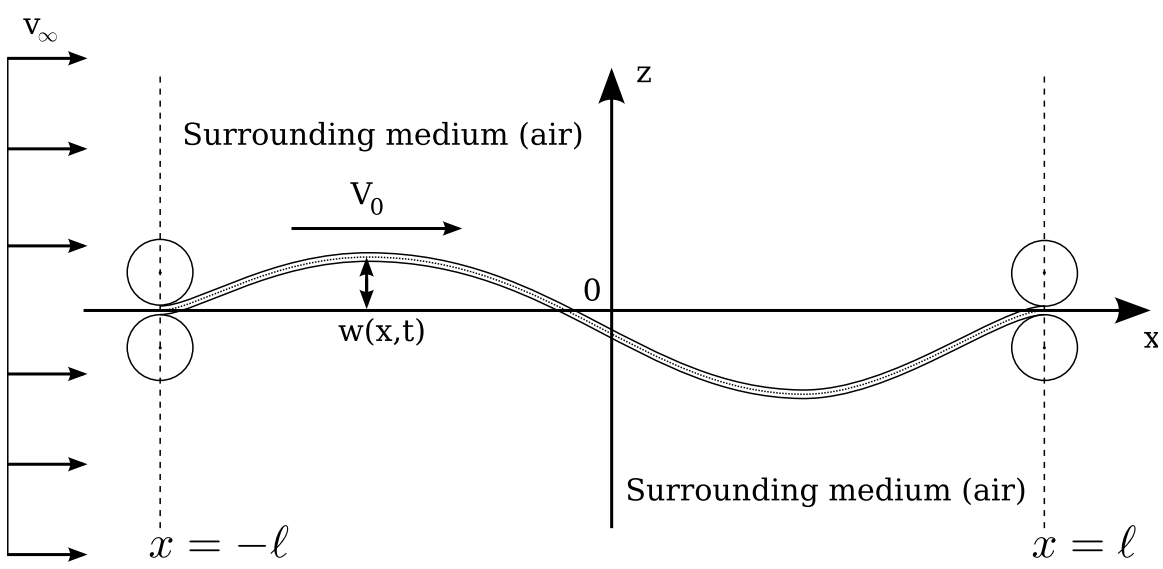

Fig. 6.2 Axially moving panel submerged in axially flowing, two-dimensional ideal fluid. The roller symbols represent simple supports, with presence of axial motion.

on the derivation of the Cauchy-Lagrange integral. The function $f(t)$ is an arbitrary function of time that comes from the integration leading to (6.3).

In the following, let us assume that there are no external forces, i.e. $\mathcal{U} \equiv 0$. Solving (6.3) for $p$, we have

$$
p=\rho_{\mathrm{f}}\left[f(t)-\frac{\partial \Phi}{\partial t}-\frac{1}{2}(\nabla \Phi)^{2}\right] .
$$

The aerodynamic reaction is the pressure difference 


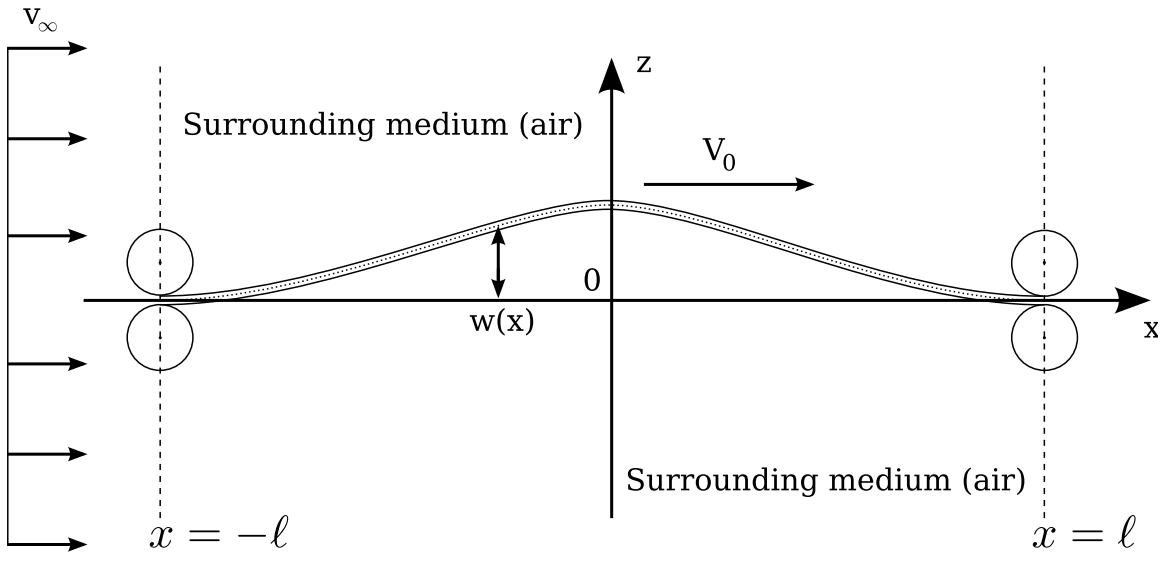

Fig. 6.3 Steady-state problem for the eigenshapes of the axially moving panel submerged in axially flowing, two-dimensional ideal fluid. The roller symbols represent simple supports, with presence of axial motion. Now the panel velocity $V_{0}$ is the eigenvalue, and $w=w(x)$ only (i.e. the panel material undergoes steady-state flow).

$$
q_{\mathrm{f}}(x, t) \equiv p^{-}(x, t)-p^{+}(x, t),
$$

where the superscript notation is defined as

$$
f^{ \pm}(x, t) \equiv \lim _{z \rightarrow 0^{ \pm}} f(x, z, t),
$$

where the upper (respectively lower) signs correspond to each other. That is, the pressure difference is the jump in pressure, caused by the discontinuity introduced by the obstacle through which no flow can occur. The sign convention for (6.5) is that a positive reaction pushes upwards, i.e., toward positive $z$. Obviously, this occurs when the pressure on the lower surface is greater than that on the upper surface.

From (6.2), the gradient of the potential giving the fluid velocity is

$$
\mathbf{v}_{\mathrm{f}} \equiv \nabla \Phi=\left(v_{\infty}+\frac{\partial \varphi}{\partial x}, \frac{\partial \varphi}{\partial z}\right),
$$

where $\mathbf{v}_{f}$ denotes the fluid velocity field. The square of the gradient, needed in (6.4), is

$$
\begin{aligned}
(\nabla \Phi)^{2} & \equiv(\nabla \Phi) \cdot(\nabla \Phi) \\
& =\left(v_{\infty}+\frac{\partial \varphi}{\partial x}\right)^{2}+\left(\frac{\partial \varphi}{\partial z}\right)^{2} \\
& =v_{\infty}^{2}+2 v_{\infty} \frac{\partial \varphi}{\partial x}+\left(\frac{\partial \varphi}{\partial x}\right)^{2}+\left(\frac{\partial \varphi}{\partial z}\right)^{2} .
\end{aligned}
$$


Let us assume that the disturbance velocities $\partial \varphi / \partial x$ and $\partial \varphi / \partial z$ are firstorder small. The linearized squared gradient becomes

$$
(\nabla \Phi)^{2} \approx v_{\infty}^{2}+2 v_{\infty} \frac{\partial \varphi}{\partial x} .
$$

We write (6.5) in terms of (6.4) and insert (6.8):

$$
\begin{aligned}
q_{\mathrm{f}} & =\rho_{\mathrm{f}}\left[\left(\frac{\partial \Phi}{\partial t}\right)^{+}-\left(\frac{\partial \Phi}{\partial t}\right)^{-}+v_{\infty}\left(\frac{\partial \varphi}{\partial x}\right)^{+}-v_{\infty}\left(\frac{\partial \varphi}{\partial x}\right)^{-}\right] \\
& =\rho_{\mathrm{f}}\left(\frac{\partial}{\partial t}+v_{\infty} \frac{\partial}{\partial x}\right)\left(\varphi^{+}-\varphi^{-}\right) .
\end{aligned}
$$

The function $f(t)$ vanishes from this jump expression, because it only depends on time. Also the $v_{\infty}^{2}$ term vanishes, because it is a constant. Finally, we have

$$
\frac{\partial \Phi}{\partial t}=\frac{\partial \varphi}{\partial t}
$$

everywhere, because the chosen free-stream potential $x v_{\infty}$ does not depend on time.

\subsubsection{Slip boundary condition}

We aim to solve the fluid flow in the $(x, z)$ plane region exterior to the panel. On the panel surface, a boundary condition is needed for the fluid flow. The classical choice, which will also be used here, is that flow cannot cross the surface of the panel.

However, we are now allowing axial motion for both the panel and the free stream. Let us work through the derivation of the boundary condition, to illustrate how the axial motion affects it.

We begin with the statement of the boundary condition in terms of the velocity fields in the laboratory (Euler) coordinates,

$$
\mathbf{n} \cdot \mathbf{v}_{\mathbf{f}}=\mathbf{n} \cdot \frac{\mathrm{d} \mathbf{U}}{\mathrm{d} t} \quad \text { on } \Gamma,
$$

where $\mathbf{n}$ is the unit surface normal vector of the panel, $\mathbf{v}_{\mathbf{f}}$ is the fluid velocity field, and $\mathbf{U}$ is the (vector-valued) displacement field of the panel. The symbol $\mathrm{d} / \mathrm{d} t$ denotes the Lagrange derivative (material derivative). The boundary $\Gamma$ is the surface of the panel.

By trigonometry (see Fig. 6.4), we obtain that the local normal vector of the panel surface is

$$
\mathbf{n}=\left(\begin{array}{c}
-\sin \alpha \\
\cos \alpha
\end{array}\right)
$$




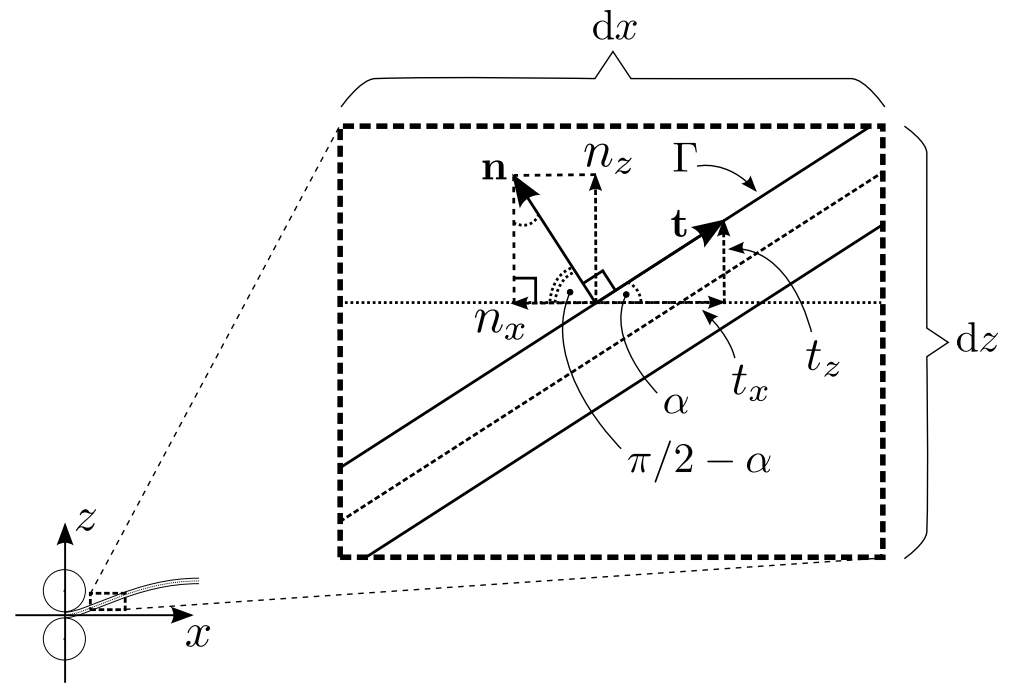

Fig. 6.4 Close-up of the panel surface showing the (local) normal and tangent vectors.

where $\alpha$ is the (counterclockwise) angle between the positive $x$ axis and the local tangent vector of the panel. The direction of $\mathbf{n}$ is chosen such that it points toward positive $z$ when $\alpha=0$. It does not matter which choice we use, as long as we use the same choice for both sides of (6.10).

To find (6.11), consider a differential element of the panel, and in it, the upper $180^{\circ}$ angle between the positive and negative $x$ axis, which is divided by the panel surface and $\mathbf{n}$ into $\alpha$, a straight angle and $(\pi / 2)-\alpha$. Then consider the triangle formed by $\mathbf{n}, n_{z}$ and $n_{x}$, and the angles in this triangle. These must be the same three angles, but in a different order (refer to Fig. $6.4)$.

Alternatively, the normal vector can be found algebraically. Take the positively oriented tangent vector of the panel and the counterclockwise 2D rotation matrix

$$
\mathbf{t}=\left(\begin{array}{c}
\cos \alpha \\
\sin \alpha
\end{array}\right), \quad R(\theta)=\left(\begin{array}{cc}
\cos \theta & -\sin \theta \\
\sin \theta & \cos \theta
\end{array}\right)
$$

Then, evaluate the relation

$$
\mathbf{n}=R(\pi / 2) \mathbf{t}
$$

to obtain (6.11) (refer to Fig. 6.4).

By the definitions of the tangent function, and on the other hand the derivative, we have from the geometry of the situation that

$$
\tan \alpha=\frac{\partial w}{\partial x}
$$


at the limit where $\mathrm{d} z$ and $\mathrm{d} x$ simultaneously tend to zero (refer to Fig. 6.4).

The panel moves axially at a constant velocity $V_{0}$. The displacement field of the panel, written in the Euler coordinates, is

$$
\mathbf{U}(x, t)=\left(V_{0} t+\tilde{u}(\xi(x, t), t), \tilde{w}(\xi(x, t), t)\right),
$$

where $\tilde{u}$ and $\tilde{w}$ are, respectively, the in-plane and out-of-plane displacement functions defined in terms of the Lagrange coordinates, and the $V_{0} t$ term accounts for the global axial motion.

The form of (6.15) is critically important for deriving the slip boundary condition correctly. We have assumed small displacements in (6.15). It should be obvious that in the general case,

$$
\tilde{u}=\tilde{u}(s, t) \text { and } \tilde{w}=\tilde{w}(s, t),
$$

where $s$ is the longitudinal coordinate along the panel. The displacements are, generally speaking, functions of $s$ and not of $\xi$ (or $x$ ). However, in the small displacement regime, we can approximate

$$
s \approx \xi
$$

In addition to being valid for small displacements only, this approximation has a further important mathematical consequence: describing shapes which are not single-valued functions of $\xi$ is impossible in terms of the functions $\tilde{u}(\xi, t)$ and $\tilde{w}(\xi, t)$. This limits the class of shapes that can be described, but the limitation also induces a benefit: self-intersection of the panel surface is automatically prevented without the need for further constraints.

We have defined the displacement functions $\tilde{u}$ and $\tilde{w}$ as being concerned with the elastic behaviour only. Mathematically, they are the solutions of the partial differential equations governing the vibrations of the panel in the longitudinal and out-of-plane directions. This choice implies that if we wrote the displacement field in the Lagrange coordinates, we would have

$$
\tilde{\mathbf{U}}(\xi, t)=(\tilde{u}(\xi, t), \tilde{w}(\xi, t)) .
$$

This is because at any given point of time $t$, each particle is only displaced from its original position in the co-moving coordinate system by the effects of elastic vibration. The Lagrange coordinate system for this problem is defined precisely such that it accounts for the global axial motion. This is why a $V_{0} t$ term appears in (6.15), but not in (6.16).

Finally, it is worth pointing out that the axial tension in a paper machine is in practice generated by using a velocity difference between the rollers at the ends of each free span. This causes the web to stretch, which induces an $x$-dependent longitudinal strain. Typically these strains are small; we have neglected this effect in (6.15). If one wishes to take it into account, one needs a third, $x$-dependent term in the axial component of $\mathbf{U}$. Although this term 
is easier to add into the Euler version $\mathbf{U}$ than the Lagrange version $\tilde{\mathbf{U}}$ (where it will depend on both $\xi$ and $t$ ), from the physics of the situation it is evident that this term, if added, must appear in both coordinate systems.

From (6.15), we obtain in $(x, t)$ coordinates the velocity field of the panel,

$$
\frac{\mathrm{d} \mathbf{U}}{\mathrm{d} t}=\left(V_{0}+V_{0} \frac{\partial u}{\partial x}+\frac{\partial u}{\partial t}, V_{0} \frac{\partial w}{\partial x}+\frac{\partial w}{\partial t}\right) .
$$

For the normal motion of the panel, from (6.11) and (6.17) we obtain the expression

$$
\mathbf{n} \cdot \frac{\mathrm{d} \mathbf{U}}{\mathrm{d} t}=-\sin \alpha\left[V_{0}+\frac{\partial u}{\partial t}+V_{0} \frac{\partial u}{\partial x}\right]+\cos \alpha\left[\frac{\partial w}{\partial t}+V_{0} \frac{\partial w}{\partial x}\right] .
$$

Similarly, from (6.11) and (6.7), for the normal fluid velocity we have

$$
\mathbf{n} \cdot \mathbf{v}_{\mathrm{f}}=-\sin \alpha\left[v_{\infty}+\frac{\partial \varphi}{\partial x}\right]+\cos \alpha \frac{\partial \varphi}{\partial z} .
$$

Subtracting (6.18) from (6.19) and using (6.10) to eliminate the left-hand side gives

$$
-\sin \alpha\left[v_{\infty}-V_{0}-\frac{\partial u}{\partial t}-V_{0} \frac{\partial u}{\partial x}+\frac{\partial \varphi}{\partial x}\right]+\cos \alpha\left[\frac{\partial \varphi}{\partial z}-\frac{\partial w}{\partial t}-V_{0} \frac{\partial w}{\partial x}\right]=0 .
$$

Dividing by $\cos \alpha$, substituting (6.14) for $\tan \alpha$, and multiplying the equation by -1 obtains

$$
\frac{\partial w}{\partial x}\left[v_{\infty}-V_{0}-\frac{\partial u}{\partial t}-V_{0} \frac{\partial u}{\partial x}+\frac{\partial \varphi}{\partial x}\right]-\left[\frac{\partial \varphi}{\partial z}-\frac{\partial w}{\partial t}-V_{0} \frac{\partial w}{\partial x}\right]=0 .
$$

We see that the terms with $V_{0} \partial w / \partial x$ cancel exactly, and all other terms remain. The condition in (6.20) is the exact small-displacement slip boundary condition.

Finally, in small-displacement theory it is customary to assume that the quantities

$$
\frac{\partial w}{\partial x}, \frac{\partial u}{\partial t}, \frac{\partial u}{\partial x}, \text { and } \frac{\partial \varphi}{\partial x}
$$

are small, and discard second-order small terms. This will approximate the panel as perfectly horizontal. This approximation will be used to set up the geometry for the fluid flow problem. It is a useful simplification in order to obtain an analytical solution for the flow problem. Rearranging terms, we obtain the linear approximation

$$
\frac{\partial \varphi}{\partial z}=\frac{\partial w}{\partial t}+v_{\infty} \frac{\partial w}{\partial x} \equiv \gamma(x, t)
$$


which is the approximate small-displacement slip boundary condition on the panel surface. It ensures that the flow will not cross the surface of the panel, accounting for up to first-order small terms.

Equation (6.21) is more convenient to use than (6.20), as it does not require considering the longitudinal displacement $u$ at all. This is another manifestation of the rather general phenomenon that in the small-displacement approximation, the in-plane and out-of-plane components become decoupled from each other.

Note especially that the boundary condition (6.21) does not have a term including $V_{0}$, although in Lagrange coordinates, what the system experiences is indeed the axial velocity difference $v_{\infty}-V_{0}$.

The result is easily confirmed by repeating the steps $(6.15-6.21)$ in the Lagrange coordinates. When doing this, one must be careful to use

$$
\frac{\partial \xi}{\partial t}=-V_{0}
$$

instead of

$$
\frac{\partial x}{\partial t}=V_{0},
$$

as was done in the Euler coordinates. The $V_{0} t$ term, which caused the cancellation, is not present in $\tilde{\mathbf{U}}$, and the velocity coefficient in the corresponding Lagrange boundary condition will indeed be $\left(v_{\infty}-V_{0}\right)$.

On the other hand, the lack of a $V_{0}$ term in (6.21) is not very surprising, since both axial motions are accounted for independently in the Euler coordinate system. Above, the free-stream fluid velocity has been given in the Euler coordinates to begin with. By transforming the mechanics of the panel from the Lagrange into the Euler system, we have written both axial velocities with respect to the same coordinate system. The approach of writing the boundary condition in the Lagrange coordinate system, on the other hand, transforms the fluid velocity into the coordinates axially moving with the panel, introducing a shift by $-V_{0}$ as expected.

\subsubsection{Fluid velocity potential}

As is well-known, velocity potentials fulfill Laplace's equation, as also do harmonic functions in complex analysis. Thus a classical way to approach two-dimensional potential flow problems is to use complex analysis, and seek a harmonic function such that it fulfills the given boundary conditions (see e.g. Ashley and Landahl, 1985).

We identify the complex plane $\mathbb{C}$ with the $(x, z)$ plane in the model,

$$
\eta \equiv x+z i \in \mathbb{C} \quad \Leftrightarrow \quad(x, z) \in \mathbb{R}^{2} .
$$


Because we work in the small deformation regime, we can approximate the panel as a linear cut in the complex plane, located completely on the real axis. The domain of the aerodynamic flow problem is the region exterior to the panel; see Fig. 6.5 .

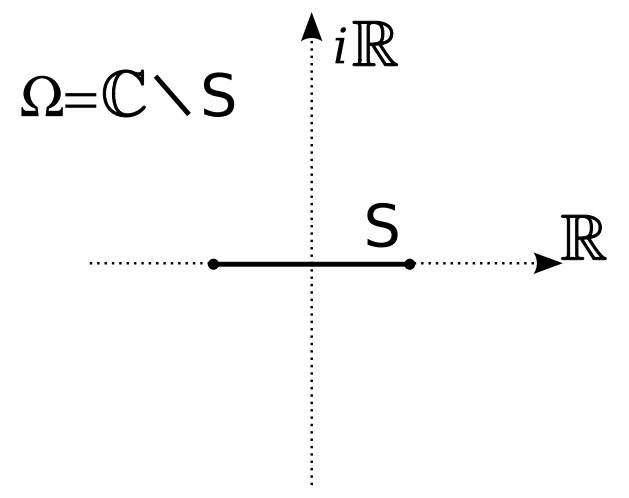

Fig. 6.5 Domain of the problem for the surrounding airflow. The panel is geometrically approximated as the infinitely thin linear cut $S \equiv\{z=0,-1 \leq x \leq 1\}$. The symbol $i$ denotes the imaginary unit, $i \equiv \sqrt{-1}$.

We consider the disturbed flow as a superposition of a constant-velocity free stream and a disturbance term representing the effect of the obstacle. The free-stream potential is linear in $x$; thus it is trivially harmonic. Also, it fulfills the slip condition along all of the real axis. Therefore, we only need to solve the first-order correction to the free stream, i.e. the flow problem for the disturbance potential. This problem can be stated as

$$
\begin{array}{rlrl}
\Delta \varphi \equiv \frac{\partial^{2} \varphi}{\partial x^{2}}+\frac{\partial^{2} \varphi}{\partial z^{2}} & =0 & & \text { in } \Omega \\
\left(\frac{\partial \varphi}{\partial z}\right)^{ \pm} & =\gamma(x, t), & & \text { along } S \\
(\nabla \varphi)_{\infty} & =0, &
\end{array}
$$

where (6.22) is the Laplace equation for the disturbance potential, (6.23) is the linearized slip boundary condition where $\gamma(x, t)$ is given by $(6.21)$, and (6.24) requires that the disturbance in the fluid velocity field vanishes at infinity. The domain $\Omega \equiv \mathbb{C} \backslash S$ (Fig. 6.5). The \pm notation is defined as earlier, (6.6), and the subscript infinity denotes the limit

$$
(\cdot)_{\infty} \equiv \lim _{|\eta| \rightarrow \infty}(\cdot)
$$


where

$$
|\eta| \equiv \sqrt{x^{2}+z^{2}}
$$

is the complex modulus.

Because we have a Laplace problem with only Neumann boundary conditions, the value of the disturbance potential $\varphi$ can only be unique up to an additive constant. This ambiguity can, however, be resolved in an arbitrary manner, because the value of the velocity potential is of no physical interest. To obtain the velocity field, we need only its gradient, on which a global additive constant clearly has no effect.

The doubly connected topology of our fluid domain introduces an additional complication. From potential flow theory, we know that in a doubly connected domain, $\nabla \varphi$ is unique if and only if we prescribe the value of circulation $\Gamma$ around the cut. To illustrate this point, suppose that we have found a (two-dimensional) velocity potential of the noncirculatory flow, $\Gamma=0$, satisfying the slip boundary condition on a given surface $S$. Now, it is possible to add to this flow a simple (inviscid) vortex of arbitrary strength $\Gamma_{0}$, for which one of the circular streamlines has been conformally transformed into the surface $S$. The general mapping theorem (also known as Riemann's mapping theorem; see e.g. Nehari 1952, p. 175) guarantees that this can always be done. By superposition of the two flows, a new irrotational flow has been created, still satisfying the slip boundary condition on $S$, but now the circulation is

$$
\Gamma=\Gamma_{0},
$$

where $\Gamma_{0}$ was arbitrary. Hence, the circulation $\Gamma$ must be specified if we wish to have a unique irrotational flow in a doubly connected two-dimensional domain. (See, e.g., Ashley and Landahl 1985, pp. 42-43.)

This ambiguity is commonly resolved by requiring the Kutta condition (also known as the Kutta-Zhukowski condition) at the trailing edge. The condition picks a unique solution, makes the pressure continuous at the trailing edge, and also moves the trailing stagnation point of the flow to the tip of the airfoil, removing a singularity in the flow velocity field that would otherwise occur there for shapes with sharp trailing edges. See e.g. Ashley and Landahl (1985), and Lighthill (1986). In our solution, we will use a regularity condition by Sherman (1952), which comes from a problem in elastics sharing some of the mathematical form with our flow problem.

It should be pointed out that the problem (6.22-6.24) involves several approximations. Some are obvious, such as the two-dimensional problem setup and the potential flow model. Thus, width-directional variation both in the flow and in the behaviour of the moving web are neglected, as are fluid rotation and viscosity. A further approximation is that the boundary condition (6.23) is only valid up to first-order small terms, as was discussed during the derivation of (6.21).

A more subtle approximation concerns the panel geometry. The domain of the aerodynamic problem is infinite. It consists of the whole $x z$ plane with 
the exception of the cut at

$$
S \equiv\{z=0,-1 \leq x \leq 1\},
$$

which is our linearised representation of the space occupied by the panel (see Fig. 6.5). Thus, although we consider an axially moving panel, for the purposes of this aerodynamic problem the panel only exists on the interval

$$
-1 \leq x \leq 1 .
$$

Effectively, in the panel domain, if we assume $V_{0}>0$, there is a material source at $x=-1$ and a material sink at $x=+1$. Also, the rollers are ignored in the flow problem, being represented only in the boundary conditions for the panel displacement.

The way we have proceeded is of course just one possible choice to build a model for this kind of situation. Alternatively, it could be assumed that the elastic panel is embedded in a rigid baffle of infinite extent, splitting the $x z$ plane into two parts (as in Kornecki et al 1976). This choice leads to a singly connected flow topology.

A third alternative is to model also the rollers and some of the surrounding panel surface, but then a more purely numerical approach is required. Available methods include, for example, the classical vortex panel method (see e.g. Anderson 1985), and the much more general and more modern finite element method (see e.g. Strang and Fix 1973; Ciarlet 1978; Johnson 1987; Allen et al 1988; Krizek and Neittaanmäki 1990; Hughes 2000; Brenner and Scott 2010; and for flow problems specifically, Gresho and Sani 1999; Donea and Huerta 2003).

Finally, although as far as the flow problem is concerned the geometry of the panel is simplified into the straight line segment $S$, the transverse panel velocity $\partial w / \partial t$ and the local angle of the panel with respect to the $x$ axis, $\partial w / \partial x$, are both allowed to be nonzero in the slip boundary condition. Strictly speaking this is of course impossible, but it is a reasonable approximation in the small-deformation range.

The shape and dynamic motion of the surface will affect the boundary condition (6.23), which creates a nonzero disturbance velocity in the fluid along the line segment $S$. This in turn affects the $q_{\mathrm{f}}(w)$ term in the panel equation, feeding back into the motion of the panel. This way the two-way aeroelastic coupling is taken into account. The model works in the smalldisplacement range, i.e., as long as the straight line segment assumption is approximately valid. 


\subsubsection{Complex analysis approach}

Because potential flow is memory-free, the flow field reconfigures itself instantly at each time $t$, independent of its previous history. Therefore, as far as the flow problem is concerned, the time $t$ in the function $\gamma(x, t)$ in boundary condition (6.23) is just a parameter. This is the only time-dependent part in the fluid flow problem (6.22-6.24). Hence, in the following, we will consider an arbitrary fixed value for $t$, and treat only $x$ and $z$ as variables.

In accordance with the complex analysis approach to $2 \mathrm{D}$ potential flows, we introduce an auxiliary analytic function

$$
\Pi(\eta, t)=\Psi(x, z, t)+i \varphi(x, z, t)
$$

of the complex variable $\eta=x+i z$, where $i^{2}=-1$. The Cauchy-Riemann equations and the boundary condition (6.23) for the flow on the panel surface imply that

$$
\left.\frac{\partial \Psi}{\partial x}\right|_{z=0}=\left.\frac{\partial \varphi}{\partial z}\right|_{z=0}=\gamma(x, t)
$$

Let us denote $\Psi(x, t) \equiv \Psi(x, 0, t)$. We have

$$
\Psi(x, t)=\chi(x, t)+C(t),
$$

where

$$
\chi(x, t)=\int_{-1}^{x} \gamma(\xi, t) \mathrm{d} \xi
$$

and $C(t)$ is a real constant of integration for each fixed $t$. Here $\xi$ is a dummy variable for integration. Thus, finding the velocity potential $\varphi$ reduces to the computation of the imaginary part of the analytic function (6.26), whose real part on $[-1,1]$ is $(6.28)$.

In other words, the idea of introducing the auxiliary function $\Pi$ is that we may use the Neumann boundary data (6.23) for the original (unknown) potential $\varphi$ to generate Dirichlet boundary data for the (similarly unknown) stream function $\Psi$, as per (6.28) and (6.29). Note that unlike the usual convention, the real part of $\Pi$ is here the stream function, and the imaginary part is the real-valued potential for which the original problem was formulated.

We use the results given by Sherman (1952) (compare also Ashley and Landahl 1985, chap. 5-3) and represent the solution of this problem as

$$
\Pi(\eta, t)=\frac{1}{2 \pi i}\left(\frac{\eta-1}{\eta+1}\right)^{1 / 2} \int_{-1}^{1}\left(\frac{\xi+1}{\xi-1}\right)^{1 / 2} \frac{\chi(\xi, t)+C(t)}{\xi-\eta} \mathrm{d} \xi
$$

Here also $\xi$ is a dummy variable for integration. The real constant $C(t)$ is determined with the help of the following equation: 


$$
\frac{1}{2 \pi i} \int_{-1}^{1} \frac{\chi(\xi, t)+C(t)}{\sqrt{\xi^{2}-1}} \mathrm{~d} \xi=0,
$$

which represents a regularity condition for the function $\Pi$. The condition comes from Sherman (1952). The (6.31) is obtained by taking the limit $\eta \rightarrow$ -1 in (6.30), and using the identity

$$
(\xi-1)(\xi+1)=\xi^{2}-1
$$

in the denominator. The condition requires that the integral factor in (6.30) vanishes at $\eta=-1$. From condition (6.31), we obtain

$$
C(t)=\frac{1}{\pi i} \int_{-1}^{1} \frac{\chi(\xi, t) \mathrm{d} \xi}{\sqrt{\xi^{2}-1}} .
$$

Using expression (6.32) and the formula (for treatment of a similar integral, see Ashley and Landahl 1985, pp. 94-95),

$$
\frac{1}{2 \pi i} \int_{-1}^{1}\left(\frac{\xi+1}{\xi-1}\right)^{1 / 2} \frac{\mathrm{d} \xi}{\xi-\eta}=\frac{1}{2}\left(\frac{\eta+1}{\eta-1}\right)^{1 / 2}-\frac{1}{2},
$$

we perform substitutions into expression (6.30) and elementary transformations and obtain

$$
\begin{array}{r}
\Pi=\frac{1}{2 \pi i}\left(\frac{\eta-1}{\eta+1}\right)^{1 / 2} \int_{-1}^{1}\left(\frac{\xi+1}{\xi-1}\right)^{1 / 2} \frac{\chi(\xi, t) \mathrm{d} \xi}{\xi-\eta} \\
\quad+\frac{C(t)}{2}\left[1-\left(\frac{\eta-1}{\eta+1}\right)^{1 / 2}\right] \\
=\quad \frac{\sqrt{\eta^{2}-1}}{2 \pi i} \int_{-1}^{1} \frac{\chi(\xi, t) \mathrm{d} \xi}{(\xi-\eta) \sqrt{\xi^{2}-1}}+\frac{C(t)}{2} .
\end{array}
$$

From the representation (6.34), we can compute the quantity $\varphi^{+}$:

$$
\begin{aligned}
\varphi^{+} & =\lim _{z \rightarrow 0^{+}}[\operatorname{Im} \Pi(x+i z)] \\
& =\quad \text { p.v. }\left(-\frac{\sqrt{1-x^{2}}}{2 \pi} \int_{-1}^{1} \frac{\chi(\xi, t) \mathrm{d} \xi}{(\xi-x) \sqrt{1-\xi^{2}}}\right) .
\end{aligned}
$$

Here, we have taken into account that the constant $C(t)$ in (6.34) is real, and consequently must be omitted when the limit of the imaginary part is computed in (6.35). The integration in (6.35) is understood in the sense of Cauchy's principal value, here denoted p.v.(·).

Because the flow is antisymmetric with respect to the linearised plate surface, it holds for the disturbance potential that 


$$
\varphi^{+}-\varphi^{-}=2 \varphi^{+}
$$

See, e.g., Eloy et al (2007) for a similar case. Alternatively, we can take the corresponding limit of (6.34) on the side with negative $z$,

$$
\eta=x-i z \rightarrow x-i \cdot 0\left(z \rightarrow 0^{-}\right)
$$

and obtain the same result.

By definition of Cauchy's principal value, we have

$$
\begin{aligned}
2 \varphi^{+}=\operatorname{p.v} \cdot( & \left.-\frac{1}{\pi} \int_{-1}^{1}\left(\frac{1-x^{2}}{1-\xi^{2}}\right)^{1 / 2} \frac{\chi(\xi, t) \mathrm{d} \xi}{\xi-x}\right) \\
\equiv \lim _{\varepsilon \rightarrow 0}- & \frac{1}{\pi}\left[\int_{-1}^{x-\varepsilon}\left(\frac{1-x^{2}}{1-\xi^{2}}\right)^{1 / 2} \frac{\chi(\xi, t) \mathrm{d} \xi}{\xi-x}\right. \\
& \left.+\int_{x+\varepsilon}^{1}\left(\frac{1-x^{2}}{1-\xi^{2}}\right)^{1 / 2} \frac{\chi(\xi, t) \mathrm{d} \xi}{\xi-x}\right] .
\end{aligned}
$$

Let us integrate by parts and substitute expression (6.29) for $\chi(x, t)$. We have the result

$$
\begin{aligned}
2 \varphi^{+}=\lim _{\varepsilon \rightarrow 0} & {\left[N(x-\varepsilon, x) \int_{-1}^{x-\varepsilon} \gamma(\xi, t) \mathrm{d} \xi\right.} \\
& -N(x+\varepsilon, x) \int_{-1}^{x+\varepsilon} \gamma(\xi, t) \mathrm{d} \xi \\
& -\int_{-1}^{x-\varepsilon} N(\xi, x) \gamma(\xi, t) \mathrm{d} \xi \\
& \left.-\int_{x+\varepsilon}^{1} N(\xi, x) \gamma(\xi, t) \mathrm{d} \xi\right]
\end{aligned}
$$

where we have defined

$$
\begin{aligned}
& N(\xi, x) \equiv \frac{1}{\pi} \ln \left|\frac{1+\Lambda(\xi, x)}{1-\Lambda(\xi, x)}\right|, \quad \text { where } \\
& \Lambda(\xi, x) \equiv\left[\frac{(1-x)(1+\xi)}{(1-\xi)(1+x)}\right]^{1 / 2} .
\end{aligned}
$$

This is obtained by setting 


$$
\frac{\partial u}{\partial \xi}=\frac{\sqrt{\frac{1-x^{2}}{1-\xi^{2}}}}{\xi-x}, \quad v=\chi(\xi, t)
$$

in the integration by parts formula

$$
\int_{a}^{b} \frac{\partial u}{\partial \xi} v \mathrm{~d} \xi=[u v]_{\xi=a}^{b}-\int_{a}^{b} u \frac{\partial v}{\partial \xi} \mathrm{d} \xi
$$

The first term in (6.38) is the boundary term $u v$, evaluated at the upper limit $x-\varepsilon$. The lower limit produces no term, because $\chi(x, t)$ is defined with the help of an integral from -1 to $x$; hence, $\chi(-1, t)=0$. The second term in (6.38) is $u v$, evaluated at the lower limit $x+\varepsilon$. The limits of integration from -1 to $x+\varepsilon$ are due to evaluating $\chi(x+\varepsilon, t)$. Now the upper limit produces no term, because

$$
N(\xi, x) \rightarrow 0 \quad \text { as } \quad \xi \rightarrow 1 .
$$

The last two terms in (6.38) are the straightforward integrated-by-parts terms of the form $u \partial v / \partial \xi$. See Figs. 6.6 and 6.7 for a qualitative illustration of the functions $\Lambda$ and $N$, and Fig. 6.8 for contour plots.

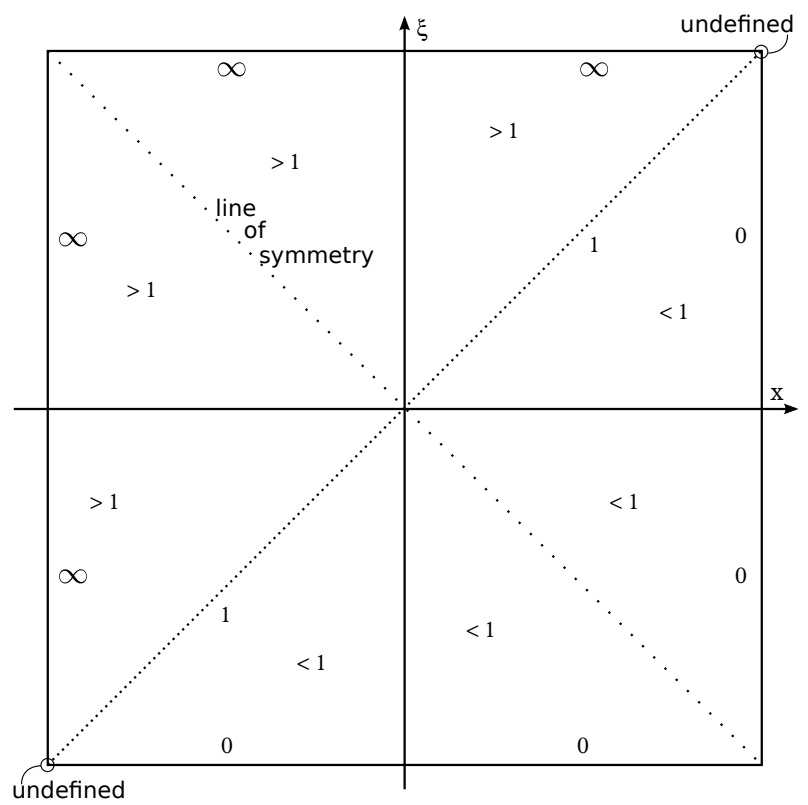

Fig. 6.6 Auxiliary function $\Lambda(\xi, x)$ in $[-1,1] \times[-1,1]$. Qualitative illustration. The infinities should be understood in the sense of limits.

We observe that all terms on the right-hand side of (6.38) are finite; therefore the integration by parts is legitimate. As $\varepsilon \rightarrow 0$, the sum of the first two 


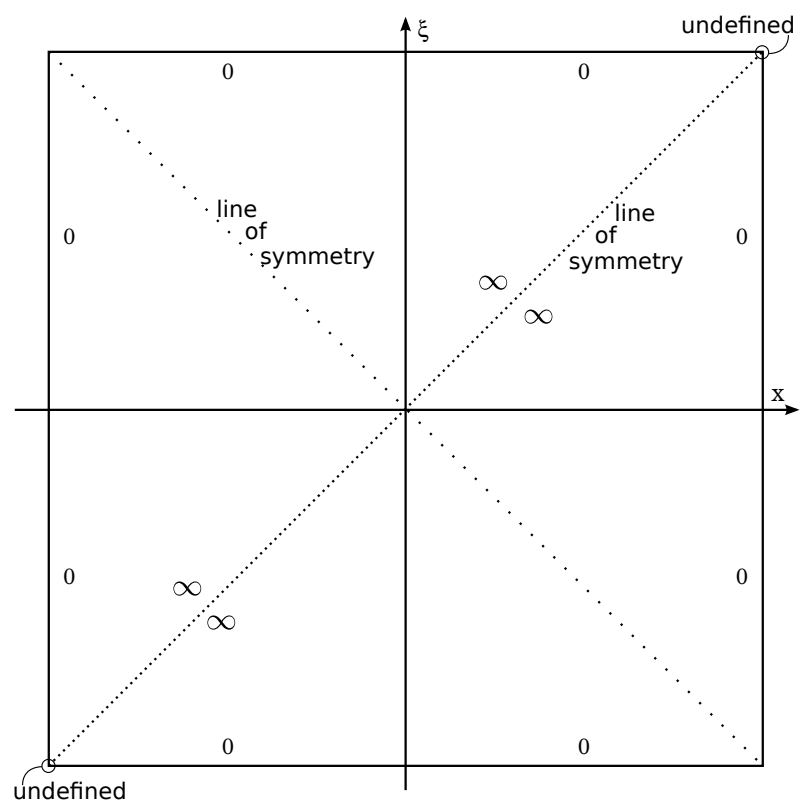

Fig. 6.7 Aerodynamic kernel $N(\xi, x)$ in $[-1,1] \times[-1,1]$. Qualitative illustration. The infinities, and the upper and left edges (which are outside the domain of the auxiliary function $\Lambda(\xi, x)$, needed by $N(\xi, x))$, should be understood in the sense of limits.

terms in (6.38) approaches zero. It can be shown that the last two integrals converge (we will do this below).

Therefore, the required functional dependence is of the form

$$
2 \varphi^{+}(x, t)=-\int_{-1}^{1} N(\xi, x) \gamma(\xi, t) \mathrm{d} \xi .
$$

With the help of (6.9), (6.21), (6.39) and (6.40), we arrive at the expression for the aerodynamic reaction of the fluid. Writing out the coordinate scaling factors $\tau$ and $\ell$ explicitly, we have: 

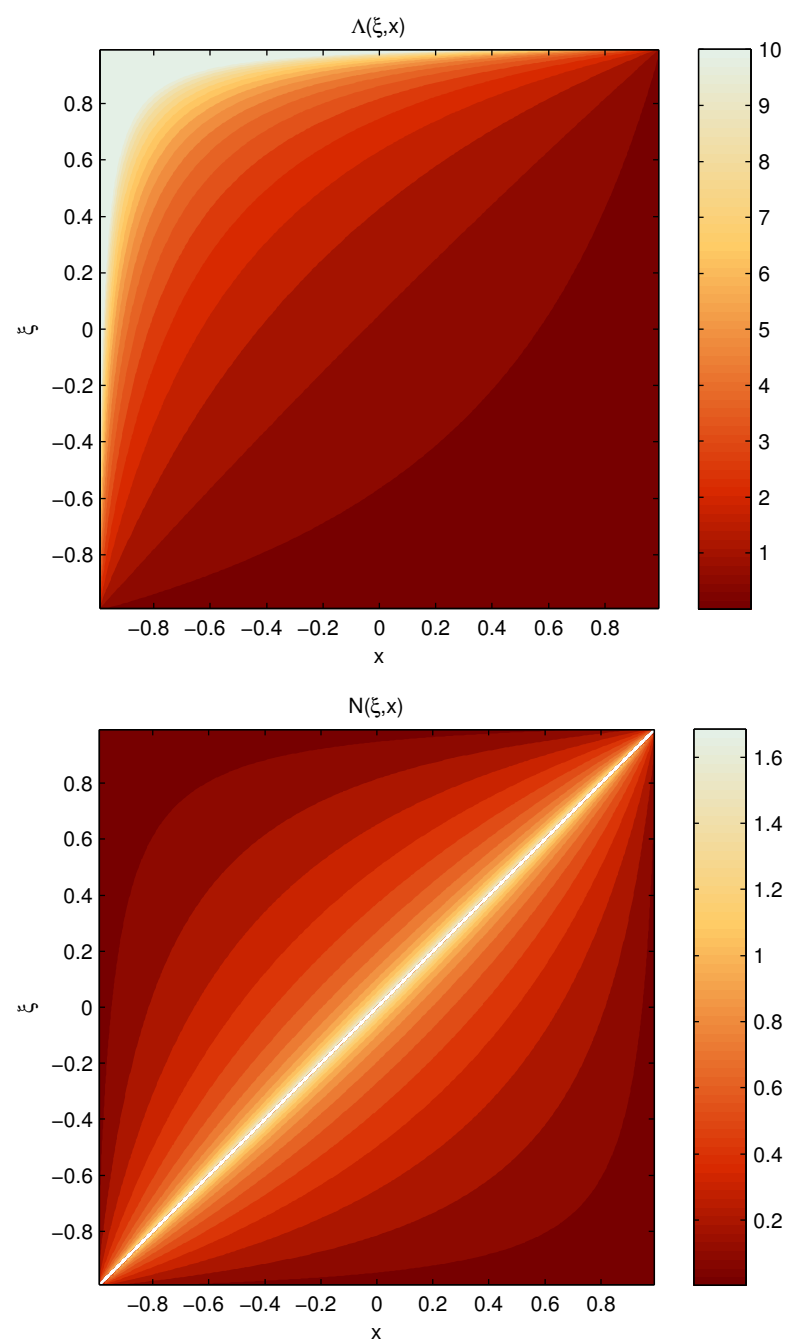

Fig. 6.8 Contour plots of the functions $\Lambda(\xi, x)$ and $N(\xi, x)$. Top: $\Lambda$. Bottom: $N$. Note that both functions grow without bound toward the singularities marked in Figs. 6.6 and 6.7. In both subfigures, the upper end of the colour scale has been chosen arbitrarily to show the structure away from the singularity. 


$$
\begin{aligned}
q_{\mathrm{f}}(x, t) & =p^{-}(x, t)-p^{+}(x, t) \\
& =\rho_{\mathrm{f}}\left(\frac{1}{\tau} \frac{\partial}{\partial t}+\frac{1}{\ell} v_{\infty} \frac{\partial}{\partial x}\right)\left(\varphi^{+}(x, t)-\varphi^{-}(x, t)\right) \\
& =\rho_{\mathrm{f}}\left(\frac{1}{\tau} \frac{\partial}{\partial t}+\frac{1}{\ell} v_{\infty} \frac{\partial}{\partial x}\right)\left(2 \varphi^{+}(x, t)\right) \\
& =-\rho_{\mathrm{f}}\left(\frac{1}{\tau} \frac{\partial}{\partial t}+\frac{1}{\ell} v_{\infty} \frac{\partial}{\partial x}\right) \int_{-1}^{1} N(\xi, x) \gamma(\xi, t) \mathrm{d} \xi \\
& =-\rho_{\mathrm{f}}\left(\frac{1}{\tau} \frac{\partial}{\partial t}+\frac{1}{\ell} v_{\infty} \frac{\partial}{\partial x}\right) \int_{-1}^{1} N(\xi, x)\left(\frac{\ell}{\tau} \frac{\partial}{\partial t}+v_{\infty} \frac{\partial}{\partial x}\right) w(\xi, t) \mathrm{d} \xi \\
& =-\rho_{\mathrm{f}} \frac{1}{\ell}\left(\frac{\ell}{\tau} \frac{\partial}{\partial t}+v_{\infty} \frac{\partial}{\partial x}\right) \int_{-1}^{1} N(\xi, x)\left(\frac{\ell}{\tau} \frac{\partial}{\partial t}+v_{\infty} \frac{\partial}{\partial x}\right) w(\xi, t) \mathrm{d} \xi
\end{aligned}
$$

The scaling factor $\ell$ is the half-length of the span (refer to Fig. 6.2 in Sect. 6.1 ), and $\tau$ is an arbitrary scaling factor for nondimensionalization of the time coordinate. For a physically meaningful scaling, one can choose e.g. $\tau=\ell / C$, where $C=\sqrt{T / m}$ (refer to (6.1) on p. 128). The unit of $\tau$ is $[\tau]=\mathrm{s}$.

The expression (6.41) is valid, because $N(\xi, x)$ is a Green's function of Laplace's equation, and thus, the improper integral (6.40) converges (see, e.g., the book by Evans 1998). The convergence can also be established in a more direct manner, which we will do in the next subsection.

\subsubsection{Properties of aerodynamic kernel}

In this subsection, we will show that the aerodynamic kernel $N(\xi, x)$ is symmetric with respect to reflection by the lines $x= \pm \xi$, and that it is integrable. The symmetricity can be obtained by inspection of (6.39) and algebraic manipulation. We will present it here briefly for completeness.

First, note that the domain of $\Lambda(\xi, x)$ is $(-1,1) \times(-1,1)$, and that of $N(\xi, x)$ is

$$
\{(-1,1) \times(-1,1)\} \backslash\{\xi=x\} .
$$

Consider $\Lambda(\xi, x)$, defined in (6.39). Reflecting the point $(\xi, x)$ with respect to $x=\xi$, let us evaluate $\Lambda(x, \xi)$ :

$$
\begin{aligned}
\Lambda(x, \xi) & =\left[\frac{(1-\xi)(1+x)}{(1-x)(1+\xi)}\right]^{1 / 2} \\
& =\left[\frac{(1-x)(1+\xi)}{(1-\xi)(1+x)}\right]^{-1 / 2}=\frac{1}{\Lambda(\xi, x)} .
\end{aligned}
$$


Then, using (6.42) we have

$$
\begin{aligned}
N(x, \xi) & =\frac{1}{\pi} \ln \left|\frac{1+\frac{1}{\Lambda}}{1-\frac{1}{\Lambda}}\right|=\frac{1}{\pi} \ln \left|\frac{\Lambda+1}{\Lambda-1}\right| \\
& =\frac{1}{\pi} \ln \left|-\frac{1+\Lambda}{1-\Lambda}\right|=\frac{1}{\pi} \ln \left|\frac{1+\Lambda}{1-\Lambda}\right|=N(\xi, x) .
\end{aligned}
$$

Similarly, for reflection with respect to $x=-\xi$, we have

$$
\Lambda(-x,-\xi)=\Lambda(\xi, x),
$$

and thus also

$$
N(-x,-\xi)=N(\xi, x),
$$

because $N$ depends on $\xi$ and $x$ only implicitly via $\Lambda(\xi, x)$. Thus $N$ is symmetric in reflection with respect to the lines $x= \pm \xi$.

Next we will consider the integrability. For the aerodynamic problem, we need to show that the integral (6.40) converges. Let

$$
x \in(-1,1) \text { and } t \in[0, \infty)
$$

be fixed. Let

$$
I_{1}(x) \equiv \int_{-1}^{1} N(\xi, x) f(\xi) \mathrm{d} \xi,
$$

where

$$
f(\xi) \equiv f(\xi ; t),
$$

i.e. $f$ is allowed to depend on $t$, but this dependence is omitted from the notation since we hold $t$ fixed and thus it can be treated as a parameter. We require that $f(\xi)$ is bounded for $-1<\xi<1$.

We estimate (6.44) from above by

$$
\begin{aligned}
I_{1}(x) \leq\left|I_{1}(x)\right| & \equiv\left|\int_{-1}^{1} N(\xi, x) f(\xi) \mathrm{d} \xi\right| \leq \int_{-1}^{1}|N(\xi, x) f(\xi)| \mathrm{d} \xi \\
& \leq \int_{-1}^{1}|N(\xi, x)| \mathrm{d} \xi \cdot \max _{\xi \in[-1,1]}|f(\xi)| .
\end{aligned}
$$

Because

$$
N(\xi, x) \geq 0
$$

over the whole domain, we can omit the absolute value in the integral on the last line above. Thus, it is sufficient to show that the integral 


$$
I_{2}(x) \equiv \int_{-1}^{1} N(\xi, x) \mathrm{d} \xi
$$

converges. Furthermore, as was shown above, the function $N(\xi, x)$ is symmetric with respect to the lines $\xi=x$ and $\xi=-x$. Define

$$
I_{3}(x) \equiv \int_{-1}^{x} N(\xi, x) \mathrm{d} \xi
$$

Due to the symmetries, it holds that

$$
I_{2}(x)=I_{3}(x)+I_{3}(-x) \text { for all } x \in[-1,1] .
$$

See Fig. 6.9 for an illustration. Therefore, it is sufficient to consider the convergence of only the integral (6.47). The integral (6.47) is of the form of the third integral in (6.38), when taken to the limit, so the following argument will prove the convergence of that limit, too. Due to the symmetry, it is also sufficient for proving the convergence of the fourth integral in the same equation.

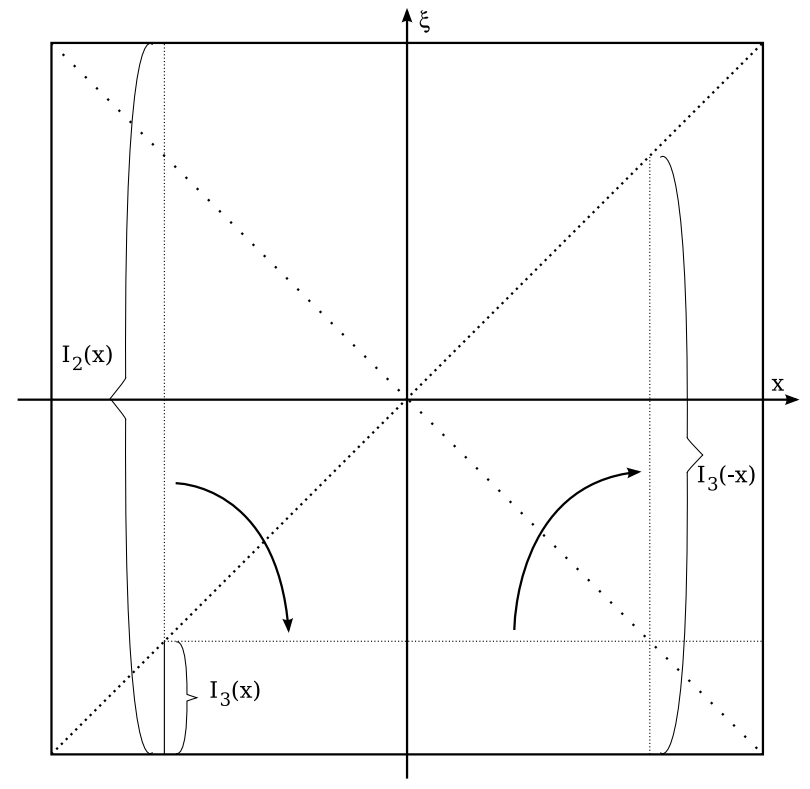

Fig. 6.9 Effect of the symmetries of $N(\xi, x)$ on the integral $I_{2}(x)$.

Now, to show that (6.47) converges, we can use the elementary sandwich theorem, also called dominated convergence, from analysis. The outline of the argument is as follows; details are given in Jeronen (2011). 
Due to the symmetry of $N(\xi, x)$ with respect to $\xi=x$, it is sufficient to consider just one half of the domain. Hence, let $\xi<x$. We leave out strict equality to avoid the singularity of $N(\xi, x)$ at $x=\xi$. Consider the set

$$
D_{1} \equiv\left\{(x, \xi) \in \mathbb{R}^{2} \mid-1<x<1,-1<\xi<x\right\} .
$$

We observe that

$$
0 \leq \Lambda(\xi, x)<1 \quad \text { for all }(x, \xi) \in D_{1} .
$$

Taking this into account, we can estimate $N$ from above, simplifying the expression slightly:

$$
\begin{aligned}
& N(\xi, x) \equiv \frac{1}{\pi} \ln \left|\frac{1+\Lambda}{1-\Lambda}\right| \\
& \stackrel{\text { for } \stackrel{\underline{\xi}}{=} x}{\frac{1}{\pi} \ln \frac{1+\Lambda}{1-\Lambda}} \\
&<\ln \frac{2}{1-\Lambda} \equiv s(\xi, x),
\end{aligned}
$$

where $s(\xi, x)$ is defined as indicated. This simplifies the numerator and gets rid of the absolute value. Let us define transformed coordinates $\eta \equiv x-\xi$ and $\zeta \equiv x+\xi$. Let

$$
r(\eta) \equiv \ln \left(\frac{4}{\eta}\right)
$$

and

$$
r(\xi, x) \equiv r(\eta) \equiv r(x-\xi) .
$$

In the set $D_{1}$, it holds that $\eta \in(0,2)$. In this range, the function $r$ is positive and integrable;

$$
\int r(x-\xi) \mathrm{d} \xi<\infty .
$$

Once we show that on lines parallel with $x=-\xi$, for the function $s$ it holds that

$$
s(\eta, \zeta) \leq s(\eta, 0),
$$

the argument reduces into one dimension. Then, it is sufficient to show that for $\eta \in(0,2)$, we have

$$
s(\eta, 0)<r(\eta) .
$$

Finally, applying the sandwich theorem concludes the argument.

As a final remark to this subsection, we see that $N(\xi, x)$ decreases quickly. Quantitatively speaking, it decreases faster than $\ln (4 / \eta)$, as the distance $\eta \equiv$ $|\xi-x|$ from the singularity $\xi=x$ increases. 


\subsubsection{Added-mass approximation}

Added-mass models are often used for taking into account the inertial effects of fluid-structure interaction in simplified settings. In this section, we will construct an added-mass approximation for our model and compare the result to some existing added-mass models. This is additional material that is will not be used in the rest of the book; for the fluid-structure interaction problem, we will use the original model directly.

Above, it was observed that $N(\xi, x)$ has the following properties:

1. $N$ has a singularity at $\xi=x$ :

$$
N(\xi, x) \rightarrow \infty \text { as } \xi \rightarrow x
$$

2. $N$ is integrable:

$$
\int_{-1}^{1}|N(\xi, x)| \mathrm{d} \xi<\infty, \quad \text { and }
$$

3. the value of $N(\xi, x)$ decreases quickly as the distance from the singularity increases.

Motivated by these properties, let us make the following simplifying approximation:

$$
\int_{-1}^{1} N(\xi, x) f(\xi) \mathrm{d} \xi \approx \mu \int_{-1}^{1} \delta(\xi, x) f(\xi) \mathrm{d} \xi,
$$

where $\delta(\xi, x)$ is the Dirac delta distribution, the function

$$
f(\xi)=f(\xi ; t)
$$

is any admissible function, and $\mu$ is the constant

$$
\mu \equiv \operatorname{mean}_{x \in(-1,1)} \int_{-1}^{1} N(\xi, x) \mathrm{d} \xi=\frac{1}{2} \int_{-1}^{1}\left[\int_{-1}^{1} N(\xi, x) \mathrm{d} \xi\right] \mathrm{dx} .
$$

The symbol mean $(\cdot)$ denotes the average of the indicated quantity over the indicated interval.

The approximated integral in (6.49) is easy to evaluate. Using the definition of the Dirac delta distribution, we have

$$
\mu \int_{-1}^{1} \delta(\xi, x) f(\xi) \mathrm{d} \xi=\mu f(x) \quad \forall x \in[-1,1] .
$$

Numerically, we find that the required mean value is $\mu \approx \pi / 4$. To do this, the formula (6.50) can be evaluated in several ways. One way is to use the Monte Carlo method: take the middle form of the expression (6.50), replace the mean by the sample mean, and sample the integral for e.g. $n=1000$ 
uniformly distributed random values of $x$. The sample mean then gives an approximation for $\mu$.

Alternatively, it is possible to use the rightmost form of (6.50), and evaluate the double integral directly by applying a quadrature method to both the outer and inner integrals. This approach requires many evaluations of the inner integral, and is thus fairly slow, but it gives a more accurate result.

Whichever method is used, due to the singularity of $N(\xi, x)$ at $\xi=x$, in practice the (inner) integral must be numerically approximated as

$$
\int_{-1}^{1} N(\xi, x) \mathrm{d} \xi \approx \int_{-1}^{x-\varepsilon} N(\xi, x) \mathrm{d} \xi+\int_{x+\varepsilon}^{1} N(\xi, x) \mathrm{d} \xi,
$$

where $\varepsilon$ is small.

It is important to notice that the added-mass model, by replacing the exact aerodynamic kernel by the Dirac delta approximation as per (6.49), approximates the fluid-structure interaction as pointwise local. Thus, the added-mass model can be seen as performing mass lumping on the original model; the factor $\mu$ approximates the total strength of the aerodynamic reaction on one point of the panel surface.

Of course, a global mean value is not the only way to perform mass lumping. Alternatively, one can approximate

$$
\int_{-1}^{1} N(\xi, x) f(\xi) \mathrm{d} \xi \approx S(x) \int_{-1}^{1} \delta(\xi, x) f(\xi) \mathrm{d} \xi,
$$

where

$$
S(x) \equiv \int_{-1}^{1} N(\xi, x) \mathrm{d} \xi .
$$

This choice is slightly more sophisticated in that it accounts for differences in the strength of the coupling at different values of $x$. It approximates the effect of the kernel with the local mean at each fixed $x$, instead of the global mean $\pi / 4$. For a plot of $S(x)$, see Fig. 6.10. If fast computation is desired, a polynomial approximation can be used. A least squares fit (by the method of normal equations) using the basis

$$
\phi_{0}(x) \equiv 1, \quad \phi_{1}(x)=x^{2}, \quad \phi_{2}(x)=x^{4}
$$

gives coefficients $c_{0}=0.9892, c_{1}=-0.3195$, and $c_{2}=-0.4872$. This approach leads to a variable-coefficient partial differential equation, and is in spirit similar to the approach of Frondelius et al (2006), where an added-mass model was used, with the added masses taken as functions of $x$ derived from boundary layer theory. (Of course, with the obvious difference that here we use thin airfoil theory instead of boundary layer theory.)

In any case, how accurate the added-mass approximation is depends on the physical situation being considered. In Païdoussis (2008), it is pointed out 


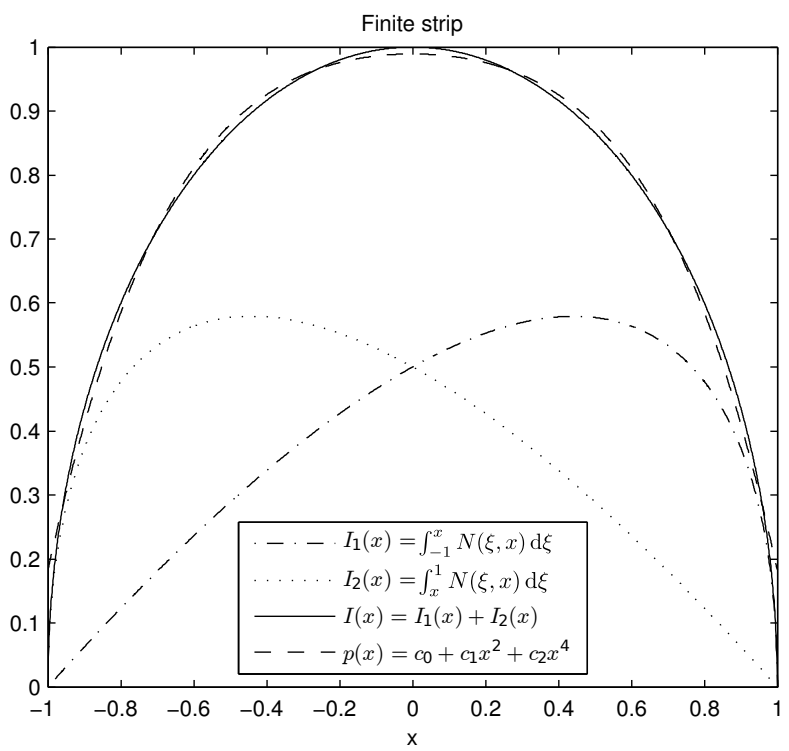

Fig. 6.10 The function $S(x)$ defined by (6.53), for local approximation of the aerodynamic reaction. Both the original function and its three-term polynomial approximation are shown. The polynomial fit coefficients are $c_{0}=0.9892, c_{1}=-0.3195$, and $c_{2}=-0.4872$. Note that the original function touches zero at both ends of the domain, whereas the polynomial approximation does not.

that the problem of plates subjected to axial flow is more complex than the otherwise very similar, canonical problem of the fluid-carrying pipe, exactly due to the nonlocal coupling effect that is ignored by the added-mass model.

Let us work out the added masses predicted by our model. For simplicity, consider only the inertial terms and $q_{\mathrm{f}}$ in (6.1). That is, in (6.1), assume $T=0, D=0$ and $g \equiv 0$ for simplicity, as we can easily add these terms back when finished. We approximate $q_{\mathrm{f}}$ by inserting (6.49) and $\mu=\pi / 4$ into (6.41). Then, we evaluate the approximated aerodynamic integral by (6.51).

In dimensionless coordinates, we have the result

$$
\begin{gathered}
\frac{m}{\tau^{2}} \frac{\partial^{2} w}{\partial t^{2}}+2 \frac{m V_{0}}{\ell \tau} \frac{\partial^{2} w}{\partial x \partial t}+\frac{m V_{0}^{2}}{\ell^{2}} \frac{\partial^{2} w}{\partial x^{2}}= \\
-\rho_{\mathrm{f}} \frac{\pi}{4}\left[\frac{\ell}{\tau^{2}} \frac{\partial^{2} w}{\partial t^{2}}+2 \frac{v_{\infty}}{\tau} \frac{\partial^{2} w}{\partial x \partial t}+\frac{v_{\infty}^{2}}{\ell} \frac{\partial^{2} w}{\partial x^{2}}\right]
\end{gathered}
$$

from which we obtain 


$$
\begin{gathered}
\frac{1}{\tau^{2}}\left[m+m_{\mathrm{a}}\right] \frac{\partial^{2} w}{\partial t^{2}}+2 \frac{V_{0}}{\ell \tau}\left[m+m_{\mathrm{a}} r_{\mathrm{v}}\right] \frac{\partial^{2} w}{\partial x \partial t}+ \\
\frac{V_{0}^{2}}{\ell^{2}}\left[m+m_{\mathrm{a}} r_{\mathrm{v}}^{2}\right] \frac{\partial^{2} w}{\partial x^{2}}=0,
\end{gathered}
$$

where

$$
m_{\mathrm{a}} \equiv \frac{\ell \rho_{\mathrm{f}} \pi}{4} \quad \text { and } \quad r_{\mathrm{v}} \equiv \frac{v_{\infty}}{V_{0}} .
$$

The result reduces to a classical one- or three-term single-parameter addedmass model by choosing $r_{\mathrm{v}}=0$ i.e. $v_{\infty}=0$, no free-stream flow in laboratory coordinates, or $r_{\mathrm{v}}=1$ i.e. $v_{\infty}=V_{0}$, whole air mass moves with the web.

The prediction for the added mass $m_{\mathrm{a}}$ thus derived, in (6.56), agrees with equation (12A) of Pramila (1986):

$$
m_{\mathrm{a}}=\alpha \pi \rho a / 4,
$$

if we take $\alpha=0.5$ in Pramila's equation. Our $\ell$ denotes the span half-length and Pramila's $a$ denotes the full length; hence $a=2 \ell$. The symbol $\rho$ represents the fluid density (the same as our $\rho_{\mathrm{f}}$ ). According to Pramila (1986), the choice $\alpha=0.5$ corresponds to an aspect ratio slightly larger than 1.0, i.e., the span is slightly longer than wide.

Compare also equation (13) of Pramila (1987), due to T. Y.-T. Wu, reported to hold for long and narrow spans:

$$
q_{1}=-\pi \rho\left(b^{2} / 4\right)\left(w_{, t t}+2 v w_{, x t}+v^{2} w_{, x x}\right) .
$$

Here $q_{1}$ denotes the lift force per unit length, and the term is situated on the right-hand side of the dynamical equation, in the same way as our $q_{\mathrm{f}}$. Hence, upon transferring the lift force to the left-hand side of the equation, the sign becomes positive. The velocity $v$ corresponds to our $v_{\infty}$ and the fluid density $\rho$ to our $\rho_{\mathrm{f}}$. When we consider the force per unit surface area, by dividing the given expression by the span width $b$, the corresponding added mass becomes

$$
m_{\mathrm{a}}=\frac{b \rho_{\mathrm{f}} \pi}{4} .
$$

This formulation, instead of $\ell$, uses the span width $b$ as the length scale. Comparing to our approximation in (6.56), the best agreement is obtained when $\ell / b \approx 1$ (i.e. when the span is twice as long as wide).

Finally, in the special case $V_{0}=0$, the effect of fluid cannot be included in the mass only, because for $V_{0}=0$ the quantity $r_{\mathrm{v}}$ in (6.56) becomes undefined. However, the added mass $m_{\mathrm{a}}$ is still well-defined. The added mass $m_{\mathrm{a}}$ and the fluid axial velocity $v_{\infty}$ together fully characterize the approximated effect of the fluid.

In the general case, instead of writing (6.55), we can use the following: 


$$
\begin{gathered}
m_{\text {total }}=m+m_{\mathrm{a}}, \\
p_{\text {total }}=m V_{0}+m_{\mathrm{a}} v_{\infty}, \\
2 K_{\text {total }}=m V_{0}^{2}+m_{\mathrm{a}} v_{\infty}^{2},
\end{gathered}
$$

where $m_{\mathrm{a}}$ is the added mass, $m_{\mathrm{a}} v_{\infty}$ is the added linear momentum, and $\frac{1}{2} m_{\mathrm{a}} v_{\infty}^{2}$ is the added kinetic energy. We have

$$
\frac{1}{\tau^{2}} m_{\text {total }} \frac{\partial^{2} w}{\partial t^{2}}+2 \frac{1}{\ell \tau} p_{\text {total }} \frac{\partial^{2} w}{\partial x \partial t}+\frac{1}{\ell^{2}} 2 K_{\text {total }} \frac{\partial^{2} w}{\partial x^{2}}=0,
$$

or, equivalently,

$$
\begin{gathered}
\frac{1}{\tau^{2}}\left[m+m_{\mathrm{a}}\right] \frac{\partial^{2} w}{\partial t^{2}}+2 \frac{1}{\ell \tau}\left[m V_{0}+m_{\mathrm{a}} v_{\infty}\right] \frac{\partial^{2} w}{\partial x \partial t} \\
+\frac{1}{\ell^{2}}\left[m V_{0}^{2}+m_{\mathrm{a}} v_{\infty}^{2}\right] \frac{\partial^{2} w}{\partial x^{2}}=0 .
\end{gathered}
$$

This generalized added-mass type approximation covers all values of $V_{0}$.

It should be pointed out that due to the forms of (6.55) and (6.60), neither approximation can qualitatively change the dynamic behaviour of the system when compared to the corresponding vacuum case. These added-mass approximations only modify the coefficients of the original constant-coefficient partial differential equation. The partial differential equation itself remains identical to the vacuum case. Thus, the dynamic stability results of the corresponding vacuum case fully qualitatively govern the stability of the addedmass fluid-structure interaction model.

\subsubsection{Notes and discussion}

Above, we set up the aerodynamic problem and derived its solution in a functional form, using the techniques of thin airfoil analysis. We obtained a Green's function solution with respect to the aerodynamic kernel $N(\xi, x)$, determined explicitly in closed form, and to the panel transverse displacement $w(x, t)$. An explicit formula was obtained for the aerodynamic reaction $q_{\mathrm{f}}(w)$. We showed certain symmetry properties and the absolute integrability of the aerodynamic kernel. We also established an analytical upper limit for its values across its whole domain. Finally, we derived a simple addedmass approximation, and suggested a second, more sophisticated added-mass approximation with coefficients depending on $x$.

The assumptions behind the obtained solution are as follows. The fluid is ideal; that is, only potential flow occurs, and fluid rotation and viscosity are neglected. The panel displacement $w$, the fluid disturbance potential $\varphi$, and 
their derivatives are small. Especially, the panel undergoes small deformation only; geometrically, it can be approximated as a straight line segment on the $x$ axis. In the flow problem, only the slip boundary condition depends on the panel's transverse displacement $w$. Although an axially moving panel is studied, in the fluid flow problem the panel only exists in the interval $x \in[-1,1]$. Thus, in the panel domain, there is a material source at $x=-1$ and a material sink at $x=+1$ for positive $V_{0}$. The rollers supporting the panel, and any surrounding parts of the panel itself, are neglected in the flow analysis.

The approach is thus highly approximate, but it is relatively simple and it accounts for two-way coupling between the panel and fluid components of the fluid-stucture interaction model. The deformation of the panel disturbs the fluid, which then causes pressure on the panel surface. The linearity of the coupled model is preserved, because potential flow is memory-free.

Finally, the described solution for the aerodynamic problem does not require the simply supported boundary conditions for the panel that are used in this book. Any choice of boundary conditions for the panel can be used, as long as the panel displacement $w$ and its derivatives inside the domain remain small. For one example, the same aerodynamic reaction is applicable also with the nonsymmetric boundary conditions introduced by Garziera and Amabili (2000), where the case of tape winding onto a reel was investigated. In the paper making context, those boundary conditions could be used for modelling the end of the paper machine, where the finished product is wound onto a reel.

\subsection{Behaviour of travelling panels submerged in ideal fluid}

In this section, we will take the solution of the flow problem derived above, and use it for analyzing the axially moving panel submerged in axial potential flow. The analysis has been split into several subsections to facilitate logical organization. In this first, short section, we set up three problems for the travelling panel submerged in ideal fluid. Then we will perform the space discretization to obtain the semidiscrete form. Much of the work in the space discretization is identical in the steady-state and dynamic cases, so we will perform it only once.

The three problems that will be treated are the static stability problem, the dynamical behaviour problem, and the eigenfrequency problem. We will analyze them starting from the semidiscrete form. The presentation of problemspecific numerical approaches is deferred until the handling of each problem.

Let us begin by defining some common notation for all the problems. Let

$$
C \equiv \sqrt{T / m},
$$


which is the critical velocity of a membrane in vacuum (see, e.g., Chang and Moretti 1991). Define also the dimensionless quantities

$$
\begin{aligned}
\gamma & \equiv \ell \frac{\rho_{\mathrm{f}}}{m}, \\
\beta & \equiv \frac{D}{m C^{2} \ell^{2}}=\frac{D}{\ell^{2} T}, \\
c & \equiv \frac{V_{0}}{C}, \\
\theta & \equiv \frac{v_{\infty}}{C}, \\
\alpha & \equiv \frac{\ell / \tau}{C} .
\end{aligned}
$$

These denote a fluid effect coefficient, dimensionless bending rigidity, dimensionless axial velocities for the panel and the fluid, and dynamic time scale, respectively. The quantities $(6.62-6.65)$ appear in both steady-state and dynamic cases; the quantity (6.66) only in the dynamic case.

All three problems that will be analyzed can be expressed in abstract integro-differential form as

$$
\mathcal{L}\left(w ; V_{0}\right)+\gamma \mathcal{K}\left(w ; v_{\infty}\right)=\mathcal{G},
$$

where $\mathcal{L}$ is the differential operator of the corresponding vacuum problem, $\gamma$ is the fluid effect coefficient, $\mathcal{K}$ is the integro-differential operator accounting for fluid-structure interaction, and $\mathcal{G}=\mathcal{G}(x, t)$ is the (optional) external load function, which does not depend on $w$. The operator $\mathcal{L}$ has the panel velocity $V_{0}$ as parameter, while $\mathcal{K}$ has the fluid free-stream velocity $v_{\infty}$. As the dynamical behaviour problem is the most general of those considered, equation (6.74), further below, can be used as a definition for $\mathcal{L}, \mathcal{K}$ and $\mathcal{G}$.

The first problem is the static stability problem. We use the Euler approach of determining nontrivial steady-state solutions, and the associated critical values of the problem parameter of interest. We will concentrate on finding the critical velocities, which play the role analogous to Euler's critical compression force for the axially compressed beam.

Recall (6.1), which describes the dynamical behaviour of the moving panel submerged in potential flow. Setting the time-dependent terms to zero, and assuming zero external forces $(g \equiv 0)$, we obtain the steady-state equation

$$
\left(m V_{0}^{2}-T\right) \frac{\partial^{2} w}{\partial x^{2}}+D \frac{\partial^{4} w}{\partial x^{4}}=q_{\mathrm{f}},
$$

which describes the buckling of a travelling panel submerged in potential flow. Refer to Fig. 6.3 in Sect. 6.1.

It is convenient to transform the problem into a nondimensional form for analysis. This should be done before we proceed any further, because the aerodynamic reaction, which we wish to insert here, was derived in dimen- 
sionless coordinates. Let us use the dimensionless coordinate

$$
x^{\prime}=x / \ell,
$$

hence each differentiation will produce a factor of $1 / \ell$. Since we will be considering the eigenvalue problem only, the scaling of $w$ is of no concern. Let us choose the dimensionless displacement

$$
w^{\prime}\left(x^{\prime}, t^{\prime}\right) \equiv w(x, t)
$$

where the scaling factor is unity.

In dimensionless coordinates, we have

$$
\left(m V_{0}^{2}-T\right) \frac{1}{\ell^{2}} \frac{\partial^{2} w}{\partial x^{2}}+D \frac{1}{\ell^{4}} \frac{\partial^{4} w}{\partial x^{4}}=q_{\mathrm{f}},
$$

where $x \in[-1,1]$. The primes have been omitted from the notation. Transferring $q_{\mathrm{f}}$ to the left-hand side, inserting the final solution for the aerodynamic reaction from (6.41), and dropping the time dependence in it, we obtain

$$
\begin{gathered}
\left(m V_{0}^{2}-T\right) \frac{1}{\ell^{2}} \frac{\partial^{2} w}{\partial x^{2}}+D \frac{1}{\ell^{4}} \frac{\partial^{4} w}{\partial x^{4}} \\
+\rho_{\mathrm{f}} \frac{1}{\ell} v_{\infty} \frac{\partial w}{\partial x} \int_{-1}^{1} N(\xi, x) v_{\infty} \frac{\partial w}{\partial x} w(\xi) \mathrm{d} \xi=0 .
\end{gathered}
$$

Multiplying (6.69) by the factor

$$
\frac{\ell^{2}}{m C^{2}}
$$

and applying (6.62-6.65), we obtain

$$
\left(c^{2}-1\right) \frac{\partial^{2} w}{\partial x^{2}}+\beta \frac{\partial^{4} w}{\partial x^{4}}+\gamma \theta^{2} \frac{\partial}{\partial x}\left[\int_{-1}^{1} N(\xi, x)\left(\frac{\partial}{\partial x} w(\xi)\right) \mathrm{d} \xi\right]=0 .
$$

This is the fourth-order integro-differential equation that will be solved for investigating the static stability. For the boundary conditions, we take the simply supported conditions (2.7-2.8) (p. 25).

We fix all parameters but one, and treat the remaining parameter as an eigenvalue. In this study, we consider only the axial velocities $V_{0}$ (dimensionless form: $c$ ) and $v_{\infty}$ (dimensionless form: $\theta$ ) as eigenvalues.

The static stability problem asks to determine the critical values of the chosen parameter, such that (6.69) admits nontrivial solutions $w(x) \not \equiv 0$, and the corresponding nontrivial solutions.

In the dynamical behaviour problem, we investigate the full dynamical equation (6.1) 


$$
m \frac{\partial^{2} w}{\partial t^{2}}+2 m V_{0} \frac{\partial^{2} w}{\partial x \partial t}+\left(m V_{0}^{2}-T\right) \frac{\partial^{2} w}{\partial x^{2}}+D \frac{\partial^{4} w}{\partial x^{4}}=q_{\mathrm{f}}+g,
$$

describing small transverse vibrations of a travelling panel submerged in potential flow. Refer to Fig. 6.2 in Sect. 6.1 for the setup. The external load $g \equiv g(x, t)$ is allowed to be nonzero, and is considered given.

Let us move into dimensionless coordinates. Let $x^{\prime}=x / \ell$ and $t^{\prime}=t / \tau$, and for the displacement $w$, choose the scaling $w^{\prime}=w / \ell$ that is especially convenient for this problem. We have

$$
\begin{gathered}
m \frac{1}{\tau^{2}} \frac{\partial^{2} w}{\partial t^{2}}+2 m V_{0} \frac{1}{\ell \tau} \frac{\partial^{2} w}{\partial x \partial t} \\
+\left(m V_{0}^{2}-T\right) \frac{1}{\ell^{2}} \frac{\partial^{2} w}{\partial x^{2}}+D \frac{1}{\ell^{4}} \frac{\partial^{4} w}{\partial x^{4}}=\frac{1}{\ell} q_{\mathrm{f}}+\frac{1}{\ell} g .
\end{gathered}
$$

The $1 / \ell$ factor on the right-hand side comes from the scaling of $w=\ell w^{\prime}$ after division of both sides by $\ell$. The dimensionless variables have the ranges

$$
x \in[-1,1] \text { and } t \in[0, \infty) .
$$

The primes have been dropped from the notation.

Next, let us insert the aerodynamic reaction

$$
q_{\mathrm{f}} \equiv q_{\mathrm{f}}(w),
$$

which is given in dimensionless coordinates in (6.41)

$$
q_{\mathrm{f}}=-\rho_{\mathrm{f}} \frac{1}{\ell}\left(\frac{\ell}{\tau} \frac{\partial}{\partial t}+v_{\infty} \frac{\partial}{\partial x}\right) \int_{-1}^{1} N(\xi, x)\left(\frac{\ell}{\tau} \frac{\partial}{\partial t}+v_{\infty} \frac{\partial}{\partial x}\right) w(\xi, t) \mathrm{d} \xi .
$$

In the formula for the aerodynamic reaction, the scaling of $w$ has not been applied yet; only dimensionless $x^{\prime}$ and $t^{\prime}$ are used. Let us apply it now, to get an equation which depends only on the scaled $w^{\prime}$. Inserting $w=\ell w^{\prime}$ into (6.41) eliminates the leading $1 / \ell$. However, when we insert the resulting expression into (6.71), the same factor will be brought back by the $1 / \ell$ multiplying the $q_{\mathrm{f}}$.

Summarizing, we obtain

$$
\begin{gathered}
m \frac{1}{\tau^{2}} \frac{\partial^{2} w}{\partial t^{2}}+2 m V_{0} \frac{1}{\ell \tau} \frac{\partial^{2} w}{\partial x \partial t}+\left(m V_{0}^{2}-T\right) \frac{1}{\ell^{2}} \frac{\partial^{2} w}{\partial x^{2}}+D \frac{1}{\ell^{4}} \frac{\partial^{4} w}{\partial x^{4}}= \\
-\rho_{\mathrm{f}} \frac{1}{\ell}\left(\frac{\ell}{\tau} \frac{\partial}{\partial t}+v_{\infty} \frac{\partial}{\partial x}\right) \int_{-1}^{1} N(\xi, x)\left(\frac{\ell}{\tau} \frac{\partial}{\partial t}+v_{\infty} \frac{\partial}{\partial x}\right) w(\xi, t) \mathrm{d} \xi+\frac{1}{\ell} g(x, t) .
\end{gathered}
$$


Anticipating upcoming manipulations, let us define the dimensionless external load

$$
g^{\prime}\left(x^{\prime}, t^{\prime}\right) \equiv \frac{\ell}{m C^{2}} g\left(\ell x^{\prime}, \tau t^{\prime}\right)=\frac{\ell}{T} g\left(\ell x^{\prime}, \tau t^{\prime}\right) .
$$

where $g$ is the original (dimensional) load function.

We multiply (6.72) by the factor $\ell^{2} / m C^{2}$, and in the $q_{\mathrm{f}}$ term, distribute the $1 / C^{2}$ into the operators (one $1 / C$ per instance of the operator). Applying (6.62-6.66) to the result, and moving $q_{\mathrm{f}}$ to the left-hand side, we have

$$
\begin{aligned}
& \alpha^{2} \frac{\partial^{2} w}{\partial t^{2}}+2 \alpha c \frac{\partial^{2} w}{\partial x \partial t}+\left(c^{2}-1\right) \frac{\partial^{2} w}{\partial x^{2}}+\beta \frac{\partial^{4} w}{\partial x^{4}} \\
& +\gamma\left(\alpha \frac{\partial}{\partial t}+\theta \frac{\partial}{\partial x}\right) \int_{-1}^{1} N(\xi, x)\left(\alpha \frac{\partial}{\partial t}+\theta \frac{\partial}{\partial x}\right) w(\xi, t) \mathrm{d} \xi=g(x, t),
\end{aligned}
$$

where all the variables and functions are in the dimensionless form. The domain of $(6.74)$ is

$$
(x, t) \in((-1,1),(0, \infty)) .
$$

This is the equation that will be solved to determine the dynamical behaviour.

As the boundary conditions, we use the simply supported conditions (2.72.8). Uniqueness of dynamic behaviour requires two initial conditions, as the equation is of the second order in time. We set

$$
\begin{gathered}
w(x, 0)=g_{1}(x) \\
\frac{\partial w}{\partial t}(x, 0)=g_{2}(x),
\end{gathered}
$$

where $g_{1}(x)$ and $g_{2}(x)$ are given functions.

The dynamical problem is to solve the initial boundary value problem (6.74), $(2.7-2.8)$ to find the time-dependent behaviour $w(x, t)$.

The last of the three problems, the corresponding eigenfrequency problem, is concerned with finding the unloaded time-harmonic behaviour. For this, we start from (6.74), and set the load to zero, $g \equiv 0$. By inserting the timeharmonic trial function

$$
w(x, t) \equiv e^{s t} W(x)
$$

into (6.74), and ignoring the common exp factor, we have the pseudo-steadystate problem 


$$
\begin{aligned}
\alpha^{2} s^{2} W+ & 2 \alpha c s \frac{\partial W}{\partial x}+\left(c^{2}-1\right) \frac{\partial^{2} W}{\partial x^{2}}+\beta \frac{\partial^{4} W}{\partial x^{4}} \\
& +\gamma\left(\alpha s+\theta \frac{\partial}{\partial x}\right) \int_{-1}^{1} N(\xi, x)\left(\alpha s+\theta \frac{\partial}{\partial x}\right) W(\xi) \mathrm{d} \xi=0 .
\end{aligned}
$$

Because our original operator is linear in $w$, and the original coefficients are real, the complex-valued time-harmonic approach is justified: the real and imaginary parts of the complex-valued solution will be real-valued solutions of the original problem. However, as usual, $s$ will be complex, and the coefficients of the space-component problem (6.78) will be complex-valued.

As the boundary conditions for $W(x)$, also in this case we choose the simply supported conditions. In the eigenfrequency analysis, we only look for possible modes for free vibrations, so initial conditions are not needed. We have an eigenvalue problem for pairs $(s, W)$, to determine the values for the complex stability exponent, and the corresponding vibration modes.

\subsubsection{Numerical considerations}

For the purposes of numerical analysis, a discrete approximation will be used for the partial differential equations as usual. In the traditional manner, the space discretization will be performed first, producing a semi-discrete form. Deriving the semi-discrete form is the purpose of the present section.

In the dynamical behaviour problem, we will also need a time discretization. This will be performed during the analysis of the dynamic problem. For the other two problems, the space discretization is sufficient. The steadystate problem is by definition time-independent, and for the eigenfrequency problem, the time component is known analytically.

Galerkin methods are especially convenient for numerical handling of integro-differential equations. We will space-discretize using the FourierGalerkin method (see e.g. Canuto et al 1988). The Fourier-Galerkin method is a traditional spectral method of the Galerkin type. According to Canuto et al (1988), the first serious application of spectral methods to partial differential equations was made by Silberman (1954) for meteorological modelling. A stability analysis of the method and some further references are available in Tadmor (1987). Recently, the method has been applied, e.g., for computing the stationary solutions of two-dimensional generalised wave equations by Christou and Christov (2007).

We represent the displacement $w$ as a Galerkin series,

$$
w(x, t)=\sum_{n=1}^{\infty} f_{n}(t) \Psi_{n}(x)
$$


We work in dimensionless coordinates, so there is no scaling factor, and $w$, which is actually $w^{\prime}$ with the prime omitted from notation, is dimensionless. The functions $f_{n}$ and $\Psi_{n}$ are both dimensionless.

For the shape functions $\Psi_{n}$, we choose the eigenmodes of free vibrations of a stationary string in vacuum,

$$
\Psi_{n}(x) \equiv \sin \left(n \pi \frac{x+1}{2}\right), \quad x \in[-1,1] .
$$

This is a Fourier sine basis that splits the space component of the solution into a frequency domain along the $x$ axis. This use of the word frequency here has nothing to do with time, but represents the decomposition of the space axis in a manner somewhat similar to what Fourier analysis does to time signals. The functions $f_{n}$ are functions of the time $t$ directly, hence our frequency domain is only spatial.

By construction, the chosen basis automatically accounts for the simply supported boundary conditions $(2.7-2.8)$. The basis is equivalent to the standard Fourier sine basis, up to a constant factor of 2 , if the solution is considered to have period 4 , and to be antisymmetric with regard to the (fictitious) midpoint $x=1$.

We start the development of the semi-discrete form from the dimensionless integro-differential equation (6.74), which we will repeat here:

$$
\begin{aligned}
& \alpha^{2} \frac{\partial^{2} w}{\partial t^{2}}+2 \alpha c \frac{\partial^{2} w}{\partial x \partial t}+\left(c^{2}-1\right) \frac{\partial^{2} w}{\partial x^{2}}+\beta \frac{\partial^{4} w}{\partial x^{4}} \\
& +\gamma\left(\alpha \frac{\partial}{\partial t}+\theta \frac{\partial}{\partial x}\right) \int_{-1}^{1} N(\xi, x)\left(\alpha \frac{\partial}{\partial t}+\theta \frac{\partial}{\partial x}\right) w(\xi, t) \mathrm{d} \xi=g(x, t) .
\end{aligned}
$$

One must be careful with the $\partial / \partial x$ in front of the integral in the fluid term, because the aerodynamic kernel $N(\xi, x)$ is singular. It is not possible to directly take the derivative operator into the integral, because the $L_{1}$ norm of $\partial N / \partial x$ is not finite. A straightforward, but somewhat lengthy, calculation finds that $\partial N / \partial x$ has singularities of type $1 / x^{a}$, where $a \geq 1$. The singularities are located at $x= \pm 1$ with $a=3 / 2$, and at $x=\xi$ with $a=1$.

However, as was shown in the first section of this chapter, the integral in (6.40) is absolutely convergent, and thus the function $\varphi^{+}$is bounded. It is possible to choose from two approaches. The first approach is to integrate first, and then differentiate the result by any method. The second approach, more applicable here because we will work in the weak form and do not have a closed-form antiderivative, is to integrate by parts against the test function as usual. This is legitimate despite the singularity, because the integrand of the weak form is a product of two bounded, integrable functions.

We now consider the system of equations corresponding to the dynamics of the panel, expressed by the weak form of (6.74). As was mentioned earlier, 
(6.74) is of the abstract form

$$
\mathcal{L}\left(w ; V_{0}\right)+\gamma \mathcal{K}\left(w ; v_{\infty}\right)=\mathcal{G} .
$$

We multiply (6.74) by the test function $\Psi_{j}(x)$ and integrate over the dimensionless space domain $\Omega \equiv\{x \in \mathbb{R} \mid-1<x<1\}$. We have

$$
\begin{gathered}
\alpha^{2} \int_{-1}^{1} \frac{\partial^{2} w}{\partial t^{2}} \Psi_{j} \mathrm{~d} x+2 \alpha c \int_{-1}^{1} \frac{\partial^{2} w}{\partial x \partial t} \Psi_{j} \mathrm{~d} x+ \\
\left(c^{2}-1\right) \int_{-1}^{1} \frac{\partial^{2} w}{\partial x^{2}} \Psi_{j} \mathrm{~d} x+\beta \int_{-1}^{1} \frac{\partial^{4} w}{\partial x^{4}} \Psi_{j} \mathrm{~d} x \\
+\gamma \int_{-1}^{1}\left[\left(\alpha \frac{\partial}{\partial t}+\theta \frac{\partial}{\partial x}\right) \int_{-1}^{1} N(\xi, x)\left(\alpha \frac{\partial}{\partial t}+\theta \frac{\partial}{\partial x}\right) w(\xi, t) \mathrm{d} \xi\right] \Psi_{j}(x) \mathrm{d} x \\
=\int_{-1}^{1} g(x, t) \Psi_{j} \mathrm{~d} x . \quad(6.82)
\end{gathered}
$$

The brackets emphasize that the aerodynamic reaction is one function. In the same term, the $x$ in $\Psi_{j}(x)$ is indicated explicitly, because there are two integrations: first, over the dummy variable $\xi$ to determine $q_{\mathrm{f}}(x, t)$ at a fixed $x$, and then over $x$ from the weak form. From (6.82), the choices of functions for integration by parts should be more apparent, and it should be clear what to do in practice in order to transfer the outer $\partial / \partial x$ to the factor $\Psi_{j}(x)$.

That takes care of the space derivative. What about the outer $\partial / \partial t$ ? Once we insert the Galerkin series (6.79), for each term $n$ in the sum, the function $w$ will separate into a product of space- and time-dependent parts. We make the standard assumption that the series is "convergent enough" so that the order of spatial integration and series summation can be exchanged. Once the summation has been taken outside both (nested) integrals, we can use the fact that the time part is constant in space, and hence it too can be taken outside the integrals.

To sum up, as long as care is taken with the outer $\partial / \partial x$ in the aerodynamic term, the rest can be done in the standard manner. The result is as follows. Inserting the Galerkin series (6.79) into the weak form (6.82) gives us the following expressions for the weak equivalents of the operators $\mathcal{L}$ and $\mathcal{K}$ : 


$$
\begin{gathered}
\int_{-1}^{1} \mathcal{L}(w ; c) \Psi_{j} \mathrm{~d} x=\int_{-1}^{1} \mathcal{L}\left(\sum_{n=1}^{\infty} f_{n} \Psi_{n} ; c\right) \Psi_{j} \mathrm{~d} x= \\
\sum_{n=1}^{\infty}\left[\alpha^{2} A_{j n} \frac{\mathrm{d}^{2} f_{n}}{\mathrm{~d} t^{2}}+2 \alpha c B_{j n} \frac{\mathrm{d} f_{n}}{\mathrm{~d} t}+\left(c^{2}-1\right) C_{j n} f_{n}+\beta D_{j n} f_{n}\right] \\
\int_{-1}^{1} \mathcal{K}(w ; \theta) \Psi_{j} \mathrm{~d} x=\int_{-1}^{1} \mathcal{K}\left(\sum_{n=1}^{\infty} f_{n} \Psi_{n} ; \theta\right) \Psi_{j} \mathrm{~d} x= \\
\sum_{n=1}^{\infty}\left[\alpha^{2} a_{j n} \frac{\mathrm{d}^{2} f_{n}}{\mathrm{~d} t^{2}}+2 \alpha \theta b_{j n} \frac{\mathrm{d} f_{n}}{\mathrm{~d} t}+\theta^{2} c_{j n} f_{n}\right]
\end{gathered}
$$

The operator $\mathcal{L}$ depends on the dimensionless panel velocity $c$, while $\mathcal{K}$ depends on the dimensionless fluid velocity $\theta$. Both operators are nonzero even at zero velocity.

The formal matrices $A_{j n}, B_{j n}, C_{j n}, D_{j n}, a_{j n}, b_{j n}$ and $c_{j n}$ are defined by

$$
\begin{aligned}
A_{j n} & =\int_{-1}^{1} \Psi_{n}(x) \Psi_{j}(x) \mathrm{d} x=\delta_{j n} \\
B_{j n} & =\int_{-1}^{1} \frac{\mathrm{d} \Psi_{n}}{\mathrm{~d} x}(x) \Psi_{j}(x) \mathrm{d} x=\left\{\begin{array}{l}
0, \quad j=n \\
\frac{n j}{n^{2}-j^{2}}\left((-1)^{j+n}-1\right), \quad j \neq n
\end{array}\right. \\
C_{j n} & =\int_{-1}^{1} \frac{\mathrm{d}^{2} \Psi_{n}}{\mathrm{~d} x^{2}}(x) \Psi_{j}(x) \mathrm{d} x=-\left(\frac{j \pi}{2}\right)^{2} \delta_{j n} \\
D_{j n} & =\int_{-1}^{1} \frac{\mathrm{d}^{4} \Psi_{n}}{\mathrm{~d} x^{4}}(x) \Psi_{j}(x) \mathrm{d} x=\left(\frac{j \pi}{2}\right)^{4} \delta_{j n} \\
a_{j n} & =\int_{-1}^{1} \int_{-1}^{1} \Psi_{n}(\xi) N(\xi, x) \Psi_{j}(x) \mathrm{d} \xi \mathrm{d} x \\
b_{j n} & =\frac{1}{2}\left(I_{j n}-I_{n j}\right) \quad \text { and } \\
c_{j n} & =-\int_{-1}^{1} \int_{-1}^{1} \frac{\mathrm{d} \Psi_{n}}{\mathrm{~d} x}(\xi) N(\xi, x) \frac{\mathrm{d} \Psi_{j}}{\mathrm{~d} x}(x) \mathrm{d} \xi \mathrm{d} x
\end{aligned}
$$

where $j, n=1,2,3, \ldots$ and $\delta_{j n}$ is the Kronecker delta. In (6.90), we have defined

$$
I_{j n} \equiv \int_{-1}^{1} \int_{-1}^{1} \frac{\mathrm{d} \Psi_{n}}{\mathrm{~d} x}(\xi) N(\xi, x) \Psi_{j}(x) \mathrm{d} \xi \mathrm{d} x .
$$

The test functions are indexed by $j$; the Galerkin series is summed over $n$.

In (6.91), and in the $I_{n j}$ term in (6.90), we have carried out the mentioned integration by parts. No boundary terms appear, because $N(\xi, \pm 1)=0$ either directly, at $x=+1$, or in the sense of limits, at $x=-1$. This holds for any 
choice of boundary conditions for $w$. Additionally, if the boundary conditions require

$$
w=0, \quad \text { at } x= \pm 0,
$$

then it follows that

$$
\Psi_{j}( \pm 1)=0 \quad \text { for all } j .
$$

Although we use a global sine basis which is infinitely smooth, the integration by parts is compulsory due to the singularity of $N(\xi, x)$.

The closed-form solutions of (6.85-6.88) are, obviously, specific to the basis (6.80). In all other respects, the definitions (6.85-6.92) always hold, regardless of the basis or boundary conditions chosen.

The integrals in (6.89-6.92) have no closed-form solution, but some useful properties may be obtained analytically, assuming the basis (6.80). A summary follows; for details, see Jeronen (2011). If $j+n$ is odd, then

$$
a_{j n}=c_{j n}=0
$$

by considering the symmetries of each integrand. The matrix $b_{j n}$ is antisymmetric, and if $j+n$ is even, then $b_{j n}=0$. The matrices $a_{j n}$ and $c_{j n}$ are symmetric by the symmetry of $N(\xi, x)$ with respect to the line $x=\xi$ and the application of Fubini's theorem. When $j+n$ is even, each integrand $a_{j n}$ and $c_{j n}$ is symmetric with respect to the lines $x=\xi$ and $x=-\xi$.

Summarizing, starting from (6.74), we have obtained the semidiscrete weak form,

$$
\begin{aligned}
\sum_{n=1}^{\infty}\left(\alpha ^ { 2 } \left[A_{j n}+\right.\right. & \left.\gamma a_{j n}\right] \frac{\mathrm{d}^{2} f_{n}}{\mathrm{~d} t^{2}}+2 \alpha\left[c B_{j n}+\gamma \theta b_{j n}\right] \frac{\mathrm{d} f_{n}}{\mathrm{~d} t} \\
& \left.+\left[\left(c^{2}-1\right) C_{j n}+\gamma \theta^{2} c_{j n}+\beta D_{j n}\right] f_{n}\right)-G_{j}=0,
\end{aligned}
$$

where definitions (6.62-6.66) and (6.85-6.92) have been used. Additionally, we have defined the space-discrete load vector, whose $j$ th component is

$$
G_{j}(t) \equiv \int_{-1}^{1} g(x, t) \Psi_{j}(x) \mathrm{d} x,
$$

where all the functions and variables are dimensionless.

Finally, consider the initial conditions for direct temporal simulation. We insert (6.79) into $(6.75-6.76)$ (page 158), multiply both sides by $\Psi_{j}(x)$, integrate over the domain, and use (6.85) to eliminate all cross terms on the left-hand side. We have

$$
f_{j}(0)=\frac{1}{\ell} \int_{-1}^{1} \Psi_{j}(x) g_{1}(x) \mathrm{d} x, \quad j=1,2,3, \ldots
$$




$$
\frac{\mathrm{d} f_{j}}{\mathrm{~d} t}(0)=\frac{1}{\ell} \int_{-1}^{1} \Psi_{j}(x) g_{2}(x) \mathrm{d} x, \quad j=1,2,3, \ldots .
$$

The leading $1 / \ell$ comes from the scaling $w=\ell w^{\prime}$, by dividing both sides of each equation by $\ell$.

This completes the semi-discrete form. The original initial boundary value problem has been transformed into a Cauchy problem for the system of ordinary differential equations (6.93) with initial conditions (6.95-6.96).

For a practical numerical implementation, we truncate the Galerkin series at a given value of $n=n_{0}$. The resulting finite equation corresponding to (6.93) is a linear, second-order, non-homogeneous, system of ordinary differential equations with constant coefficients.

We will consider the three main problems one at a time. We will discuss the steady-state problem, direct temporal integration (dynamical behaviour problem), and the eigenfrequency problem. The treatment of the last one is split into two parts. We will first look at the lowest eigenfrequency, and then at computing as many eigenfrequencies as the discretization allows. For each problem, we will first explain the solution strategy, starting from the semi-discrete form that was derived above. We will then consider numerical examples. The handling of each problem ends with a short summary and conclusion.

Problem parameters used in all numerical examples are given in Table 6.1. The physical parameters have been chosen as typical for a paper web surrounded by air. For the bending rigidity $D$, zero is used for some calculations; this is mentioned when it is the case. Otherwise, the value in Table 6.1 is used.

\subsubsection{Static stability analysis}

As was mentioned above, let us first outline the numerical solution strategy. The discrete steady-state problem, for numerical static stability analysis, can be obtained directly from the semidiscrete form, (6.93), by setting the time scale parameter to $\alpha=0$ (i.e., $\tau \rightarrow \infty$ ) and assuming zero external load, $G_{j} \equiv 0$. We have

$$
\sum_{n=1}^{\infty}\left[\left(c^{2}-1\right) C_{j n}+\gamma \theta^{2} c_{j n}+\beta D_{j n}\right] f_{n}=0 .
$$

Compare the continuum formulation in (6.70), p. 156. Note that (6.97) is in

weak form, while the original equation (6.70) is in strong form. The finite equation corresponding to (6.97) is an algebraic, linear equation system. The discrete problem is to find the critical values of the chosen parameter, such 
Table 6.1 Problem parameters used. Physical parameters are the fluid density $\rho_{\mathrm{f}}$, axial tension $T$, mass per unit area of the panel $m$, span half-length $\ell$ (refer to Fig. 6.2 in Sect. 6.1), characteristic time $\tau$, panel thickness $h$, Young modulus $E$, Poisson ratio $\nu$, and bending rigidity $D$. Dimensionless parameters $\alpha, \beta, \gamma, \theta$ and $c$ are as defined in (6.62-6.66).

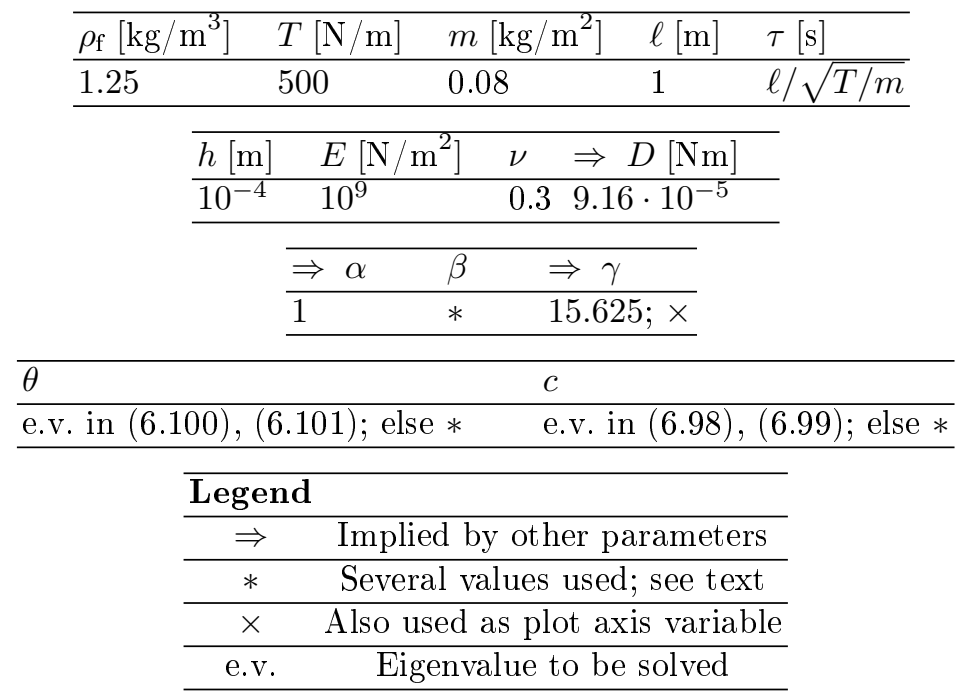

that (6.97) admits nontrivial steady-state solutions $\mathbf{f} \not \equiv 0$, and to determine those nontrivial solutions.

Let us truncate the series (6.97) at some $n_{0}$. First, consider the panel velocity $V_{0}$ (respectively $c$ in dimensionless form) as the eigenvalue. If $v_{\infty}$ is independent of $V_{0}$, we can rearrange the terms of (6.97) to produce the following generalized linear eigenvalue problem in $\left(c^{2}, \mathbf{f}\right)$ :

$$
\sum_{n=1}^{n_{0}}\left[C_{j n}-\gamma \theta^{2} c_{j n}-\beta D_{j n}\right] f_{n}=c^{2} \sum_{n=1}^{n_{0}} C_{j n} f_{n} .
$$

If $v_{\infty}=V_{0}$ (resp. $\theta=c$; the whole air mass moves with the panel), the problem for $\left(c^{2}, \mathbf{f}\right)$ reads

$$
\sum_{n=1}^{n_{0}}\left[C_{j n}-\beta D_{j n}\right] f_{n}=c^{2} \sum_{n=1}^{n_{0}}\left(C_{j n}+\gamma c_{j n}\right) f_{n} .
$$

From this discrete form, it is apparent that the model described by (6.99) is similar in spirit to the corresponding vacuum model, as there is only one independent velocity. Indeed, the form of the equation is a perturbed vacuum model with the additional term $\gamma c_{j n}$. In the limit of zero dimensionless fluid density, $\gamma \rightarrow 0$, it reduces to the vacuum model. Compare (6.98), which 
reduces to the vacuum model not only when the fluid density $\gamma \rightarrow 0$, but also when the dimensionless fluid velocity $\theta \rightarrow 0$. This is because in the steady-state problem, the part of the operator $\mathcal{K}$ that does not depend on the velocity $\theta$ is not present; it would be in the term with the second-order time derivative. In the dynamic case, where this part is present, the model differs from the vacuum model even for $\theta=0$. Hence, we can expect different behaviour from the two cases.

Now consider the fluid velocity $v_{\infty}$ (respectively $\theta$ in dimensionless form) as the eigenvalue. The problem for $\left(\theta^{2}, \mathbf{f}\right.$ ) (now for fixed $V_{0}$, i.e., $c$ ) reads

$$
\sum_{n=1}^{n_{0}}\left[\left(1-c^{2}\right) C_{j n}-\beta D_{j n}\right] f_{n}=\theta^{2} \sum_{n=1}^{n_{0}} \gamma c_{j n} f_{n} .
$$

The special case $c=0$ of (6.100) corresponds to a stationary, tensioned panel subjected to axial flow; see e.g. Kornecki et al (1976) and Païdoussis (2004, 2008). This case has been experimentally tested in a wind tunnel, for a stationary web held fixed at two edges and free to move on two others, by Chang and Moretti (1991). In the article, the authors considered a threadline model for cylindrical displacement, with added masses to account for the surrounding flow. We obtain the corresponding problem in the limit of an infinitely wide wind tunnel, i.e., assuming the walls are far enough not to disturb the flow near the panel, by setting $\beta=0$ and $c=0$ in (6.100). We have

$$
\sum_{n=1}^{n_{0}} C_{j n} f_{n}=\theta^{2} \sum_{n=1}^{n_{0}} \gamma c_{j n} f_{n}
$$

which is the eigenvalue problem for $\left(\theta^{2}, \mathbf{f}\right)$ corresponding to Chang and Moretti (1991).

The classical non-tensioned panel requires different treatment, because we have divided by the tension $T$ to produce the dimensionless form. Guo and Païdoussis (2000) (see also Païdoussis 2004) have considered the stability of a classical non-tensioned plate subjected to two-dimensional axial flow in a channel. In our notation, the continuum formulation reads

$$
D \frac{\partial^{4} w}{\partial x^{4}}=\ell^{3} q_{\mathrm{f}} .
$$

Let us rewrite (6.97) without the dimensionless forms (6.62-6.65):

$$
\sum_{n=1}^{\infty}\left[\left(\frac{m}{\ell} V_{0}^{2}-\frac{T}{\ell}\right) C_{j n}+\frac{D}{\ell^{3}} D_{j n}+\rho_{\mathrm{f}} v_{\infty}^{2} c_{j n}\right] f_{n}=0 .
$$

By setting $T=0$ and $V_{0}=0$ from (6.102), we can formulate this problem, again in the limit of an infinitely wide channel, for $\left(\bar{U}^{2}, \mathbf{f}\right)$ : 


$$
-\sum_{n=1}^{\infty} D_{j n} f_{n}=\bar{U}^{2} \sum_{n=1}^{\infty} c_{j n} f_{n}
$$

where the eigenvalue $\bar{U}^{2}$ is the dimensionless dynamic pressure (Païdoussis 2004)

$$
\bar{U}^{2}=\frac{\rho_{\mathrm{f}} \ell^{3}}{D} v_{\infty}^{2}
$$

which is the square of the dimensionless flow velocity $\bar{U}$. The critical fluid flow velocity for divergence, $v_{\infty}^{\text {div }}$, can be found from ${\overline{U_{\mathrm{cd}}}}^{2}$, the minimal positive eigenvalue $\bar{U}^{2}$ of the problem (6.103).

All the problems (6.98-6.101), (6.103) are generalized linear eigenvalue problems; i.e. are of the general form

$$
\mathbf{A}_{1} \mathbf{Y}=\varkappa \mathbf{A}_{2} \mathbf{Y}
$$

with $\mathbf{A}_{2}$ a non-identity matrix. Here $\mathbf{A}_{1}$ is an arbitrary matrix, $\varkappa$ is the (scalar) eigenvalue and $\mathbf{Y}$ is the corresponding eigenvector. Standard eigenvalue solvers can be used to solve (6.105). Refer to Golub and van Loan (1996) for algorithmic details if needed.

Next, we will look into some numerical examples for these steady-state problems. A parametric study will be performed for problems (6.98) and (6.99). The critical velocities predicted from (6.99) will be compared to Pramila (1986). Additionally, the results of (6.101) will be compared to those in Chang and Moretti (1991), and the results from (6.103) will be compared to Guo and Païdoussis (2000).

In all cases, the Galerkin series is truncated at $n_{0}=56$. For problems (6.98) and (6.99), physical parameters given in Table 6.1 on page 165 are used.

For problem (6.101), the physical parameters are chosen as corresponding to those of Chang and Moretti (1991). The parameter values are $D=0$, $m=0.076680634 \mathrm{~kg} / \mathrm{m}^{2}, c=0$. The width of the plate, needed in the theory of Chang and Moretti (1991), is $b=0.1524 \mathrm{~m}$. Note that the model described in this book does not use the width. Two cases are illustrated; in case $\mathrm{A}$, plate length is $2 \ell=0.508 \mathrm{~m}$. In case $\mathrm{B}$, length $2 \ell=0.254 \mathrm{~m}$. The compared theoretical results are calculated from Chang and Moretti (1991, eq. (20)), which states

$$
v_{\infty}^{\mathrm{div}}=\sqrt{\frac{T}{m_{\mathrm{a}}}}, \quad \text { where } \quad m_{\mathrm{a}}=\frac{\pi}{4} \rho_{\mathrm{f}} b .
$$

The symbol $T$ represents the tension per unit width of web, and $b$ is the web width. Because in this problem the web itself is stationary, only the added mass $m_{\mathrm{a}}$ appears in the expression for $v_{\infty}^{\text {div }}$ and it does not depend on the web mass $m$. 
Now, let us look at the effect of problem parameters on the critical panel velocity c. Figures $6.11-6.14$ show results calculated from problem (6.98), where the dimensionless fluid velocity $\theta$ is a parameter. Figure 6.11 shows an example of the critical shape (buckling mode, divergence mode), and the difference between the vacuum mode and the with-fluid mode for some parameter values.

Consider Fig. 6.12. As expected, the region of stability expands toward materials having a larger bending stiffness $D$, or as the length of the span $\ell$ becomes shorter. Both of these changes increase $\beta$. However, as the tension $T$ is increased, $\beta$ decreases. This is counterintuitive, as one would expect a stabilising effect resulting from increased tension. A stabilising effect is indeed present, because increasing the tension also increases the vacuum divergence velocity, which is used in the normalization of the dimensionless quantities. This causes $\theta$ to decrease if the dimensional fluid velocity is kept constant, and at $\theta=0, c$ stays constant with increasing $T$. Thus, one needs to be careful when interpreting the dimensionless results with respect to tension $(T)$ or panel area mass $(m)$.

Because static instability analysis is only concerned with the critical velocity, and not the complex-valued eigenfrequencies, it cannot tell apart the type of the interval between the origin $\left(V_{0}=0\right)$ and the minimal positive eigenvalue, i.e., whether the behaviour in the interval is stable or unstable. Of course, by physical considerations this interval is known to be stable when the fluid velocity is zero.

We concentrate in this analysis only on the lowest region of stability, given by the interval between the two eigenvalues that, at zero fluid velocity, are the maximal negative and the minimal positive eigenvalue. Once either end of this region crosses the origin, as $\theta$ is varied, the results become physically meaningless. The figures shown have been filtered to show only the physically meaningful data. The critical value of $\theta=\theta_{\text {critical }}$, where the lowest region of stability ends, can be found for each fixed pair $(\beta, \gamma)$ numerically e.g. with a linear-logarithmic search procedure. That is, start from $\theta=0$, increase $\theta$ in fixed steps until $c^{2}<0$, then go back one step, halve the step size and repeat the procedure until desired tolerance is achieved. However, in order for this to work correctly, the starting step size should be small enough. Otherwise the search may find a higher stability region, where again $c^{2}>0$, which occurs in the linear model starting at some value of $\theta$ larger than $\theta_{\text {critical }}$.

Figure 6.13 shows the effects of $\gamma$ and $\theta$ on the critical velocity $c$. The contour plot is symmetric with respect to $\theta$, and hence only the positive half is shown. This feature is to be expected, because (6.98) only depends on $\theta^{2}$. Figure 6.14 shows axis-oriented slices of the contour at various parameter values.

Figures $6.15-6.17$ illustrate the solutions of problem (6.99), where the whole air mass moves with the panel, i.e., $v_{\infty}=V_{0}$, and $\theta=c=$ eigenvalue. Figure 6.15 again shows an example of the critical shape (buckling mode, divergence mode), and its difference from the vacuum solution for some values 

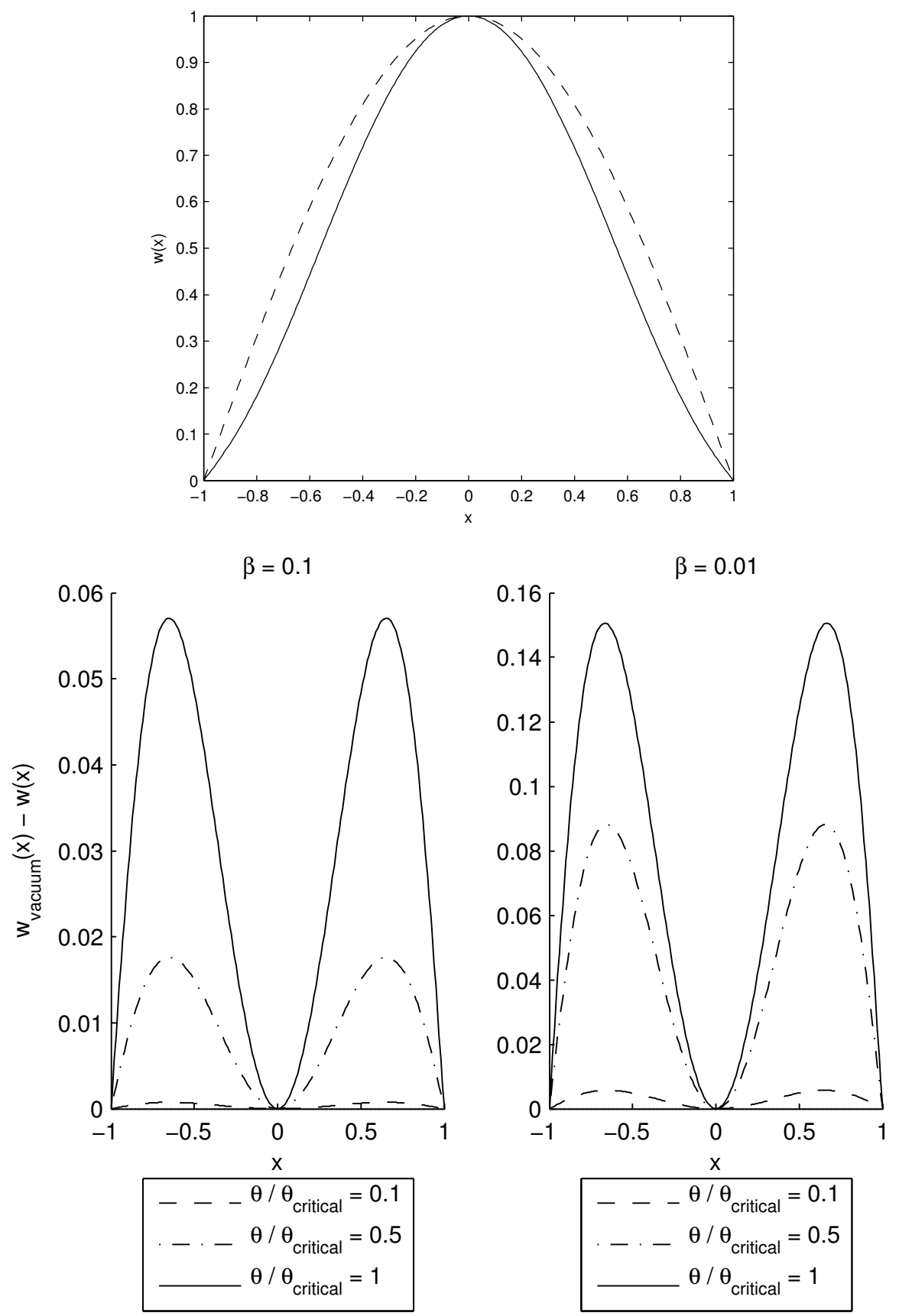

Fig. 6.11 Some solutions of problem (6.98), where the dimensionless fluid velocity $\theta$ is a parameter. The dimensionless fluid density $\gamma=15.625$. Top: Critical eigenmode (solid line). The dashed line shows the corresponding vacuum mode. Both modes are normalized such that the maximum is 1 . Shapes are very similar for any admissible $\theta$. Bottom: the difference between the vacuum and with-fluid solutions at two dimensionless bending rigidities $\beta$, for different fluid velocities $\theta$. Differences are computed using the same normalization for the solutions as on the top. The symbol $\theta_{\text {critical }}$ denotes the smallest value of $\theta$ at which the dimensionless critical panel velocity $c$ becomes zero (see Fig. 6.12). 


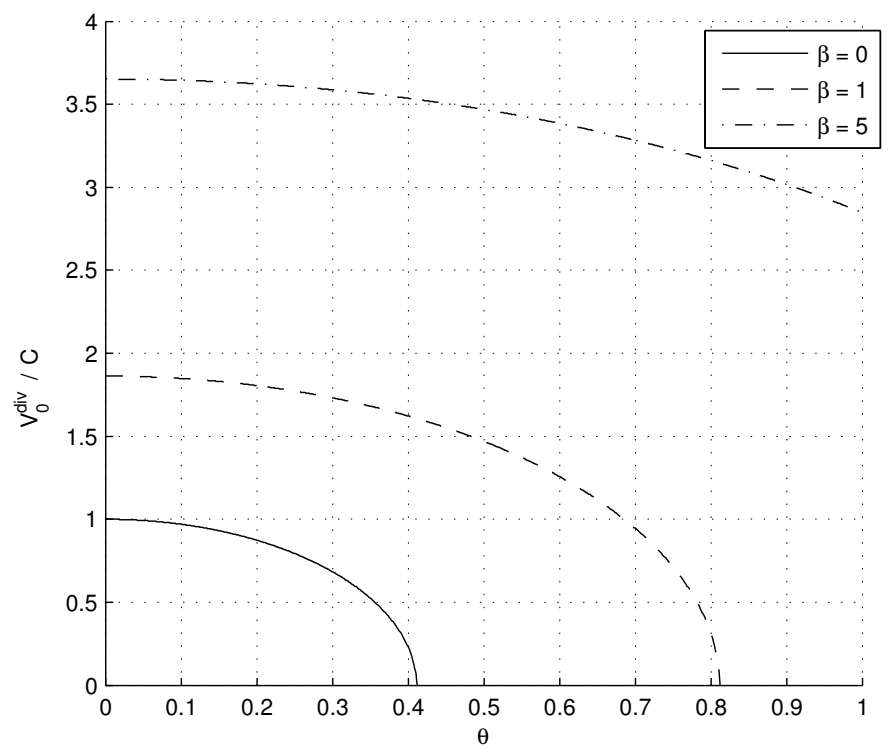

Fig. 6.12 Problem (6.98). Effects of the dimensionless fluid velocity $\theta$ and the dimensionless bending rigidity $\beta$ on the dimensionless critical panel velocity $c$. The dimensionless fluid density $\gamma=15.625$. The result is symmetric for $-\theta$; hence only the positive half is shown. The axis intersection points on the horizontal axis are the values $\theta_{\text {critical }}$ for the corresponding parameter point $(\beta, \gamma)$.

of the dimensionless bending rigidity $\beta$. In Fig. 6.16 we see the effects of $\beta$ and $\gamma$ on the critical velocity $c$. Figure 6.17 again shows axis-oriented slices of the same effect at various parameter values. As expected, an increase in the bending stiffness $\beta$ has a stabilizing influence, while an increase in the fluid density $\gamma$ has a destabilizing influence.

Table 6.2 gives the numerical values of the critical velocity for the reference values of the problem parameters (refer to Table 6.1 on p. 165) at various $v_{\infty}$. The values of $v_{\infty}$ correspond to the choices in Fig. 6.27 in the upcoming lowest eigenfrequency analysis. The vacuum case gives the same critical velocity as $v_{\infty}=0$, so it is not reported separately. This is expected; recall the comments on (6.98). The special case $v_{\infty}=V_{0}$ is also shown. We see that with this assumption, using our parameter values we obtain a critical velocity that is about $39 \%$ of the corresponding vacuum value.

Let us compare these results to those by Pramila (1986). In Pramila (1986), an axially moving web submerged in ideal fluid was considered. We have $v_{\infty}=0$ for the stationary air, $D=0$ for the threadline model (corresponding to an ideal membrane in the panel model), and $\rho_{\mathrm{f}}=1.2 \mathrm{~kg} / \mathrm{m}^{3}$. However, since in our model the fluid does not affect the critical velocity when $v_{\infty}=0$, it is more natural to assume $v_{\infty}=V_{0}$ as an alternative model for the case with no free stream. 


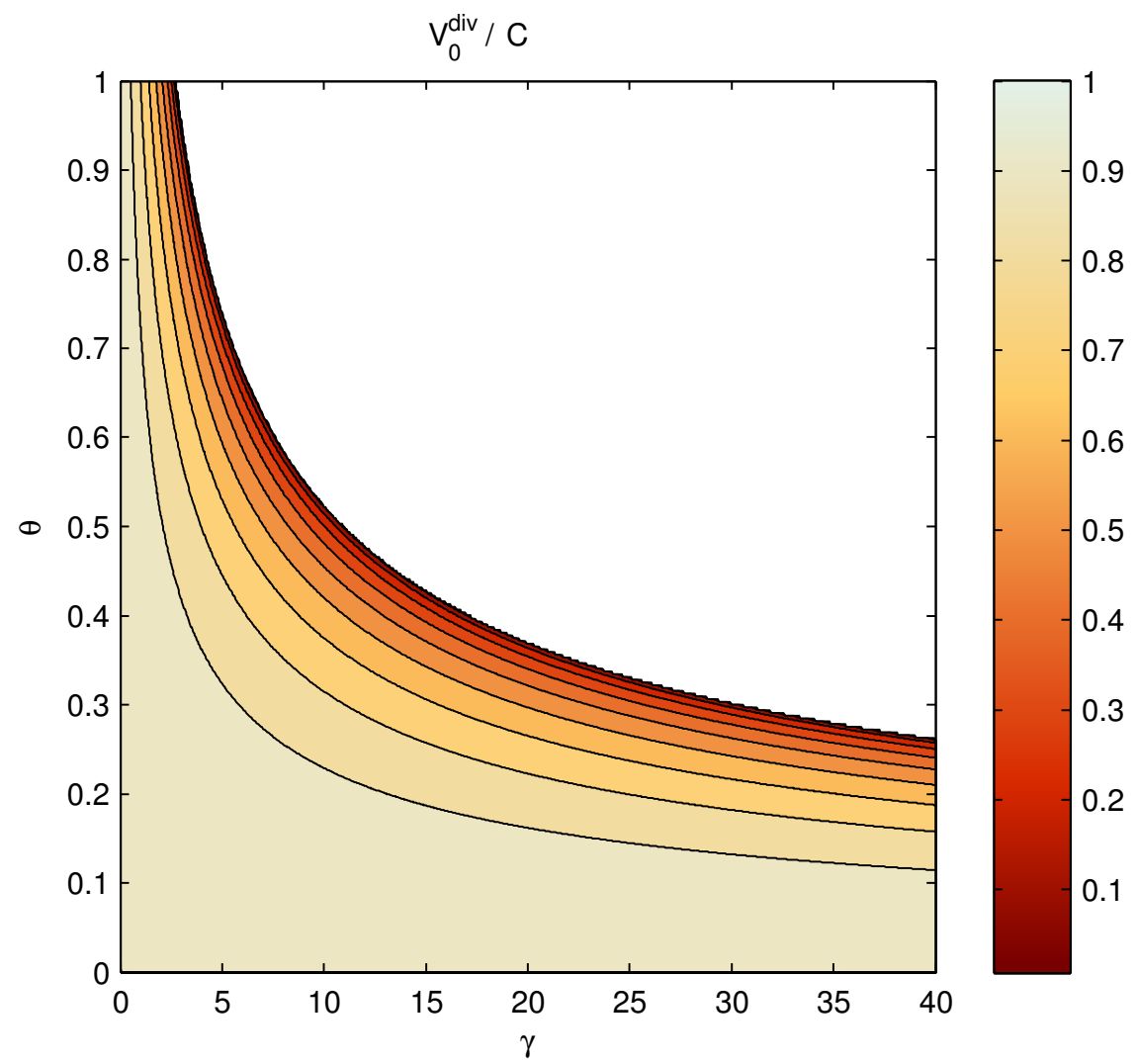

Fig. 6.13 Problem (6.98). Effects of the dimensionless fluid density $\gamma$ and the dimensionless fluid velocity $\theta$ on the dimensionless critical panel velocity $c$. The result is symmetric for $-\theta$; hence only the positive half is shown. The bending rigidity is fixed to $\beta=0$; the solution is representative for small bending rigidities. On the axes, $c \equiv 1$. In the blank area, the problem has no physically meaningful solution. See also slices of this contour plot in Fig. 6.14.

Table 6.2 Critical panel velocity $V_{0}$ as a function of fluid velocity $v_{\infty}$. The solution does not depend on the signs of the velocities. The same data are given in both dimensional and dimensionless forms; given a value of $v_{\infty}$ (respectively $\theta$ ), the critical panel velocity is $V_{0}^{\text {div }}\left(\right.$ respectively $c^{\text {div }}$ ). The dimensionless critical velocities $c^{\text {div }}$ give the axis intersection points of Fig. 6.27 (p. 196).

\begin{tabular}{lllllllll}
\hline$v_{\infty}[\mathrm{m} / \mathrm{s}]$ & 0 & 10 & 15 & 20 & 25 & 30 & $v_{\infty}=V_{0}$ \\
\hline$V_{0}^{\text {div }}[\mathrm{m} / \mathrm{s}]$ & 79.057 & 75.385 & 70.526 & 63.098 & 52.012 & 33.845 & 30.607 \\
\hline \hline$\theta$ & 0 & 0.13 & 0.19 & 0.25 & 0.32 & 0.38 & $\theta=c$ \\
\hline$c^{\text {div }}$ & 1 & 0.95355 & 0.89209 & 0.79813 & 0.65791 & 0.42811 & 0.38715 \\
\hline
\end{tabular}



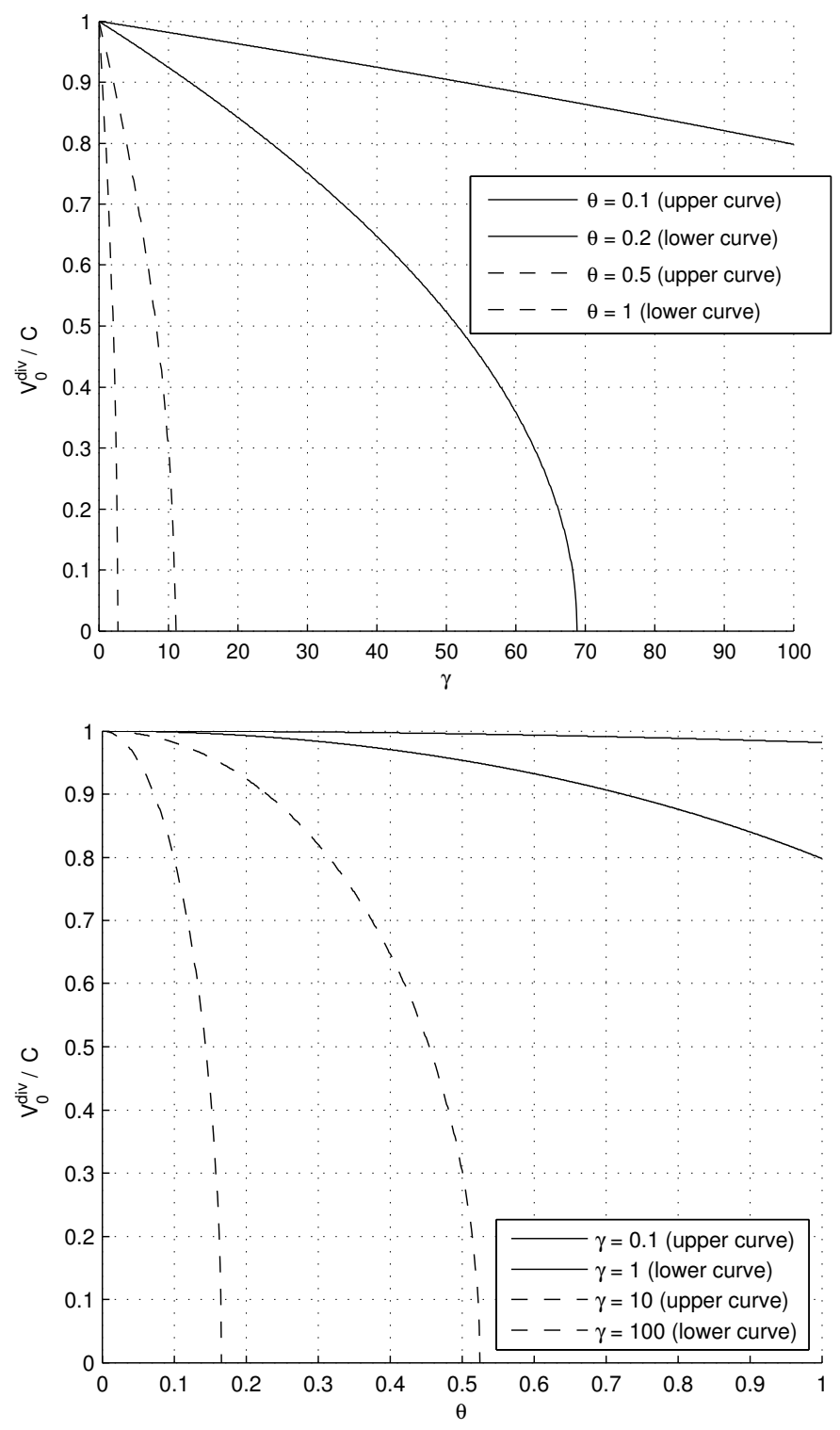

Fig. 6.14 Problem (6.98). Effects of the dimensionless fluid density $\gamma$ and the dimensionless fluid velocity $\theta$ on the dimensionless critical panel velocity $c$. Top: effect of density $\gamma$. For $\theta=0, c \equiv 1$ (not plotted). Bottom: effect of fluid velocity $\theta$. For $\gamma=0, c \equiv 1$ (not plotted). The bending rigidity is fixed to $\beta=0$; the solution is representative for small bending rigidities. See also the contour plot in Fig. 6.13. 

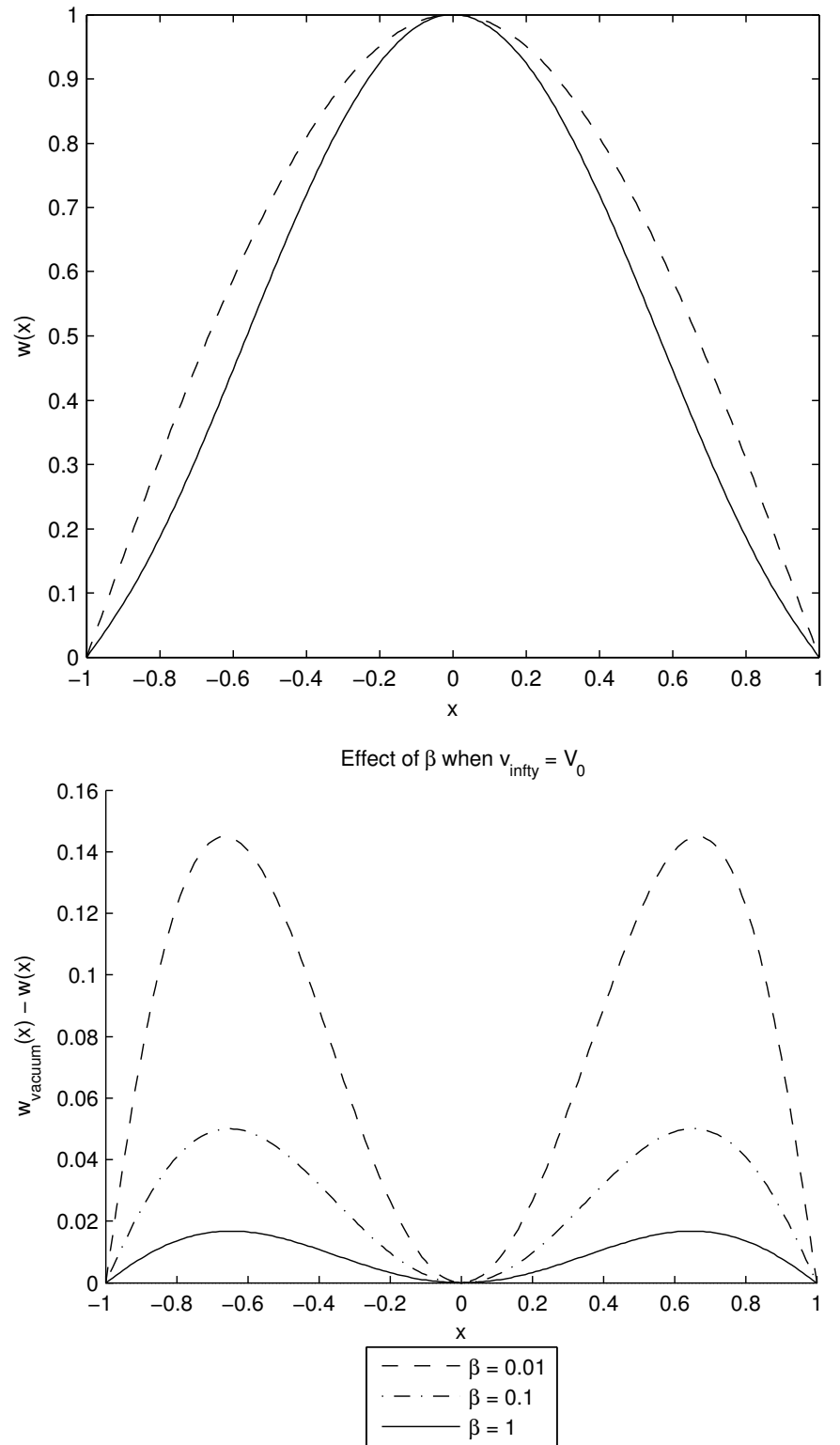

Fig. 6.15 Some solutions of problem (6.99), where the air mass moves with the panel. The dimensionless density $\gamma=15.625$. Top: Critical eigenmode (solid line). The dashed line shows the corresponding vacuum mode. Both modes normalized such that the maximum is 1. Bottom: the difference between the vacuum and with-fluid solutions at different values for the dimensionless bending rigidity $\beta$. Differences computed using the same normalization for the solutions as on the left. 


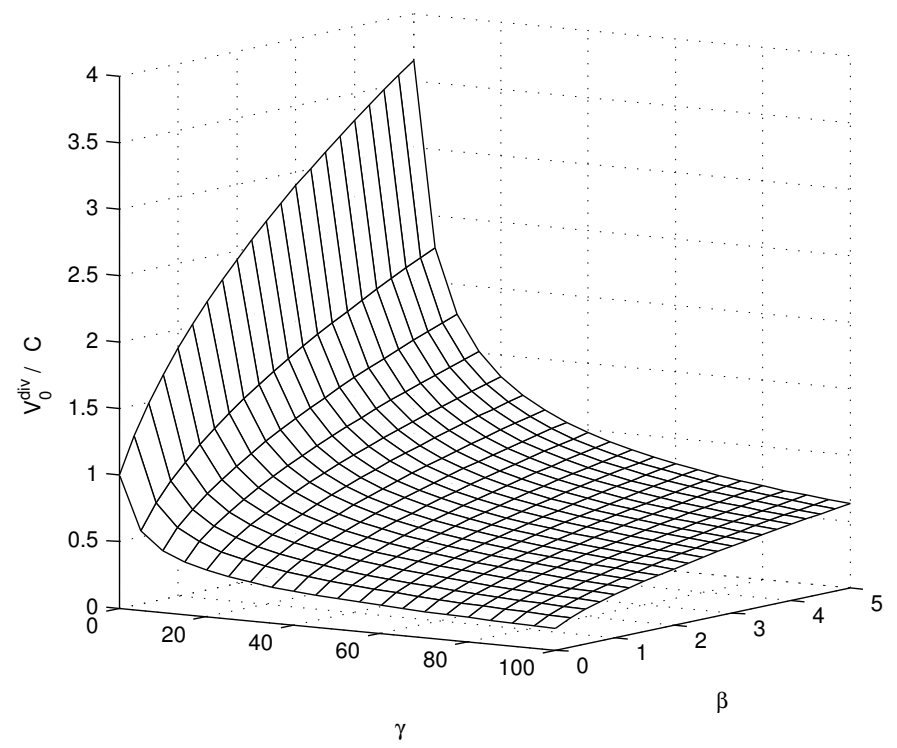

Fig. 6.16 Problem (6.99). Effects of the dimensionless fluid density $\gamma$ and the dimensionless bending rigidity $\beta$ on the dimensionless critical panel velocity $c$. See also slices of this surface in Fig. 6.17.

In Pramila (1986) the nondimensional first natural frequency is given as a function of the nondimensional velocity for some example cases. We will compare the natural frequency predictions of the present model with those of Pramila (1986) later. Currently, we will look at the nondimensional velocity where the natural frequency becomes zero, denoted $c$ in our eigenvalue problem.

From added mass considerations, in Pramila (1986) a scaling factor is obtained. Let us denote this factor by $r$. In the model of Pramila (1986), the first natural frequency and the critical velocity, computed from the vacuum case, both become scaled with the factor $r$, when the ideal fluid is taken into account. In our model, $c$ is the scaling factor for the critical velocity, and the steady-state analysis makes no prediction concerning the natural frequency.

For $m=35.5 \mathrm{~g} / \mathrm{m}^{2}$, Fig. 5 of Pramila (1986, p. 74) suggests that $r$ is in the range $20 \%-30 \%$, depending on which added mass expression is used. Similarly, Fig. 6 of Pramila (1986) suggests that for $m=54 \mathrm{~g} / \mathrm{m}^{2}$, the factor $r$ is in the range $22 \%-35 \%$. For both these examples, the dimensions of the open draw are span length $2 \ell=2.4 \mathrm{~m}$ and web width $b=0.47 \mathrm{~m}$, leading to an aspect ratio of $2 \ell / b \approx 5.1$, i.e. a narrow strip. We will not need the width in our model.

In both cases, we have the dimensionless parameter $\beta=0$. In problem (6.99) $\left(v_{\infty}=V_{0}\right.$, i.e., $\left.\theta=c\right)$, because the value of $T$ only affects the absolute value of $V_{0}^{\text {div }}$ and does not affect $c$, our model predicts that for the first case 

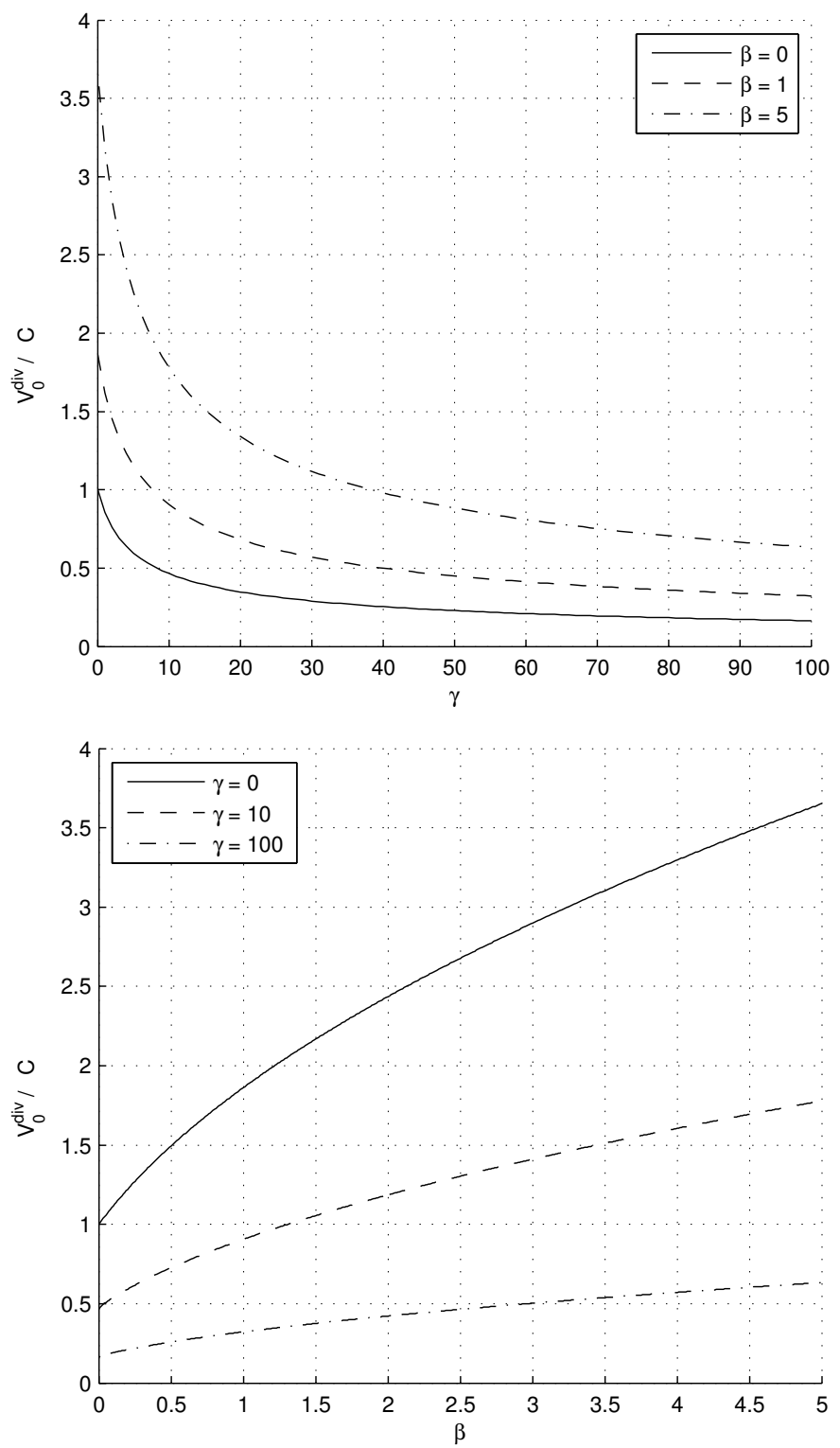

Fig. 6.17 Problem (6.99). Effects of the dimensionless fluid density $\gamma$ and the dimensionless bending rigidity $\beta$ on the dimensionless critical panel velocity $c$. Top: effect of density $\gamma$. Bottom: effect of bending rigidity $\beta$. See also the surface plot in Fig. 6.16. 
$(\gamma=40.5634)$, the factor $r \approx 25.2 \%$ regardless of tension. For the second case $(\gamma=26.6667)$, we have $r \approx 30.6 \%$ regardless of tension. Both cases show good agreement with the results of Pramila (1986).

To illustrate the limitations of the panel model, let us compare the results using another aspect ratio, too. In a numerical example in Pramila (1986), appropriate for comparison here, the geometry is a wide plate with $2 \ell=0.75 \mathrm{~m}$ and $b=7.5 \mathrm{~m}$, giving an aspect ratio of $2 \ell / b=0.1$. The physical parameters are $T=16 \mathrm{~N} / \mathrm{m}$ and $m=50 \mathrm{~g} / \mathrm{m}^{2}$. This gives a vacuum divergence velocity of

$$
C=\sqrt{T / m} \approx 18 \mathrm{~m} / \mathrm{s} .
$$

In Pramila (1986), it is predicted that with these values, depending on the added mass expression used, the critical velocity is found to be between 2.7$4.6 \mathrm{~m} / \mathrm{s}$, or $15 \%-26 \%$ of the vacuum case.

Inserting the numbers to the model presented in this book, we have $\beta=0$ and $\gamma=9.0$. Solving the eigenvalue problem (6.99) gives $V_{0}^{\text {div }} \approx 8.66 \mathrm{~m} / \mathrm{s}$, or $48.4 \%$ of the vacuum case. Here the only agreement is qualitative: according to both models, the divergence velocity decreases when compared to the vacuum case. The quantitative difference is probably due to the deformation localisation effect, which renders the cylindrical deformation assumption invalid in the case of a wide plate. See Fig. 3.5 in Sect. 3.4.

Moving on to the next case, in Fig. 6.18, we have considered the problem of Chang and Moretti (1991) for the critical flow velocity $v_{\infty}^{\text {div }}$, presented in (6.101) above. In this problem, the panel is stationary and is assumed to have zero bending rigidity. The results for $v_{\infty}^{\text {div }}$ from our model, computed by solving (6.101), are shown together with the theoretical results and experimental data of Chang and Moretti (1991). We see that the model presented in this book gives a more accurate prediction for case $\mathrm{A}(20 \times 6 \mathrm{in}, 2 \ell / b \approx 3.33)$ than the original model. However, in case $\mathrm{B}(10 \times 6 \mathrm{in}, 2 \ell / b \approx 1.67)$, the presented model almost systematically overestimates. The critical velocity, according to the measurements, increases more slowly in terms of the applied tension value than predicted. The increasing trend is predicted correctly, but the original model gives a better quantitative approximation. Comparatively better agreement with experiment in case $\mathrm{A}$ is to be expected, since the cylindrical deformation assumption holds best for long and narrow plates (see Fig. 3.5 in Sect. 3.4).

Finally, consider the classical stationary, non-tensioned panel subjected to axial flow. This problem is parameter-free, if we are only interested in the dimensionless $\overline{U_{\mathrm{cd}}}$, so one solution is sufficient. In his book, Païdoussis (2004) states that in the limit of a wide channel, the dimensionless critical flow velocity for a pinned-pinned plate with flow on both sides is

$$
\overline{U_{\text {cd ref }}} \approx 3.3 .
$$

By solving the eigenvalue problem (6.103), we obtain 

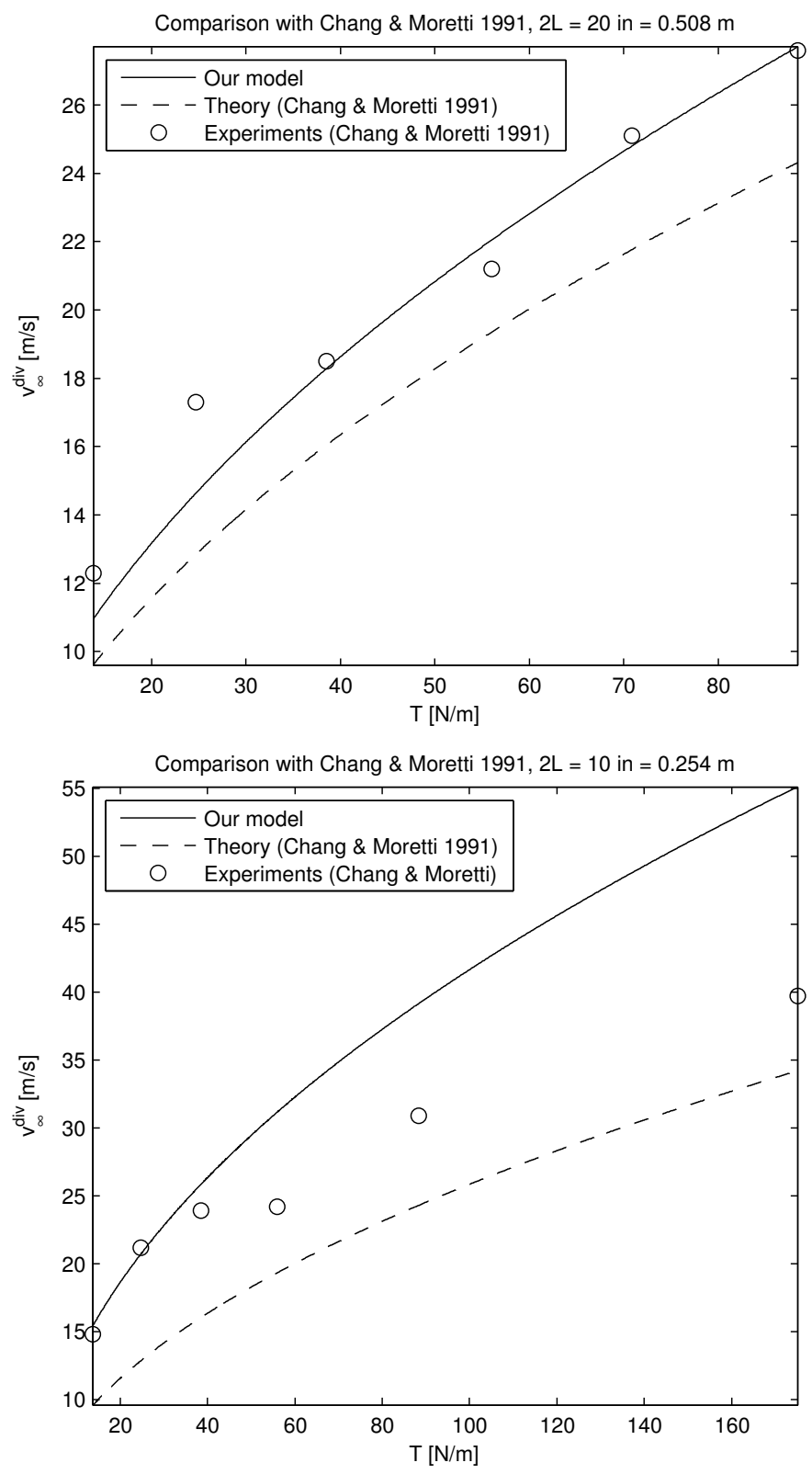

Fig. 6.18 Static stability problem of Chang and Moretti (1991). A stationary panel is subjected to steady-state flow in a wind tunnel; the quantity of interest is the critical flow velocity $v_{\infty}^{\text {div }}$ at a given value of tension T. Top: case A, $2 \ell=0.508 \mathrm{~m}$ (20 in). Bottom: case $\mathrm{B}, 2 \ell=0.254 \mathrm{~m}$ (10 in). 


$$
{\overline{U_{\mathrm{cd}}}}^{2} \approx 2.7788
$$

from which

$$
\overline{U_{\mathrm{cd}}} \approx 1.6670 \text {. }
$$

Our domain is $x \in[-1,1]$, while the standard is $x \in[0,1]$. Hence, we should scale our eigenvalue $\overline{U_{\text {cd }}}$ by 2 to make the results comparable. For an illustration of the issue, consider the eigenvalue problem of the one-dimensional Laplace operator with zero Dirichlet boundary conditions. For

$$
-\frac{\partial^{2} w}{\partial x^{2}}=\lambda^{2} w
$$

the eigenvalues $\lambda$ are inversely proportional to the length of the domain. Hence, if the same problem is solved on a domain that is twice longer, by a different nondimensionalization of $x$, the eigenvalues will be halved. The present problem behaves in a similar manner. Taking this into account produces

$$
2 \overline{U_{\mathrm{cd}}} \approx 3.3339
$$

which agrees with the reference value.

Païdoussis (2004) also lists the quantity ${\overline{U_{\mathrm{cd}}}}^{2} / \pi^{3}$ for several different studies, where flow on one side only has been investigated. In this case

$$
\overline{U_{\text {cd ref }}}
$$

must be scaled by a factor of 2 to account for flow on one side of the plate only (see Païdoussis 2004). This scaling by 2 is independent of the one due to the difference in domain lengths. For our result, we obtain

$$
\left(4 \overline{U_{\mathrm{cd}}}\right)^{2} / \pi^{3} \approx 1.43
$$

which agrees with the value from Païdoussis,

$$
\left(2 \overline{U_{\mathrm{cd} \mathrm{ref}}}\right)^{2} / \pi^{3} \approx 1.4 .
$$

We observe that the choice of $n_{0}$ does not matter. The change in the computed ${\overline{U_{\text {cd }}}}^{2}$ between $n_{0}=1$ and $n_{0}=56$ can be observed to be less than $0.5 \%$. The fast convergence agrees with the remark in the article by Guo and Païdoussis (2000).

Above, the critical velocity of the travelling panel submerged in axial potential flow was investigated numerically as a function of problem parameters. The results were summarized in graphical form. It was found that, quite intuitively, increasing the fluid density decreases the critical velocity, while increasing the bending rigidity increases the critical velocity. The effect of the given free-stream velocity was investigated parametrically, as well as the effects of bending rigidity and fluid density. The numerical examples shown 
give quantitative predictions for the critical velocity when parameter values are given.

A less intuitive result is that, by the model presented in this book, any nonzero free-stream velocity for the fluid decreases the critical velocity of the panel. This is found to be the case even if the fluid and the panel move in the same direction. One would expect any setup with a smaller velocity difference $\left(v_{\infty}-V_{0}\right)$ to resemble more the case of a stationary panel in stationary air; however, as was seen, this is not the case.

In the analysis performed, four different steady-state problems were solved. The first two were related to the critical velocity of the panel, as follows. In the first problem, the free-stream velocity of the fluid $v_{\infty}$ (dimensionless form: $\theta$ ) was given as a problem parameter, while the panel velocity $V_{0}$ (respectively $c$ ) was the unknown eigenvalue.

In the second problem, the whole air mass was assumed to move along with the panel; $v_{\infty}=V_{0}$. In this case, there was only one velocity, which was treated as the unknown eigenvalue $c$. The structure of the equation then became that of a perturbed vacuum model. It was found that if the whole air mass moves with the panel, the critical velocity of the panel is decreased. The decrease is significant; values between $25.2 \%-48.4 \%$ of the corresponding vacuum value were observed.

The other two investigated steady-state problems were related to the flow divergence of stationary panels. As these do not concern axially moving panels, these results are of interest mainly for seeing how the predictions from the model presented in this book differ from known values in literature, when the model is reduced to that of a stationary panel.

Of these, the third problem was the divergence of the stationary tensioned panel when subjected to axial flow. The panel velocity was zero, $V_{0}=0$ (respectively $c=0$ ), and the free-stream velocity of the fluid, $v_{\infty}$ (respectively $\theta$ ), was the unknown eigenvalue. The critical fluid velocity was thus determined. The results were compared to Chang and Moretti (1991). In the case of a long, narrow strip $(20 \times 6$ in, $2 \ell / b \approx 3.33)$, it was seen that the present model improves on the theoretical predictions of Chang and Moretti (1991), in terms of matching experimental data. In the case of a relatively wider span $(10 \times 6$ in, $2 \ell / b \approx 1.67)$, the agreement was not as good, although the qualitative trend was predicted correctly.

The fourth and last problem was the classical non-tensioned stationary panel subjected to axial flow. Again, the panel velocity was zero, $V_{0}=0$ (respectively $c=0$ ), and the free-stream velocity of the fluid, $v_{\infty}$ (respectively $\theta$ ), was the unknown eigenvalue. This was a parameter-free problem with just the dimensionless lowest critical velocity as the solution. The result was compared to those summarized in the book by Païdoussis (2004). The agreement was seen to be good.

To sum up, the parametric study performed in this section gives insight into the behaviour of the critical panel velocity, when fluid-structure interaction is accounted for. The model presented in this book was found to work 
especially well for long, narrow strips. Overall it can be said that the model works well for aspect ratios $2 \ell / b$ of 3 and higher. Qualitative trends are correct for lower aspect ratios, too, but then the critical velocity may be overestimated by the model.

\subsubsection{Dynamical behaviour}

Our next problem is the dynamical behaviour of the system. As above, we will first look into the numerical solution strategy used, and then consider some numerical examples.

For the time integration of (6.93), which is a system of ordinary differential equations, we can proceed using order reduction, and then numerical diagonalization. Let us briefly review these techniques.

Let us truncate the Galerkin series at $n=n_{0}$. The finite equation corresponding to (6.93) is of the general form

$$
\mathbf{M}_{2} \frac{\mathrm{d}^{2} \mathbf{f}(t)}{\mathrm{d} t^{2}}+\mathbf{M}_{1} \frac{\mathrm{d} \mathbf{f}(t)}{\mathrm{d} t}+\mathbf{M}_{0} \mathbf{f}(t)=\mathbf{G}(t),
$$

where the coefficient matrices are defined by the obvious identifications (compare (6.107) and (6.93)), and $\mathbf{G}$ is a vector consisting of the components $G_{j}$ defined by (6.94). For convenience, they are

$$
\begin{aligned}
\left(M_{2}\right)_{j n} & \equiv \alpha^{2}\left[A_{j n}+\gamma a_{j n}\right], \\
\left(M_{1}\right)_{j n} & \equiv 2 \alpha\left[c B_{j n}+\gamma \theta b_{j n}\right], \\
\left(M_{0}\right)_{j n} & \equiv\left(c^{2}-1\right) C_{j n}+\gamma \theta^{2} c_{j n}+\beta D_{j n}, \\
G_{j}\left(t^{\prime}\right) & \equiv \frac{\ell}{T} \int_{-1}^{1} g\left(\ell x^{\prime}, \tau t^{\prime}\right) \Psi_{j}\left(x^{\prime}\right) \mathrm{d} x^{\prime} .
\end{aligned}
$$

In (6.111), the primes explicitly indicate dimensionless quantities. The load function $g$ is the original, dimensional load function (the factor $\ell / T$ then makes $G_{j}$ dimensionless), expressed in the original, unscaled $(x, t)$ coordinates.

System (6.107) can be reduced to a twice larger first-order one by defining

$$
\mathbf{u} \equiv\left[\begin{array}{c}
\mathrm{d} f / \mathrm{d} t \\
\mathbf{f}
\end{array}\right]
$$

where the prime denotes the time derivative. Taking into account that $M_{2}$ is small enough to invert numerically, the expanded equation system becomes

$$
\frac{\mathrm{d}}{\mathrm{d} t}\left[\begin{array}{c}
\mathrm{d} \mathbf{f} / \mathrm{d} t \\
\mathbf{f}
\end{array}\right]=\left[\begin{array}{c}
-\mathbf{M}_{2}^{-1} \mathbf{M}_{1}-\mathbf{M}_{2}^{-1} \mathbf{M}_{0} \\
\mathbf{I}
\end{array}\right]\left[\begin{array}{c}
\mathrm{d} \mathbf{f} / \mathrm{d} t \\
\mathbf{f}
\end{array}\right]+\left[\begin{array}{c}
\mathbf{M}_{2}^{-1} \mathbf{G} \\
0
\end{array}\right],
$$


which can be written as

$$
\frac{\mathrm{d} \mathbf{u}}{\mathrm{d} t}=\mathbf{M u}+\mathbf{g}(t)
$$

where

$$
\mathbf{M}=\left[\begin{array}{cc}
-\mathbf{M}_{2}^{-1} \mathbf{M}_{1} & -\mathbf{M}_{2}^{-1} \mathbf{M}_{0} \\
\mathbf{I} & 0
\end{array}\right], \quad \mathbf{g}(t)=\left[\begin{array}{c}
\mathbf{M}_{2}^{-1} \mathbf{G}(t) \\
0
\end{array}\right] .
$$

The quantities (6.115) are the mass matrix and the force vector of the reduced-order problem.

Standard integration techniques, such as the fourth-order Runge-Kutta method, are directly applicable to the first-order system (6.114). However, we have only one space dimension in the original problem, and the Fourier basis typically does not require many modes along each space dimension in order to get acceptable results. This motivates the use of a diagonalization method (see, e.g., Kreyszig 1993), which can take advantage of the small number of unknowns.

Let us assume that $\mathbf{M}$ has a full eigenvector basis (in practice, this holds). Define $\boldsymbol{\Lambda}, \mathbf{X}, \mathbf{z}$, and $\mathbf{h}(t)$ by

$$
\mathbf{\Lambda} \equiv \mathbf{X}^{-1} \mathbf{M X}, \quad \mathbf{u} \equiv \mathbf{X} \mathbf{z}, \quad \mathbf{X} \mathbf{h}(t) \equiv \mathbf{g}(t),
$$

where $\boldsymbol{\Lambda}$ is a diagonal matrix with the (complex) eigenvalues $\lambda_{j}$ of $\mathbf{M}$ on the diagonal, and $\mathbf{X}$ is a unitary matrix containing the eigenvectors of $\mathbf{M}$ in its columns. Using the relations (6.116), equation (6.114) becomes

$$
\frac{\mathrm{d} \mathbf{z}}{\mathrm{d} t}=\mathbf{\Lambda} \mathbf{z}+\mathbf{h}(t),
$$

The solution for the $j$ th component of $\mathbf{z}$ is (see e.g. Kreyszig 1993)

$$
z_{j}(t)=e^{\lambda_{j} t}\left[z_{j}(0)+\int_{\hat{t}=0}^{t} e^{-\lambda_{j} \hat{t}} h_{j}(\hat{t}) \mathrm{d} \hat{t}\right]
$$

where $\hat{t}$ is a dummy variable for integration and $j=1,2, \ldots, 2 \cdot n_{0}$. The initial value $\mathbf{z}(0)$ is evaluated by using (6.95-6.96) and (6.112), and solving the linear system in (6.116) for $\mathbf{z}$.

Using (6.118), (6.116) and (6.112), the space-discrete solution $\mathbf{f}(t)$ and its time derivative $\mathrm{d} \mathbf{f}(t) / \mathrm{d} t$ may be computed at any desired time $t$ without the need for timestepping. Especially, there is no requirement on the size of the interval of consecutive time values. Because the equation system is small, and $\mathbf{M}$ is constant in time, it is not expensive to compute $\boldsymbol{\Lambda}$ and $\mathbf{X}$ using a standard numerical eigenvalue solver.

Next, let us look into some numerical examples.The Galerkin series is again truncated at $n_{0}=56$, and physical parameters are chosen as typical for a paper web surrounded by air; refer to Table 6.1 on page 165 . The timescale parameter, which is new to the dynamical problem, is chosen as 


$$
\tau=\ell / \sqrt{T / m},
$$

which leads to $\alpha=1$ (see (6.66), p. 155).

Each example is presented in a figure consisting of three parts. The top half displays a space-time plot of the displacement function $w(x, t)$. The horizontal axis represents dimensionless time $t$ and the vertical axis designates the position $x$ between the rollers at $x= \pm 1$ (note the orientation, positive $x$ up). The shade of each point in the image indicates the height, measured from zero displacement.

The bottom half of each figure is made up of two graphs. The graph on the left shows the displacement of the panel as a function of $x$ at a few selected times $t$. The graph on the right shows the time behaviour of the centre point of the panel, $w(0, t)$. The corresponding points in the lower two graphs are marked with circles.

In all of the examples shown, the initial position of the panel is given. For the cases in Figs. $6.19-6.24$, the initial condition for position is

$$
w(x, 0) \equiv g_{1}(x) \equiv a \cdot \sin \left(\pi \frac{x+1}{2}\right),
$$

where the initial amplitude at the center point is $a=5 \cdot 10^{-3}$. The initial transverse velocity is zero,

$$
\frac{\partial w}{\partial t}(x, 0) \equiv g_{2}(x) \equiv 0,
$$

and there are no external disturbances,

$$
g(x, t) \equiv 0 \text {. }
$$

Note that (6.120-6.121) are not fundamental limitations of the model, but are simply choices made to simplify the examples.

In the top subfigures of Figs. $6.19-6.21$, we have as a fundamental test case a plate in vacuum, travelling at various speeds and undergoing a steady, cylindrical vibration. In the space-time plot, the shapes are aligned at an angle to the $x$ axis. Due to the axial motion toward the positive $x$ direction, the positive- $x$ half of the plate experiences each maximum (minimum) of the vibration before the negative- $x$ half does. Physically, as is well known, the wave travelling to the direction of travel on an axially moving medium moves at a higher velocity than the wave travelling in the opposite direction. Mathematically, the phenomenon can be seen as the velocity-dependent phase shift in the eigenmodes of axially travelling strings and beams that was discussed by Wickert and Mote (1990). As was noted in the problem setup, the flat panel model shares the mathematical formulation with the beam model, so we can expect the same phenomenon to occur here.

Let us now move onto the focus of the present study, and consider the effect of fluid-structure interaction. The bottom subfigures of Figs. 6.19-6.21 

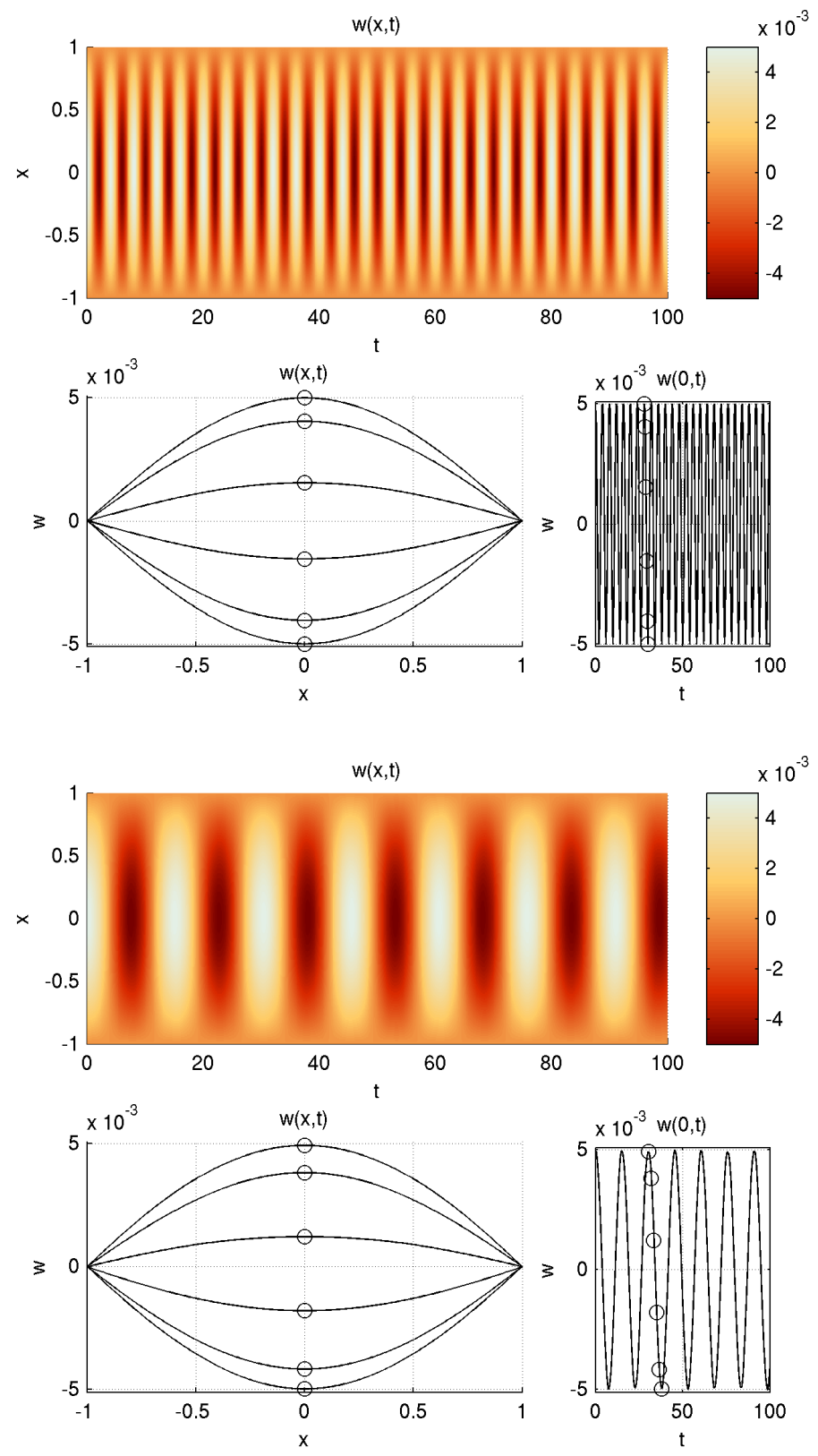

Fig. 6.19 Dynamic response of the panel in vacuum and in stationary fluid. Stationary panel, $V_{0}=0 \mathrm{~m} / \mathrm{s}$. Top: in vacuum. Bottom: submerged in stationary fluid $\left(v_{\infty}=0 \mathrm{~m} / \mathrm{s}\right)$. 

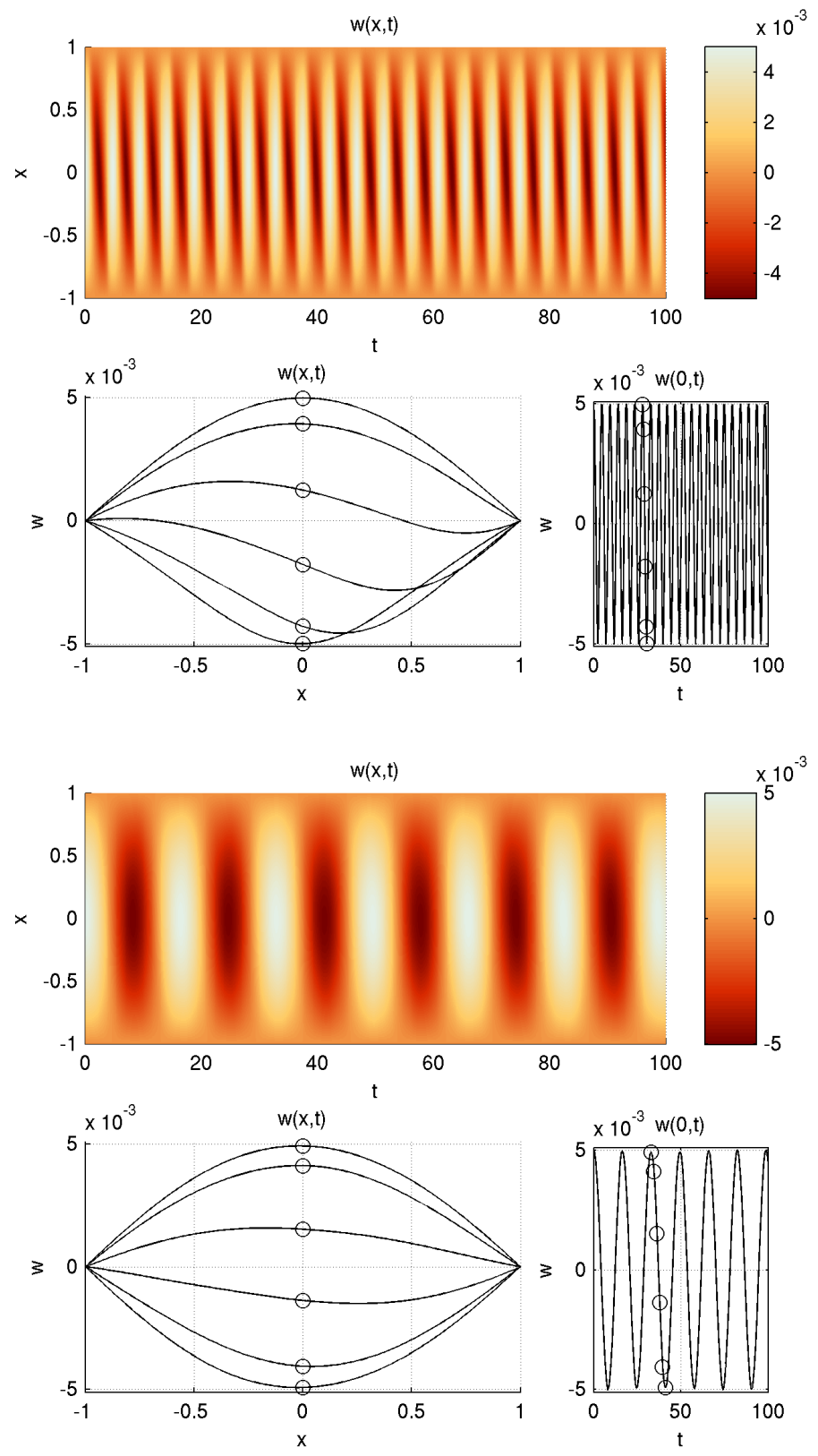

Fig. 6.20 Dynamic response of the panel in vacuum and in stationary fluid. Travelling panel, $V_{0}=30 \mathrm{~m} / \mathrm{s}$. Top: in vacuum. Bottom: submerged in stationary fluid $\left(v_{\infty}=0 \mathrm{~m} / \mathrm{s}\right)$. 

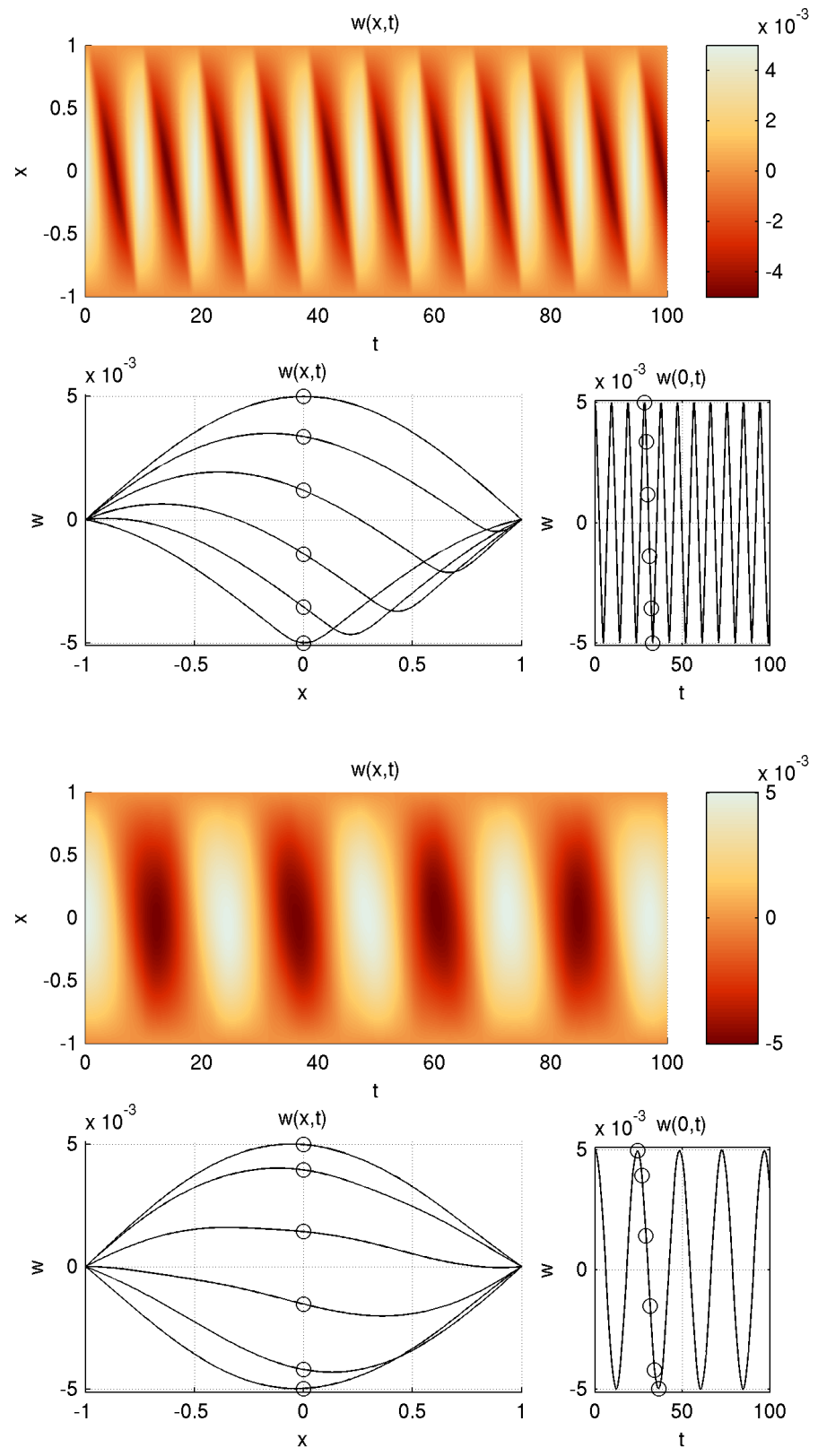

Fig. 6.21 Dynamic response of the panel in vacuum and in stationary fluid. Travelling panel, $V_{0}=60 \mathrm{~m} / \mathrm{s}$. Top: in vacuum. Bottom: submerged in stationary fluid $\left(v_{\infty}=0 \mathrm{~m} / \mathrm{s}\right)$. 

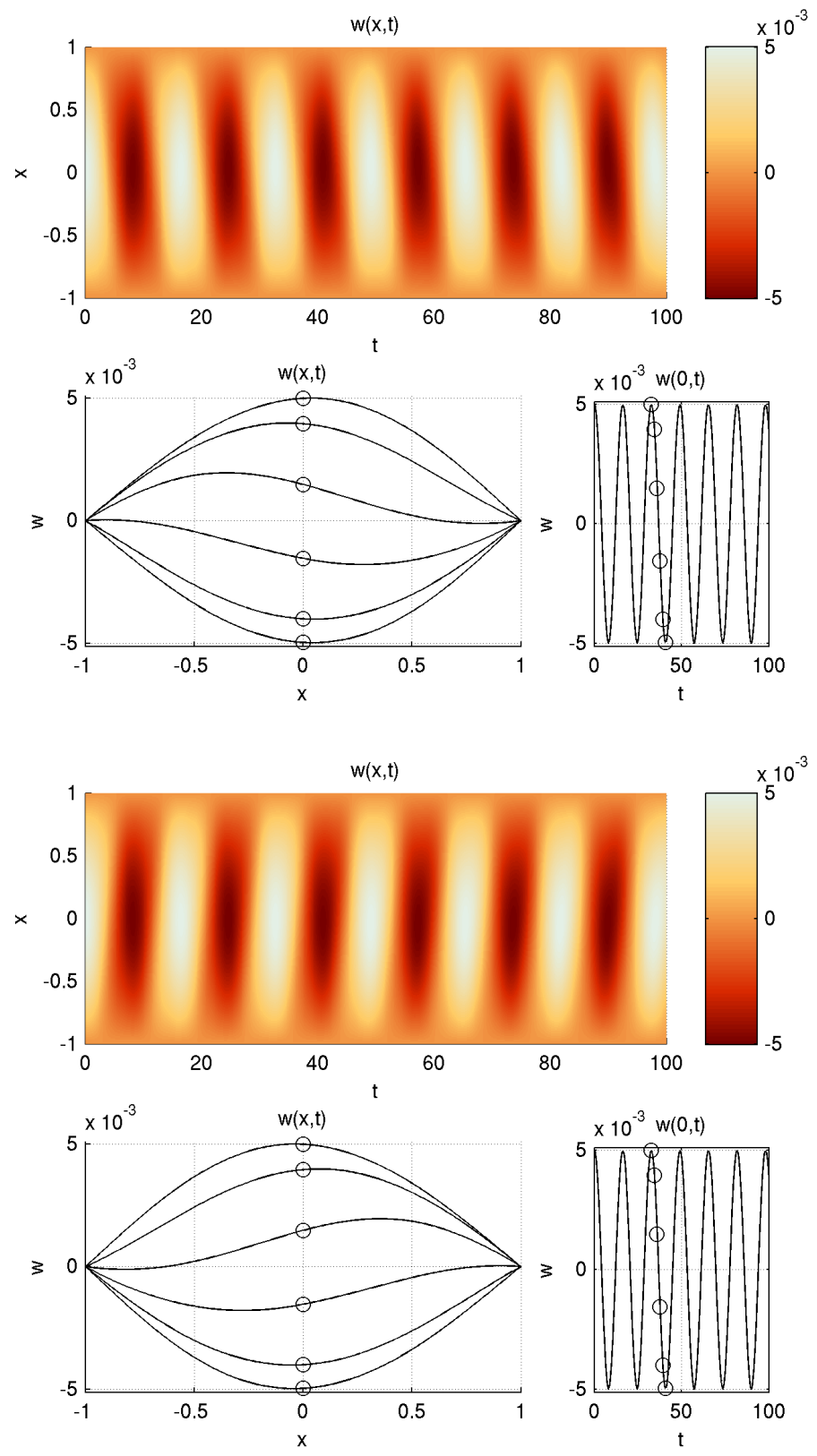

Fig. 6.22 Dynamic response of the panel submerged in potential flow, at some fluid (freestream) and panel velocities. Stationary panel, $V_{0}=0 \mathrm{~m} / \mathrm{s}$. Top: $v_{\infty}=+10 \mathrm{~m} / \mathrm{s}$. Bottom: $v_{\infty}=-10 \mathrm{~m} / \mathrm{s}$. 

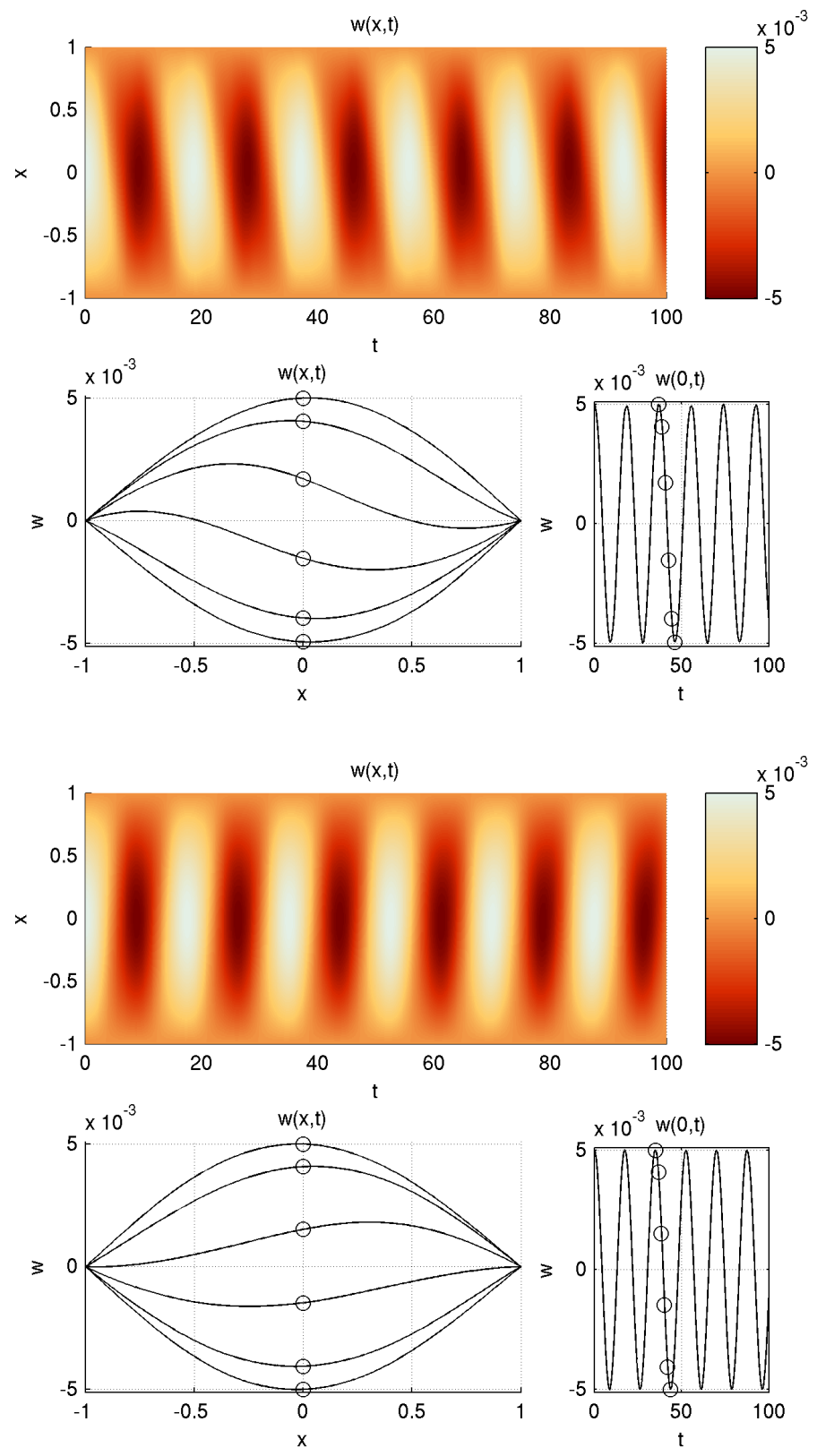

Fig. 6.23 Dynamic response of the panel submerged in potential flow, at some fluid (freestream) and panel velocities. Travelling panel, $V_{0}=30 \mathrm{~m} / \mathrm{s}$. Top: $v_{\infty}=+10 \mathrm{~m} / \mathrm{s}$. Bottom: $v_{\infty}=-10 \mathrm{~m} / \mathrm{s}$. 

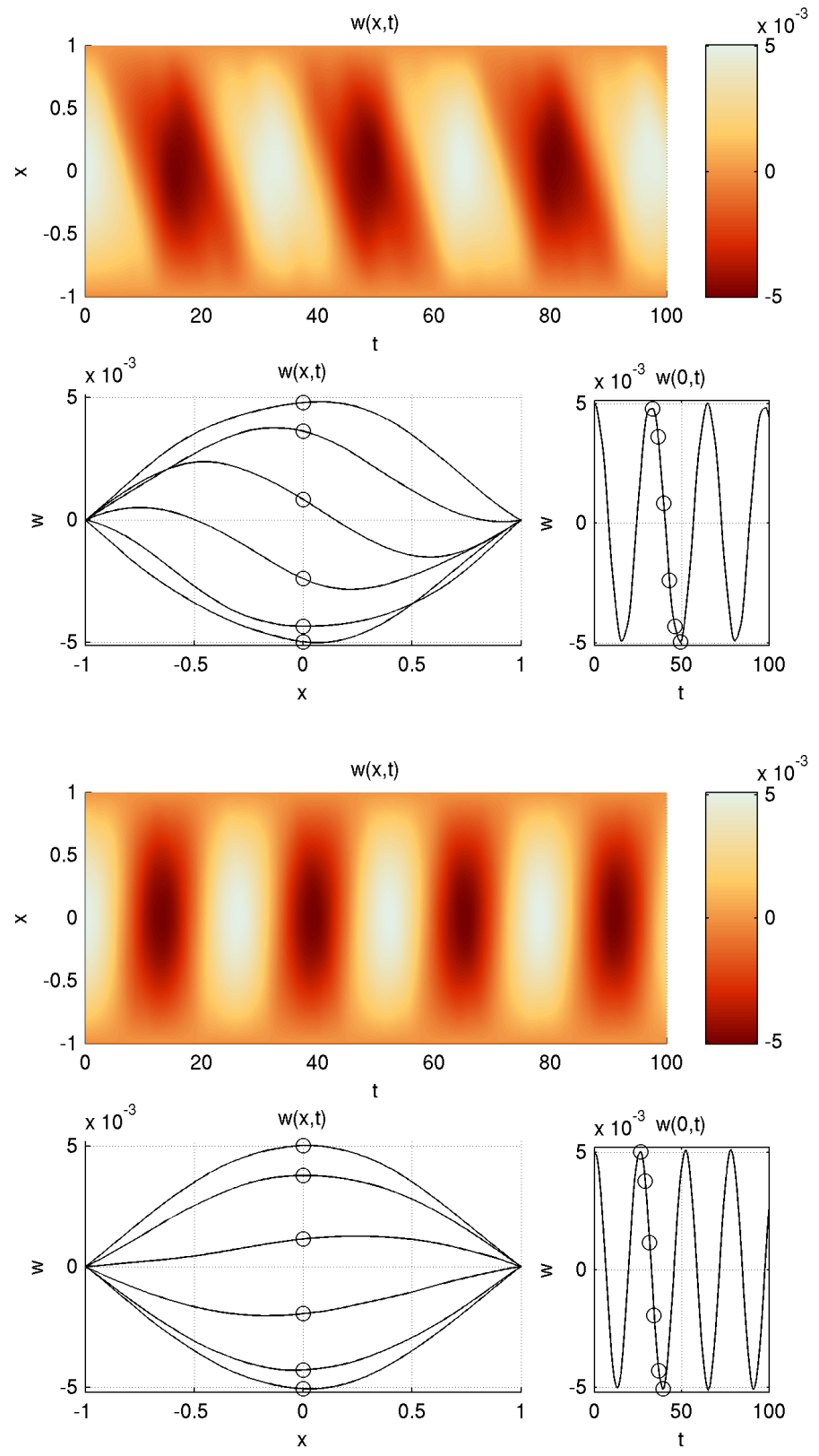

Fig. 6.24 Dynamic response of the panel submerged in potential flow, at some fluid (freestream) and panel velocities. Travelling panel, $V_{0}=60 \mathrm{~m} / \mathrm{s}$. Top: $v_{\infty}=+10 \mathrm{~m} / \mathrm{s}$. Bottom: $v_{\infty}=-10 \mathrm{~m} / \mathrm{s}$. 
represent the dynamic response of the panel in stationary ideal fluid. Figures $6.22-6.24$ represent the response in axially moving fluid. The qualitative behaviour in our Figs. $6.19-6.24$ is seen to be similar to Fig. 2 in Chang et al (1991), where a free vibration cycle of a travelling threadline from a direct simulation was plotted.
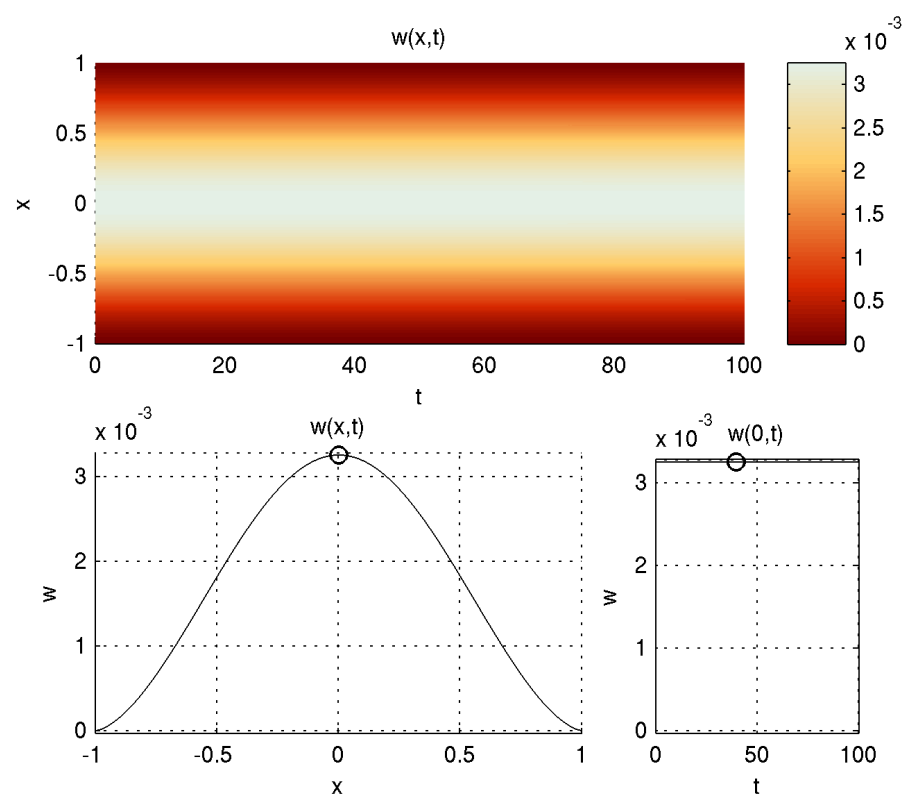

Fig. 6.25 Steady state solution. $V_{0}=70.5257 \mathrm{~m} / \mathrm{s} . v_{\infty}=-15 \mathrm{~m} / \mathrm{s}, t_{\text {fin }}=1 \mathrm{~s}, g_{1}(x)$ set to critical eigenmode, $g(x, t) \equiv 0, g_{2}(x) \equiv 0$.

We conclude these examples with a direct simulation of a special case. The solutions shown in the previous Figs. $6.19-6.24$ are periodic and stable. Figure 6.25 represents the limiting case where a nontrivial static solution (divergence) exists. For this case, the starting position of the panel is specified as the critical eigenmode (buckling mode, divergence mode) of the corresponding steady-state problem.

The steady-state problem can be solved as described above, and the obtained numerical Fourier-Galerkin coefficients and the critical velocity are then used as input data for the dynamic solution process. In this configuration, the initial transverse velocity is zero as per (6.120), and there are no external disturbances, as per (6.121). It is seen, as shown in Fig. 6.25, that the computed solution stays constant in time, as expected.

The following observations can be made about the numerical solutions. First, although the eigenvalues $\lambda_{j}$ are in general complex, the exact solution $\mathbf{u}$ stays real-valued for real-valued initial data. 
The diagonalization method does not cause numerical dissipation of energy, because the qualitative behaviour of the first-order system (6.117) is captured by (6.118).

The initialization of the diagonalization method requires computing $\boldsymbol{\Lambda}$ and $\mathbf{X}$. The asymptotic cost of this initialization is $O\left(n_{0}^{3}\right)$ (with e.g. the $Q R$ algorithm; see Golub and van Loan 1996), so the method is only applicable when the number of unknowns is small. After the initialization, the cost of evaluating the solution at one point of time $t$ is $O\left(n_{0}^{2}\right)$ due to the full vector-matrix multiplication. The advantage of the method is that there is no timestep limitation.

As can be seen from (6.118), any numerical approximation of the transformed solution $\mathbf{z}$ will drift away from the exact one at a rate which depends on the amount of numerical error in the eigenvalues $\lambda_{j}$. The error in the numerical approximation of $\mathbf{u}$ then behaves as a linear combination of these errors, by the transformation (6.116). The error is independent of the spacing of the points of time at which the solution is evaluated.

Numerical error in the eigenvalues may cause small imaginary components to be introduced into the Galerkin coefficients $\mathbf{u}$, which are known a priori to be real. This can be detected by inspecting the imaginary part of the solution at each point of time for which it is computed, and then either ignoring the imaginary part (if small) or stopping computation. In the examples shown, the original solution vector was tested for imaginary parts greater than a threshold. The validity criterion used was $\left|\operatorname{Im}\left(u_{j}\right)\right|<10^{-8}$ separately for each component $j=1,2, \ldots, 2 \cdot n_{0}$. In practice, this criterion was never violated. Then, the small imaginary parts were discarded in visualization (during the assembly of the Galerkin approximation).

Summarizing the above, direct temporal simulations of panel behaviour were made, with and without the effect of the surrounding air. The simulations showed the response of the system as it starts from a given initial position. This gives some intuitive insight into the time evolution of the system.

The examples illustrated were all of the type where the initial position was given and the initial velocity and external disturbances were zero, but the presented approach allows also for given initial velocity and given, dynamic external disturbances (load functions inside the domain).

\subsubsection{Eigenfrequency analysis}

Our final problem in this chapter are the eigenfrequencies of the system. The solution can be obtained as follows. Consider the eigenfrequency problem (free vibration problem) of the space-discrete system (6.107). Let us set the external forces to zero, $\mathbf{G}(t) \equiv 0$, and use the standard time-harmonic trial function: 


$$
\mathbf{f}(t)=\mathbf{F} e^{s t},
$$

where $\mathbf{F}$ is a constant vector. We wish to find all $s \in \mathbb{C}$, and the corresponding nontrivial $\mathbf{F} \in \mathbb{C}^{n_{0}}$, such that

$$
\left[\left(\mathbf{M}_{2} s^{2}+\mathbf{M}_{1} s+\mathbf{M}_{0}\right) \mathbf{F}\right] e^{s t} \equiv \mathbf{L}(s) \mathbf{F} e^{s t}=0 .
$$

The matrices $\mathbf{M}_{j}$ are given by (6.108-6.110), in Sect. 6.2.3.

Obviously, the common exp factor does not matter, so we have

$$
\left(\mathbf{M}_{2} s^{2}+\mathbf{M}_{1} s+\mathbf{M}_{0}\right) \mathbf{F} \equiv \mathbf{L}(s) \mathbf{F}=0 .
$$

To find $s$, we use the determinant method. In order to admit nontrivial $\mathbf{F}$, the matrix $\mathbf{L}(s)$ in (6.123) must be singular. It is easily seen that the zeroes of $\operatorname{det}\left(\mathbf{L}(s)\right.$ ) (as a function of $s$ ) are exactly the eigenvalues of $\mathbf{M}$, the $2 \cdot n_{0} \times 2 \cdot n_{0}$ matrix defined by (6.115). Thus, there are up to $2 \cdot n_{0}$ distinct solutions, all of which can be readily obtained by computing the eigenvalues.

To see the equivalence, expand $\operatorname{det}\left(\mathbf{M}-\lambda_{j} \mathbf{I}\right)$ as a block determinant, noting that the necessary blocks commute. Multiply (6.123) from the left by $\mathbf{M}_{2}^{-1}$. Compare the zero determinant conditions to find that $s=\lambda_{j}$.

The solutions $s$ give us the eigenfrequency spectrum of the system, e.g., for various values of $V_{0}$ with the other parameters kept fixed. The critical velocity at which $s_{j}=0$ for all $j=1,2, \ldots, 2 \cdot n_{0}$, obtained by a numerical search procedure, matches the critical velocity predicted by the static instability analysis.

Finally, it is important to notice that $\mathbf{L}(s) \in \mathbb{C}^{n_{0} \times n_{0}}$, while $\mathbf{M} \in \mathbb{R}^{2 n_{0} \times 2 n_{0}}$ (but with complex eigenvalues). The matrix $\mathbf{L}(s)$ is gyroscopic, because $\mathbf{M}_{2}$ and $\mathbf{M}_{0}$ are symmetric, while $\mathbf{M}_{1}$ is skew-symmetric. The presented method for computing the eigenfrequencies is classical and simple, but other methods also exist specifically for gyroscopic systems. See e.g. Qian and Lin (2007) for a method which works directly on (6.123) without requiring $\mathbf{M}$.

Next, we will briefly review on how to find the eigenvectors $\mathbf{F}$, once the eigenvalues $s$ are known. Consider the matrix $\mathbf{L}(s)$ defined in (6.123), and a fixed $s$, which is a solution of $\operatorname{det}(\mathbf{L}(s))=0$. The singular value decomposition (SVD) is useful here. Write the SVD of the matrix $\mathbf{L}$ as

$$
\mathbf{L}=\mathbf{U S V} \text {, }
$$

where $\mathbf{U}$ and $\mathbf{V}$ are unitary, and $\mathbf{S}$ is a diagonal matrix containing the singular values of $\mathbf{L}$ on the diagonal. Standard numerical solvers can be used to perform the SVD. The algorithmic details, if needed, can be found in the book by Golub and van Loan (1996).

The right-hand side of (6.124) represents a rotation (plus possibly a reflection) $\mathbf{V}$, followed by a scaling $\mathbf{S}$, followed by another rotation (plus possibly reflection) $\mathbf{U}$. In the once-rotated space, the nontrivial kernel of $\mathbf{L}$ must correspond to the zero rows of $\mathbf{S}$, because these rows are then scaled to zero 
before applying the second rotation $\mathbf{U}$. Hence, the question becomes: which vectors in the input space map to the corresponding vector elements?

Let us denote the index set of the zero rows of $\mathbf{S}$ as

$$
I_{0}=\left\{i: S_{i i}=0\right\} .
$$

In this definition, it is sufficient to use just the diagonal elements, because $\mathbf{S}$ is a diagonal matrix. The matrix $\mathbf{L}$ can be viewed as a linear operator in $\mathbb{C}^{n_{0}} \rightarrow \mathbb{C}^{n_{0}}: \mathbf{a} \mapsto \mathbf{L a}$. On the other hand, L $=\mathbf{U S V}$ as was mentioned. Consider the linear mapping

$$
\mathbf{b}=\mathbf{V a},
$$

where $\mathbf{a}$ and $\mathbf{b}$ are (complex) vectors with $n_{0}$ elements. In the next step of applying the product $\mathbf{L}=\mathbf{U S V}$ to the vector $\mathbf{a}$, the elements $\left\{b_{j}: j \in I_{0}\right\}$ of the vector $\mathbf{b}=\left(b_{1}, b_{2}, \ldots, b_{n_{0}}\right)^{\mathrm{T}}$ will be zeroed out in the matrix-vector product $\mathbf{S b}$. Because $\mathbf{V}$ is unitary, multiplying (6.126) from the left by $\mathbf{V}^{\mathrm{H}}$ (the Hermitian conjugate of $\mathbf{V}$ ) gives

$$
\mathbf{V}^{\mathrm{H}} \mathbf{b}=\mathbf{a} .
$$

Since the index set $I_{0}$ can be obtained directly (by inspection of the SVD result), we can choose a set of vectors $\left\{\mathbf{b}_{k}\right\}=\left\{\hat{\mathbf{e}}_{k}: k \in I_{0}\right\}$, where $\hat{\mathbf{e}}_{k}$ is the $k$ th standard basis vector of unit length, and see how each of them maps through (6.127). This procedure gives the corresponding vectors $\mathbf{a}_{k}$ in the original, untransformed space. These vectors represent the directions that are in the null space of $\mathbf{L}$; thus, they are the eigenvectors of the discretized free vibration problem (6.123).

From an implementation viewpoint, computing the SVD is sufficient. The product $\mathbf{V}^{\mathrm{H}} \hat{\mathbf{e}}_{k}$ simply picks the $k$ th column of the matrix $\mathbf{V}^{\mathrm{H}}$. Because $\mathbf{V}^{\mathrm{H}}$ is the Hermitian conjugate of $\mathbf{V}$, this is equivalent with picking the complex conjugate of the $k$ th row of the original $\mathbf{V}$.

The conclusion is that the eigenvectors, corresponding to one fixed eigenfrequency $s$, are

$$
E \equiv\left\{\mathbf{v}_{k}: k \in I_{0}\right\}, \quad \text { where } \quad\left(\mathbf{v}_{k}\right)_{j}=\operatorname{conj}\left(V_{k j}\right),
$$

where $\left(\mathbf{v}_{k}\right)_{j}$ denotes the $j$ th component of the vector $\mathbf{v}_{k}$, and $\operatorname{conj}(\cdot)$ the complex conjugate. The matrix $\mathbf{V}$ comes from the SVD of $\mathbf{L}(s)$, and the index set $I_{0}$ is defined by (6.125). Note that, via the SVD, the set $E$ has the eigenvalue $s$ as a parameter; thus the set of eigenvectors must be evaluated separately for each solution $s$ of the equation $\operatorname{det}(\mathbf{L}(s))=0$.

For the panel problem discussed in this chapter, it occurs in practice that $\# I_{0}=1$, i.e., there is only one eigenvector for each eigenvalue $s$.

Now, let us move on to numerical examples. Examples for the first (lowest) eigenfrequency problem will be discussed first. Then we will discuss examples 
of the eigenfrequency spectrum, which is the final topic in the analysis of our main problems in this chapter.

Let us look at some solutions of the dimensionless first (lowest) eigenfrequency of the system. Let the physical problem parameters be chosen as in Table 6.1 on page 165 , except let the bending rigidity be zero, $D=0$ (membrane limit). As in the dynamic simulations, choose the timescale parameter as

$$
\tau=\ell / C=\ell / \sqrt{T / m},
$$

which leads to $\alpha=1$ (equation (6.66), in Sect. 6.2).

The dimensionless first eigenfrequency of the system is plotted as a function of dimensionless panel velocity $c$ in Fig. 6.26. Two cases are shown: stationary air $\left(v_{\infty}=0\right)$, and the case where the whole air mass moves with the web $\left(v_{\infty}=V_{0}\right)$. Again, the assumption in the second case makes it similar in spirit to the vacuum case, as there is only one independent velocity. It can be seen that the presence of fluid decreases the first natural frequency, as expected (see the studies by Pramila 1986; Frondelius et al 2006; and Kulachenko et al 2007).

The eigenfrequency is non-dimensionalized by $\omega_{0}$, the lowest eigenfrequency of the same system, in vacuum at $V_{0}=0$. The normalization factor can be computed numerically, using the same solution process, but with the fluid terms omitted (effectively setting $q_{\mathrm{f}}(x, t) \equiv 0$ ).

The results are obtained by solving (6.123) as explained above, and taking the lowest dimensionless eigenfrequency as

$$
\omega_{\min }\left(V_{0}\right) \equiv \frac{\min _{j}\left|\operatorname{Im} s_{j}\left(V_{0}\right)\right|}{\omega_{0}} .
$$

For comparison, three pairs of classical analytical results from two addedmass models from a study by Pramila (1987) are included in Fig. 6.26, plotted for our problem parameters. Each pair begins at a single point at $V_{0}=0$, and the different pairs correspond to different aspect ratios (span length per width). In the model of Pramila (1986), the added masses are constants that are affected by $\beta_{\operatorname{Pr}}$, the $\beta$ value of Pramila (1986), which depends on the aspect ratio $R$. For the results shown here, this dependence has been modelled in the form $R\left(\beta_{\operatorname{Pr}}\right)=c_{1} / \beta_{\operatorname{Pr}}+c_{2} / \beta_{\mathrm{Pr}}^{2}$.

To determine the coefficients in the function $R\left(\beta_{\operatorname{Pr}}\right)$, a least squares fit to the data tabulated in Pramila (1986) can be performed using the method of normal equations. The variable $x=\beta_{\mathrm{Pr}}$, and $\left(x_{j}, f_{j}\right)$ are the $\left(\beta_{\mathrm{Pr}}, R\right)$ pairs from Pramila's table. We have used the functions $\phi_{1}(x)=1 / x$ and $\phi_{2}(x)=1 / x^{2}$ as the basis for the least squares fit, obtaining the coefficients $c_{1}=0.1387$ and $c_{2}=0.5318$. Alternative forms, with just the first term $1 / x$, and a three-term form including $1 / x^{3}$, could also be used, but of these the two-term form given here is found to produce the most satisfactory fit.

In Fig. 6.26, the curve marked as eq. (16) corresponds to an added-mass model modifying all three masses (local inertia, Coriolis and centripetal), 
while the one labelled as eq. (22) corresponds to a model modifying only the local inertia mass.

For convenience, equations (16) and (22) from Pramila (1987), converted to our notation, are reproduced below. The real part of the complex eigenfrequency, in both cases, is zero. The imaginary parts are:

$$
\begin{aligned}
& s_{\mathrm{Im}}^{(\mathrm{Pr}, 16)}=\left[1-\frac{\left(m+m_{\mathrm{a}}\right)}{T} V_{0}^{2}\right] \cdot \sqrt{\frac{T}{m+m_{\mathrm{a}}}} \cdot \frac{1}{4 \ell} \\
& s_{\mathrm{Im}}^{(\mathrm{Pr}, 22)}=\frac{1-\frac{m}{T} V_{0}^{2}}{\sqrt{1+\frac{m_{\mathrm{a}}}{m}-\frac{m_{\mathrm{a}}}{T} V_{0}^{2}}} \cdot \sqrt{\frac{T}{m}} \cdot \frac{1}{4 \ell},
\end{aligned}
$$

where the superscript on the left-hand side corresponds to the equation numbering in the reference. We use $4 \ell$ instead of $2 \ell$, because in our notation $\ell$ denotes the half-length of the span. In both equations, (6.130) and (6.131), the added mass $m_{\mathrm{a}}$ is computed as

$$
m_{\mathrm{a}}=2 \ell \rho_{\mathrm{f}} \beta_{\mathrm{Pr}},
$$

where we use $2 \ell$ instead of $\ell$ (as in Pramila 1987) for the same reason as above.

For the equation (16) model, all three added masses are equal. Note that in Pramila (1987), mass per unit length was used, whereas we use mass per unit area. Because the frequency expressions contain only ratios of the parameters, the width can be cancelled out and the results are directly comparable, once the span length and time scaling are taken into account.

We see that the model presented in this book, with $v_{\infty}=0$, agrees closely with equation (22) model of Pramila (1987), as expected. From the discretization (6.93) we see that in this case, only the matrix $a_{j n}$ has an effect. This corresponds in first approximation to a local inertia mass increase. The magnitude of the decrease in eigenfrequency is similar to that reported in Pramila (1986) (about $75 \%)$. For a certain aspect ratio $\left(2 \ell / b \approx 3.1989, \beta_{\operatorname{Pr}}=0.43\right)$, the predictions coincide for the problem parameters used.

In the case $v_{\infty}=V_{0}$, the models qualitatively agree. If we again use $\beta_{\operatorname{Pr}}=$ 0.43 for the model of Pramila (1986), we see that the prediction given by our model for the critical velocity is approximately $60 \%$ higher than that from the corresponding added-mass approach (6.130). On the other hand, if the value $\beta_{\operatorname{Pr}}=0.18$ (corresponding to $2 \ell / b \approx 17.1850$ ) is used, then the predictions of critical velocity agree, but the eigenfrequency given by the added-mass model is approximately $60 \%$ higher. Values of $\beta_{\operatorname{Pr}}$ between these two cases produce results that vary continuously from the first case to the second. See Fig. 6.26 for an example.

It is seen that the primary difference between the present functional approach and the classical results being compared, as far as the lowest eigenfrequency is concerned, is that the predictions change in the case where all 


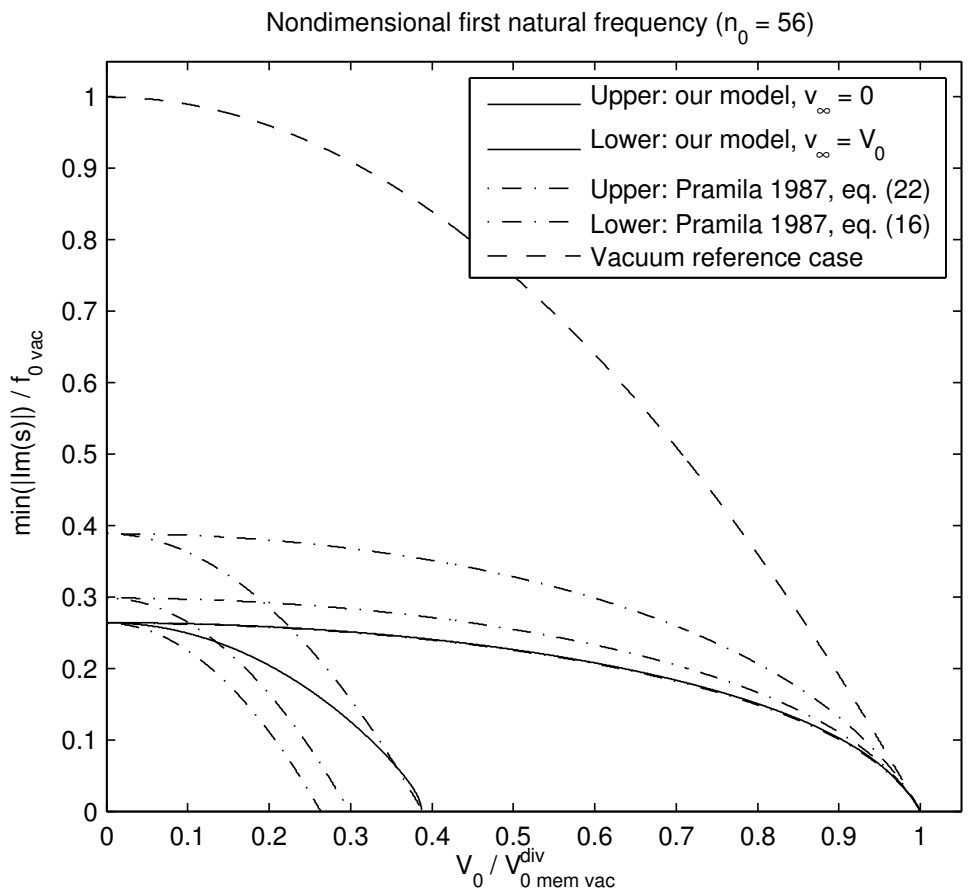

Fig. 6.26 Behaviour of the nondimensional first natural frequency as a function of the nondimensional velocity of the panel. The plot is normalized by the axis intersecting values from the corresponding vacuum case. For the upper solid line, fluid velocity $v_{\infty}=0$. For the lower solid line, $v_{\infty}=V_{0}$, i.e. the air mass moves with the web. For comparison, the vacuum case (dashed line) and three pairs of results corresponding to added-mass formulas (dash-dot lines) from the study by Pramila (1987) are shown. Each pair begins at a single point at $V_{0}=0$. The different pairs correspond to different aspect ratios (length per width). From top to bottom, $2 \ell / b \approx 17.1850\left(\beta_{\mathrm{Pr}}=0.18\right) ; 2 \ell / b \approx 5.1064\left(\beta_{\mathrm{Pr}}=0.3275\right)$; and $2 \ell / b \approx 3.1989\left(\beta_{\operatorname{Pr}}=0.43\right.$; in this particular case, the upper curves coincide). The symbol $\beta_{\operatorname{Pr}}$ refers to the $\beta$ value of Pramila (1986). See also Fig. 6.35 on p. 206 for a view in the $\left(V_{0}, v_{\infty}\right)$ plane. Axis intersection point for $v_{\infty}=V_{0}$ in our model is given as $c^{\mathrm{div}}$ in Table 6.2 (p. 171).

three inertia terms are modified. The aspect ratio never explicitly enters this model, so which prediction differs more, depends on the aspect ratio.

The effect has a simple mathematical explanation. In (6.130), changing the added mass changes the scaling of both axes equally. For each pair of added-mass curves in Fig. 6.26, it is seen that the axis intersection points of the curve corresponding to (6.130) are equal. The present model does not make any such assumption, and thus the scalings, which arise naturally by solving the integro-differential equation, may be different. To summarize, the fact that we may match either the first natural frequency at $V_{0}=0$, or the 


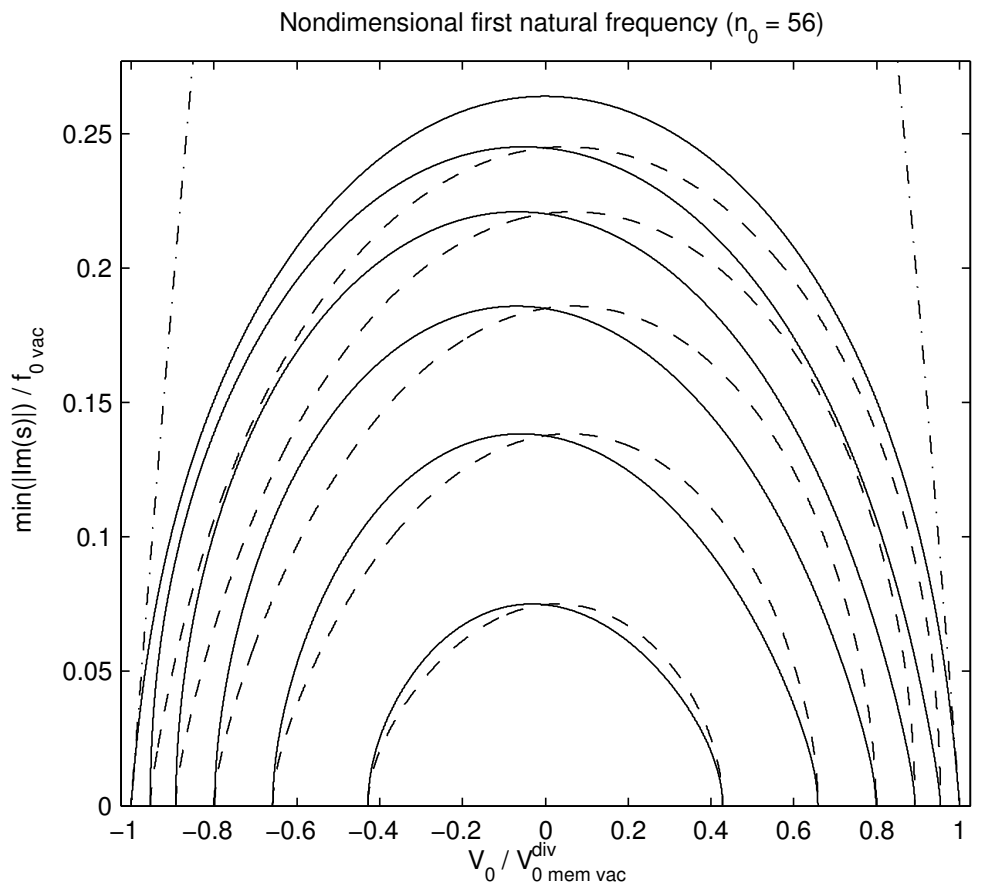

Fig. 6.27 Behaviour of the nondimensional first natural frequency as a function of the nondimensional velocity of the panel. The plot is normalized by the axis intersecting values in vacuum. The vacuum case is partly shown for comparison (dash-dot line). Solid lines indicate $v_{\infty} \geq 0$, dashed lines $v_{\infty}<0$. From top to bottom, the absolute values of the fluid velocities are 0 (corresponds to upper solid line of Fig. 6.26$), 10(\theta \approx 0.13), 15(\theta \approx 0.19)$, $20(\theta \approx 0.25), 25(\theta \approx 0.32)$, and $30 \mathrm{~m} / \mathrm{s}(\theta \approx 0.38)$. Note the scaling of the vertical axis. See also Fig. 6.35 on p. 206 for a view in the $\left(V_{0}, v_{\infty}\right)$ plane. See values $c^{\text {div }}$ in Table 6.2 (p. 171) for the axis intersection points.

critical velocity, but not both, arises from a difference in how mass is handled in the two models.

The equal scaling is not an inherent limitation of the added-mass approach, but is due to the specific form of (6.130). See the study by Chang et al (1991) for a discussion on how different (but still constant) added masses in each term affect the eigenfrequency behaviour. For an approach utilizing boundary layer theory to compute added masses as functions of $x$, also resulting in different scalings for the axes, see Frondelius et al (2006).

In addition to the two classical cases presented in Fig. 6.26, the model presented in this book opens the possibility for studying the problem with an arbitrary axial flow velocity for the surrounding air. In Fig. 6.27, we have plotted first eigenfrequency curves similar to those in Fig. 6.26 for several different fluid velocities $v_{\infty}$. The normalization procedure is the same as in Fig. 6.26. 
Note that as is evident from Fig. 6.27, if $v_{\infty}$ is nonzero and independent of $V_{0}$, the eigenfrequency curves are no longer symmetric with respect to $V_{0}=0$. This reflects the asymmetry in the physics of the situation: the velocities $V_{0}$ and $v_{\infty}$ can have either the same or different signs. We will see by consideration of the higher eigenfrequencies, in Sect. 6.2.5, that the effect of the asymmetry on the eigenfrequency spectrum is much more dramatic than Fig. 6.27 alone suggests. Compare, especially, Figs. $6.29-6.30$ and 6.31 -6.32 below for different signs of $V_{0}$ in the asymmetric case.

\subsubsection{Flutter problem}

Now all that remains is to compute the eigenfrequency spectrum.Let us solve the flutter problem (6.123) for the travelling panel submerged in axial potential flow. As was explained above, we will use the Fourier-Galerkin method for the space discretization.

Because eigenvalue solvers usually return the eigenvalues in random order, a tracking algorithm is needed to construct continuous curves for plotting. Also, solving the discrete system will generally produce numerical artifacts: some solutions will have incorrect asymptotic behaviour (this has been noted e.g. in the study by Parker 1999). These problems have been accounted for in the numerical examples shown; for details, see Jeronen (2011).

To maintain legibility of the plots, we use $n_{0}=10$. The rest of the parameters are the same as for the first eigenfrequency computations. The bending rigidity $D=0$; for the rest, refer to Table 6.1 on page 165 .

No scaling has been applied. The raw data values are shown as-is. Although the 3D plots are shown at an angle to better display their qualitative structure, in each figure the eigenfrequencies start with $\operatorname{Re} s_{j}=0$ (for all $j$ ) at $V_{0}=0$.

We will show both $3 \mathrm{D}$ plots and their projections onto the $\left(V_{0}, \operatorname{Re} s\right)$ and $\left(V_{0}, \operatorname{Im} s\right)$ planes. Both projections are plotted into the same image, with red lines denoting the real parts and blue lines the imaginary parts.

Three cases are considered. First, in Fig. 6.28, the fluid free-stream velocity $v_{\infty}=0$. Second, in Figs. $6.29-6.32$, we have taken $v_{\infty}=20 \mathrm{~m} / \mathrm{s}$. This case is asymmetric in $V_{0}$ due to the physical asymmetry of the situation, as was noted further above; the velocities $V_{0}$ and $v_{\infty}$ may have either the same or different signs. The two figures together cover both situations. Finally, in the third case, $v_{\infty}=V_{0}$, i.e. it is assumed that the whole air mass moves axially with the panel. This situation is shown in Figs. $6.33-6.34$. Compare these plots to the previous Figs. $6.26-6.27$, which show only the first eigenfrequency up to the lowest critical velocity.

The spectra illustrated here suggest that the moving panel with $v_{\infty}=0$, in the membrane limit, has no instability at its first critical point ( $V_{0}$ such that $s=0)$. This is similar to the result by Wang et al (2005) for the classical 
axially moving ideal string. If the free stream of air moves at a given axial velocity $v_{\infty}$, the panel experiences a lowest-mode divergence state at the first critical point for both positive and negative $V_{0}$, even in the membrane limit. This occurs happens already at relatively low free-stream velocities. Although it is not illustrated in the figures, $v_{\infty}=1 \mathrm{~m} / \mathrm{s}$ already exhibits this effect.

In the case where the air mass moves with the web, a lowest-mode divergence state is likewise formed at the first critical point. By the potential flow model, the existence of time-harmonic instability seems to depend on whether the free stream is stationary (in laboratory coordinates) or not.

For this phenomenon, it does not matter whether the free-stream velocity is a separate parameter, or fixed to the panel velocity. This latter phenomenon has a natural mathematical explanation. See Fig. 6.35 for an illustration. In the $\left(V_{0}, v_{\infty}\right)$ plane, fixing $v_{\infty}=V_{0}$ explores a line angled at $45^{\circ}$, passing through the origin. Both velocities are still parameters with given, constant values for each eigenvalue problem solved. Fixing $v_{\infty}$ and varying only $V_{0}$ explores lines angled at $0^{\circ}$ (horizontally) in this same plane. The critical point for the $45^{\circ}$ line corresponds to the critical point of one of the $0^{\circ}$ lines. Similarly, any point at a distance $\varepsilon$ inward from the critical point along this line corresponds to a point in one of the other solutions.

In reality, because of the boundary layer, some of the air indeed moves with the web, so it is reasonable to expect an instability in practice.

Finally, in Figs. 6.36 - 6.39 we include some plots of eigenfunctions $W(x)$ at various $V_{0}$. The plots are normalized such that the maximum complex amplitude is unity, i.e. $\max _{x} W(x) \overline{W(x)}=1$.

In Fig. 6.36, the panel is in vacuum. In Fig. 6.37, stationary ideal fluid is added. In Fig. 6.38 , the fluid moves at $v_{\infty}=20 \mathrm{~m} / \mathrm{s}$. In the final example, Fig. 6.39 , we have set $v_{\infty}=V_{0}$, i.e. the whole air mass moves axially with the panel.

The complex conjugate structure of the eigenfunction pairs is clearly visible in the figures. If $(s, W)$ are an eigenvalue-eigenfunction pair, then so are $(\bar{s}, \bar{W})$. The functions at $V_{0}=0$ are the same for the vacuum case, for $v_{\infty}=0$, and for the case $v_{\infty}=V_{0}$ (which, for $V_{0}=0$, reduces to the previous case). If $v_{\infty}$ is nonzero, the eigenfunctions become different even for $V_{0}=0$ (Fig. 6.38, left; compare the left column of the other three Figs. $6.36-6.37,6.39)$.

In the eigenfrequency analysis above, both the panel and fluid velocities were considered as given problem parameters. Eigenfrequencies were studied parametrically, and some examples of eigenmodes were plotted.

The discrete eigenfrequency spectrum of the system was plotted in three cases: zero free-stream fluid velocity $\left(v_{\infty}=0\right)$, nonzero free-stream fluid velocity (in the example, $v_{\infty}=20 \mathrm{~m} / \mathrm{s}$ was used), and with the whole air mass following the panel $\left(v_{\infty}=V_{0}\right)$. The eigenfrequencies were visualized.

From the present model at the membrane limit $(D=0)$, it was found that if $v_{\infty}=0$, there is no instability, but in all other cases a divergence instability is generated at the lowest critical velocity. 

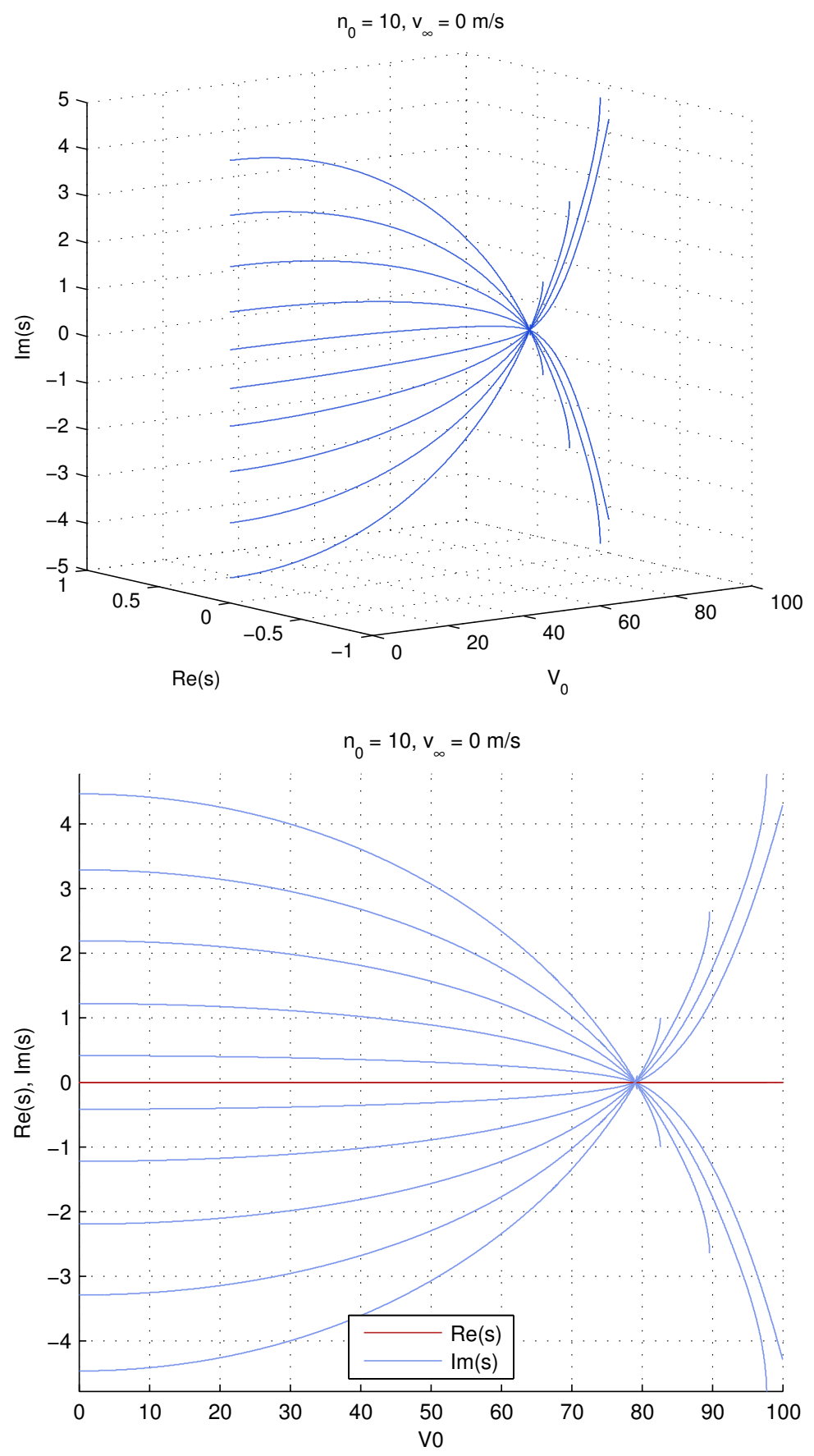

Fig. 6.28 Spectrum of axially moving panel submerged in ideal fluid. Fluid free-stream velocity $v_{\infty}=0$. In the projection, blue line denotes imaginary part, red line real part. Note that $\operatorname{Re} s \equiv 0$. 

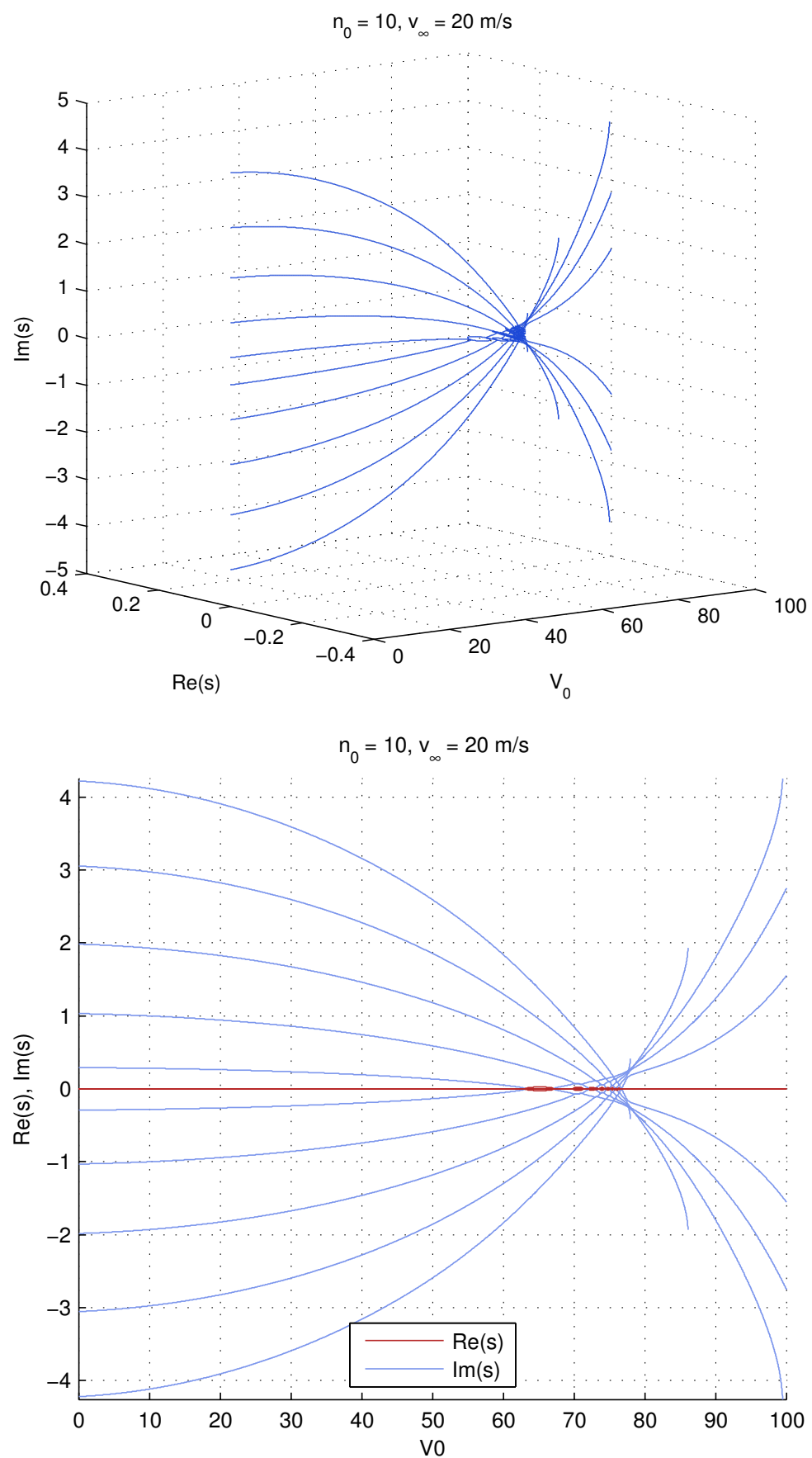

Fig. 6.29 Spectrum of axially moving panel submerged in ideal fluid. Fluid free-stream velocity $v_{\infty}=20 \mathrm{~m} / \mathrm{s}$. Solution for positive $V_{0}$. In the projection, blue line denotes imaginary part, red line real part. 

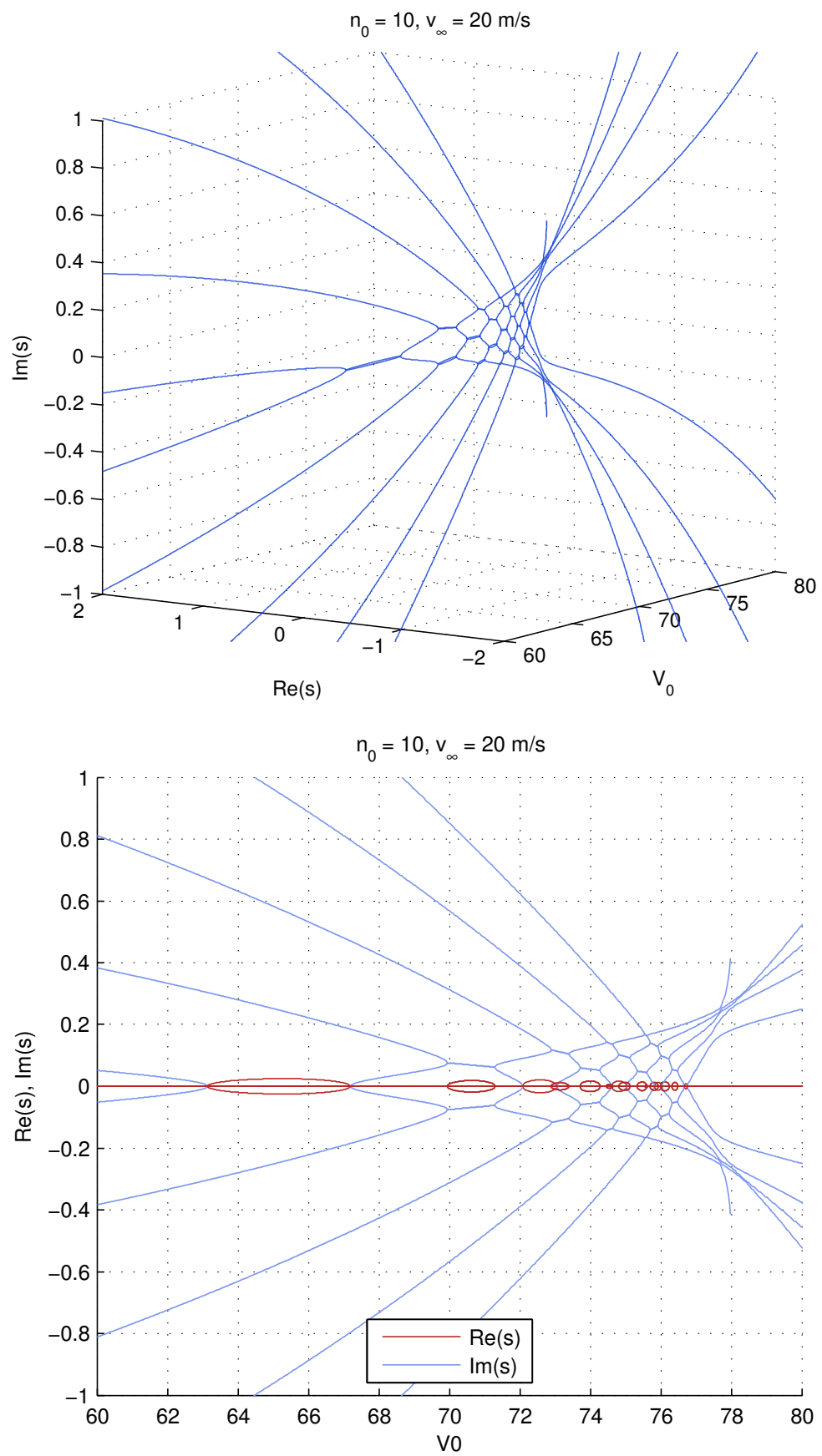

Fig. 6.30 Spectrum of axially moving panel submerged in ideal fluid. Fluid free-stream velocity $v_{\infty}=20 \mathrm{~m} / \mathrm{s}$. Solution for positive $V_{0}$. Detail near critical velocity. In the projection, blue line denotes imaginary part, red line real part. 

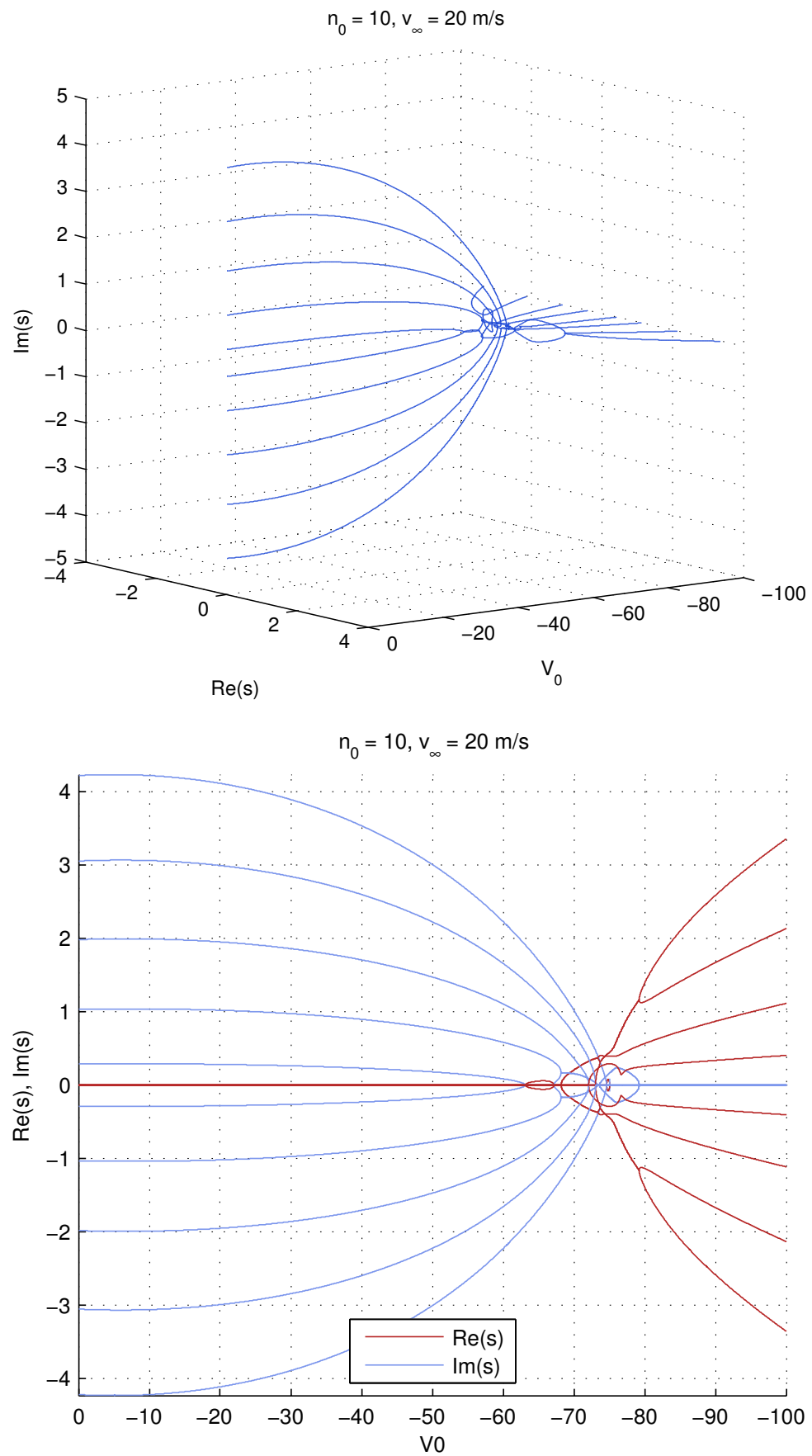

Fig. 6.31 Spectrum of axially moving panel submerged in ideal fluid. Fluid free-stream velocity $v_{\infty}=20 \mathrm{~m} / \mathrm{s}$. Solution for negative $V_{0}$. In the projection, blue line denotes imaginary part, red line real part. 

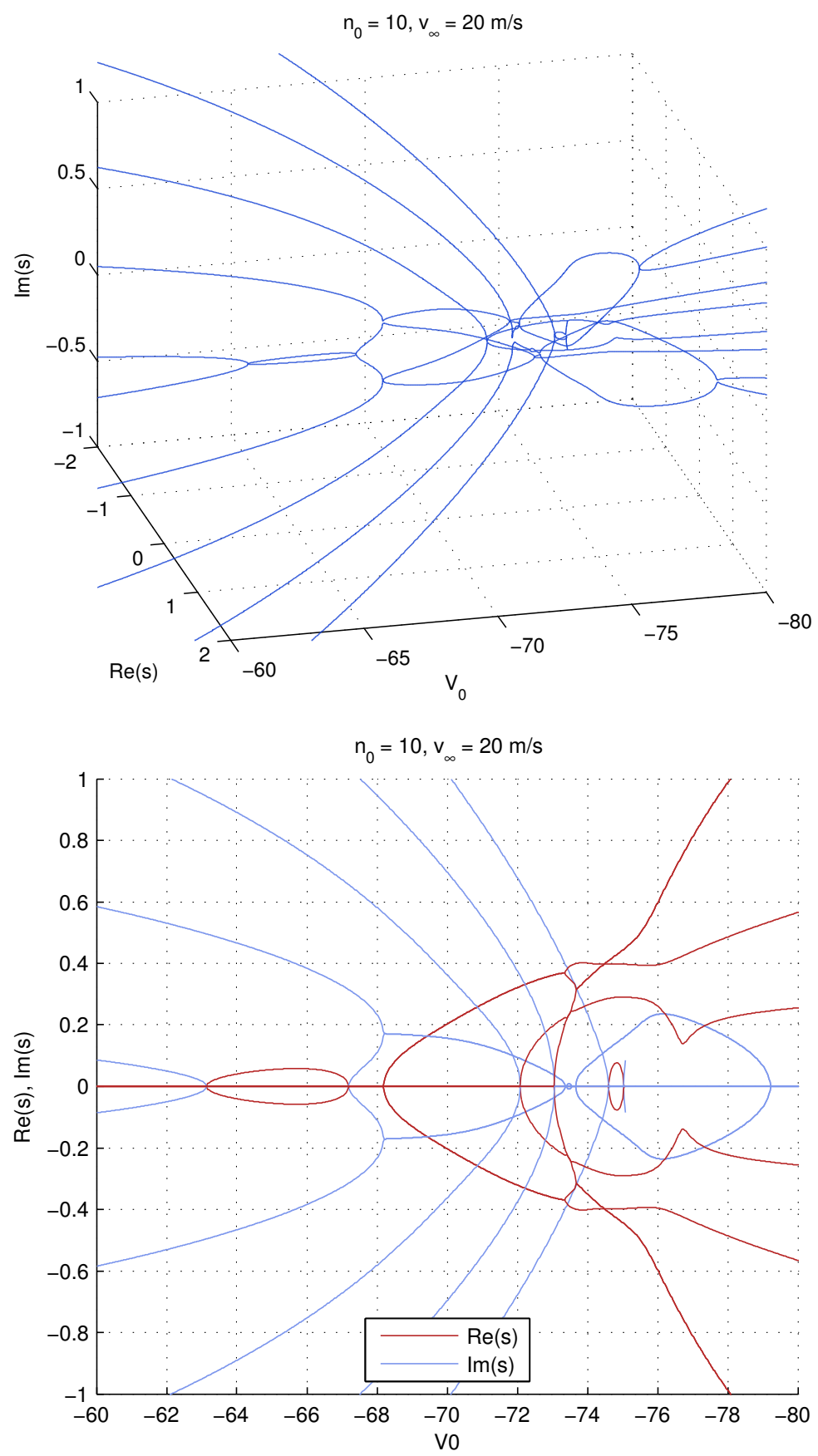

Fig. 6.32 Spectrum of axially moving panel submerged in ideal fluid. Fluid free-stream velocity $v_{\infty}=20 \mathrm{~m} / \mathrm{s}$. Solution for negative $V_{0}$. Detail near critical velocity. In the projection, blue line denotes imaginary part, red line real part. 

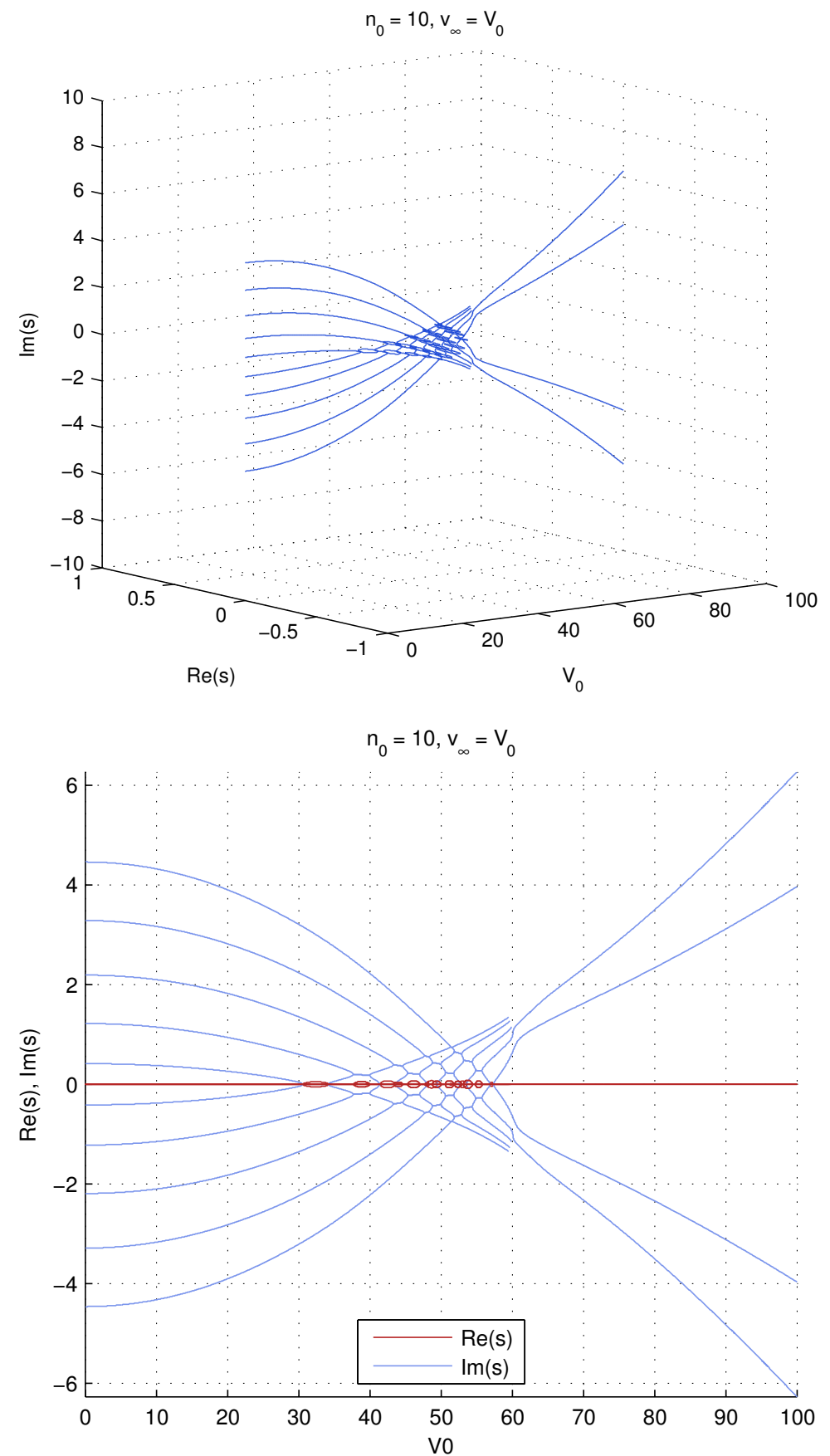

Fig. 6.33 Spectrum of axially moving panel submerged in axially flowing ideal fluid. Fluid free-stream velocity $v_{\infty}=V_{0}$, i.e. whole air mass moves axially with the panel. In the projection, blue line denotes imaginary part, red line real part. 

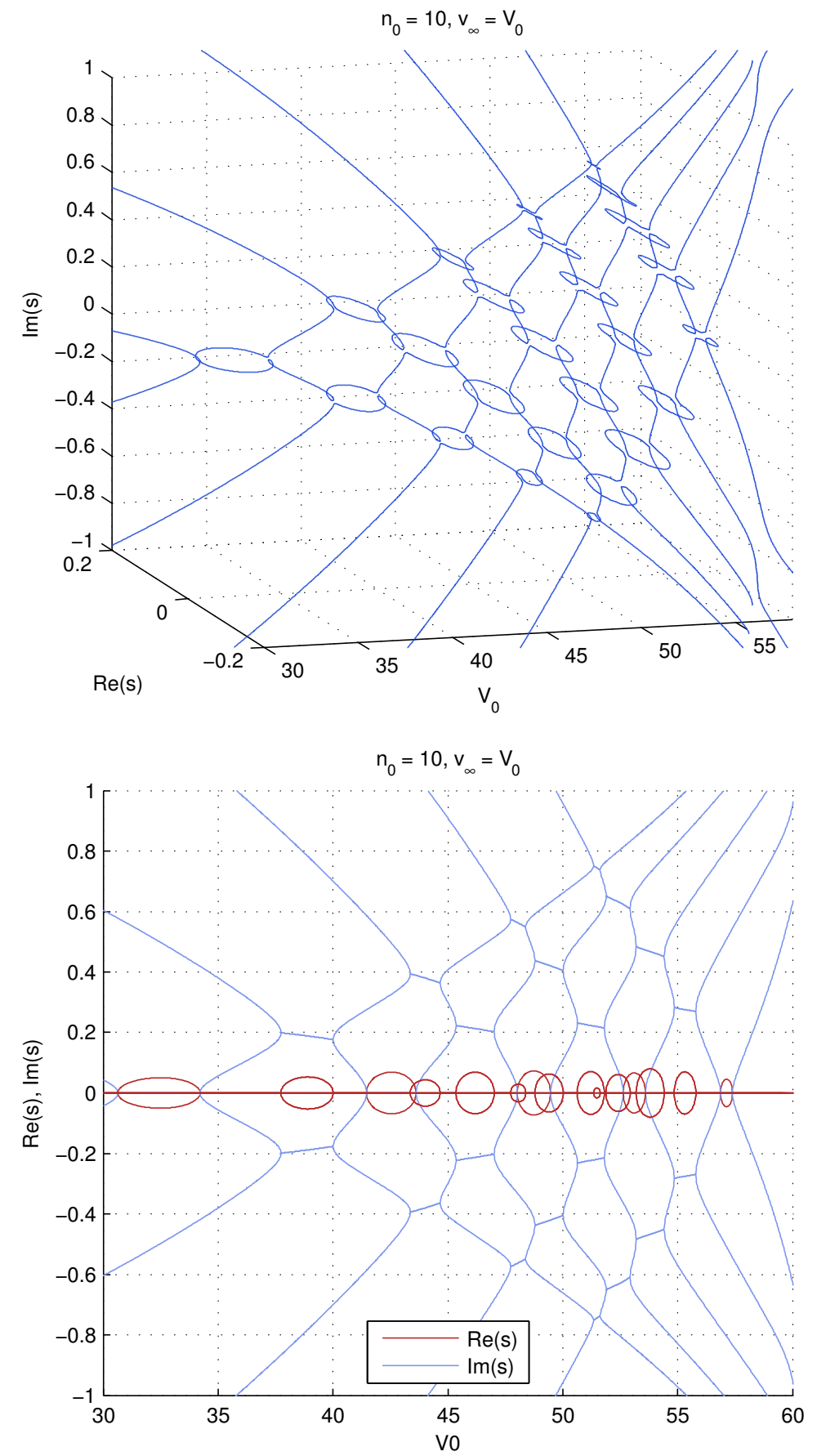

Fig. 6.34 Spectrum of axially moving panel submerged in axially flowing ideal fluid. Fluid free-stream velocity $v_{\infty}=V_{0}$, i.e. whole air mass moves axially with the panel. In the projection, blue line denotes imaginary part, red line real part. Note that the $V_{0}$ grid used for the plot is not dense enough to pick up the eigenvalue collision points in the $3 \mathrm{D}$ view. This makes the elliptical shapes become incomplete toward the right. 


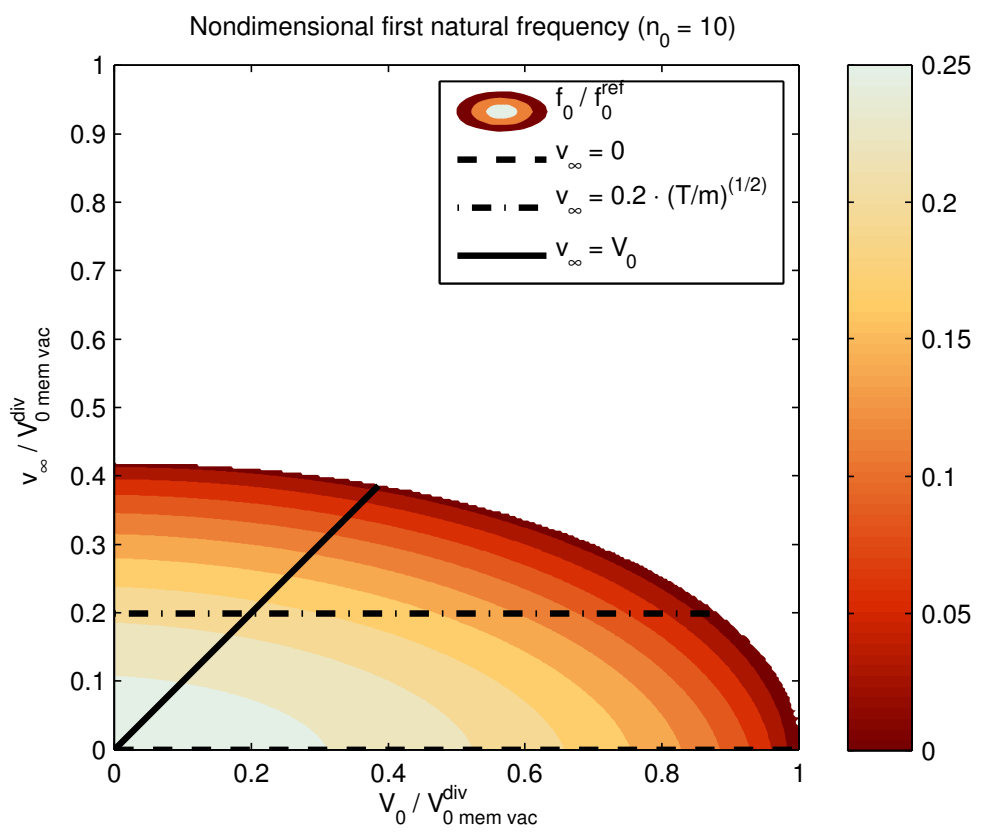

Fig. 6.35 View of the lowest eigenfrequency in the $\left(V_{0}, v_{\infty}\right)$ plane, computed using $n_{0}=10$ modes. When $v_{\infty}$ is a parameter, the spectra (Figs. $6.28-6.32$; also lowest eigenfrequencies in Figs. $6.26-6.27$ ) are taken along horizontal lines in this plane (two examples indicated). When $v_{\infty}=V_{0}$, the spectra (Figs. $6.33-6.34$; also the $v_{\infty}=V_{0}$ case in Fig. 6.26) are taken along the indicated line angled at $45^{\circ}$.

In the case where $v_{\infty}$ has a given nonzero value, it was found that the spectrum becomes asymmetric with respect to reflection of $V_{0}$ through the origin. This is due to the asymmetry in the physics of the situation; the velocities $v_{\infty}$ and $V_{0}$ may have either the same or different signs. Near the critical velocity, the spectrum looks completely different for different signs.

For the case $v_{\infty}=V_{0}$, very nontrivial eigenvalue interaction (Fig. 6.34) was observed in the postcritical regime. However, it should be pointed out that strictly speaking (see e.g. Païdoussis 2005) this is outside the region of validity of the linear model, since an instability exists at the lowest critical velocity.

Returning to the physics, an important qualitative difference was found between classical added-mass models and the present model. As was noted earlier, added-mass models with constant coefficients always behave qualitatively exactly like the corresponding vacuum model. Thus, if there is no bending rigidity (membrane limit), there will be no instability at the critical velocity. In contrast, the introduction of the exact analytical aerodynamic reaction, which changes the form of the equation, may change the stability predictions, by qualitatively changing the behaviour of the eigenfrequency 

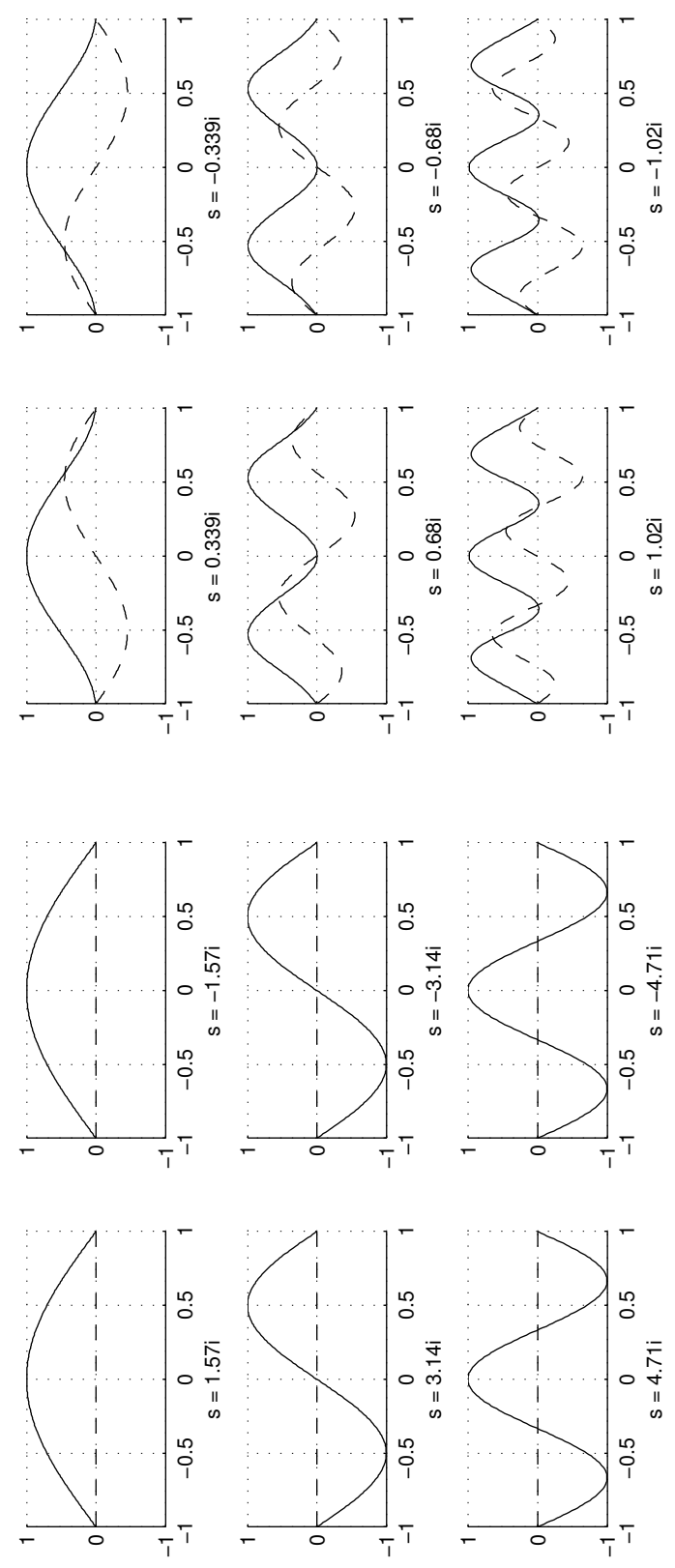

Fig. 6.36 Lowest free vibration eigenmodes of an axially moving panel. Vacuum case. Horizontal axis dimensionless $x$, vertical axis $w(x)$. Solid line is real part, dashed line imaginary part. Bottom: $V_{0}=0$. Top: $V_{0}=70 \mathrm{~m} / \mathrm{s}$ (near critical, $C=\sqrt{T / m}=79.0569 \mathrm{~m} / \mathrm{s}$ ). 

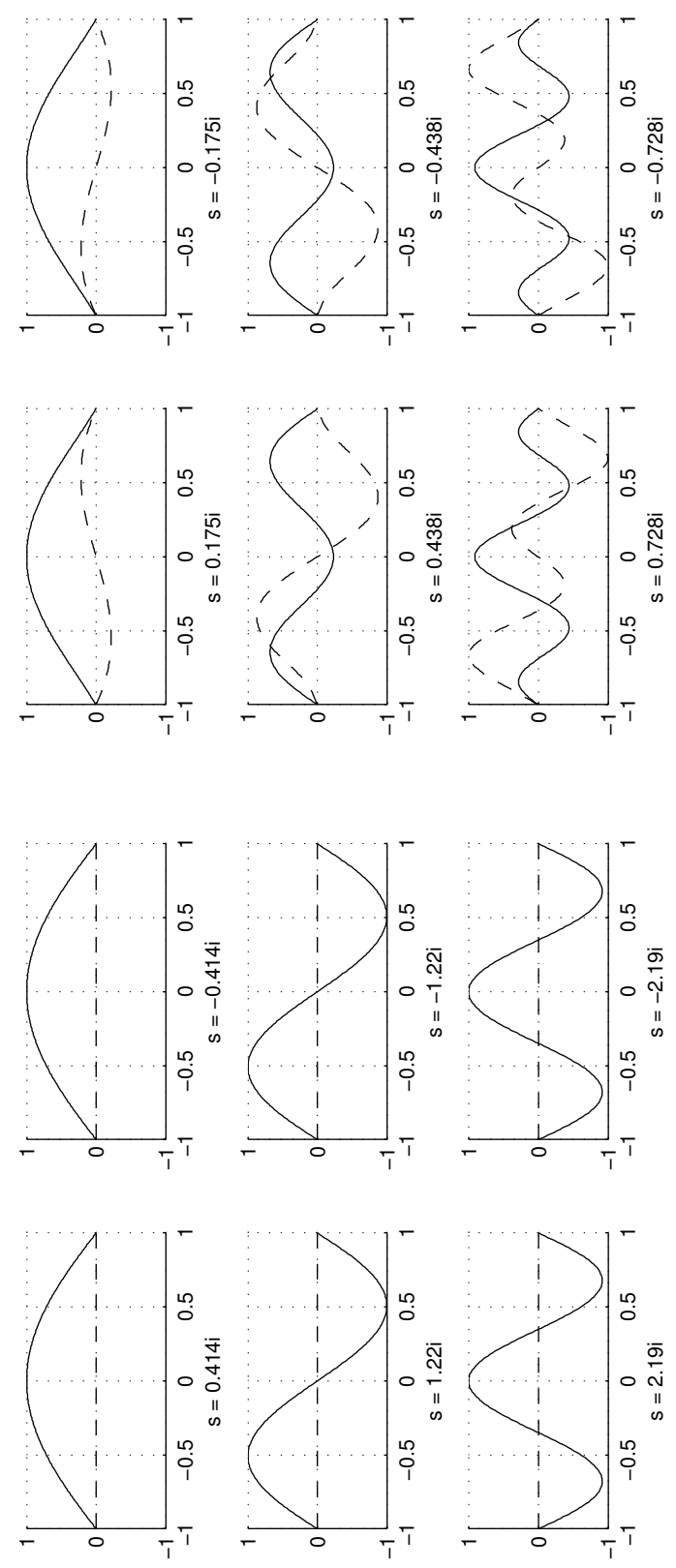

Fig. 6.37 Lowest free vibration eigenmodes of an axially moving panel submerged in ideal fluid. Fluid free-stream velocity $v_{\infty}=0$ (stationary fluid). Horizontal axis dimensionless $x$, vertical axis $w(x)$. Solid line is real part, dashed line imaginary part. Bottom: $V_{0}=0$. Top: $V_{0}=70 \mathrm{~m} / \mathrm{s}$ (near critical, $C=\sqrt{T / m}=79.0569 \mathrm{~m} / \mathrm{s}$ ). 

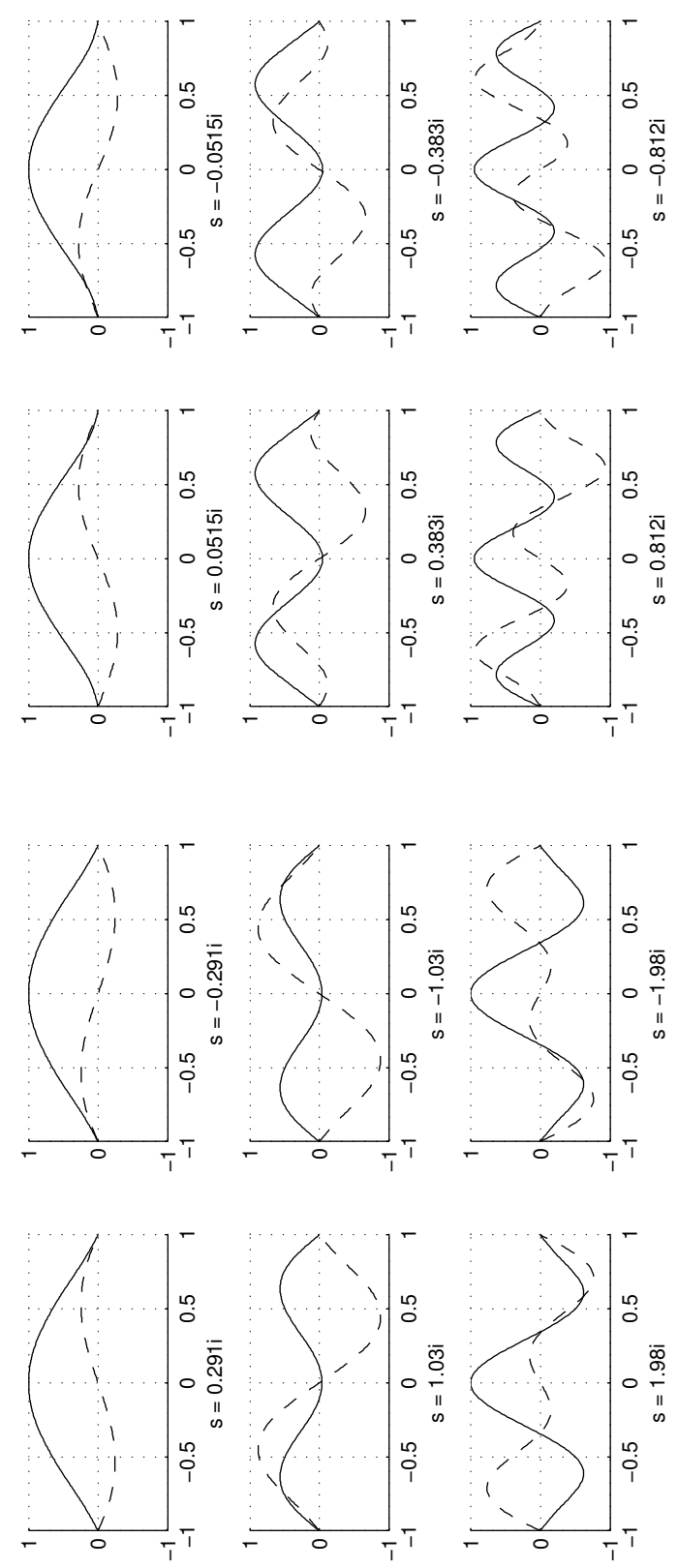

Fig. 6.38 Lowest free vibration eigenmodes of an axially moving panel submerged in axially flowing ideal fluid. Fluid free-stream velocity $v_{\infty}=20 \mathrm{~m} / \mathrm{s}$. Horizontal axis dimensionless $x$, vertical axis $w(x)$. Solid line is real part, dashed line imaginary part. Bottom: $V_{0}=0$. Top: $V_{0}=60 \mathrm{~m} / \mathrm{s}$ (near critical; from steady-state problem, $V_{0}^{\text {crit }} \approx 63.098 \mathrm{~m} / \mathrm{s}$ ). 

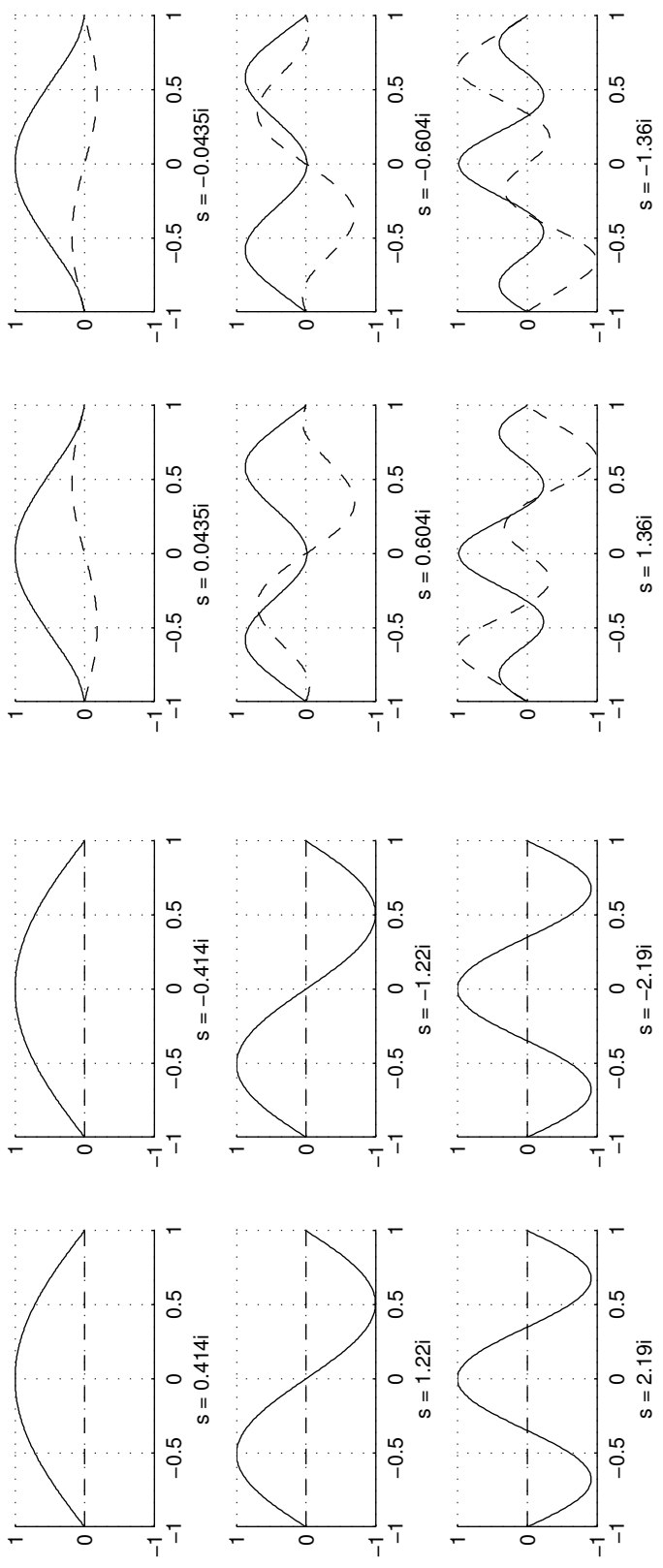

Fig. 6.39 Lowest free vibration eigenmodes of an axially moving panel submerged in axially flowing ideal fluid. Fluid free-stream velocity $v_{\infty}=V_{0}$, i.e. whole air mass moves axially with the panel. Horizontal axis dimensionless $x$, vertical axis $w(x)$. Solid line is real part, dashed line imaginary part. Bottom: $V_{0}=0 . T o p: V_{0}=30 \mathrm{~m} / \mathrm{s}$ (near critical, $\left.V_{0}^{\text {crit }} \approx 30.607 \mathrm{~m} / \mathrm{s}\right)$. 
spectrum of the model. We presented examples of the eigenfrequency spectra at the membrane limit, and indeed observed the introduction of an instability at the lowest critical panel velocity for nonzero free-stream velocities.

In reality, some of the surrounding air will move with the web due to the boundary layer. Thus, it is reasonable to expect that an instability at the critical velocity will occur in practice.

To summarize this chapter, the dynamical behaviour and stability of a moving panel submerged in axial potential flow was considered, with the application of paper production in mind. In any practical paper machine, mechanical stability of the moving paper web is important in order to avoid web breaks, for both technological and economical reasons. Open draws always exist; hence the motion of the web while it travels between supporting rollers is of interest.

The interaction between the travelling material and the surrounding air is especially important for lightweight materials, such as paper. It is not sufficient to consider only the dynamics of the travelling material, but the behaviour of the surrounding airflow must also be analyzed, and its effects on the material fed back into the dynamical model. The coupling changes the dynamics, which, in turn, affects the surrounding flow.

An analytical solution for the airflow problem was derived in terms of the panel displacement, using the model of potential flow. The solution was used in the fluid-structure interaction model to represent the pressure difference across the web in a mathematically exact manner. This had the further advantage of reducing the fluid-structure interaction model into to a single integro-differential equation, saving computational effort.

The fluid-structure interaction problem was studied with simply supported boundary conditions. However, the analytical flow solution is applicable for any boundary conditions for the ends of the moving panel. The presented discretized solution of the moving panel problem can be relatively easily adapted to other Galerkin bases, or to cases with different boundary conditions.

Using Euler's approach for stability analysis and numerical techniques, the critical velocity of the system was obtained. The critical velocity was analyzed numerically as a function of problem parameters, and compared to some existing studies on nearby problems. The predictions from the model can be used to account for the fundamental, physical stability limit in paper machine design.

Direct temporal simulations of the system dynamics were performed to visualize the time evolution of the system, starting from a given initial condition. The spacetime behaviour was visualized, and briefly studied with respect to the web and air velocities. The examples illustrated were all of the type where the initial position was given and the initial velocity and external disturbances were zero, but the presented approach allows also for given initial velocity and given, dynamic external disturbances (load functions inside the domain). 
The complex eigenfrequencies of the system were determined, and studied parametrically as a function of the panel and fluid velocities. By this analysis, it was obtained how the free vibrations of the system behave in different settings. Stability implications were briefly discussed, and it was observed that in the membrane limit, the introduction of the exact analytical aerodynamic reaction qualitatively changes the stability behaviour of the panel model. The results were visualized.

Independent axial motion of the paper web and the free stream were allowed. The model predicted that in all cases, the surrounding air decreases the natural frequencies of the moving panel. Also, if it was assumed that the air mass moves with the web - or if there is a nonzero free-stream velocity - it was seen that the critical velocities of the panel decrease when compared to the corresponding vacuum case.

\section{References}

Allen MB III, Herrera I, Pinder GF (1988) Numerical Modeling in Science and Engineering. Wiley Interscience

Anderson JD Jr (1985) Fundamentals of Aerodynamics. McGraw-Hill

Ashley H, Landahl M (1985) Aerodynamics of wings and bodies. Dover

Bisplinghoff RL, Ashley H (1962) Principles of Aeroelasticity. Dover Publications, Inc., New York, 2nd edition, 1975

Brenner SC, Scott LR (2010) The Mathematical Theory of Finite Element Methods, Texts in Applied Mathematics, vol 15, 3rd edn. Springer

Canuto C, Hussaini MY, Quarteroni A, Zang TA (1988) Spectral Methods in Fluid Dynamics. Springer-Verlag, New York, ISBN 3-540-17371-4

Chang YB, Moretti PM (1991) Interaction of fluttering webs with surrounding air. TAPPI Journal 74(3):231-236

Chang YB, Fox SJ, Lilley DG, Moretti PM (1991) Aerodynamics of moving belts, tapes and webs. In: Perkins NC, Wang KW (eds) ASME DE, vol 36, pp 33-40, presented in ASME Symposium on Dynamics of Axially Moving Continua, Miami, Florida, September 22-25, 1991.

Christou MA, Christov CI (2007) Fourier-Galerkin method for 2D solitons of Boussinesq equation. Mathematics and Computers in Simulation 74:82-92

Ciarlet PG (1978) The Finite Element Method for Elliptic Problems. Studies in Mathematics and its Applications, North-Holland, Amsterdam

Donea J, Huerta A (2003) Finite Element Methods for Flow Problems. Wiley, ISBN: 0-471-49666-9

Eloy C, Souilliez C, Schouveiler L (2007) Flutter of a rectangular plate. Journal of Fluids and Structures 23(6):904-919

Evans LC (1998) Partial Differential Equations. American Mathematical Society, ISBN 0-8218-0772-2 
Frondelius T, Koivurova H, Pramila A (2006) Interaction of an axially moving band and surrounding fluid by boundary layer theory. Journal of Fluids and Structures 22(8):1047-1056

Garziera R, Amabili M (2000) Damping effect of winding on the lateral vibrations of axially moving tapes. ASME Journal of Vibration and Acoustics 122:49-53

Golub GH, van Loan CF (1996) Matrix Computations, 3rd edn. Johns Hopkins, ISBN 0-8018-5414-8

Gresho PM, Sani RL (1999) Incompressible Flow and the Finite Element Method: Advection-Diffusion and Isothermal Laminar Flow. Wiley, reprinted with corrections. ISBN 0471967890

Guo CQ, Païdoussis MP (2000) Stability of rectangular plates with free sideedges in two-dimensional inviscid channel flow. ASME Journal of Applied Mechanics 67:171-176

Hughes TJR (2000) The Finite Element Method. Linear Static and Dynamic Finite Element Analysis. Dover Publications, Inc., Mineola, N.Y., USA, ISBN 0-486-41181-8

Jeronen J (2011) On the mechanical stability and out-of-plane dynamics of a travelling panel submerged in axially flowing ideal fluid: a study into paper production in mathematical terms. PhD thesis, Department of Mathematical Information Technology, University of Jyväskylä, URL http://julkaisut.jyu.fi/?id=978-951-39-4596-1, Jyväskylä studies in computing 148. ISBN 978-951-39-4595-4 (book), ISBN 978-951-39-4596-1 (PDF)

Johnson C (1987) Numerical Solution of Partial Differential Equations by the Finite Element Method. Cambridge University Press, reprint by Dover, 2009 .

Kornecki A, Dowell EH, O'Brien J (1976) On the aeroelastic instability of two-dimensional panels in uniform incompressible flow. Journal of Sound and Vibration 47(2):163-178

Kreyszig E (1993) Advanced Engineering Mathematics, 7th edn. John Wiley \& Sons, ISBN 0-471-59989-1

Krizek M, Neittaanmäki P (1990) Finite Element Approximation of Variational Problems and Applications. Longman Scientific \& Technical, Harlow, copubl. J. Wiley \& Sons, New York

Kulachenko A, Gradin P, Koivurova H (2007) Modelling the dynamical behaviour of a paper web. Part II. Computers \& Structures 85:148-157

Lighthill J (1986) An Informal Introduction to Theoretical Fluid Mechanics. Oxford Science Publications, ISBN 0-19-853630-5

Nehari Z (1952) Conformal Mapping. Dover, ISBN 0-486-61137-X

Païdoussis MP (2004) Fluid-Structure Interactions: Slender Structures and Axial Flow, vol 2. Elsevier Academic Press, ISBN 0-12-544361-7

Païdoussis MP (2005) Some unresolved issues in fluid-structure interactions. Journal of Fluids and Structures 20(6):871-890 
Païdoussis MP (2008) The canonical problem of the fluid-conveying pipe and radiation of the knowledge gained to other dynamics problems across applied mechanics. Journal of Sound and Vibration 310:462-492

Parker RG (1999) Supercritical speed stability of the trivial equilibrium of an axially-moving string on an elastic foundation. Journal of Sound and Vibration 221(2):205-219

Pramila A (1986) Sheet flutter and the interaction between sheet and air. TAPPI Journal 69(7):70-74

Pramila A (1987) Natural frequencies of a submerged axially moving band. Journal of Sound and Vibration 113(1):198-203

Qian J, Lin WW (2007) A numerical method for quadratic eigenvalue problems of gyroscopic systems. Journal of Sound and Vibration 306(1-2):284296, URL http://dx.doi.org/10.1016/j.jsv.2007.05.009

Sedov LI (1972) A Course in Continuum Mechanics, vol 3, English edn. Wolters-Noordhoff Publishing, Groningen, Netherlands, ISBN 90-01796826

Sherman DI (1952) On the stress distribution in partitions, an elastic heavy medium which is weakened by elliptic holes. Izvestiya Akademii Nauk SSSR, Otdelenie Tekhnicheskikh Nauk (OTN) 7:992-1010

Silberman I (1954) Planetary waves in the atmosphere. Journal of Meteorology 11:27-34

Strang WG, Fix GJ (1973) An Analysis of the Finite Element Method. Wellesley Cambridge Press, ISBN 978-0961408886

Tadmor E (1987) Stability analysis of finite-difference, pseudospectral and Fourier-Galerkin approximations for time-dependent problems. SIAM Review 29(4):525-555

Wang Y, Huang L, Liu X (2005) Eigenvalue and stability analysis for transverse vibrations of axially moving strings based on Hamiltonian dynamics. Acta Mechanica Sinica 21:485-494

Wickert JA, Mote CD (1990) Classical vibration analysis of axially moving continua. ASME Journal of Applied Mechanics 57:738-744 


\title{
Chapter 7 Fracture and fatigue of travelling plates
}

\begin{abstract}
In this chapter, problems of fracture and stability of a moving plate, travelling in a system of rollers at a constant velocity, are studied. It is known that in the manufacturing process, there may occur many kinds of defects in the paper web, such as edge cracks and blister and fiber cuts. Our aim is to tackle this problem and analyse theoretically how the defects change the behaviour. We will use the model of a thin elastic plate made of brittle material. A plate with initial cracks is studied, subjected to constant tension and cyclic tension. As a result, we will show how to find safe parameter ranges of transport velocities and in-plane tensions when fracture, stability and constraints on longevity are taken into account.
\end{abstract}

\subsection{Travelling plates under fracture and instability constraints}

In the following sections, we will discuss fracture of travelling rectangular plates based on the theory of linear elastic fracture mechanics. Two different cases of tension profiles will be considered: homogeneous tension and linearly distributed nonhomogeneous tension. With the help of analytical expressions, we will present an optimal value for the plate velocity.

\subsubsection{Safe range of velocities for the case of homogeneous tension}

Consider a rectangular, elastic plate travelling at a constant velocity $V_{0}$ in the $x$ direction between rollers located at $x=0$ and $x=\ell$. See Fig. 7.1. In a cartesian coordinate system, the plate spans the region 


$$
\Omega \equiv\left\{(x, y) \in \mathbb{R}^{2} \mid 0<x<\ell,-b<y<b\right\} .
$$

The quantities $\ell$ and $b$ are considered given. We will model the plate as a rectangular, isotropic elastic plate having constant thickness $h$, Poisson ratio $\nu$, Young modulus $E$ and bending rigidity $D$.

The plate is assumed to be subjected to constant tension $T_{0}$, acting in the $x$ direction. The sides of the plate

$$
\Gamma_{\ell}=\{x=0,-b \leq y \leq b\} \quad \text { and } \quad \Gamma_{r}=\{x=\ell,-b \leq y \leq b\}
$$

are simply supported, and the sides

$$
\Gamma_{-}=\{y=-b, 0 \leq x \leq \ell\} \quad \text { and } \quad \Gamma_{+}=\{y=b, 0 \leq x \leq \ell\}
$$

are free of tractions.

Stationary equations describing the behaviour of the plate with the applied boundary conditions form the following eigenvalue problem (which is a buckling problem):

$$
\begin{gathered}
\left(m V_{0}^{2}-T_{0}\right) \frac{\partial^{2} w}{\partial x^{2}}+D\left(\frac{\partial^{4} w}{\partial x^{4}}+2 \frac{\partial^{4} w}{\partial x^{2} \partial y^{2}}+\frac{\partial^{4} w}{\partial y^{4}}\right)=0, \quad \text { in } \Omega, \\
w=0, \quad \frac{\partial^{2} w}{\partial x^{2}}=0, \quad \text { on } \Gamma_{\ell} \text { and } \Gamma_{r}, \\
\frac{\partial^{2} w}{\partial y^{2}}+\nu \frac{\partial^{2} w}{\partial x^{2}}=0, \quad \text { on } \Gamma_{-} \text {and } \Gamma_{+}, \\
\frac{\partial^{3} w}{\partial y^{3}}+(2-\nu) \frac{\partial^{3} w}{\partial x^{2} \partial y}=0, \quad \text { on } \Gamma_{-} \text {and } \Gamma_{+},
\end{gathered}
$$

where $D=E h^{3} /\left(12\left(1-\nu^{2}\right)\right)$, and $m$ is the mass per unit area of the plate. We denote the eigenvalue

$$
\lambda=\gamma^{2}=\frac{\ell^{2}}{\pi^{2} D}\left(m V_{0}^{2}-T_{0}\right) .
$$

As was discussed in Chap. 3.4, the travelling isotropic plate subjected to a constant tension experiences divergence instability at a critical speed

$$
\left(V_{0}^{2}\right)_{*}=\frac{T_{0}}{m}+\frac{\gamma_{*}^{2}}{m} \frac{\pi^{2} D}{\ell^{2}}
$$

where $\gamma_{*}^{2}=\lambda_{*}$ is the minimal eigenvalue of problem (7.1). Parameter $\gamma=\gamma_{*}$ is found as the root of the equation 


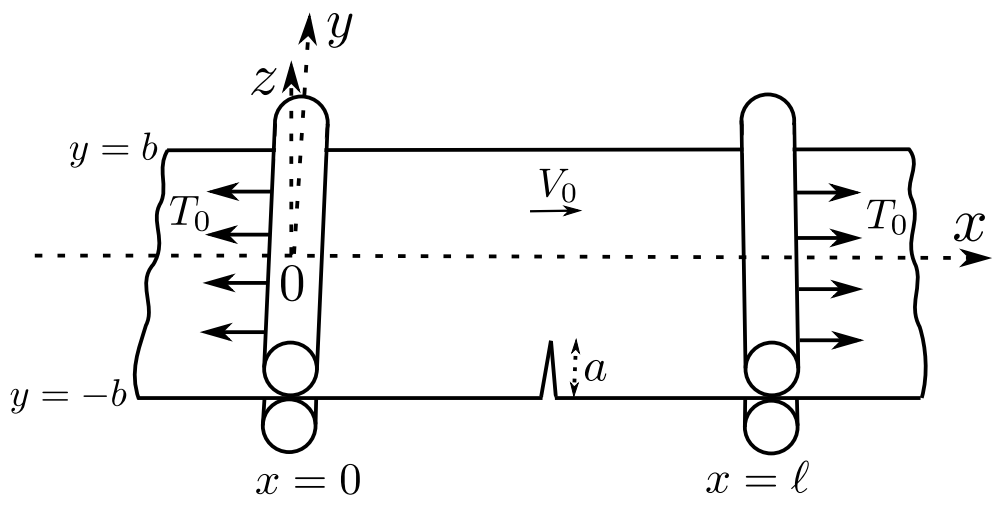

Fig. 7.1 An axially moving elastic plate, containing a small crack at the edge.

$$
\Phi(\gamma, \mu)-\Psi(\gamma, \nu)=0
$$

where

$$
\begin{aligned}
& \Phi(\gamma, \mu)=\tanh \left(\frac{\sqrt{1-\gamma}}{\mu}\right) \operatorname{coth}\left(\frac{\sqrt{1+\gamma}}{\mu}\right) \\
& \Psi(\gamma, \nu)=\frac{\sqrt{1+\gamma}}{\sqrt{1-\gamma}} \frac{(\gamma+\nu-1)^{2}}{(\gamma-\nu+1)^{2}}, \quad \mu=\frac{\ell}{\pi b}
\end{aligned}
$$

For an illustration of the behaviour of $\Phi$ and $\Psi$, see Fig. 3.3 in Sect. 3.4.

As is seen from (7.4-7.5), the root $\gamma=\gamma_{*}$ depends on $\nu$ and $\mu$, and does not depend on the other problem parameters including the value of tension $T_{0}$. Consequently, the critical velocity of instability, defined in (7.3), increases when tension $T_{0}$ is increased. However, $T_{0}$ cannot be increased indefinitely due to initial damages and other imperfections.

Suppose that the plate has small edge cracks that arise at the free boundaries of the plate and have length $a$ with upper bound $a_{*}$, i.e.,

$$
a \leq a_{*} \ll 2 b,
$$

where $a_{*}$ is a given admissible value. The cracks are assumed to be orthogonal to the boundary lines, and thus, the external loading mode is an opening mode and cracks can be considered in the $x y$ plane. If distances between the cracks are large enough, we may consider only one isolated crack of a limited length $a=a_{*}$. This is to say that there is no correlation between the singular stress fields that arise nearby crack ends. We also assume that the rollers do not affect the crack behaviour, i.e., from the viewpoint of a crack, we consider a long travelling plate without supports.

The stress intensity factor $K$ can be expressed as (Westergaard 1939; Irwin 1958)

$$
K=\beta \sigma \sqrt{\pi a} .
$$


Here $\beta=1.12$ is a geometric factor (for $a / 2 b$ small) and $\sigma=\sigma_{x}$ is the axial $x$ component of the stress tensor. In accordance with the brittle fracture condition,

$$
K=K_{a=a_{*}},
$$

and defining fracture toughness $K_{\mathrm{C}}$ such that

$$
K_{\mathrm{C}} \equiv K_{a=a_{*}},
$$

we will have the following expression for the critical value of tension:

$$
\left(T_{0}\right)_{*}=(\sigma h)_{*}=\frac{K_{\mathrm{C}} h}{\beta \sqrt{\pi a_{*}}} .
$$

Thus, safe movement of the plate is realised when

$$
T_{0} \leq \frac{K_{\mathrm{C}} h}{\beta \sqrt{\pi a_{*}}},
$$

and, by (7.3), it is characterised by the following safe range of velocities:

$$
0<V_{0}<\left(V_{0}\right)_{*}=\sqrt{\gamma_{*} \frac{\pi^{2} D}{m \ell^{2}}+\frac{K_{\mathrm{C}} h}{\beta m \sqrt{\pi a_{*}}}} .
$$

If the tension increases, reaching the critical value (7.9) (see Fig. 7.2), then the crack will propagate without stop and cut the plate into two. This process of crack growth occurs in a dynamical manner, and is considered as inadmissible and catastrophic for applications.

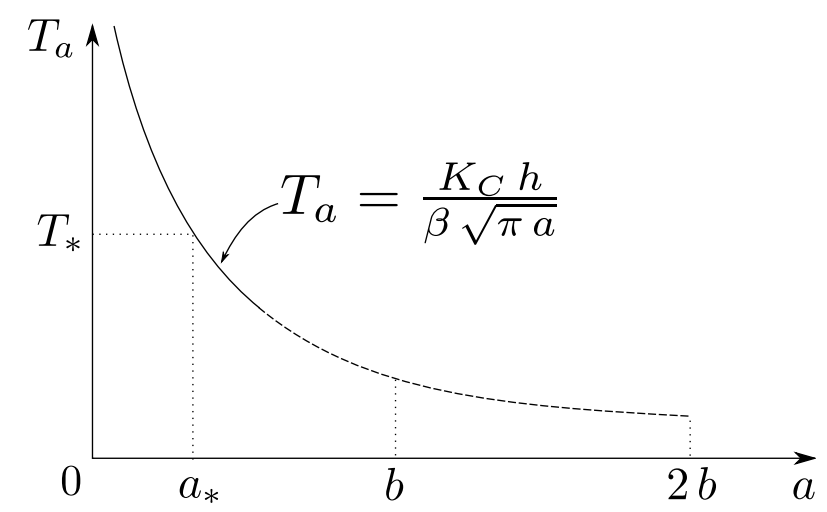

Fig. 7.2 Admissible length of crack $a_{*}$ and critical tension $T_{*}$. A schematic drawing. Note the assumption $a_{*} \ll 2 b$. Hence the displayed equation for $T_{a}$ is only valid (solid line) when $a_{*}$ is small enough. (Reproduced from Banichuk et al 2013) 


\subsubsection{Travelling plates under non-homogeneous tension}

Let us now investigate the case with linearly distributed tension. Again, consider an elastic plate with initial cracks travelling between two rollers at a constant velocity $V_{0}$. Let the plate be subjected to linearly distributed tension

$$
T=T(y)=T_{0}-\alpha y
$$

where $T_{0}>0$ and $\alpha>0$ are given parameters. See Fig. 7.3. For a crack of length $a$, tension at the crack end can now be expressed as

$$
T_{a}=T(a-b)=T_{0}+\alpha(b-a) .
$$

For any given crack length $a$, the corresponding critical tension can be written as

$$
\left(T_{a}\right)_{\mathrm{C}}=\frac{K_{\mathrm{C}} h}{\beta \sqrt{\pi a}}
$$

Suppose that the initial cracks satisfy inequalities (7.6) and that for the most dangerous initial crack equality holds, i.e., $a=a_{*}$. The critical state for the initial crack of length $a=a_{*}$ will be realised, if the tension $T_{a}$ at the crack tip achieves some definite value $T_{*}$, defined by the fracture mechanics condition (7.8). With the critical crack length $a_{*}$, the value of tension at the crack end, (7.13), coincides with the critical value of tension (7.14):

$$
T_{*} \equiv T_{0}+\alpha\left(b-a_{*}\right)=\frac{K_{\mathrm{C}} h}{\beta \sqrt{\pi a_{*}}} .
$$

In the considered case of linearly distributed tension, we introduce some fixed parameter $a_{* *}$ satisfying the conditions

$$
0<a_{*}<a_{* *}<2 b
$$

and consider the interval

$$
0<a \leq a_{* *}
$$

of crack lengths as admissible.

Consider now a crack with length $a$ satisfying (7.17). Depending on the values of the problem parameters, there are two different possible variants of the behaviour of the crack. See Fig. 7.4.

In the first variant, the crack dynamically spreads if $T_{a}>\left(T_{a}\right)_{\mathrm{C}}$, and stops for some crack length in the interval $\left[a_{*}, a_{* *}\right]$, i.e.,

$$
a_{*}<a=a_{\mathrm{st}} \leq a_{* *},
$$

when the following equation holds: 


$$
T_{s t}=T_{0}+\alpha\left(b-a_{\mathrm{st}}\right)=\frac{K_{\mathrm{C}} h}{\beta \sqrt{\pi a_{\mathrm{st}}}} .
$$

In this case, we consider the stopping tension $T_{\text {st }}$ as a critical value of the tension and admit all tensions $T_{0}$ satisfying the condition

$$
0<T_{0} \leq T_{\mathrm{st}}-\alpha\left(b-a_{\mathrm{st}}\right) \equiv\left(T_{0}\right)_{\mathrm{st}}=\left(T_{0}\right)_{\mathrm{cr}}
$$

as a safe tension range.

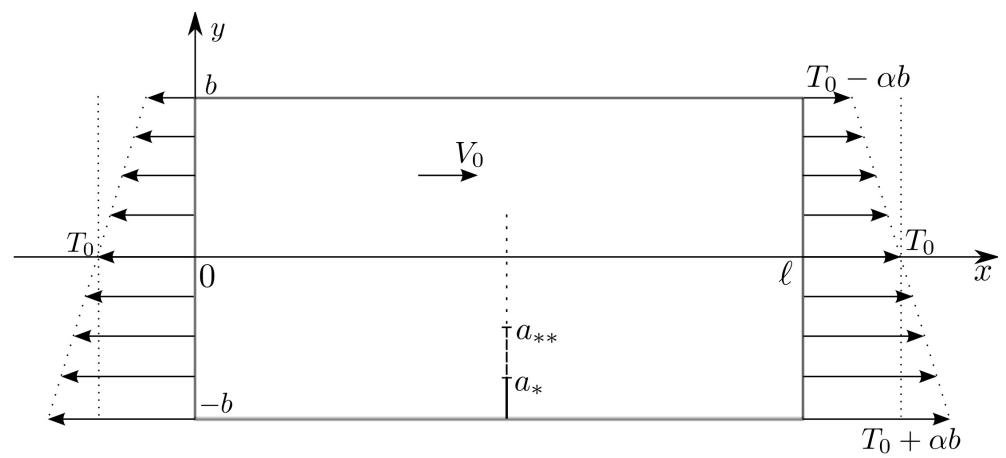

Fig. 7.3 Travelling plate subjected to linearly distributed tension, containing a small crack at the edge.

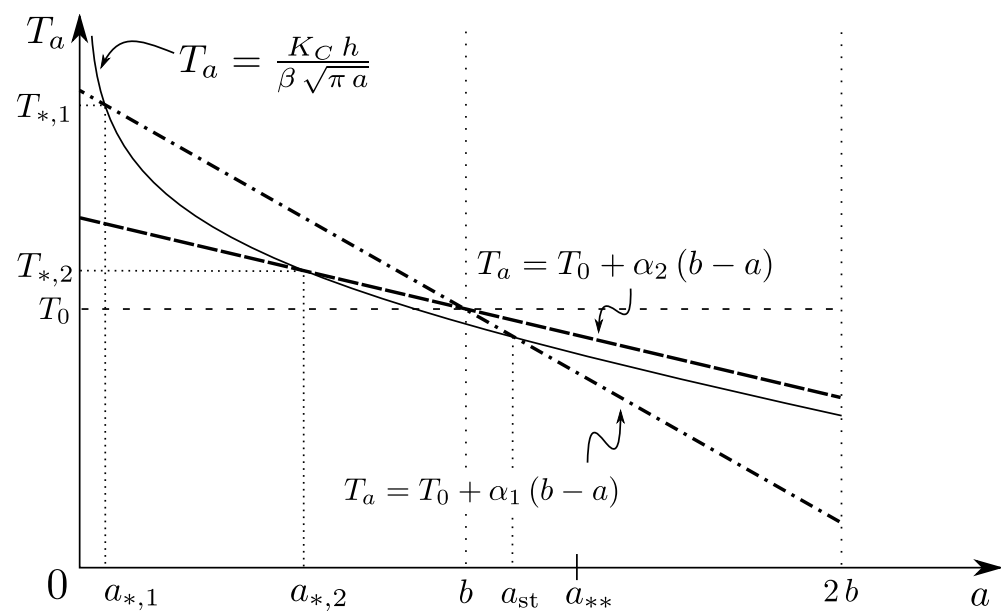

Fig. 7.4 Two variants of tension distributions and critical tension curve. A schematic figure.

In the second variant, when the problem parameters satisfy the inequality 


$$
T_{0}+\alpha\left(b-a_{* *}\right)>\frac{K_{\mathrm{C}} h}{\beta \sqrt{\pi a_{* *}}},
$$

the growth of the crack may stop, when the temporary length $a$ of the crack satisfies the condition

$$
a_{* *}<a=a_{\mathrm{st}}<2 b,
$$

or alternatively, if $a_{\text {st }} \geq 2 b$, the crack will separate the plate into two parts.

Note that the first variant of the crack behaviour discussed above is unexpected in practice, since it requires a relatively steep tension profile, which results in a decrease in the critical transport velocity at the same time (see Chap. 4 and the study Banichuk et al 2010). From the viewpoint of fracture, the case with a linear tension distribution is similar to the case with homogeneous tension. See Fig. 7.4.

\subsection{Cyclic tension and constraints on longevity and instability}

Up to now, we have considered a moving brittle plate with an initial crack, when the in-plane tensions (homogeneous or nonhomogeneous) are fixed. The scenario changes when we study the plate moving under cyclic in-plane tensions and fatigue crack growth.

\subsubsection{Fatigue crack growth and critical conditions}

Suppose that the travelling isotropic plate is subjected to cyclic tension $T$ that varies in the following limits:

$$
\begin{gathered}
T_{\min } \leq T \leq T_{\max }, \\
T_{\min }=T_{0}-\Delta T, \quad T_{\max }=T_{0}+\Delta T,
\end{gathered}
$$

where $\Delta T>0$ is a given parameter such that $T_{0}-\Delta T>0$. See Fig. 7.5.

For one cycle, the tension increases from $T=T_{\min }$ up to $T=T_{\max }$ (the loading process) and then decreases from $T=T_{\max }$ to $T=T_{\min }$ (the unloading process). The loading and unloading processes are assumed quasistatic; dynamical effects are excluded.

Let us apply fatigue crack growth theory, and suppose that the plate contains an initial crack of length $a_{0}$. The process of fatigue crack growth under cyclic tension (loading) can be adequately characterised in the following form (Paris and Erdogan 1963; Forman et al 1967) 


$$
\frac{\mathrm{d} a}{\mathrm{~d} n}=\frac{C(\Delta K)^{k}}{(1-R)\left(K_{\mathrm{C}}-K_{\max }\right)},
$$

where

$$
\begin{gathered}
K_{\max }=\beta \sigma_{\max } \sqrt{\pi a}, \quad K_{\min }=\beta \sigma_{\min } \sqrt{\pi a} \\
\Delta K=K_{\max }-K_{\min }, \quad R=\frac{\sigma_{\min }}{\sigma_{\max }}=\frac{T_{\min }}{T_{\max }}=\frac{K_{\min }}{K_{\max }}, \\
\sigma_{\max }=\frac{T_{\max }}{h}, \quad \sigma_{\min }=\frac{T_{\min }}{h} .
\end{gathered}
$$

Here $C$ and $k$ are material constants, $h$ is the thickness of the plate, $n$ is the number of cycles, and $\sigma_{\max }, K_{\max }, \sigma_{\min }$, and $K_{\min }$ are, respectively, the maximum and minimum values of the stress $\sigma$ and stress intensity factor $K$ in any given loading cycle.

The ordinary differential equation (7.24) defines a quasistatic process of crack growth, and determines the dependence of the crack length $a$ on the number of cycles $n$. This equation is valid up to the moment when $a=a_{*}$, and unstable crack growth (fracture of the plate) is attained.

Suppose that unstable crack growth is attained after $n=n_{*}$ cycles, when the critical crack length $a_{*}$ satisfies the limiting relation

$$
\left(K_{\max }\right)_{a=a_{*}}=K_{\mathrm{C}},
$$

or in another form,

$$
\left(K_{\max }\right)_{a=a_{*}}=\beta \frac{T_{\max }}{h} \sqrt{\pi a_{*}}=K_{\mathrm{C}} .
$$

Note that $\sigma_{\max }$ and $T_{\max }$ (respectively $\sigma_{\min }$ and $T_{\min }$ ) are the maximum (minimum) stresses and tensions in the uncracked plate at the location of the crack. Thus, the structural longevity can be measured by the number of load cycles

$$
n=n_{*},
$$

for which $a=a_{*}$ (realising unstable fracture). In the analysis process, the longevity constraint can be taken as

$$
n_{*} \geq n_{\mathrm{C}},
$$

where $n_{\mathrm{C}}$ is a given minimum value of cycles.

From (7.31), we have

$$
a_{*}=\frac{1}{\pi}\left(\frac{K_{\mathrm{C}} h}{\beta T_{\max }}\right)^{2} .
$$



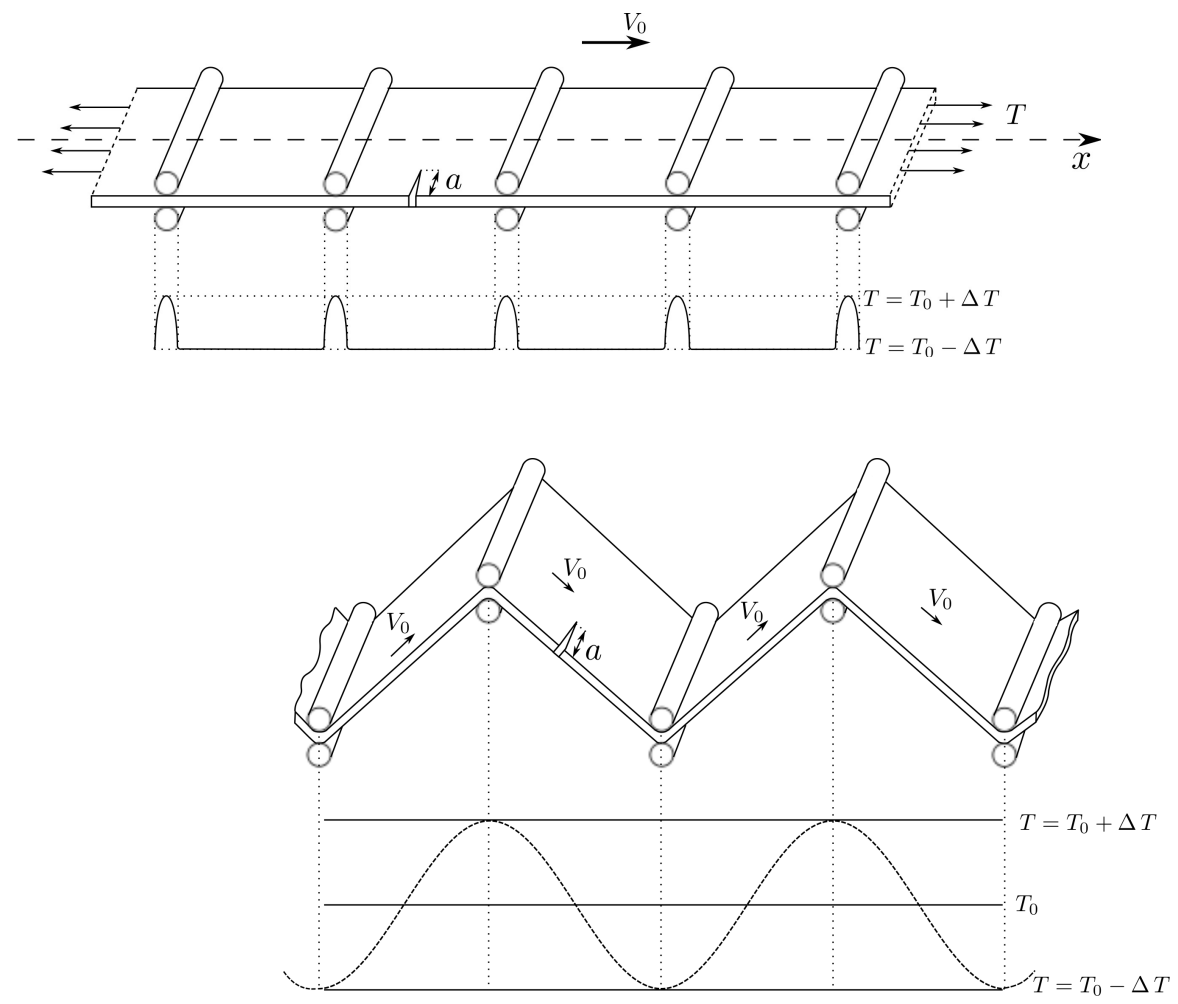

Fig. 7.5 Possible causes for cyclic tension. Top: Tension differences produced by a tension increase in the nip area (see Hristopulos and Uesaka 2002). Bottom: tension differences produced by the Earth's gravity (see Banichuk et al 2011).

Using (7.25-7.29), (7.34), and performing integration in (7.24), we obtain

$$
n_{*}=\frac{1}{B} \int_{a_{0}}^{a_{*}} \frac{\left(\sqrt{a_{*}}-\sqrt{a}\right)}{a^{k / 2}} \mathrm{~d} a=\frac{A}{B},
$$

where

$$
A=\left\{\begin{array}{lr}
2\left[\frac{\sqrt{a_{*}}}{2-k}\left(a_{*}^{(2-k) / 2}-a_{0}^{(2-k) / 2}\right)-\frac{1}{3-k}\left(a_{*}^{(3-k) / 2}-a_{0}^{(3-k) / 2}\right)\right] & \text { if } k \neq 2, k \neq 3, \\
\sqrt{a_{*}}\left(\log a_{*}-\log a_{0}\right)-2\left(a_{*}^{1 / 2}-a_{0}^{1 / 2}\right) & \text { if } k=2, \\
-2\left(1-\sqrt{a_{*}} / \sqrt{a_{0}}\right)-\log a_{*}+\log a_{0} & \text { if } k=3,
\end{array}\right.
$$

and 


$$
B=C\left(\frac{2 \sqrt{\pi} \beta \Delta T}{h}\right)^{k-1}
$$

The critical value of maximum tension $\left(T_{\max }\right)_{*}$ is found, first, by solving $a_{*}$ for a given $n_{*}=n_{\mathrm{C}}$ from (7.35), and then, calculating the corresponding tension from (7.31). The critical value of the average tension $\bar{T}_{*}$ will then be found by (7.23). The safe range of velocities can be estimated as

$$
0<V_{0}<\left(V_{0}\right)_{*}=\sqrt{\gamma_{*} \frac{\pi D}{m \ell^{2}}+\frac{\bar{T}_{*}}{m}},
$$

where $\gamma_{*}$ is the root of $(7.4)$.

\subsubsection{Numerical examples}

In the following, the analytical results obtained for some critical crack lengths, critical tensions and critical transport velocities will be illustrated for parameter values describing paper material. The used material constants and geometric constants are presented in Table 7.1. The fracture toughness $K_{\mathrm{C}}$ of paper is calculated from the fundamental relation $G_{\mathrm{C}}=K_{\mathrm{C}}^{2} / E$ (see e.g. Irwin 1958), since there are measured values for the strain energy release rate $G_{\mathrm{C}}$.

\begin{tabular}{|c|c|c|c|c|c|}
\hline \multicolumn{6}{|c|}{ Material constants } \\
\hline $\bar{E}$ & $\nu$ & $m$ & $G_{\mathrm{C}} / \rho($ Seth and Page 1974$)$ & $k$ & $C$ \\
\hline \multirow[t]{4}{*}{$10^{9} \mathrm{~N} / \mathrm{m}^{2}$} & 0.3 & $0.08 \mathrm{~kg} / \mathrm{m}^{2}$ & $10 \mathrm{Jm} / \mathrm{kg}$ & 3 & $10^{-14}$ \\
\hline & \multicolumn{3}{|c|}{ Geometric constants } & & \\
\hline & $\bar{\ell}$ & $b($ plat & half-width) $h$ & & \\
\hline & & $0.5 \mathrm{~m}$ & $10^{-4} \mathrm{~m}$ & & \\
\hline
\end{tabular}

Table 7.1 Physical parameters used in the numerical examples.

In Fig. 7.6, top, the predicted critical tension $\left(T_{0}\right)_{*}$ is plotted with respect to the critical crack length $a_{*}$, as per (7.9). Two different values of $G_{\mathrm{C}} / \rho$ are illustrated: the smaller one (10 Jm/ kg) corresponding to newsprint and the larger one $(20 \mathrm{Jm} / \mathrm{kg}$ ) corresponding to writing paper.

Note that we assume $a_{*} \ll 2 b$, which allows us to use a constant crack geometry factor $\beta=1.12$. The results in Fig. 7.6, although plotted for the range $0 \leq a_{*} \leq 1 \mathrm{~m}$, may not be valid for $a_{*}$ close to $1 \mathrm{~m}$, if $2 b=1 \mathrm{~m}$. However, the results in Fig. 7.6 are independent from the parameter $b$. Only 
the accuracy of the results depends on $b$. Thus, the results for $a_{*}$ close to $1 \mathrm{~m}$ are valid for very wide plates, when $2 b \gg 1 \mathrm{~m}$.

On the bottom of Fig. 7.6, the critical velocity $\left(V_{0}\right)_{*}$ is plotted with respect to the critical crack length $a_{*},(7.11)$. The results suggest that higher safe plate speeds are achieved for plates with smaller initial cracks.
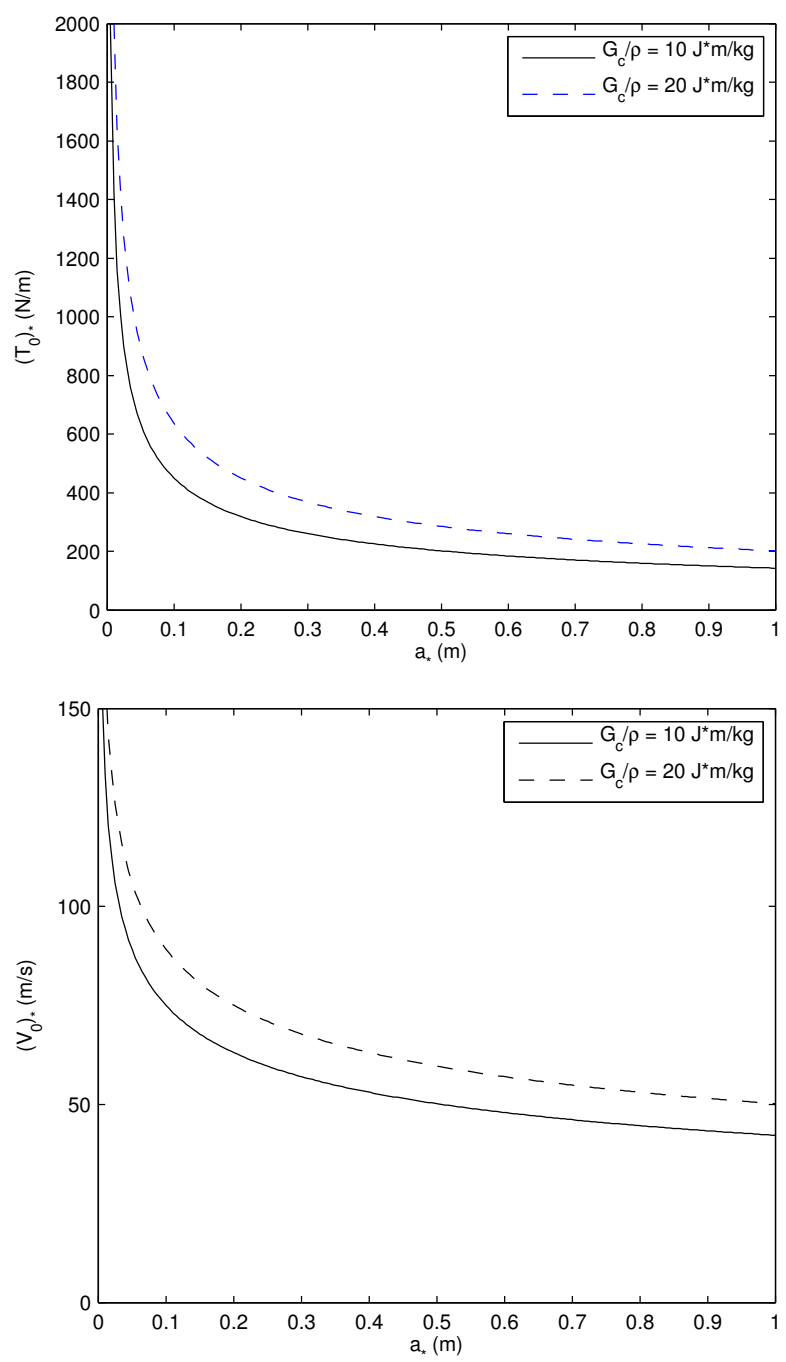

Fig. 7.6 Top: Critical tension and; Bottom: the corresponding critical velocity with respect to the critical crack length for two different values of the strain energy rate. The value $G_{\mathrm{C}} / \rho=10 \mathrm{Jm} / \mathrm{kg}$ corresponds to newsprint and $20 \mathrm{Jm} / \mathrm{kg}$ to writing paper. Note the assumption $a_{*} \ll 2 b$ made above. 

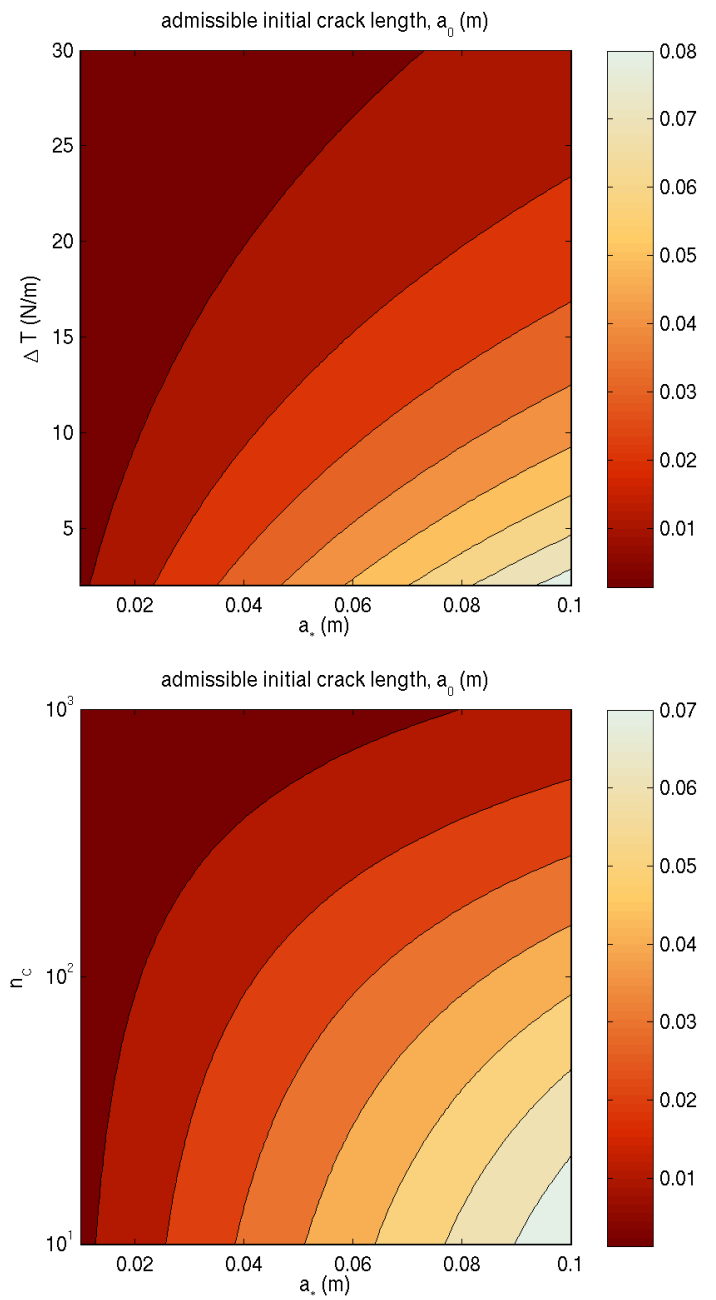

Fig. 7.7 Admissible initial crack length $a_{0}$ with respect to the critical crack length $a_{*}$ and the variance in tension $\Delta T$ (left) or the critical number of cycles $n_{\mathrm{C}}$ (right). Note the roles of $a_{0}$ and $a_{*}$. Top: The critical number of cycles is constant, $n_{\mathrm{C}}=100$. Bottom: The variance in tension is constant, $\Delta T=10 \mathrm{~N} / \mathrm{m}$. Note the logarithmic scale of $n_{\mathrm{C}}$.

In Figs. $7.7-7.9$, the results of the longevity analysis are shown. The used strain energy rate over density was $G_{\mathrm{C}} / \rho=10 \mathrm{Jm} / \mathrm{kg}$ (newsprint).

In Fig. 7.7, the admissible critical crack length is considered known, and the corresponding admissible initial crack length is found. The admissible initial crack length $a_{0}$ is presented as a coloured sheet with respect to the critical crack length $a_{*}$ and the variance in the tension $\Delta T$ (top) or the critical number of cycles $n_{\mathrm{C}}$ (bottom). The critical crack length $a_{*}$ is given values in 


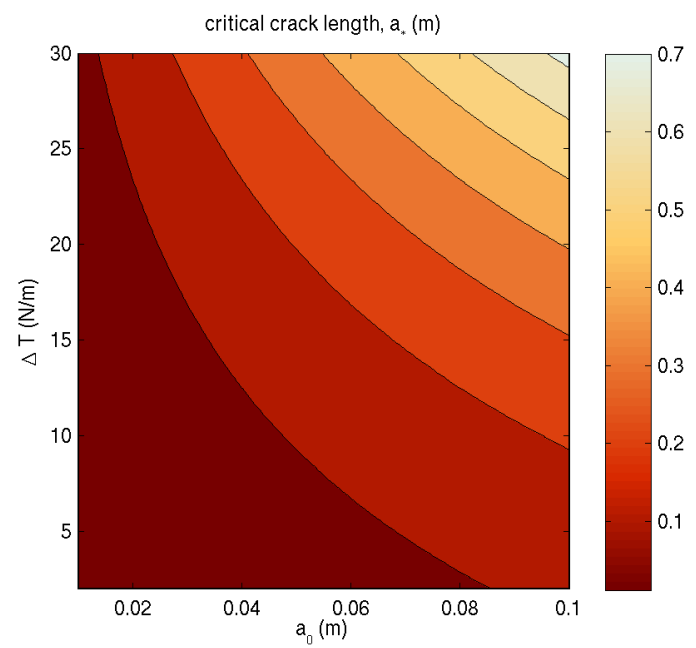

Fig. 7.8 Critical crack length $a_{*}$ with respect to the initial crack length $a_{0}$ and the variance in tension $\Delta T$. The critical number of cycles is constant, $n_{\mathrm{C}}=100$. Note the roles of $a_{0}$ and $a_{*}$.

the range $[0.01,0.1] \mathrm{m}$, the variance in tension in the range $[2,30] \mathrm{N} / \mathrm{m}$, and the critical number of cycles is in the range [10, 1000]. In Fig. 7.7 on the top, the number of cycles is kept constant, $n_{\mathrm{C}}=n_{*}=100$. On the right hand side, the variance in tension is constant, $\Delta T=10 \mathrm{~N} / \mathrm{m}$.

With the chosen problem parameters, increasing the number of cycles only slightly decreases the (admissible) initial crack length. The more cycles we require, the smaller the initial cracks that can be accepted. The effect of the length of the critical crack is shown clearly, and as expected, the smaller the critical crack length the smaller is the initial crack that can be accepted (Fig. 7.6, bottom).

In Figs. $7.8-7.9$, the initial crack length $a_{0}$ is known, and the corresponding critical crack length $a_{*}$ is predicted. In these figures, the critical crack length $a_{*}$, the corresponding critical tension $\bar{T}_{*}$, and the critical velocity $\left(V_{0}\right)_{*}$ (estimate) are presented as a coloured sheet with respect to the initial crack length $a_{0}$ and the variance in the tension $\Delta T$. The initial crack length $a_{0}$ is given values in the range $[0.01,0.1] \mathrm{m}$, and the variance in tension is in the range $[2,30] \mathrm{N} / \mathrm{m}$. The critical number of cycles is constant, $n_{\mathrm{C}}=n_{*}=100$. As we can see from the figures, the results are as expected: if the variance in tension is large, only minimal initial cracks can be accepted.

It should be pointed out that in the figures, the critical conditions are shown. Also the number of cycles corresponds to the critical situation. Furthermore, these results are mainly qualitative. Hence, additional safety margins may be required in practice to avoid web breaks. 

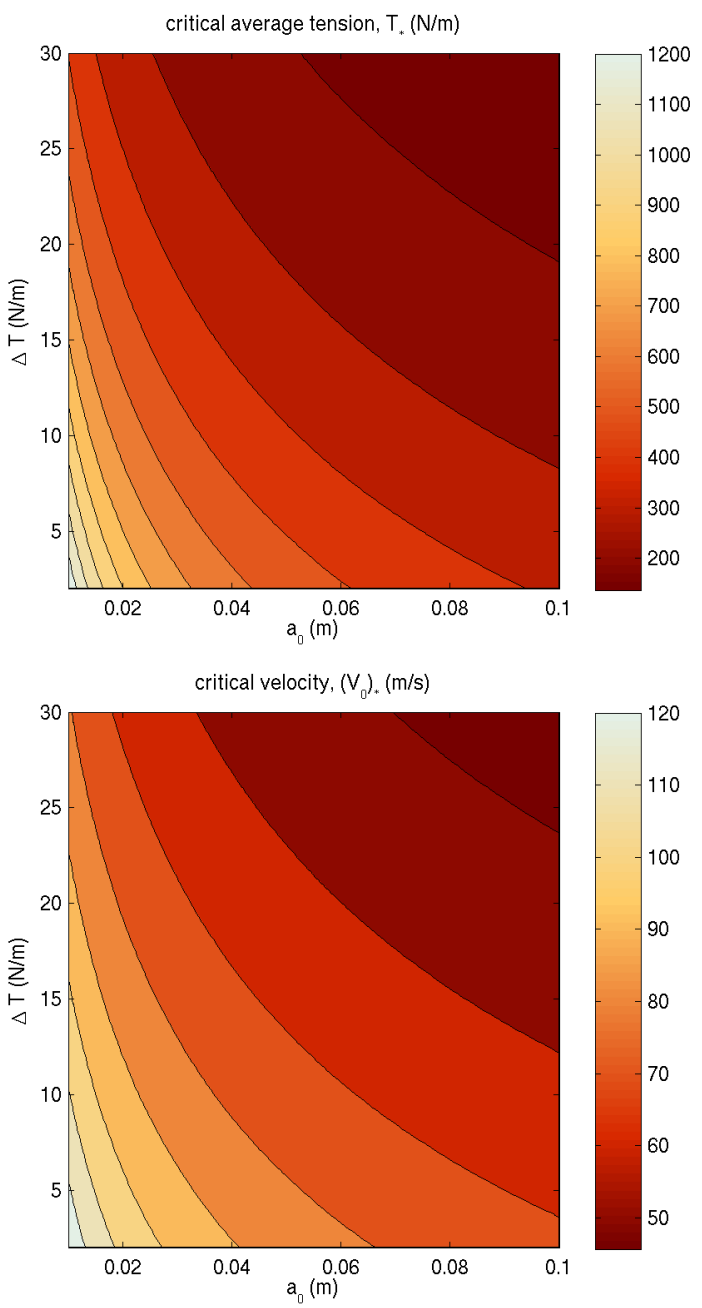

Fig. 7.9 Top: Critical average tension $T_{*}$ and; Bottom: transport velocity $\left(V_{0}\right)_{*}$ with respect to the initial crack length $a_{0}$ and the variance in tension $\Delta T$. The critical number of cycles is constant, $n_{\mathrm{C}}=100$. 


\section{References}

Banichuk N, Jeronen J, Neittaanmäki P, Tuovinen T, Saksa T (2010) Theoretical study on travelling web dynamics and instability under a linear tension distribution. Tech. Rep. B1/2010, University of Jyväskylä, Reports of the Department of Mathematical Information Technology. B: Scientific Computing.

Banichuk N, Jeronen J, Saksa T, Tuovinen T (2011) Static instability analysis of an elastic band travelling in the gravitational field. Rakenteiden mekaniikka (Journal of Structural Mechanics) 44(3):172-185

Banichuk N, Kurki M, Neittaanmäki P, Saksa T, Tirronen M, Tuovinen $\mathrm{T}$ (2013) Optimization and analysis of processes with moving materials subjected to fatigue fracture and instability. Mechanics Based Design of Structures and Machines: An International Journal 41(2):146-167, URL http://dx.doi.org/10.1080/15397734.2012.708630

Forman RG, Kearney VE, Engle RM (1967) Numerical analysis of crack propagation in cyclic-loaded structure. Journal of Basic Engineering, Transactions of the American Society of Mechanical Engineers D 89:459-464

Hristopulos DT, Uesaka T (2002) A model of machine-direction tension variations in paper webs with runnability applications. Journal of Pulp and Paper Science 28(12):389-394

Irwin GR (1958) Fracture. In: Flugge S (ed) Handbuch der Physik, vol VI, Springer-Verlag, Berlin, pp 551-590

Paris PC, Erdogan F (1963) A critical analysis of crack propagation laws. Journal of Basic Engineering, Transactions of the American Society of Mechanical Engineers D 85:528-534

Seth RS, Page DH (1974) Fracture resistance of paper. Journal of Materials Science 9(11):1745-1753, URL http://dx.doi.org/10.1007/BF00541741

Westergaard HM (1939) Bearing pressures and cracks. Journal of Applied Mechanics 6:A49-A53 



\title{
Chapter 8 Some optimization problems ${ }^{1}$
}

\begin{abstract}
In this chapter, the problems of safety analysis and optimization of a moving elastic plate travelling between two rollers at a constant axial velocity are considered. We will use a model of a thin elastic plate subjected to bending and in-plane tension (distributed membrane forces). We will study transverse buckling (divergence) of the plate and its brittle and fatigue fracture caused by fatigue crack growth under cyclic in-plane tension (loading). Our aim is to find the safe ranges of velocities of an axially moving plate analytically under the constraints of longevity and stability.

In the end of this chapter, the expressions for critical buckling velocity and the number of cycles before the fracture (longevity of the plate) as a function of in-plane tension and other problem parameters are used for formulation and we will study the case as an optimization problem. Our target is to find the optimal in-plane tension to maximize the performance function of paper production. This problem is solved analytically and the obtained results are presented as formulae and numerical tables.
\end{abstract}

\subsection{Optimization of moving plates subjected to instability and fracture}

It is known that, in systems with travelling continuum, an increase in tension has a stabilizing effect but a decrease in tension may lead to a loss of stability. From the viewpoint of fracture, tension has an opposite effect: high tension may lead to growing or arising of cracks, and tension low enough then guarantees safe conditions. In practice, both instability and material fracture may lead to web breaks.

1 Co-author in this chapter: Maria Tirronen, Department of Mathematical Information Technology, University of Jyväskylä 

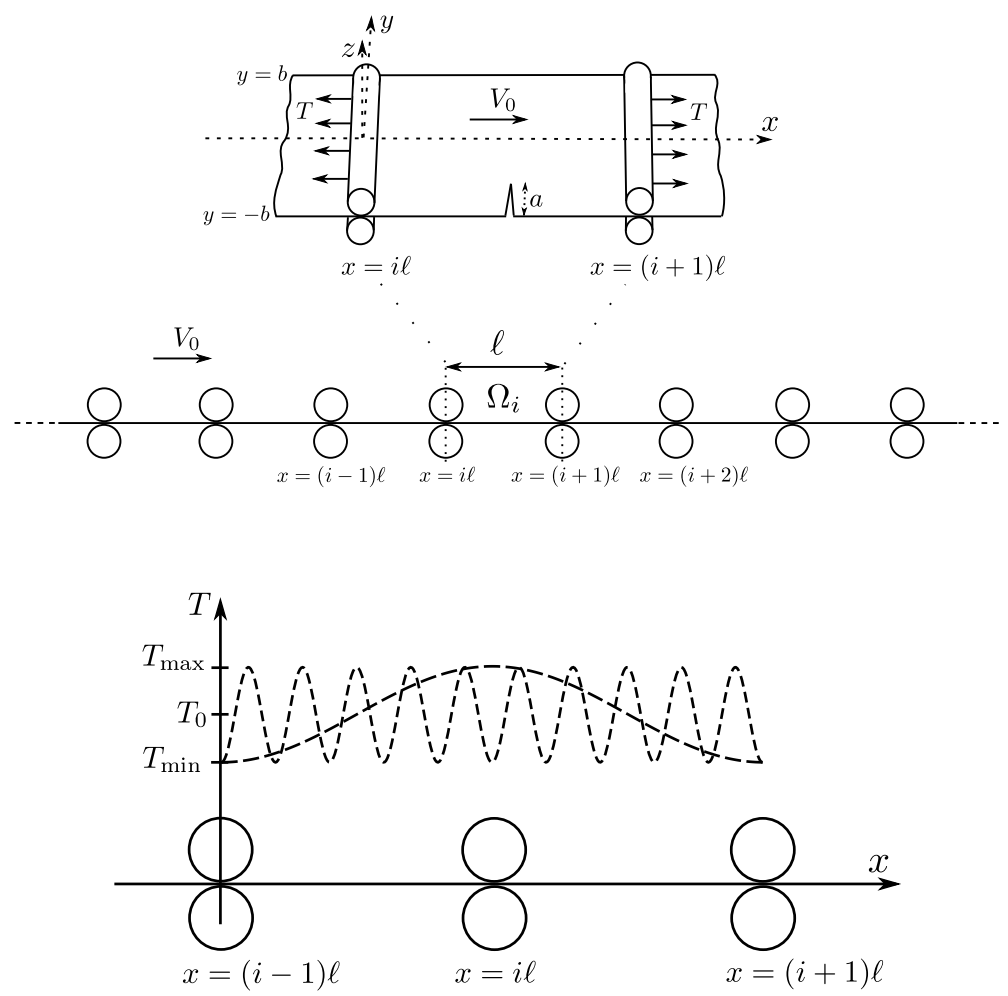

Fig. 8.1 Top: A plate travelling through a system of supporting rollers, and having an initial crack. Bottom: Examples of cyclic tension. There may be few or many tension cycles per span. (Reproduced from Banichuk et al 2013)

In this section, we will present constraints for the plate velocity and the structural longevity so that the considered system would perform in a safe manner. By longevity or structural longevity, we refer to the number of cycles that the (cracked) material sustains before fracture failure. We will also construct a productivity function, with the help of which an optimal value for in-plane tension will be sought.

\subsubsection{Optimization criterion and constraints}

Consider an elastic plate travelling at a constant velocity $V_{0}$ in the $x$ direction and being simply supported by a system of rollers located at $x=0, \ell, 2 \ell, 3 \ell, \ldots$ (Fig. 8.1). A rectangular element $\Omega_{i}, i=0,1,2, \ldots$, of the plate 


$$
\Omega_{i} \equiv\left\{(x, y) \in \mathbb{R}^{2} \mid i \ell<x<(i+1) \ell,-b<y<b\right\}
$$

is considered in a cartesian coordinate system, where $\ell$ and $b$ are prescribed geometric parameters. Additionally, assume that the considered plate is represented as an isotropic elastic plate having constant thickness $h$, Poisson ratio $\nu$, Young modulus $E$, and bending rigidity $D$. The plate elements in (8.1) have small initial cracks (Fig. 8.1) of length $a$ with a given upper bound $a_{0}$, i.e.,

$$
0<a \leq a_{0},
$$

and are subjected to homogeneous tension $T$, acting in the $x$ direction.

The sides of the plate element $(i=1,2,3, \ldots)$

$$
\{x=i \ell, \quad-b \leq y \leq b\} \quad \text { and } \quad\{x=(i+1) \ell, \quad-b \leq y \leq b\}
$$

are simply supported and the sides

$$
\{y=-b, \quad i \ell \leq x \leq(i+1) \ell\} \quad \text { and } \quad\{y=b, \quad i \ell \leq x \leq(i+1) \ell\}
$$

are free of tractions.

Consider the following scenario, where the plate is moving under cyclic in-plane tension and fatigue crack growth is realised. Suppose that the plate is subjected to a cyclic tension $T$ that varies in the given limits

$$
T_{\min } \leq T \leq T_{\max }
$$

where

$$
T_{\min }=T_{0}-\Delta T, \quad T_{\max }=T_{0}+\Delta T .
$$

Above, $\Delta T>0$ is a given parameter such that

$$
T_{0}-\Delta T>0 \text { and } \frac{\Delta T}{T_{0}} \ll 1 .
$$

For one cycle, the tension increases from $T=T_{\min }$ up to $T=T_{\max }$ (the loading process) and then decreases from $T=T_{\max }$ to $T=T_{\min }$ (the unloading process). The loading and unloading processes are supposed to be quasistatic; dynamical effects are excluded.

The product of the plate velocity $V_{0}$ and the process time $t_{\mathrm{f}}$ can be considered a productivity criterion (performance function), i.e.,

$$
J=m_{0} V_{0} t_{\mathrm{f}}, \quad m_{0}=2 b m .
$$

Here, $m$ is the mass per unit area of the plate. In (8.4), the velocity $V_{0}$ is taken from the safe interval

$$
0<V_{0}<V_{0}^{\text {cr }}
$$


where $V_{0}^{\text {cr }}$ is the critical buckling speed.

A safe interval for the safe functioning time (number of cycles) is written as

$$
0<t_{\mathrm{f}}<t_{\mathrm{f}}^{\text {cr }} \quad \text { or } \quad 0<n<n^{\text {cr }},
$$

where $t_{\mathrm{f}}^{\text {cr }}$ and $n^{\text {cr }}$ are, respectively, the time interval and the total number of cycles before fatigue fracture. For a small cycle time period $\tau$ and a big number of cycles $n$, we assume that $t_{\mathrm{f}} \approx n \tau$. Note that the critical buckling velocity $V_{0}^{\text {cr }}$ and the critical functioning time $t_{\mathrm{f}}^{\text {cr }}$ (critical number of cycles $n^{\text {cr }}$ ) depend on the parameters of the average in-plane tension $T_{0}$, and the admissible variance $\Delta T$, i.e

$$
\begin{gathered}
V_{0}^{\mathrm{cr}}=V_{0}^{\mathrm{cr}}\left(T_{0}, \Delta T\right), \\
t_{\mathrm{f}}^{\mathrm{cr}}=t_{\mathrm{f}}^{\mathrm{cr}}\left(T_{0}, \Delta T\right), \\
n^{\mathrm{cr}}=n^{\mathrm{cr}}\left(T_{0}, \Delta T\right) .
\end{gathered}
$$

Consequently, the maximum value of the productivity criterion for the given values $T_{0}$ and $\Delta T$ is evaluated as

$$
\begin{aligned}
J\left(T_{0}, \Delta T\right) & =m_{0} V_{0}^{\mathrm{cr}}\left(T_{0}, \Delta T\right) t_{\mathrm{f}}^{\mathrm{cr}}\left(T_{0}, \Delta T\right) \\
& =m_{0} \tau V_{0}^{\mathrm{cr}}\left(T_{0}, \Delta T\right) n^{\mathrm{cr}}\left(T_{0}, \Delta T\right) .
\end{aligned}
$$

The optimal average (mean) in-plane tension $T_{0}$ is found by solving the following optimization problem:

$$
J^{*}=\max _{T_{0}} J\left(T_{0}, \Delta T\right) .
$$

To solve the formulated optimization problem, we will use the explicit analytical expressions for the values $V_{0}^{\text {cr }}$ and $n^{\text {cr }}$. The value of $T_{0}$, giving the maximal production $J^{*}$, is denoted by $T_{0}^{*}$.

To evaluate $n^{\text {cr }}$, let us apply fatigue crack growth theory. Suppose that the plate contains one initial crack of length $a_{0}$. The process of fatigue crack growth under a cyclic tension (loading) can be described by the following equation (Paris and Erdogan 1963) and initial condition

$$
\frac{\mathrm{d} a}{\mathrm{~d} n}=C \kappa_{0}^{k} a^{k / 2},
$$

where

$$
\kappa_{0}=\frac{2 \beta \sqrt{\pi}}{h} \Delta T .
$$


The variance $\Delta K$ of the stress intensity factor $K$ is determined with the help of the formulae

$$
\begin{gathered}
\Delta K=K_{\max }-K_{\min }, \quad K_{\max }=\beta \sigma_{\max } \sqrt{\pi a}, \\
K_{\min }=\beta \sigma_{\min } \sqrt{\pi a}, \quad \sigma_{\max }=\frac{T_{\max }}{h}, \quad \sigma_{\min }=\frac{T_{\min }}{h} .
\end{gathered}
$$

In (8.5), $C$ and $k$ are material constants. In (8.6), $h$ is the thickness of the plate, $n$ is the number of cycles, and $\sigma_{\max }, K_{\max }, \sigma_{\min }$ and $K_{\min }$ are, respectively, the maximum and minimum values of the stress $\sigma$ and the stress intensity factor $K$ in any given loading cycle. For the considered case, the surface crack geometric factor is $\beta=1.12$.

It follows from (8.5) and that for considered values of the parameter $k \neq 2$, we will have

$$
n=A\left[\frac{1}{a_{0}^{(k-2) / 2}}-\frac{1}{a^{(k-2) / 2}}\right],
$$

where

$$
A=\frac{2}{(k-2) C \kappa_{0}^{k}} .
$$

Unstable crack growth is obtained after $n=n^{\text {cr }}$ cycles when the critical crack length $a_{\text {cr }}$ satisfies the limiting relation

$$
\left(K_{\max }\right)_{a=a_{\mathrm{cr}}}=K_{\mathrm{C}}
$$

or, in another form, we have

$$
\beta \frac{T_{\max }}{h} \sqrt{\pi a_{\mathrm{cr}}}=K_{\mathrm{C}} .
$$

The quantities $\sigma_{\max }$ and $T_{\max }$ (respectively $\sigma_{\min }$ and $T_{\min }$ ) are the maximum (minimum) stresses and tensions in the uncracked plate, where the crack is located. Using (8.8) and the inequality $\Delta T / T_{0} \ll 1$, we obtain

$$
a_{\mathrm{cr}}=\frac{1}{\pi}\left(\frac{K_{\mathrm{C}} h}{\beta T_{\max }}\right)^{2} \approx \frac{1}{\pi}\left(\frac{K_{\mathrm{C}} h}{\beta T_{0}}\right)^{2}
$$

and, consequently, we will have the following expression for the critical number of cycles:

$$
n^{\mathrm{cr}}=(n)_{a=a_{\mathrm{cr}}}=A\left[\frac{1}{a_{0}^{(k-2) / 2}}-\left(\frac{\sqrt{\pi} \beta T_{0}}{K_{\mathrm{C}} h}\right)^{k-2}\right] .
$$

From the condition of positiveness of the expression in (8.9), we find the maximum value of admissible tensions, 


$$
T_{0} \leq \frac{1}{\sqrt{\pi a_{0}}} \frac{K_{\mathrm{C}} h}{\beta} \equiv T_{0}^{\mathrm{M}} .
$$

In the special case $k=2$, we can find the critical number of cycles to be

$$
n^{\mathrm{cr}}=B \ln \left[\frac{1}{\pi a_{0}}\left(\frac{K_{\mathrm{C}} h}{\beta T_{0}}\right)^{2}\right]
$$

where

$$
B=\frac{1}{C \kappa_{0}^{2}},
$$

and the tension limit $T_{0}^{\mathrm{M}}$ is expressed by (8.10).

The dependence of the critical number of cycles $n^{\mathrm{cr}}$ on the average tension $T_{0}$ and the problem parameter $k$ is shown in Fig. 8.2 using dimensionless quantities that will be presented below in (8.19) and (8.24).

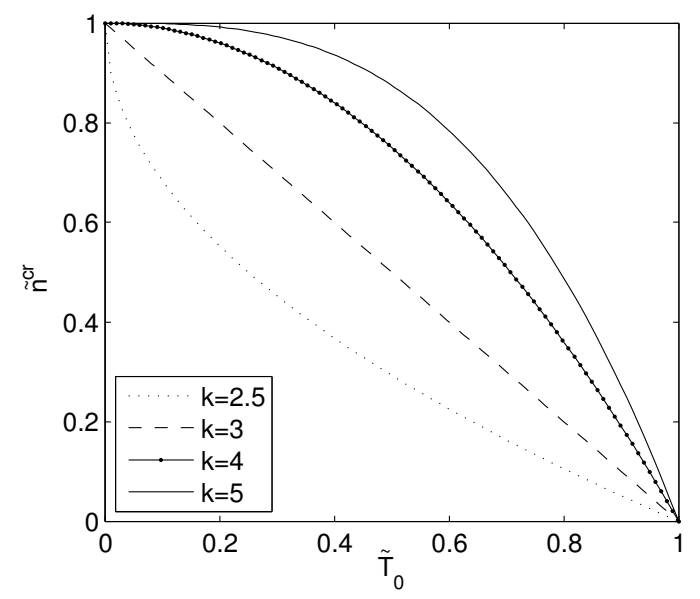

Fig. 8.2 Dependence of the normalized critical number of cycles, (8.25), on the dimensionless average tension, (8.20).

The critical velocity of static instability (buckling) of the travelling plate, as was discussed in Sect. 3.4 (see also Banichuk et al 2010), is given by the following formula:

$$
\left(V_{0}^{\mathrm{cr}}\right)^{2}=\frac{T_{0}}{m}+\frac{\gamma_{*}^{2}}{m} \frac{\pi^{2} D}{\ell^{2}}
$$

where $D=E h^{3} /\left[12\left(1-\nu^{2}\right)\right], m$ is the mass per unit area (of the plate), and $\gamma=\gamma_{*}$ is the root of the equation (see Fig. 3.3)

$$
\Phi(\gamma, \mu)-\Psi(\gamma, \nu)=0
$$


where

$$
\begin{gathered}
\Phi(\gamma, \mu)=\tanh \left(\frac{\sqrt{1-\gamma}}{\mu}\right) \operatorname{coth}\left(\frac{\sqrt{1+\gamma}}{\mu}\right), \\
\Psi(\gamma, \nu)=\frac{\sqrt{1+\gamma}}{\sqrt{1-\gamma}} \frac{(\gamma+\nu-1)^{2}}{(\gamma-\nu+1)^{2}}, \quad \mu=\frac{\ell}{\pi b} .
\end{gathered}
$$

As is seen from (8.13) and (8.14), the root $\gamma=\gamma_{*}$ depends on $\nu$ and $\mu$, and does not depend on the other problem parameters including the value of tension $T_{0}$. Consequently, the critical instability velocity, defined in (8.12), increases when tension $T_{0}$ is increased. However, $T_{0}$ cannot be increased indefinitely due to initial damages and other imperfections.

\subsubsection{Finding optimal solution}

The most important factor, for the runnability and stability of moving plates containing initial imperfections, is the applied tension. To find a safe and optimal $T_{0}$ maximizing the performance function is the problem we will consider in this section.

Let us represent the functional to be optimized, (8.4), as a function of the average tension $T_{0}$. By taking into account explicit expressions for $n^{\mathrm{cr}}$, in (8.9), and for $V_{0}^{\mathrm{cr}}$, in (8.12), and performing necessary algebraic transformations, assuming that $k \neq 2$, we will have

$$
\begin{gathered}
J\left(T_{0}\right)=m_{0} \tau V_{0}^{\mathrm{cr}}\left(T_{0}\right) n^{\mathrm{cr}}\left(T_{0}\right) \\
=J_{0}\left[1+\frac{1}{D}\left(\frac{\ell}{\gamma_{*} \pi}\right)^{2} T_{0}\right]^{1 / 2}\left[1-\left(\frac{\beta \sqrt{\pi a_{0}}}{h K_{\mathrm{C}}} T_{0}\right)^{k-2}\right]
\end{gathered}
$$

where

$$
J_{0}=m_{0} \tau C_{V_{0}} C_{n},
$$

and

$$
C_{V_{0}}=\frac{\pi \gamma_{*} \sqrt{D}}{\ell \sqrt{m}}
$$

and

$$
C_{n}=\frac{2 a_{0}}{(k-2) C}\left(\frac{h}{2 \beta \Delta T \sqrt{\pi a_{0}}}\right)^{k} .
$$

The performance function $J$ is proportional to the multiplier $J_{0}$, and consequently, the optimized tension $T_{0}$ does not depend on $J_{0}$.

For convenience of the following estimations and reduction of characteristic parameters, we introduce the dimensionless values 


$$
\begin{gathered}
\tilde{J}=\frac{J}{J_{0}}, \\
\tilde{T}_{0}=\frac{T_{0}}{T_{0}^{\mathrm{M}}}=\frac{\beta \sqrt{\pi a_{0}}}{K_{\mathrm{C}} h} T_{0}, \\
g=\frac{K_{\mathrm{C}} h}{\beta D \sqrt{\pi a_{0}}}\left(\frac{\ell}{\gamma_{*} \pi}\right)^{2} .
\end{gathered}
$$

The optimized functional and the interval of optimization, in the case when $k>2$, are

$$
\tilde{J}\left(\tilde{T}_{0}\right)=\left(1+g \tilde{T}_{0}\right)^{1 / 2}\left(1-\tilde{T}_{0}^{k-2}\right)
$$

with

$$
0 \leq \tilde{T}_{0} \leq 1
$$

In other words, we consider

$$
\tilde{J}\left(\tilde{T}_{0}\right)=\tilde{V}_{0}^{\mathrm{cr}}\left(\tilde{T}_{0}\right) \tilde{n}^{\mathrm{cr}}\left(\tilde{T}_{0}\right)
$$

with

$$
\tilde{V}_{0}^{\mathrm{cr}}\left(\tilde{T}_{0}\right)=\left(1+g \tilde{T}_{0}\right)^{1 / 2}
$$

and

$$
\tilde{n}^{\operatorname{cr}}\left(\tilde{T}_{0}\right)=1-\tilde{T}_{0}^{k-2} .
$$

In the special case $k=2$, we will use the expressions (8.4), (8.11) and (8.12), and perform algebraic transformations. We will have

$$
\begin{gathered}
J\left(T_{0}\right)=m_{0} \tau V_{0}^{\mathrm{cr}}\left(T_{0}\right) n^{\mathrm{cr}}\left(T_{0}\right)= \\
J_{1}\left[1+\frac{1}{D}\left(\frac{\ell}{\gamma_{*} \pi}\right)^{2} T_{0}\right]^{1 / 2} \ln \left(\frac{h K_{\mathrm{C}}}{\beta \sqrt{\pi a_{0}}} \frac{1}{T_{0}}\right)
\end{gathered}
$$

with

$$
J_{1}=\frac{2 m_{0} \tau \pi \gamma_{*} \sqrt{D}}{C \ell \sqrt{m}}\left(\frac{h}{2 \beta \Delta T \sqrt{\pi}}\right)^{2} .
$$

Using the dimensionless values $\tilde{J}=J / J_{1}$ and $\tilde{T}_{0}, g$ from (8.19-8.21), we find that

$$
\tilde{J}\left(\tilde{T}_{0}\right)=\ln \left(\frac{1}{\tilde{T}_{0}}\right)\left(1+g \tilde{T}_{0}\right)^{1 / 2}, \quad 0 \leq \tilde{T}_{0} \leq 1 .
$$

It is seen from (8.26) that

$$
0=(\tilde{J})_{\tilde{T}_{0}=1} \leq \tilde{J}\left(\tilde{T}_{0}\right) \leq \lim _{\tilde{T}_{0} \rightarrow 0}(\tilde{J})=\infty, \quad 0 \leq \tilde{T}_{0} \leq 1
$$

Note that (8.27) also holds in the case $k<2$, when 


$$
\tilde{J}\left(\tilde{T}_{0}\right)=-\left(1+g \tilde{T}_{0}\right)^{1 / 2}\left(1-\tilde{T}_{0}^{k-2}\right)
$$

and

$$
J_{0}=\frac{2 m_{0} \tau \pi a_{0} \gamma_{*} \sqrt{D}}{(2-k) C \ell \sqrt{m}}\left(\frac{h}{2 \beta \Delta T \sqrt{\pi a_{0}}}\right)^{k} .
$$

Thus, in the case $k \leq 2$, the optimum is $\tilde{T}_{0}=0$, which is not a physically meaningful case, since it corresponds to an extremely low plate velocity. However, for most materials $k \approx 3$ or bigger.

\subsubsection{Dependence of optimal solution on problem parameters}

In the following, we will look at some numerical examples. The optimization problem (8.22-8.23) is solved numerically for different values of $k$ : for $k=$ $2.5, k=3$ and $k=3.5$. The material parameters are chosen to describe a paper material. The parameter values used in the examples are given in Table (8.1). Paper fracture toughness $K_{\mathrm{C}}$ is calculated from the fundamental relation $G_{\mathrm{C}}=K_{\mathrm{C}}^{2} / E$. The variance in tension is chosen to be small, $\Delta T=$ $0.1 \mathrm{~N} / \mathrm{m}$. The values of initial crack lengths used in the examples are $a_{0}=$ $0.005 \mathrm{~m}, 0.01 \mathrm{~m}, 0.05 \mathrm{~m}, 0.1 \mathrm{~m}$. As illustrated in Fig. 8.1, the length of one cycle is assumed to be $2 \ell$. This value is used to approximate the cycle time period $\tau$ by $\tau=2 \ell / V_{0}^{\text {cr }}$ after the value of $V_{0}^{\text {cr }}$ is evaluated by the optimization.

\begin{tabular}{|c|c|c|c|c|c|}
\hline \multicolumn{6}{|c|}{ Material constants } \\
\hline$E$ & $\nu$ & $m$ & $G_{\mathrm{C}} / \rho($ Set $)$ & h and Page 1974) & $C$ \\
\hline \multirow{4}{*}{$10^{9} \mathrm{~N} / \mathrm{m}^{2}$} & 0.3 & $0.08 \mathrm{~kg} / \mathrm{m}^{2}$ & $10 \mathrm{Jm} / \mathrm{kg}$ & & $10^{-14}$ \\
\hline & & \multicolumn{4}{|c|}{ Geometric constants } \\
\hline & & $\begin{array}{ll}\ell & 2 b\end{array}$ & $h$ & $\beta$ & \\
\hline & & $0.1 \mathrm{~m}$ & $10^{-4} \mathrm{~m}$ & 1.12 & \\
\hline
\end{tabular}

Table 8.1 Physical parameters used in the numerical examples.

In Fig. 8.3, the dimensionless performance function (8.22) is plotted for $k=2.5,3,3.5$. It is seen that the value of optimal tension $\tilde{T}_{0}^{*}$ is increased with increasing $k$.

In Tables 8.2 and 8.3, the results of the nondimensional optimization problem (8.22-8.23) are shown for the considered values of parameters $k$ and $a_{0}$. In Table 8.2 , the values of the productivity function $\tilde{J}$ at the optimum are shown. 


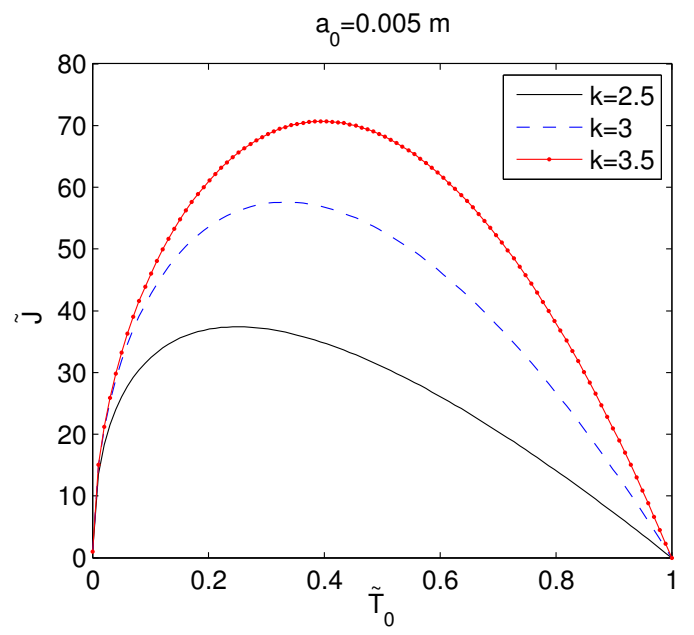

Fig. 8.3 Performance dependence on tension (dimensionless quantities). The dimensionless parameters and functions are presented in (8.19-8.21) and (8.22).

An increase in the length of the initial crack $a_{0}$ is seen to decrease productivity. The values of productivity seem to increase when $k$ is increased. However, one must take into account that also $J_{0}$, in (8.16-8.17), depends on $k$, which affects the actual productivity $J=J_{0} \tilde{J}$.

In Table 8.3, the optimal values of the dimensionless tension $\tilde{T}_{0}^{*}$ are shown. It is seen that the optimal dimensionless tension values slightly decrease when the crack length is increased.

Table 8.2 Dependence of the optimum of the performance $\tilde{J}$ on the Paris material constant $k$ and the initial crack length $a_{0}$.

\begin{tabular}{|c|c|c|c|c|}
\hline \multirow{2}{*}{$\tilde{J}^{*}$} & \multicolumn{4}{|l|}{$a_{0}(\mathrm{~m})$} \\
\hline & 0.005 & 0.01 & 0.05 & 0.1 \\
\hline$k=2.5$ & 37.4023 & 31.4527 & 21.0369 & 17.6920 \\
\hline$k=3$ & 57.5834 & 48.4230 & 32.3862 & 27.2358 \\
\hline$k=3.5$ & 70.6836 & 59.4390 & 39.7532 & 33.4308 \\
\hline
\end{tabular}

Since the actual optimal productivity, the actual tension, and the related critical speed and the critical number of cycles are of interest, these values were found at the optimum and are shown in Tables 8.4-8.5.

Note that several assumptions have been made. First, the Paris constant $C=10^{-14}$ is assumed to be independent of $k$, and both of the values are not measured for paper, but were chosen to be close to the typical values of some known materials. Secondly, the cycle time period $\tau$ is approximated assuming that one cycle length is $2 \ell$, that is, $\tau=2 \ell / V_{0}^{\text {cr }}$. 
Table 8.3 Dependence of optimal dimensionless tension $\tilde{T}_{0}^{*}$ on the Paris material constant $k$ and the initial crack length $a_{0}$.

\begin{tabular}{|c|c|c|c|c|}
\hline \multirow{2}{*}{$\tilde{T}_{0}^{*}$} & \multicolumn{4}{|l|}{$a_{0}(\mathrm{~m})$} \\
\hline & 0.005 & 0.01 & 0.05 & 0.1 \\
\hline$k=2.5$ & 0.2500 & 0.2499 & 0.2499 & 0.2498 \\
\hline$k=3$ & 0.3333 & 0.3333 & 0.3332 & 0.3332 \\
\hline$k=3.5$ & 0.3968 & 0.3968 & 0.3968 & 0.3967 \\
\hline
\end{tabular}

Table 8.4 Top: Dependence of the optimal tension $T_{0}^{*}(\mathrm{~N} / \mathrm{m})$ on the Paris material constant $k$ and the initial crack length $a_{0}(\mathrm{~m})$. Bottom: Critical velocity $V_{0}^{\mathrm{cr}}(\mathrm{m} / \mathrm{s})$ at the optimum, depending on the parameters $k$ and $a_{0}$.

\begin{tabular}{lllll}
\hline$T_{0}^{*}(\mathrm{~N} / \mathrm{m})$ & \multicolumn{3}{l}{$a_{0}(\mathrm{~m})$} & \\
\cline { 2 - 5 } & 0.005 & 0.01 & 0.05 & 0.1 \\
\hline$k=2.5$ & 504 & 356 & 159 & 113 \\
$k=3$ & 672 & 475 & 212 & 150 \\
$k=3.5$ & 800 & 565 & 253 & 179 \\
\hline
\end{tabular}

\begin{tabular}{|c|c|c|c|c|}
\hline \multirow{2}{*}{$V_{0}^{\mathrm{cr}}\left(T_{0}^{*}\right)(\mathrm{m} / \mathrm{s})$} & \multicolumn{4}{|l|}{$a_{0}(\mathrm{~m})$} \\
\hline & 0.005 & 0.01 & 0.05 & 0.1 \\
\hline$k=2.5$ & 79.352 & 66.727 & 44.623 & 37.523 \\
\hline$k=3$ & 91.628 & 77.051 & 51.529 & 43.332 \\
\hline$k=3.5$ & 99.979 & 84.073 & 56.226 & 47.282 \\
\hline
\end{tabular}

Table 8.5 Top: The number of cycles $n^{\text {cr }}$ at the optimum, depending on the parameters $k$ and $a_{0}$. Bottom: Dependence of the optimum of the performance $J(\mathrm{~kg})$ on the Paris material constant $k$ and the initial crack length $a_{0}(\mathrm{~m})$.

\begin{tabular}{lllll}
\hline$n^{\mathrm{cr}}\left(T_{0}^{*}\right)$ & $a_{0}(\mathrm{~m})$ & & & \\
\cline { 2 - 5 } & 0.005 & 0.01 & 0.05 & 0.1 \\
\hline$k=2.5$ & 757,300 & 636,834 & 425,943 & 358,216 \\
$k=3$ & 30,130 & 21,306 & 9,529 & 6,738 \\
$k=3.5$ & 1,348 & 801 & 239 & 142 \\
\hline \multicolumn{5}{c}{} \\
\hline \multirow{2}{*}{$J^{*}(\mathrm{~kg})$} & $a_{0}(\mathrm{~m})$ & & & \\
& 0.005 & 0.01 & 0.05 & 0.1 \\
\hline$k=2.5$ & 121,168 & 101,894 & 68,151 & 57,315 \\
$k=3$ & 4,821 & 3,409 & 1,525 & 1,078 \\
$k=3.5$ & 216 & 128 & 38 & 23 \\
\hline
\end{tabular}


The actual optimal tension $T_{0}^{*}$ is calculated from (8.19), that is $T_{0}^{*}=$ $T_{0}^{\mathrm{M}} \tilde{T}_{0}^{*}$. Since $T_{0}^{\mathrm{M}}$ depends only on fixed values, and the material parameters in $T_{0}^{\mathrm{M}}$ are measured and known for paper materials, the results for the actual optimal tension, shown in Table 8.4, left, are comparable and quite reliable. The results for the optimal tension $T_{0}^{*}$ are also illustrated as a colorsheet in Fig. 8.4.

In Table 8.4, right, the critical velocities corresponding to the optimal values of tension $V_{0}^{\text {cr }}\left(T_{0}^{*}\right)$ are shown. The values of velocities can be calculated directly from (8.12) using the values in Table 8.4a. As expected, the velocities decrease as $a_{0}$ is increased.

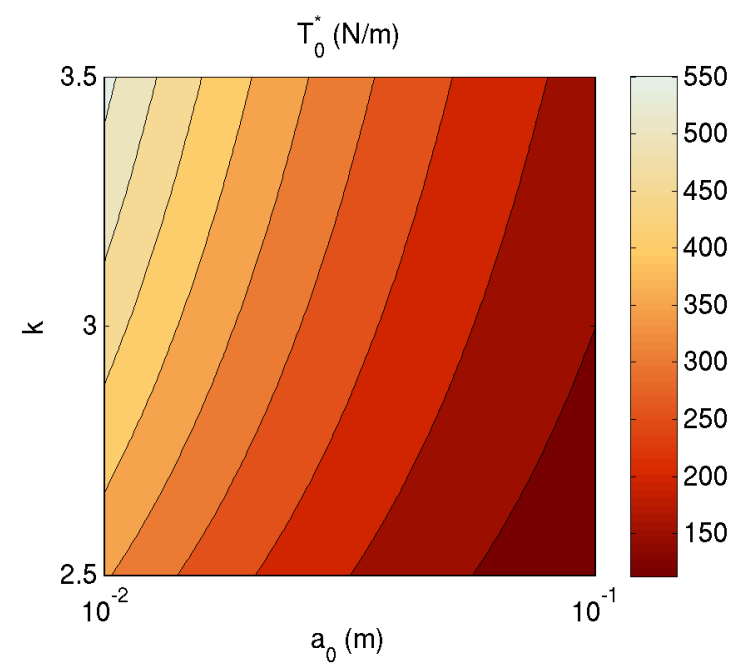

Fig. 8.4 A colorsheet showing the dependence of the optimal tension $T_{0}^{*}(\mathrm{~N} / \mathrm{m})$ on the parameters $k$ (Paris material constant) and $a_{0}$ (initial crack length). Note the logarithmic scale of $a_{0}$.

The actual optimal number of cycles $n^{\mathrm{cr}}\left(T_{0}^{*}\right)$ and the actual optimal productivity $J^{*}$ are more difficult to predict, since they depend on the Paris constant $C$, which is not known for paper materials. As mentioned above, the same value of $C$, namely $C=10^{-14}$, is used for all investigated values of $k$, which might not be reasonable. Since the value of $\kappa_{0}$ defined in (8.5) is large (the numerical value of $\kappa_{0}$ is larger than unity), then $\kappa_{0}^{k}$ increases with the increase in $k$. Keeping $C$ constant, we see from (8.5) that the crack growth rate may be larger for a large value of $k$, depending on the value of $a^{k / 2}$, which is small. This means that the number of cycles may be the smaller the greater the value of $k$ is, which can also be seen from (6.11): the greater the value of $k$, the smaller the value of $A$. In the results in Table 8.5, top, it can be seen that the effect of $\kappa_{0}$ is large, and the number of cycles at the optimum decreases remarkably when $k$ is increased. This also results 
in a decrease in the optimal productivity $J^{*}$, which is shown in Table 8.5, bottom.

Comparing the results in Tables 8.2 and 8.5, top, we therefore make no conclusion about the effect of $k$ on the actual performance $J^{*}$. The qualitative result of the decrease in performance $J^{*}$ when $a_{0}$ is increased is, however, plausible.

\subsection{Pareto optimal solutions for good runnability}

In this section, we seek an optimal in-plane tension that maximizes a performance vector function consisting of the critical velocity, the number of cycles before fracture and process effectiveness. The considered problem of multiple objectives is called a multi-objective optimization (multi-objective programming or multi-criteria optimization) problem. Necessary conditions for the optimality of the maximized vector function are derived, and the Pareto optimal solutions are found analytically for some example cases.

As was seen above, optimal magnitude of tension is essential for safe conditions in systems with axially travelling material. Seeking the optimal tension but having several objectives, such as high material longevity, transport velocity and productivity, we encounter a multi-objective optimization problem. Extensive literature reviews on multi-objective optimization are provided by White (1990) and Miettinen (1994). For a historical review of the origin and development of multicriteria optimization, refer to Stadler (1979). For surveys of concepts and methods of multi-objective optimization, see Chankong and Haimes (1983) and Steuer (1986).

Below, we will derive the multiobjective optimization problem consisting of maximizing the critical plate velocity, the longevity (critical number of loading cycles) and the productivity with respect to the value of in-plane tension. We concentrate especially on paper making productivity, though the analysis is also applicable to any other analogous processes.

The obtained objective vector function is transformed into a scalar objective function using the weighting method. For several important subproblems, the optimal value of tension is found analytically in the Pareto sense with respect to the other problem parameters.

\subsubsection{Multicriteria optimization}

We consider again an axially moving elastic plate which is travelling between a system of rollers. See Fig. 8.1.

All the plate elements 


$$
\Omega_{i} \equiv\left\{(x, y) \in \mathbb{R}^{2} \mid i \ell<x<(i+1) \ell,-b<y<b\right\}, \quad i=0,1,2, \ldots
$$

are subjected to homogeneous (in the $y$ direction) tension $T$ acting in the $x$ direction. The sides

$$
\{x=i \ell, \quad-b \leq y \leq b\} \quad \text { and } \quad\{x=(i+1) \ell, \quad-b \leq y \leq b\}
$$

are simply supported and the sides

$$
\{y=-b, \quad i \ell \leq x \leq(i+1) \ell\} \quad \text { and } \quad\{y=b, \quad i \ell \leq x \leq(i+1) \ell\}
$$

are free of tractions.

We present a productivity criterion (performance function) with the help of the plate velocity $V_{0}$ and the process time $t_{\mathrm{f}}$ :

$$
M=m_{0} V_{0} t_{\mathrm{f}}, \quad m_{0}=2 b m .
$$

In (8.28), the velocity $V_{0}$ is taken from the safe interval

$$
0<V_{0}<V_{0}^{\text {cr }}
$$

where $V_{0}^{\text {cr }}$ is the critical buckling velocity that is also taken as a criterion of the considered process:

$$
J_{\mathrm{V}} \equiv V_{0}^{\mathrm{cr}}
$$

where $V_{0}^{\text {cr }}$ is expressed by (8.12).

A safe interval for the safe functioning time (number of cycles) is written as

$$
0<t_{\mathrm{f}}<t_{\mathrm{f}}^{\mathrm{cr}} \quad \text { or } \quad 0<n<n^{\mathrm{cr}},
$$

where $t_{\mathrm{f}}^{\mathrm{cr}}$ and $n^{\mathrm{cr}}$ are, respectively, the time interval and the total number of cycles before fatigue fracture.

For a small cycle time period $\tau$ and a big number of cycles $n$, we assume that $t_{\mathrm{f}} \approx n \tau$.

We will consider the critical number of cycles as a safety function $J_{\mathrm{N}}$, i.e.,

$$
J_{\mathrm{N}} \equiv n^{\mathrm{cr}}
$$

where $n^{\mathrm{cr}}$ is given by (8.9), or (8.11) in the case of $k=2$. The productivity criterion $M^{\mathrm{cr}}$ is also considered as a problem function

$$
J_{\mathrm{M}} \equiv M^{\mathrm{cr}},
$$

where $M^{\text {cr }}$ is given by $(8.28)$ with critical parameter values. We have

$$
J_{\mathrm{M}}=m_{0} J_{\mathrm{V}} t_{\mathrm{f}}^{\mathrm{cr}}=m_{0} \tau J_{\mathrm{V}} J_{\mathrm{N}} .
$$


Note that the functions $J_{\mathrm{V}}, J_{\mathrm{N}}$ and $J_{\mathrm{M}}$ defined in (8.29), (8.30) and (8.31) depend on the value of in-plane average tension $T_{0}$ :

$$
\begin{aligned}
& J_{\mathrm{V}}=J_{\mathrm{V}}\left(T_{0}\right), \\
& J_{\mathrm{N}}=J_{\mathrm{N}}\left(T_{0}\right), \\
& J_{\mathrm{M}}=J_{\mathrm{M}}\left(T_{0}\right) .
\end{aligned}
$$

Using the limit velocity $V_{0}$, longevity $n$ and runnability effectiveness $M$ criteria, presented in the previous section, we may consider the following vector function

$$
J=\left\{\begin{array}{l}
J_{\mathrm{V}}\left(T_{0}\right) \\
J_{\mathrm{N}}\left(T_{0}\right) \\
J_{\mathrm{M}}\left(T_{0}\right)
\end{array}\right\}=\left\{\begin{array}{c}
V_{0}^{\mathrm{cr}}\left(T_{0}\right) \\
n^{\mathrm{cr}}\left(T_{0}\right) \\
M^{\mathrm{cr}}\left(T_{0}\right)
\end{array}\right\} .
$$

Now, we formulate the multicriteria (multiobjective) optimization problem. It is required to determine the optimal value $T_{0}^{*}$ of in-plane tension $T_{0}$ that gives a maximum of the considered vector function, i.e.

$$
J^{*}=J\left(T_{0}^{*}\right)=\max _{T_{0}} J\left(T_{0}\right) .
$$

The values in (8.33) and (8.34) are determined with the help of the corresponding formulas and relations presented in Sects. 8.1.1 and 8.1.2.

The max operation in (8.34) is considered in the Pareto sense. It is:

$$
T_{0}^{*}=\arg \max _{T_{0}} J\left(T_{0}\right)
$$

if there is no other value $\hat{T}_{0}$, such that

$$
J_{i}\left(\hat{T}_{0}\right) \geq J_{i}\left(T_{0}^{*}\right), \quad i=\mathrm{V}, \mathrm{N}, \mathrm{M},
$$

and the following rigorous inequality is satisfied for at least one component criterion:

$$
J_{j}\left(\hat{T}_{0}\right)>J_{j}\left(T_{0}^{*}\right) .
$$

To solve this multiobjective optimization problem, we apply the weighting method. We formulate the preference function as a sum of the single objective functionals $J_{\mathrm{V}}, J_{\mathrm{N}}, J_{\mathrm{M}}$ associated with the weighting factors $C_{\mathrm{V}}, C_{\mathrm{N}}, C_{\mathrm{M}}$ :

$$
J_{\mathrm{C}}=C_{\mathrm{V}} J_{\mathrm{V}}+C_{\mathrm{N}} J_{\mathrm{N}}+C_{\mathrm{M}} J_{\mathrm{M}},
$$

and we suppose that

$$
\begin{gathered}
C_{\mathrm{V}} \geq 0, \quad C_{\mathrm{N}} \geq 0, \quad C_{\mathrm{M}} \geq 0, \\
C_{\mathrm{V}}+C_{\mathrm{N}}+C_{\mathrm{M}}=1 .
\end{gathered}
$$


We will consider the multiobjective optimization problem of finding the optimal in-plane tension $T_{0}^{*}$ separately for different particular cases.

For convenience of performing the analysis and for reduction of characteristic parameters, we introduce the following values with tildes

$$
\begin{aligned}
\tilde{J}_{\mathrm{V}} & =\frac{J_{\mathrm{V}}}{J_{\mathrm{V}}^{0}}, & J_{\mathrm{V}}^{0} & =\sqrt{\frac{K_{\mathrm{C}} h}{m \beta \sqrt{\pi a_{0}}}}, \\
\tilde{J}_{\mathrm{N}} & =\frac{J_{\mathrm{N}}}{J_{\mathrm{N}}^{0}}, & J_{\mathrm{N}}^{0} & =\frac{2}{(k-2) C \kappa_{0}^{k} a_{0}^{(k-2) / 2}}, \\
\tilde{J}_{\mathrm{M}} & =\frac{J_{\mathrm{M}}}{J_{\mathrm{M}}^{0}}, & J_{\mathrm{M}}^{0} & =m_{0} \tau J_{\mathrm{V}}^{0} J_{\mathrm{N}}^{0},
\end{aligned}
$$

and represent the criterion functions as

$$
\begin{aligned}
& \tilde{J}_{\mathrm{V}}=\left(\tilde{T}_{0}+d\right)^{1 / 2}, \\
& {\tilde{J_{\mathrm{N}}}}=1-\tilde{T}_{0}^{k-2}, \\
& {\tilde{J_{\mathrm{M}}}}^{k}=\tilde{J}_{\mathrm{V}}{\tilde{J_{\mathrm{N}}}}
\end{aligned}
$$

using the dimensionless values and problem parameters:

$$
\tilde{T}_{0}=\frac{\beta \sqrt{\pi a_{0}}}{K_{\mathrm{C}} h} T_{0}, \quad d=\frac{\gamma_{*}^{2} \pi^{2} D \beta \sqrt{\pi a_{0}}}{l^{2} K_{\mathrm{C}} h}, \quad 0 \leq \tilde{T}_{0} \leq 1
$$

\subsubsection{Maximizing critical velocity and safety criterion}

We consider the case of maximization of the velocity criterion $\tilde{J}_{\mathrm{V}}$ and the safety criterion $\tilde{J}_{\mathrm{N}}$ when $k=3$. In this case, we have

$$
\begin{gathered}
\tilde{J}_{1} \equiv C_{\mathrm{V}} \tilde{J}_{\mathrm{V}}+C_{\mathrm{N}} \tilde{J}_{\mathrm{N}}, \\
C_{\mathrm{V}}+C_{\mathrm{N}}=1 .
\end{gathered}
$$

Let us study the solution of (8.39) with respect to the weight $C_{\mathrm{N}}$. Now, the optimization problem is (note $C_{\mathrm{V}}=1-C_{\mathrm{N}}$ )

$$
\max _{0 \leq \tilde{T}_{0} \leq 1}\left(1-C_{\mathrm{N}}\right)\left(\tilde{T}_{0}+d\right)^{1 / 2}+C_{\mathrm{N}}\left(1-\tilde{T}_{0}\right) .
$$

The object function in (8.40) is concave, so the use of the weighting method is justifiable for finding the Pareto optimal solutions.

Depending on the value of the weight $C_{\mathrm{N}}$, the optimal value of the dimensionless tension $\tilde{T}_{0}^{*}$, which in this case can be found at the at the zero of the derivative of the objective function $\tilde{J}_{1}$, is 


$$
\begin{array}{rlrl}
0 & \leq C_{\mathrm{N}} \leq \frac{1}{1+2 \sqrt{d+1}}: & & \tilde{T}_{0}^{*}=1, \\
\frac{1}{1+2 \sqrt{d+1}}<C_{\mathrm{N}}<\frac{1}{1+2 \sqrt{d}}: & & \tilde{T}_{0}^{*}=\left(\frac{1-C_{\mathrm{N}}}{2 C_{\mathrm{N}}}\right)^{2}-d, \\
\frac{1}{1+2 \sqrt{d}} \leq C_{\mathrm{N}} \leq 1: & & \tilde{T}_{0}^{*}=0 .
\end{array}
$$

Consequently, the values of the component functions are found in the following form:

$$
\begin{aligned}
& \tilde{J}_{\mathrm{V}}=\sqrt{\tilde{T}_{0}^{*}+d}=\frac{1-C_{\mathrm{N}}}{2 C_{\mathrm{N}}}, \\
& \tilde{J}_{\mathrm{N}}=1-\tilde{T}_{0}^{*}=1+d-\left(\frac{1-C_{\mathrm{N}}}{2 C_{\mathrm{N}}}\right)^{2},
\end{aligned}
$$

and, for the considered problem of critical velocity and longevity maximization, the Pareto front $(\mathrm{PF})$ of the optimal solution is given by the equation

$$
\tilde{J}_{\mathrm{N}}=1+d-\tilde{J}_{\mathrm{V}}^{2},
$$

where

$$
\tilde{J}_{\mathrm{V}} \in[\sqrt{d}, \sqrt{1+d}] .
$$

The Pareto front is represented in Fig. 8.5.

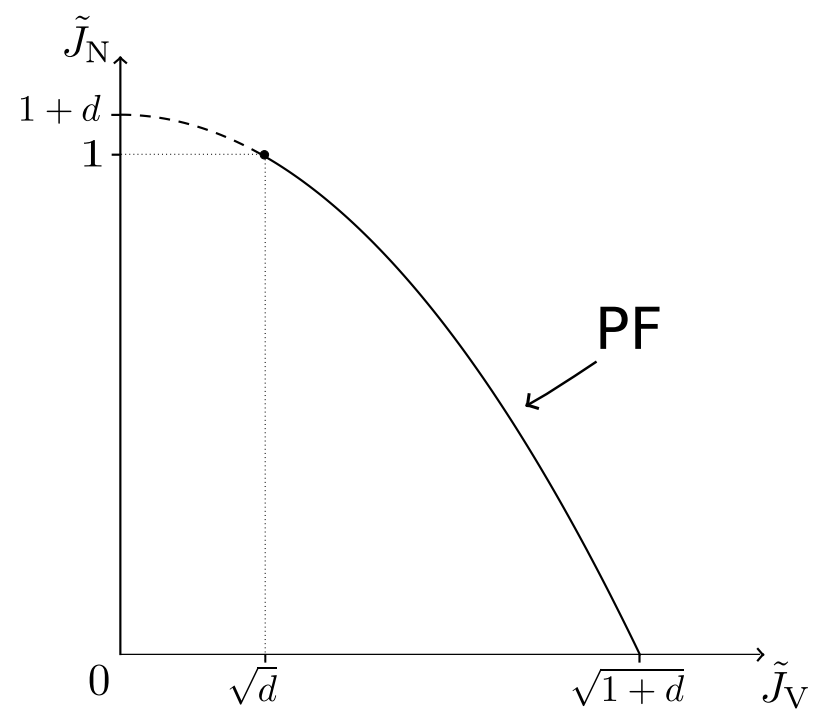

Fig. 8.5 The Pareto front (PF) for the problem of maximizing the critical velocity $\tilde{J}_{\mathrm{V}}$ and safety criterion $\tilde{J}_{\mathrm{N}}$. See (8.37) and (8.41). A schematic figure. 


\subsubsection{Maximizing critical velocity and process effectiveness}

Consider now another case, where we maximize the critical velocity criterion $\tilde{J}_{\mathrm{V}}$ and the process effectiveness criterion $\tilde{J}_{\mathrm{M}}$. We discuss again the case with $k=3$. In this case, the weighting method problem is

$$
\begin{gathered}
\tilde{J}_{2} \equiv C_{\mathrm{V}} \tilde{J}_{\mathrm{V}}+C_{\mathrm{M}} \tilde{J}_{\mathrm{M}}, \\
C_{\mathrm{V}}+C_{\mathrm{M}}=1,
\end{gathered}
$$

so that we study

$$
\max _{0 \leq \tilde{T}_{0} \leq 1}\left[C_{\mathrm{V}}\left(\tilde{T}_{0}+d\right)^{1 / 2}+C_{\mathrm{M}}\left(\tilde{T}_{0}+d\right)^{1 / 2}\left(1-\tilde{T}_{0}\right)\right] .
$$

The object function in (8.42) is concave. Now, the extremum condition is

$$
\begin{aligned}
\frac{\mathrm{d} \frac{\tilde{J}_{2}}{\mathrm{~d} \tilde{T}_{0}}}{} & =C_{\mathrm{V}} \frac{\mathrm{d} \frac{\tilde{J}_{\mathrm{V}}}{\mathrm{d} \tilde{T}_{0}}+C_{\mathrm{M}} \frac{\mathrm{d} \tilde{J}_{\mathrm{M}}}{\mathrm{d} \tilde{T}_{0}}}{} \\
& =C_{\mathrm{V}} \frac{\mathrm{d} \tilde{J}_{\mathrm{V}}}{\mathrm{d} \tilde{T}_{0}}+C_{\mathrm{M}}\left(\tilde{J}_{\mathrm{N}} \frac{\mathrm{d} \tilde{J}_{\mathrm{V}}}{\mathrm{d} \tilde{T}_{0}}+\tilde{J}_{\mathrm{V}} \frac{\mathrm{d} \tilde{J}_{\mathrm{N}}}{\mathrm{d} \tilde{T}_{0}}\right) \\
& =0 .
\end{aligned}
$$

The solution of the problem is studied with respect to the weight $C_{\mathrm{M}}$ (note again $\left.C_{\mathrm{V}}=1-C_{\mathrm{M}}\right)$. By (8.43), it is found that the optimal value for the dimensionless tension $\tilde{T}_{0}^{*}$ depends on $C_{\mathrm{M}}$ as follows:

$$
\begin{aligned}
0 & \leq C_{\mathrm{M}} \leq \frac{1}{2 d+3}: & \tilde{T}_{0}^{*} & =1 \\
\frac{1}{2 d+3} & <C_{\mathrm{M}} \leq 1: & \tilde{T}_{0}^{*} & =\frac{1-2 d C_{\mathrm{M}}}{3 C_{\mathrm{M}}} .
\end{aligned}
$$

For the optimized functionals $\tilde{J}_{\mathrm{V}}$ and $\tilde{J}_{\mathrm{M}}$, we have

$$
\begin{aligned}
& \tilde{J}_{\mathrm{V}}^{2}=\frac{1}{3}\left(\frac{1}{C_{\mathrm{M}}}+d\right) \\
& \tilde{J}_{\mathrm{M}}=\frac{1}{3}\left(2 d+3-\frac{1}{C_{\mathrm{M}}}\right) \sqrt{\frac{1}{3}\left(\frac{1}{C_{\mathrm{M}}}+d\right)} .
\end{aligned}
$$

The Pareto front of the problem under consideration is described by the equation

$$
\tilde{J}_{\mathrm{M}}=(1+d) \tilde{J}_{\mathrm{V}}-\tilde{J}_{\mathrm{V}}^{3}
$$

defined on the interval 


$$
\tilde{J}_{\mathrm{V}} \in[\sqrt{(1+d) / 3}, \sqrt{1+d}]
$$

and is shown in Fig. 8.6.

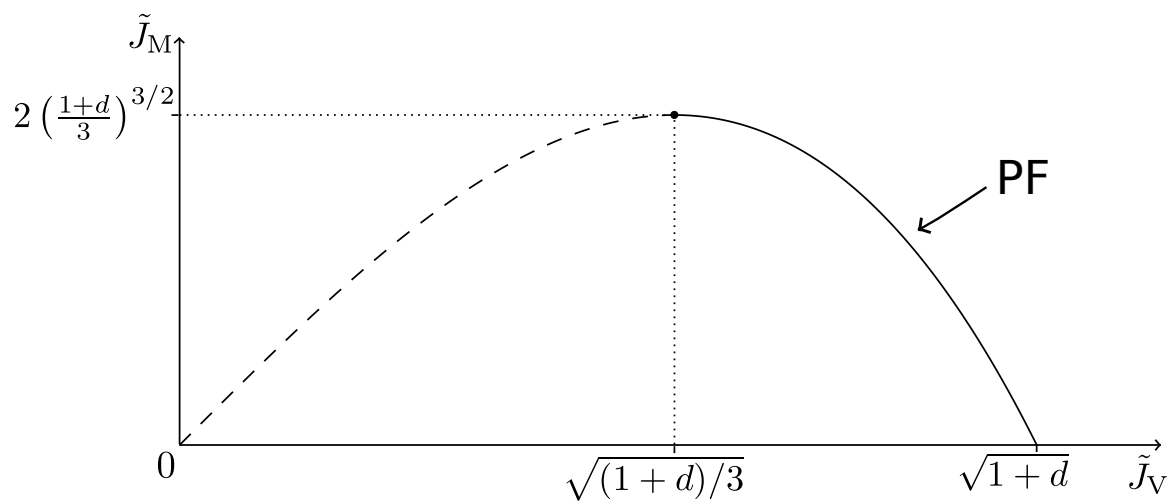

Fig. 8.6 The Pareto front $(\mathrm{PF})$ for the problem of maximizing the critical velocity $\tilde{J}_{\mathrm{V}}$ and the criterion of process effectiveness $\tilde{J}_{\mathrm{M}}$. See (8.37) and (8.44). A schematic figure.

\subsubsection{Maximizing safety and process effectiveness}

As a third case, we study the maximization of the safety criterion $\tilde{J}_{\mathrm{N}}$ and the process effectiveness criterion $\tilde{J}_{\mathrm{M}}$ when $k=3$. We have

$$
\begin{gathered}
\tilde{J}_{3} \equiv C_{\mathrm{N}} \tilde{J}_{\mathrm{N}}+C_{\mathrm{M}} \tilde{J}_{\mathrm{M}}, \\
C_{\mathrm{N}}+C_{\mathrm{M}}=1,
\end{gathered}
$$

and the optimization problem reads

$$
\max _{0 \leq \tilde{T}_{0} \leq 1}\left[C_{\mathrm{N}}\left(1-\tilde{T}_{0}\right)+\left(1-C_{\mathrm{N}}\right)\left(\tilde{T}_{0}+d\right)^{1 / 2}\left(1-\tilde{T}_{0}\right)\right] .
$$

Also the object function $\tilde{J}_{3}$ is concave. We study the problem (8.45) with respect to the weight $C_{\mathrm{N}}$. Now the optimal value of the dimensionless tension $\tilde{T}_{0}^{*}$ depends on $C_{\mathrm{N}}$ in the following way: 


$$
\begin{array}{rlrl}
0 \leq C_{\mathrm{N}}<\frac{1-2 d}{1-2 d+2 \sqrt{d}}: & \tilde{T}_{0}^{*} & =\frac{2}{9}\left(\alpha^{2}-3 d+3 / 2\right. \\
& \left.-\alpha \sqrt{\alpha^{2}+3 d+3}\right), \\
\frac{1-2 d}{1-2 d+2 \sqrt{d}} \leq C_{\mathrm{N}} \leq 1: & \tilde{T}_{0}^{*} & =0
\end{array}
$$

where

$$
\alpha \equiv \frac{C_{\mathrm{N}}}{C_{\mathrm{M}}}=\frac{C_{\mathrm{N}}}{1-C_{\mathrm{N}}} .
$$

In this case, the Pareto front is given by

$$
\tilde{J}_{\mathrm{M}}=\tilde{J}_{\mathrm{N}} \sqrt{1+d-\tilde{J}_{\mathrm{N}}}, \quad \tilde{J}_{\mathrm{N}} \in\left[\frac{2}{3}(1+d), 1\right] .
$$

See Fig. 8.7.

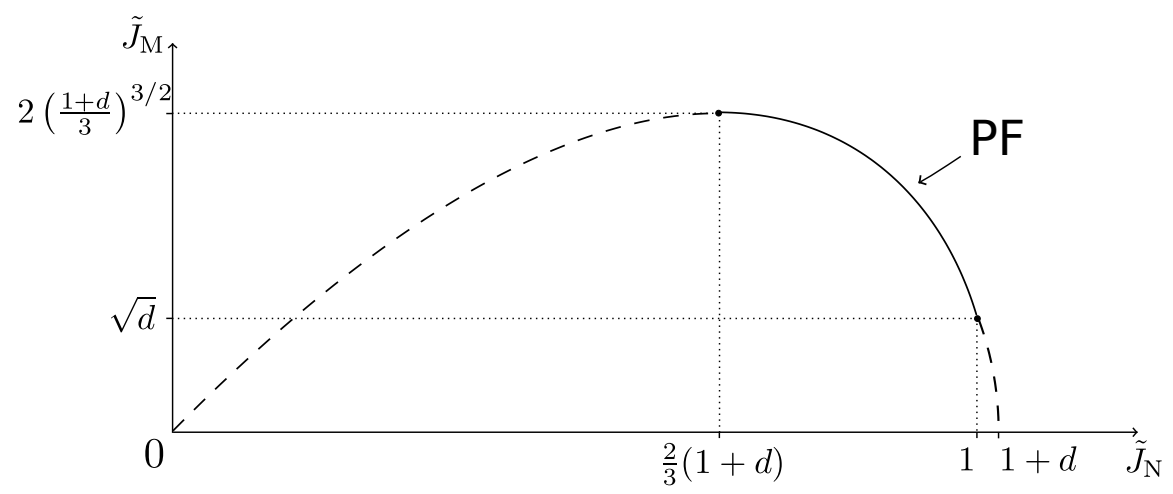

Fig. 8.7 The Pareto front (PF) for the problem of maximizing the critical number of cycles $\tilde{J}_{\mathrm{N}}$ and the criterion of process effectiveness $\tilde{J}_{\mathrm{M}}$. See (8.37) and (8.47). A schematic figure.

Finally, note that the maximum of (8.35) in the case $C_{\mathrm{M}}=1$ and $C_{\mathrm{V}}=$ $C_{\mathrm{N}}=0$ is found above by solving the problems (8.42) and (8.45). Then,

$$
\tilde{J}_{\mathrm{V}}=\sqrt{(1+d) / 3} \quad \text { and } \quad \tilde{J}_{\mathrm{N}}=\frac{2}{3}(1+d)
$$

which is also a Pareto optimal solution for the problem (8.40) confirmed by (8.41). 


\subsubsection{Some illustrations}

In the previous section, the multi-objective optimization problems of maximizing the critical velocity, maximizing the longevity and maximizing the process effectiveness were studied, and analytical results were found for some special subproblems.

The obtained analytical results are illustrated numerically in this section. Parameter values (material and geometrical) are given in Table (8.6). The paper fracture toughness is $K_{\mathrm{C}}=\sqrt{G_{\mathrm{C}} E}$. The investigated critical crack length $a_{0}$ obtains the values $0.005 \mathrm{~m}, 0.01 \mathrm{~m}, 0.05 \mathrm{~m}$ and $0.1 \mathrm{~m}$.

Table 8.6 Physical parameters used in the numerical examples.

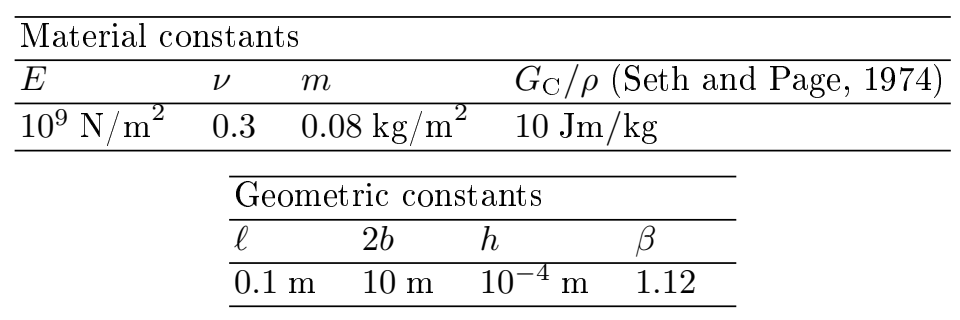

The Pareto fronts (8.41), (8.44) and (8.47) are illustrated in Fig. 8.8 when the initial crack length is $a_{0}=0.01 \mathrm{~m}$.

In Fig. 8.9, the optimal values of tension $T_{0}^{*}(\mathrm{~N} / \mathrm{m})$ for the problems $(8.40)$, (8.42) and (8.45) are plotted with respect to the weights $\left(C_{\mathrm{N}}, C_{\mathrm{M}}\right.$ and $C_{\mathrm{N}}$, respectively) and the initial length of the crack $a_{0}$.

In Fig. 8.9, top, we present the optimal values of tension $T_{0}^{*}$ when the velocity $J_{\mathrm{V}}$ and the longevity $J_{\mathrm{N}}$ are optimized. One may note that the even for a small crack size $\left(a_{0}=0.01\right)$, the optimal value of tension is almost zero, when the longevity is given a large weight $\left(C_{\mathrm{N}}>0.8\right)$. Weighting the velocity, the optimal tension obtains very large values $\left(T_{0}^{*} \sim 1400 \mathrm{~N} / \mathrm{m}\right)$. In other words, changing the weights radically changes the optimal result. In this case, it is difficult to decide how to weight the object functions.

In Fig. 8.9, middle, we weight the velocity function $J_{\mathrm{V}}$ against the process effectiveness function $J_{\mathrm{M}}$. In this case, it is noted that the length of the initial crack length significantly affects the optimal value of tension.

Figure 8.9, bottom, shows the third case, where the longevity $J_{\mathrm{N}}$ and the process effectiveness $J_{\mathrm{M}}$ are compared. Also here, it is seen that $a_{0}$ has an effect on the value of optimal tension, especially when the process effectiveness is weighted.

Note that the case $C_{\mathrm{M}}=1$ and $C_{\mathrm{V}}=C_{\mathrm{N}}=0$ is included in both middle and bottom parts of Fig. 8.9, giving the lowest values for tension in the middle figure and the highest values in the bottom figure. Analyzing these two subproblems helps us to make decisions on the weights to be selected. 

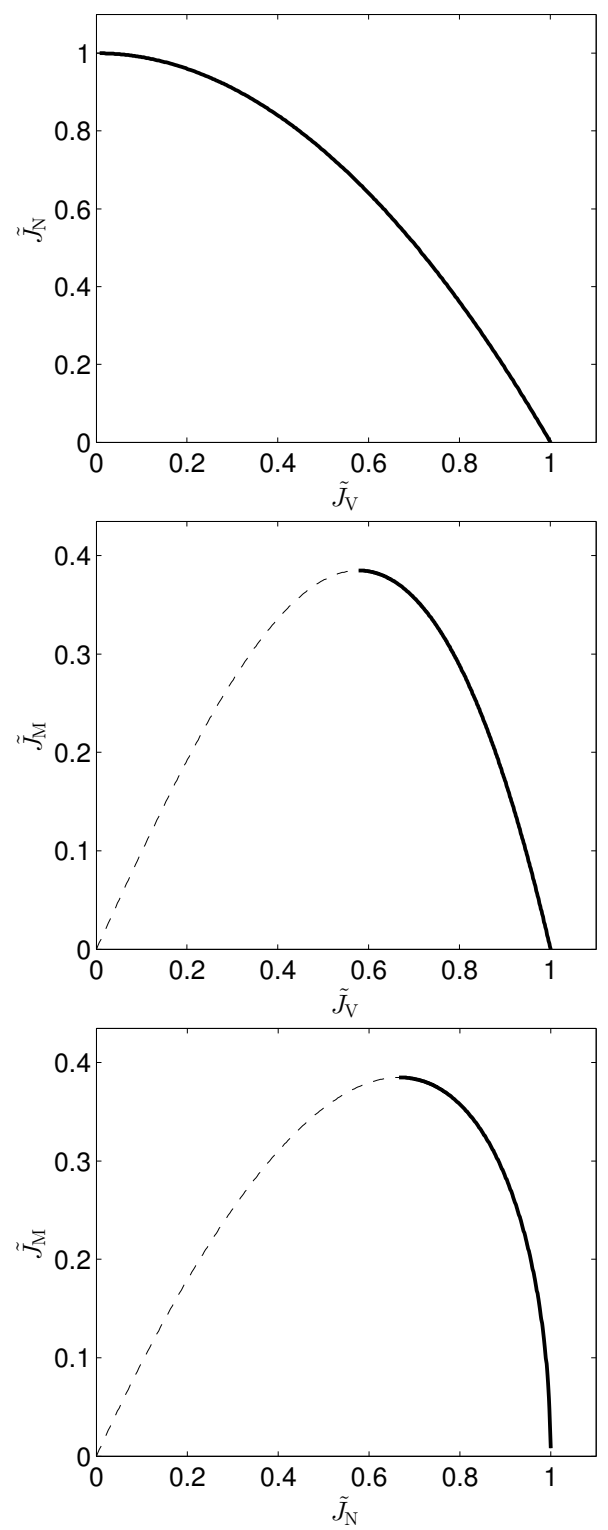

Fig. 8.8 Pareto fronts for the problems $\max \left\{\tilde{J}_{\mathrm{N}}, \tilde{J}_{\mathrm{V}}\right\}, \max \left\{\tilde{J}_{\mathrm{M}}, \tilde{J}_{\mathrm{V}}\right\}$, and $\max \left\{\tilde{J}_{\mathrm{M}}, \tilde{J}_{\mathrm{N}}\right\}$, respectively, in the case when the initial crack length $a_{0}=0.01 \mathrm{~m}$.

The optimum for process effectiveness gives some kind of reference value for the desired tension.

With the help of Fig. 8.9, we have chosen some values for the weights $C_{\mathrm{N}}$ and $C_{\mathrm{M}}$. The solutions are collected into Table 8.7. 

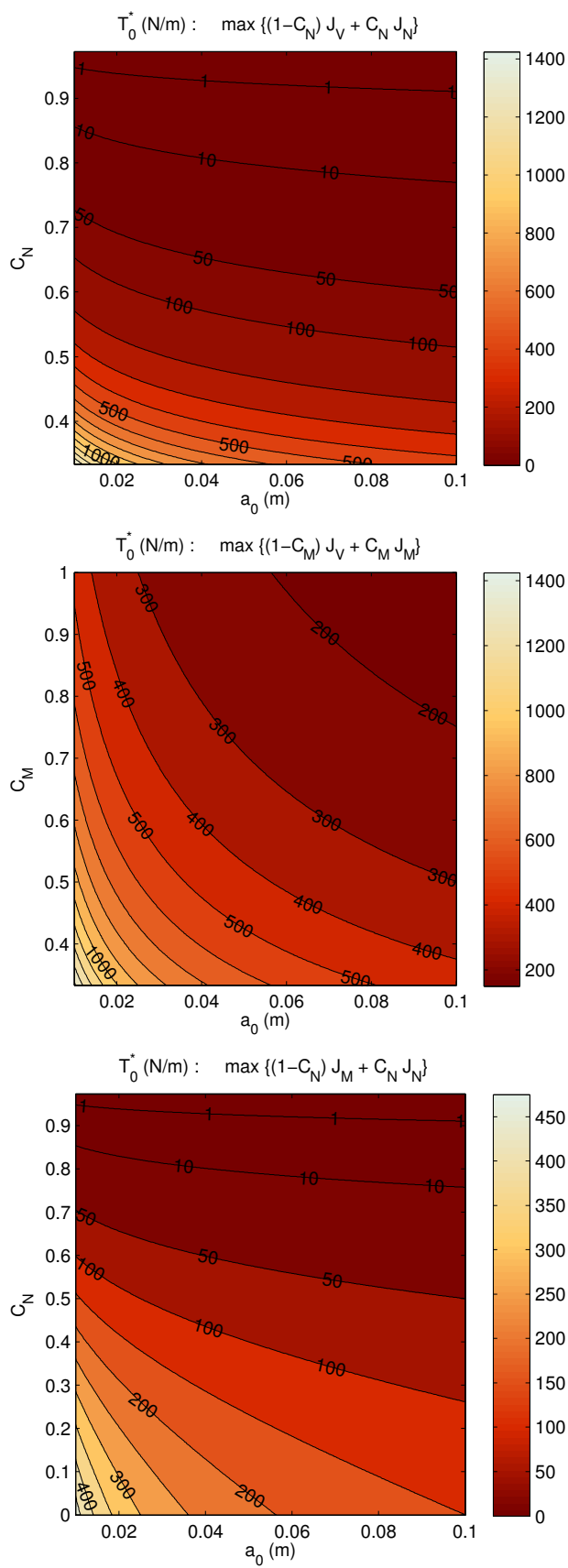

Fig. 8.9 Dependence of the optimal tension $T_{0}$ on the initial crack length $a_{0}$ and the weight $C_{\mathrm{N}}$ or $C_{\mathrm{M}}$. (Reproduced from Banichuk et al 2013) 
Table 8.7 Dependence of the optimal tension (dimensionless $\tilde{T}_{0}^{*}$ and dimensional $T_{0}^{*}$ ) on the selected weights for the three studied cases. The used initial crack length was $a_{0}=0.01$ m.(Banichuk et al 2013)

\begin{tabular}{|c|c|c|c|c|c|c|}
\hline \multirow{2}{*}{\multicolumn{4}{|c|}{$\overline{\arg \max \left\{C_{\mathrm{N}} J_{\mathrm{N}}+C_{\mathrm{V}} J_{\mathrm{V}}\right\}}$}} & \multicolumn{3}{|c|}{$\arg \max \left\{C_{\mathrm{M}} J_{\mathrm{M}}+C_{\mathrm{V}} J_{\mathrm{V}}\right\}$} \\
\hline & & & & \multirow{3}{*}{$\frac{\overline{C_{\mathrm{M}}}}{0.4}$} & \multirow{2}{*}{$\begin{array}{l}\tilde{T}_{0}^{*} \\
0.8333\end{array}$} & \multirow{3}{*}{$\begin{array}{l}T_{0}^{*}(\mathrm{~N} / \mathrm{m}) \\
1,187\end{array}$} \\
\hline \multicolumn{4}{|c|}{$\begin{array}{lll}C_{\mathrm{N}} & \tilde{T}_{0}^{*} & T_{0}^{*}(\mathrm{~N} / \mathrm{m})\end{array}$} & & & \\
\hline$\overline{0.4}$ & 0.5624 & 801 & & & & \\
\hline 0.5 & 0.2499 & 356 & & 0.6 & 0.0555 & 791 \\
\hline 0.6 & 0.1110 & 158 & & 0.0 & 0.4761 & $\begin{array}{l}191 \\
678\end{array}$ \\
\hline 0.7 & 0.0459 & 65 & & 08 & 0.4166 & 594 \\
\hline 0.8 & 0.0156 & 22 & & 09 & 03703 & 528 \\
\hline 0.9 & 0.0030 & 4 & & 1.0 & 0.3333 & 475 \\
\hline \multicolumn{7}{|c|}{$\arg \max \left\{C_{\mathrm{N}} J_{\mathrm{N}}+C_{\mathrm{M}} J_{\mathrm{M}}\right\}$} \\
\hline & & $C_{\mathrm{N}}$ & $T_{0}^{*}$ & \multicolumn{3}{|c|}{$T_{0}^{*}(\mathrm{~N} / \mathrm{m})$} \\
\hline & & $\overline{0.0}$ & 0.3333 & \multicolumn{3}{|c|}{475} \\
\hline & & 0.1 & 0.2932 & \multicolumn{3}{|c|}{418} \\
\hline & & 0.2 & 0.2500 & \multicolumn{3}{|c|}{356} \\
\hline & & 0.3 & 0.2042 & \multicolumn{3}{|c|}{291} \\
\hline & & 0.4 & 0.1571 & \multicolumn{3}{|c|}{224} \\
\hline & & 0.5 & 0.1111 & \multicolumn{3}{|c|}{158} \\
\hline & & 0.6 & 0.0695 & \multicolumn{3}{|c|}{99} \\
\hline & & 0.7 & 0.0364 & \multicolumn{3}{|l|}{52} \\
\hline & & 0.8 & 0.0143 & \multicolumn{3}{|l|}{20} \\
\hline & & 0.9 & 0.0030 & \multicolumn{3}{|l|}{4} \\
\hline
\end{tabular}

\subsection{Optimization with uncertainties}

In this section, we present a stochastic analysis of axially moving cracked elastic plates with uncertainties. The study is focused on instability and material fracture, which are the most serious threats to stable production of a papermachine. On these phenomena a change in tension magnitude has opposite effects. Increasing the magnitude of tension has a stabilizing effect but it may lead to growing of cracks. We will present an analysis to find the optimal value of velocity and tension for efficient product processing.

In last decades, the studies of runnability have been based on a deterministic approach. However, we know that in practice the values of different parameters are not known precisely and the process to be modelled usually includes random factors. At the last section of this book, we would like to raise awareness of this and offer one relatively simple approach to consider this issue.

From the application point of view, uncertainty occurs as, e.g., variation of tension, in space and time, in the press system of a papermachine, and defects on a paper web, which vary in their location, size, shape and orientation 
(Björklund and Svedjebrant 2009; Niskanen 2012). Another example is given by strength of paper which was found to obey the Weibull and Duxbury distributions by Salminen (2003). According to Uesaka (2004), the majority of web breaks in paper production are caused by tension variations, combined with strength variations of the paper web. Wathén (2003) discusses the effect of flaws of paper on web breaks and notes that even a seemingly perfect paper can fail at very low tensions due to stress concentrations caused by discontinuities, e.g., cuts and shives, inside the structure. Because of the stochastic structure of paper, it is difficult to predict occurences of flaws. Therefore we include uncertainty aspects in the model and study the problem of finding the optimal velocity from a probabilistic point of view.

There occur a large variety of defects in a paper web during its manufacturing process, but we concentrate on studying a plate with an initial crack at the edge, which can be considered the most usual case. Björklund and Svedjebrant (2009) have found that there is a higher density of defects at the edges of the paper, possibly as a result of greater variance of the steam box control at the edges. Smith (1995) classifies edge cracks as edge cuts or nicks that usually extend only a short distance. A fiber cut in the web or plate is defined as a typically short and straight cut that is located randomly, and is usually at an approximately right angle at the edge. Smith also lists several possible reasons for an occurrence of a broken edge, the list including dry edges, high sheet caliper at the edge, and web overlapping. A fiber cut is caused when a pulp fiber or shive that is less compactible than the rest of the web, passes through a high pressure nip.

In the following, the theoretical treatment is divided into two parts. In the first part, we assume the moving plate to have an initial crack of random length at the edge, and we formulate analytical expressions for the optimal tension and velocity of the plate. In the second study, the magnitude of homogeneous in-plane tension affecting the plate in the machine direction is assumed to be a random variable, and we derive a formula for the optimal velocity. In this part, the length of the crack is assumed to be constant.

The obtained analytical expressions are used for computing the optimal tension and the corresponding optimal velocity numerically. To do this, we use the log-normal distribution for the crack length and the in-plane tension is modelled with truncated normal distribution. The effect of changing the values of distribution parameters is illustrated. In the case of random crack length we will see that the optimal values decrease when the expected value and variance of the crack length increase. In the case of random tension, it is seen that the more the magnitude of tension is dispersed, the lower is the optimal tension. We will also illustrate the effect of changing the value of the admissible probabilites in the constraints. It will be seen that the optimal values increase when the probabilities increase. 


\subsubsection{Uncertainty in initial crack length}

We consider a rectangular elastic plate, which is supported by rollers at both ends and is moving at a constant velocity $V_{0}$. Denotation of this domain is the same as in the previous chapters of this book,

$$
\Omega \equiv\left\{(x, y) \in \mathbb{R}^{2} \mid 0<x<\ell,-b<y<b\right\}
$$

where $\ell$ and $b$ are prescribed parameters of length and width. The considered domain $\Omega$ is a representation of a thin isotropic elastic plate having constant thickness $h$, Poisson ratio $\nu$, Young modulus $E$, and bending rigidity $D=$ $E h^{3} /\left[12\left(1-\nu^{2}\right)\right]$. The mass of the plate per unit area is denoted by $m$. We assume that the plate is subjected to homogeneous tension $T_{0}$ acting in the $x$ direction. We also assume that the plate travels in the $x$ direction as usual. The supporting rollers are located at both ends of the plate, the other edges are free of traction. Schematic setup of the problem is presented in Fig. 8.10.

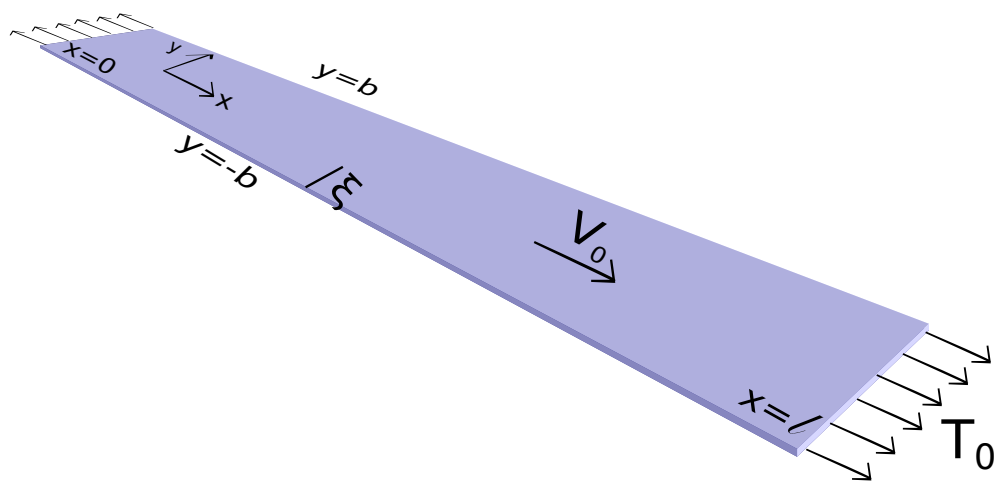

Fig. 8.10 A travelling elastic plate with a crack at the edge.

Suppose there is an initial crack of mode I (see Fig. 8.11) at the edge of the plate, and let $\xi$ be a positive valued random variable that describes the length of the crack. We consider the stress intensity factor (SIF) related to the crack and want to avoid the stress intensity factor reaching its critical value, known as the critical fracture toughness, at which the crack begins to propagate.

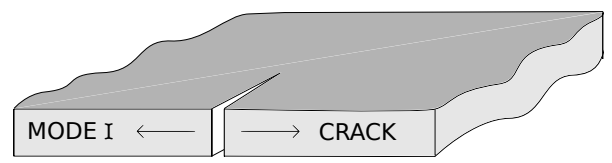

Fig. 8.11 Mode I crack (opening). 
We formulate an optimization problem using a similar approach as is presented in the book by Banichuk and Neittaanmäki (2010). We seek the maximal magnitude of velocity under the constraint that the probability of fracture is small. Letting $p \in(0,1)$ denote the probability of fracture that can be accepted, our optimization problem reads as

$$
\begin{aligned}
& \max _{T_{0}} V_{0}^{\mathrm{cr}}\left(T_{0}\right), \quad \text { such that } \\
& P\left(K_{\mathrm{I}} \geq K_{\mathrm{IC}}\right) \leq p,
\end{aligned}
$$

where $V_{0}^{\text {cr }}$, given by $(8.12)$, is the critical velocity, and $K_{\mathrm{IC}}$ is the critical fracture toughness of the considered material.

For the stress intensity factor we use the expression

$$
K_{\mathrm{I}}=\frac{\alpha(\xi) T_{0} \sqrt{\pi \xi}}{h},
$$

where $\alpha$ is a weight function that depends on the geometry of the domain, the ratio of $\xi$ and $b$ and the ratio of $\ell$ and $b$. The formula of $\alpha$ is given, e.g., in Perez (2004), Laham (1998) and Fett (1999). We assume $\alpha$ to be an increasing positive function of $\xi$ and, for simplicity, approximate it as a constant function $\alpha=1.12$ in this study.

To solve problem (8.49-8.50) we are looking for the maximal value of the tension $T_{0}$ that satisfies the inequality (8.50) and the equation of stress intensity factor (8.51). The constraint (8.50) is equal to

$$
P\left(\xi \geq g^{-1}\left(\frac{K_{\mathrm{IC}} h}{T_{0} \sqrt{\pi}}\right)\right) \leq p,
$$

where $g^{-1}$ is the inverse function of the function $g(\xi) \equiv \alpha(\xi) \sqrt{\xi}$. The inverse function exists, since $g$ is is strictly increasing due to the assumptions of $\alpha$. Further, the inequality above is equal to

$$
F_{\xi}\left(g^{-1}\left(\frac{K_{\mathrm{C}} h}{T_{0} \sqrt{\pi}}\right)\right) \geq 1-p
$$

where $F_{\xi}$ the cumulative distribution function of $\xi$. Assuming that $\xi$ has a continuous density function, the function $F_{\xi}$ is strictly increasing.

Denote

$$
\xi_{\mathrm{C}}=F_{\xi}^{-1}(1-p)
$$

The value $\xi_{\mathrm{C}}$ is the minimum of the set

$$
\left\{x: \quad F_{\xi}(x) \geq 1-p\right\} .
$$

Consider another value of crack length $\xi_{\mathrm{C}}^{*}>\xi_{\mathrm{C}}$. For the values of tension $T_{\mathrm{C}}$ and $T_{\mathrm{C}}^{*}$ that satisfy 


$$
\xi_{\mathrm{C}}=g^{-1}\left(\frac{h K_{\mathrm{IC}}}{T_{\mathrm{C}} \sqrt{\pi}}\right) \quad \text { and } \quad \xi_{\mathrm{C}}^{*}=g^{-1}\left(\frac{h K_{\mathrm{IC}}}{T_{C}^{*} \sqrt{\pi}}\right)
$$

it holds

$$
T_{C}>T_{C}^{*}
$$

Thus, the maximal value of $T_{0}$ satisfying (8.50) is found by the equation

$$
P\left(K_{\mathrm{I}} \geq K_{\mathrm{IC}}\right)=p
$$

and can be expressed as

$$
T^{\max }=\frac{h K_{\mathrm{IC}}}{\alpha\left(F_{\xi}^{-1}(1-p)\right) \sqrt{\pi F_{\xi}^{-1}(1-p)}},
$$

The solution of the optimization problem $(8.49-8.50)$ is

$$
\left(V_{0}\right)_{\mathrm{opt}}=V_{0}^{\mathrm{cr}}\left(T^{\mathrm{max}}\right),
$$

The optimal tension $T^{\text {max }}$ and the optimal velocity are computed numerically presuming a distribution for the crack length. For this, we have chosen the log-normal distribution. The probability density function of $\xi$ is

$$
f_{\xi}(x)=\frac{1}{x s_{1} \sqrt{2 \pi}} \exp \left(-\frac{\left(\ln \left(x / c_{1}\right)\right)^{2}}{2 s_{1}^{2}}\right), \quad x>0, \quad s_{1}>0, \quad c_{1}>0,
$$

and the cumulative distribution function is

$$
F_{\xi}(x)=N\left(\frac{\ln x-\ln c_{1}}{s_{1}}\right),
$$

where

$$
N(x)=\frac{1}{\sqrt{2 \pi}} \int_{-\infty}^{x} \exp \left(-\frac{t^{2}}{2}\right) \mathrm{d} t
$$

is the cumulative distribution function of the standard normal distribution.

\subsubsection{Uncertainty in tension}

In practice, in a paper machine the tension of the plate is generated by a velocity difference between rollers. It is very likely, that for mechanical reasons, the tension practically varies lightly between minimum and maximum values i.e in-plane tension fluctuates around a given positive constant $T_{0}$. In this section, we model this phenomenon by describing the tension as

$$
T \equiv T_{0}+\theta
$$


where $\theta$ is a random variable that has a cumulative distribution function $F_{\theta}$. As before, the plate is assumed to have an initial crack of mode I. The length of the crack is assumed to be known and equal to a constant:

$$
\xi=a, \quad a>0 .
$$

In this case, formulation of optimization problem consists of two parts. First, we seek the maximal value of $T_{0}$ satisfying

$$
P\left(K_{\mathrm{I}} \geq K_{\mathrm{IC}}\right) \leq q_{1}, \quad \text { where } q_{1} \in(0,1)
$$

is the admissible probability of fracture. Secondly, denoting the solution of inequality (8.64) by $T_{0}^{\max }$, we search for the maximal value of velocity $V_{0}$ under a constraint for instability:

$$
P\left(V_{0}>V_{0}^{\mathrm{cr}}\left(T_{0}^{\max }+\theta\right)\right) \leq q_{2}, \quad \text { where } q_{2} \in(0,1) .
$$

Here $q_{2}$ is the admissible probability of instability.

The stress intensity factor related to the crack satisfies

$$
K_{\mathrm{I}}=\frac{\alpha(a) \sqrt{\pi a}}{h} T=\frac{\alpha(a) \sqrt{\pi a}}{h}\left(T_{0}+\theta\right) .
$$

Noticing that

$$
\begin{aligned}
P\left(K_{\mathrm{I}} \geq K_{\mathrm{IC}}\right) & =P\left(\theta \geq \frac{h K_{\mathrm{IC}}}{\alpha(a) \sqrt{\pi a}}-T_{0}\right) \\
& =1-F_{\theta}\left(\frac{h K_{\mathrm{IC}}}{\alpha(a) \sqrt{\pi a}}-T_{0}\right),
\end{aligned}
$$

the constraint (8.64) is equal to

$$
F_{\theta}\left(\frac{h K_{\mathrm{IC}}}{\alpha(a) \sqrt{\pi a}}-T_{0}\right) \geq 1-q_{1} .
$$

Denote the maximal value of tension that satisfies the above inequality by $T_{0}^{\max }$. If the function $F_{\theta}$ does not depend on $T_{0}$, it is seen with similar reasoning as above that the maximal value of tension that satisfies the inequality above is

$$
T_{0}^{\max }=\frac{K_{\mathrm{IC}} h}{\alpha(a) \sqrt{\pi a}}-F_{\theta}^{-1}\left(1-q_{1}\right) .
$$

Furthermore, 


$$
\begin{aligned}
P\left(V_{0}>V_{0}^{\mathrm{cr}}\left(T_{0}^{\max }+\theta\right)\right) & =P\left(V_{0}>\sqrt{\frac{T_{0}^{\max }+\theta}{m}+\gamma_{*}^{2} \frac{\pi^{2} D}{m l^{2}}}\right) \\
& =P\left(\theta<m V_{0}^{2}-T_{0}^{\max }-\gamma_{*}^{2} \frac{\pi^{2} D}{l^{2}}\right) \\
& =F_{\theta}\left(m V_{0}^{2}-T_{0}^{\max }-\gamma_{*}^{2} \frac{\pi^{2} D}{l^{2}}\right)
\end{aligned}
$$

and we may write the inequality (8.65) as

$$
F_{\theta}\left(m V_{0}^{2}-T_{0}^{\max }-\gamma_{*}^{2} \frac{\pi^{2} D}{l^{2}}\right) \leq q_{2} .
$$

Let us denote $\theta_{\mathrm{C}}=F_{\theta}^{-1}\left(q_{2}\right)$, and consider another value $\theta_{\mathrm{C}}^{*}<\theta_{\mathrm{C}}$. The two values of tension variation, $\theta_{\mathrm{C}}$ and $\theta_{\mathrm{C}}^{*}$, satisfy $F_{\theta}\left(\theta_{\mathrm{C}}\right) \leq q_{2}$ and $F_{\theta}\left(\theta_{\mathrm{C}}^{*}\right) \leq q_{2}$. By noticing that

$$
V_{0}=\sqrt{\frac{1}{m}\left(T_{0}^{\max }+\frac{\gamma_{*}^{2} \pi^{2} D}{l^{2}}+\theta_{\mathrm{C}}\right)}>\sqrt{\frac{1}{m}\left(T_{0}^{\max }+\frac{\gamma_{*}^{2} \pi^{2} D}{l^{2}}+\theta_{\mathrm{C}}^{*}\right)} \equiv V_{0}^{*},
$$

we deduce that the maximal value of $V_{0}$ satisfying (8.64) and (8.65) is

$$
\left(V_{0}\right)_{\mathrm{opt}}=\sqrt{\frac{1}{m}\left(T_{0}^{\max }+\frac{\gamma_{*}^{2} \pi^{2} D}{l^{2}}+F_{\theta}^{-1}\left(q_{2}\right)\right)} .
$$

We assume $\theta$ to obey the truncated normal distribution with scale parameter $c_{\mathrm{t}}>0$ and location at 0 . The minimum and maximum values of the distribution are set as

$$
\theta_{\min }=-T_{0} \quad \text { and } \quad \theta_{\max }=T_{0} .
$$

Hence, the probability function of $\theta$ is symmetrical, and expected value $E(\theta)=0$. The cumulative distribution function of $\theta$ is

$$
F_{\theta}\left(x ; c_{\mathrm{t}}, T_{0}\right)=\frac{N\left(\frac{x}{c_{\mathrm{t}}}\right)-N\left(\frac{-T_{0}}{c_{\mathrm{t}}}\right)}{1-2 N\left(\frac{-T_{0}}{c_{\mathrm{t}}}\right)},
$$

where $N$ is the cumulative distribution function of the standard normal distribution as above. In (8.70), we have

$$
F_{\theta}(x)=F_{\theta}\left(x ; c_{\mathrm{t}}, T_{0}^{\max }\right) .
$$

In the following, we illustrate numerically the results obtained above. The chosen parameter values are shown in Table 8.8. The paper fracture toughness 
$K_{\mathrm{IC}}$ is calculated from the equation $K_{\mathrm{IC}}=\sqrt{G_{\mathrm{C}} E}$. The value of the weight function is approximated by

$$
\alpha\left(F_{\xi}^{-1}(1-p)\right)=\alpha(a)=1.12 .
$$

Table 8.8 Parameter values for numerical examples.

\begin{tabular}{ll}
\hline Parameter & Value \\
\hline$\nu$ & 0.3 \\
$E$ & $10^{9} \mathrm{~Pa}$ \\
$m$ & $0.08 \mathrm{~kg} / \mathrm{m}^{2}$ \\
$h$ & $10^{-4} \mathrm{~m}$ \\
$l$ & $0.1 \mathrm{~m}$ \\
$b$ & $5 \mathrm{~m}$ \\
$G_{\mathrm{C}} / \rho$ & $10 \mathrm{Jm} / \mathrm{kg}$ \\
\hline
\end{tabular}

In Figs. 8.12 and 8.13 we illustrate the effect of changing the value of the distribution parameters and the admissible probability of fracture $p$ on the optimal values, when the crack length is assumed to obey the log-normal distribution. In Fig. 8.12 the optimal tension and the corresponding optimal velocity are plotted with respect to the distribution parameters $s_{1}$ and $c_{1}$ of the log-normal distribution. For this, it was set $p=0.001$.

In Fig. 8.13 we illustrate the effect of increasing the value of $p$ from $p=0.001$ with some values of the distribution parameters. As is expected, the optimal values increase when the admissible probability of fracture $p$ is increased. With smaller value of $s_{1}$, the change is small. 

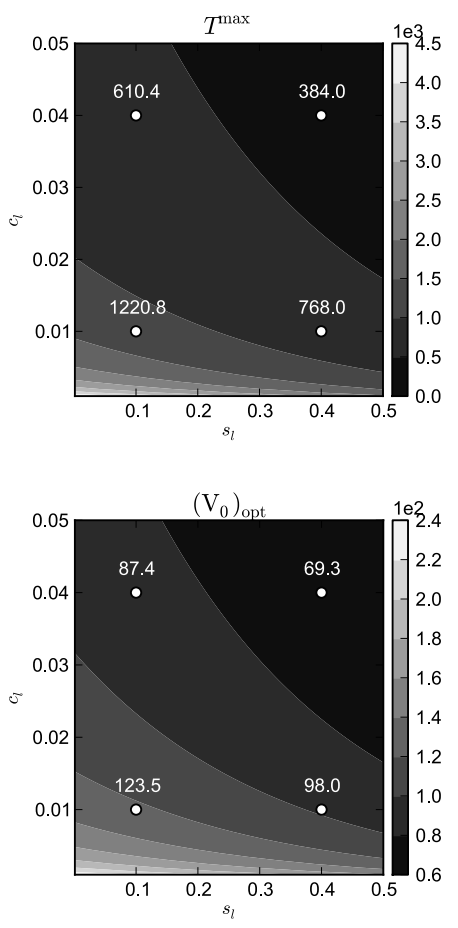

Fig. 8.12 Log-normal distribution. The effect of changing the value of the distribution parameters $s_{1}$ and $c_{1}$ on the optimal tension and velocity with the admissible probability of fracture $p=0.001$. 

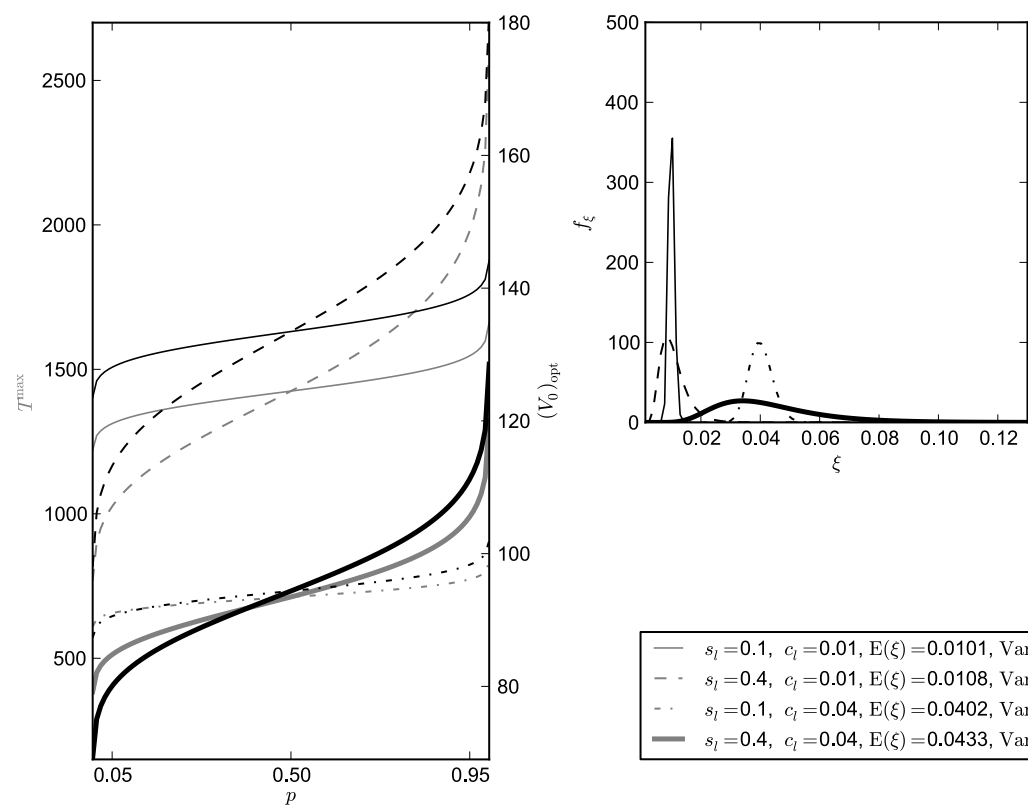

Fig. 8.13 Left: The effect of changing the admissible probability of fracture $p$ on the optimal values of tension and velocity, using a log-normal distribution for the crack length $\xi$. Right: the probability density function of $\xi$.

Table 8.9 The optimal tension (upper value, $\mathrm{N} / \mathrm{m}$ ) and velocity (lower value, $\mathrm{m} / \mathrm{s}$ ) with respect to $p$ and the distribution parameters $s_{1}$ and $c_{1}$, using a log-normal distribution for the crack length.

\begin{tabular}{lllll}
\hline & & \multicolumn{3}{c}{$p$} \\
\cline { 3 - 5 }$s_{1}$ & $c_{1}$ & 0.001 & 0.005 & 0.01 \\
\hline \multirow{2}{*}{0.1} & \multirow{2}{*}{0.01} & $1,220.8$ & $1,252.6$ & $1,268.3$ \\
& & 123.5 & 125.1 & 125.9 \\
& \multirow{2}{*}{0.04} & 610.4 & 626.3 & 634.2 \\
& & 87.4 & 88.5 & 89.0 \\
& & & & \\
\hline
\end{tabular}

In Fig. 8.14 we see the optimal tension and the corresponding optimal velocity plotted with respect to the distribution parameter $c_{\mathrm{t}}$ of the truncated normal distribution. The optimal values are computed with the initial crack length $a=0.05$. With the considered parameter values the function

$$
F_{\theta}\left(\frac{h K_{\mathrm{IC}}}{\alpha(a) \sqrt{\pi a}}-T_{0}\right)+q_{1}-1
$$


was found to be strictly decrasing with respect to $T_{0}$. The maximal value of tension was thus found by solving the equation

$$
F_{\theta}\left(\frac{h K_{\mathrm{IC}}}{\alpha(a) \sqrt{\pi a}}-T_{0}\right)+q_{1}-1=0 .
$$

Figure 8.14 shows that the more the tension is dispersed, the lower the optimal values are. Increasing the admissible probability of fracture or instability increases the optimal values. When the length of the initial crack increases, the optimal values decrease.

Some of the optimal values are gathered in Table 8.10.

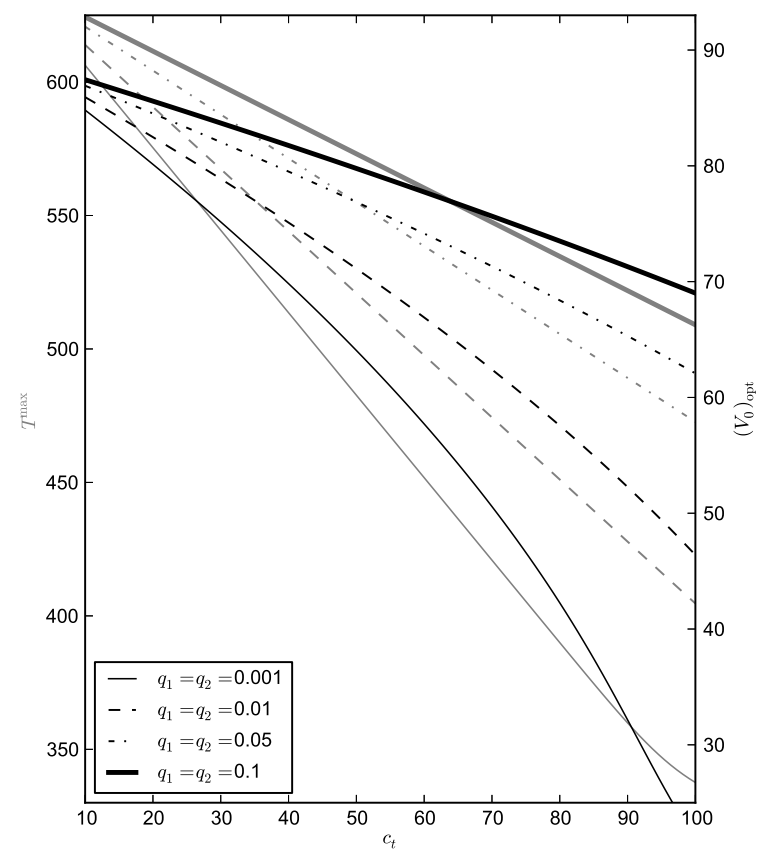

Fig. 8.14 Optimal tension and velocity, using the truncated normal distribution to model tension variation. The optimal values are shown with respect to the distribution parameter $c_{\mathrm{t}}$, in all cases using a constant crack length $a=0.05$. Here $q_{1}$ and $q_{2}$ are, respectively, the admissible probabilities of fracture and instability. 
Table 8.10 Truncated normal distribution. Optimal tensions (upper value, $\mathrm{N} / \mathrm{m}$ ) and velocities (lower value, $\mathrm{m} / \mathrm{s}$ ) with respect to the probabilities $q_{1}$ and $q_{2}$ and the distribution parameter $c_{\mathrm{t}}$. The crack length $a=0.05$.

\begin{tabular}{llll}
\hline & \multicolumn{3}{c}{$q_{1}, q_{2}$} \\
\cline { 2 - 4 }$c_{t}$ & 0.001 & 0.005 & 0.01 \\
\hline \multirow{2}{*}{10.0} & 606.3 & 611.4 & 613.9 \\
& 84.8 & 85.6 & 85.9 \\
50.0 & 482.7 & 508.4 & 520.9 \\
& 64.1 & 68.9 & 71.1 \\
100.0 & 337.6 & 380.1 & 404.6 \\
& 21.8 & 39.2 & 46.4 \\
& & & \\
\hline
\end{tabular}

To conclude, in this section we discussed the problem of finding the optimal velocity for an axially moving elastic plate with a crack from a probabilistic point of view. The model was assumed to include randomness, and the optimal velocity was investigated under the constraint that the probability of fracture is limited. Two cases were considered separately. First, the length of the crack was modelled as a random variable. Secondly, the in-plane tension was assumed to be a random variable. In the latter case, we also formulated a constraint for stability.

The optimal velocity was found by first computing the optimal tension for the plate. Assuming the crack length to be random, we formulated an analytical expression for the optimal tension. Modelling the crack length with the log-normal distribution, the effect of changing the admissible probability of fracture was numerically illustrated. It was seen that with both of the considered distributions, increasing the admissible probability of fracture increases the optimal tension and the corresponding optimal velocity.

The optimal velocity was also numerically computed for the log-normal distribution with different values of distribution parameters. The values were chosen such that the distributions were close to the presumable crack distribution of the paper making application.

The optimal tension and the related optimal velocity in the case of random in-plane tension were obtained, assuming the in-plane tension to obey a truncated normal distribution. It was seen that the more the tension is dispersed, the lower is the optimal velocity. Increasing the admissible probability of fracture had the same effect on the optimal values as in the case of random crack length. Also increasing the admissible probability of instability increased the optimal values. 


\section{References}

Banichuk N, Jeronen J, Neittaanmäki P, Tuovinen T (2010) On the instability of an axially moving elastic plate. International Journal of Solids and Structures 47(1):91-99, URL http://dx.doi.org/10.1016/j.ijsolstr.2009.09.020

Banichuk N, Kurki M, Neittaanmäki P, Saksa T, Tirronen M, Tuovinen $\mathrm{T}$ (2013) Optimization and analysis of processes with moving materials subjected to fatigue fracture and instability. Mechanics Based Design of Structures and Machines: An International Journal 41(2):146-167, URL http://dx.doi.org/10.1080/15397734.2012.708630

Banichuk NV, Neittaanmäki PJ (2010) Structural Optimization with Uncertainties. Springer-Verlag, Dordrecht, ISBN 978-90-481-2517-3

Björklund KJ, Svedjebrant J (2009) Productivity improvements of a newsprint paper machine by reduction of web breaks. Master's thesis, Luleå University of Technology

Chankong V, Haimes YY (1983) Multiobjective Decision Making Theory and Methodology. Elsevier Science Publishing Co., Inc., New York

Fett T (1999) Stress intensity factors for edge-cracked plates under arbitrary loading. Fatigue \& Fracture of Engineering Materials \& Structures 22(4):301-305, URL http://dx.doi.org/10.1046/j.1460-2695.1999.00156.x

Laham SA (1998) Stress Intensity Factor and Limit Load Handbook. British Energy Generation Ltd

Miettinen K (1994) On the methodology of multiobjective optimization with applications. Report 60, Department of Mathematics, University of Jyväskylä, ISBN 951-34-0316-5

Niskanen K (ed) (2012) Mechanics of Paper Products. Walter de Gruyter GmbH \& Co., ISBN 978-3-11-025461-7

Paris PC, Erdogan F (1963) A critical analysis of crack propagation laws. Journal of Basic Engineering, Transactions of the American Society of Mechanical Engineers D 85:528-534

Perez N (2004) Fracture Mechanics. Kluwer Academic Publishers

Salminen L (2003) Aspects of fracture processes in paper. PhD thesis, Helsinki University of Technology

Seth RS, Page DH (1974) Fracture resistance of paper. Journal of Materials Science 9(11):1745-1753, URL http://dx.doi.org/10.1007/BF00541741

Smith RD (1995) Roll and web defect terminology. TAPPI Press

Stadler W (1979) A survey of multicriteria optimization or the vector maximum problem, part I: 1776-1960. Journal of Optimization Theory and Applications 29(1):1-52

Steuer RE (1986) Multiple Criteria Optimization: Theory, Computation, and Applications. John Wiley \& Sons, Inc.

Uesaka T (2004) Web Breaks in Pressroom: A Review. FSCN-rapport, FSCN, Mitthögskolan, URL http://books.google.fi/books?id=xs6YMQAACAAJ 
Wathén R (2003) Characterizing the influence of paper structure on web breaks. Licentiate thesis, Helsinki University of Technology, Department of Forest Products Technology, espoo, Finland

White DJ (1990) A bibliography on the applications of mathematical programming multiple-objective methods. Journal of the Operational Research Society 41(8):669-691 



\section{Index}

acceleration

centripetal, 27, 84

Coriolis, 27, 84

local, 27,84

added-mass approximation, 127, 149, 153

generalized, 153

qualitative behaviour of solutions using, 153

added-mass model, 149, 193

aerodynamic

kernel, 143, 145, 150, 153, 160

problem, 127, 137

reaction, 15, 37, 128, 129, 150, 153, 156, 157

analytical solution of, 143

aeroelasticity, 138

airfoil theory, see thin airfoil theory

Airy stress function, 32, 85

for orthotropic material, 32

antisymmetric flow, 140

axial motion, 23

beam, 25,36

viscoelastic, 39, 106

bending

moment, 25, 106

operator, $27,43,59,85$

rigidity, $24,27,49,128,178,216,233$, 256

dimensionless (for panel), 155, 170, 173-175

dimensionless (for plate), 60, 93

orthotropic, $27,43,60,93$

biharmonic equation, 32,85

bilinear form, 61

boundary conditions, $22,28,31,38,44,45$, $85-87,92,108,111,158$ built-in, see clamped

$\mathrm{C}^{+}$-C, 109,123

$\mathrm{C}^{+}-\mathrm{S}, 109,123$

clamped, 25, 107

for airflow, 131, 135, 137-139

for Airy stress function, 33, 86

free of tractions, $22,26,28,50,61,85$, 92,129

hinged, see simply supported pinned, see simply supported SFSF, 22, 129

simply supported, $22,25,26,28,29,85$,

$129,130,158$

boundary layer theory, 150

brittle fracture condition, 218

buckling, 35

buckling mode, 49, 50, 59, 75, 77, 97, 99, 168,189

Cauchy problem, 164

Cauchy's principal value, 140, 141

Cauchy-Lagrange integral, 128

Cauchy-Riemann equations, 139

centripetal acceleration, 27, 84

classification of instabilities, 41

compatibility relation, 31

complex analysis, 128, 135, 139

complex-valued solution, 40, 159

condition for non-trivial solutions, see zero determinant condition

condition for regularity (potential flow), 140

Coriolis acceleration, 27, 84

crack growth theory, fatigue, 234

crack growth, fatigue, 221, 231

crack, edge, 215, 217, 220

cracked elastic plate, 254 
creep (viscoelasticity), 103

criterion functions, 246

critical eigenmode, 169

critical fracture toughness, 256, 257

critical velocity, $42,49,53,66,75,76,97$, 99, 112, 115-117, 155, 168, 170-172, 175,191

cumulative distribution function, 257, 258, 260

cyclic in-plane tension, 221, 231

cylindrical rigidity, see bending rigidity

deflection, see displacement

determinant method, 191

diagonalization method, 181, 190

differential operator, linear, 40

dimensionless retardation time, 125

Dirac delta distribution, 149

displacement, 133

in-plane, 31, 133

out-of-plane, $22,24,25,43,85,128,133$, 153,157

disturbance potential, 128, 136, 139

divergence instability, 36, 37, 41, 42, 49, $50,59,83,112,115,164,216$

divergence mode, 49, 50, 59, 75, 77, 97, 99, 168,189

dominated convergence, 147

Duxbury distribution, 255

dynamic instability, see flutter instability

dynamic stability analysis, 35

dynamical behaviour problem, 128, 154, $156,158,159,164,180$

edge crack, 215, 217, 220

eigenfrequency

analysis, 39, 159, 190, 198

problem, 154,158, 159, 164, 190, 192

spectrum, 191, 193, 197, 198, 211

eigenfunction, see eigenmode

eigenmode, 40, 50, 60, 91, 93, 160, 173

eigenvalue problem, 44, 49, 50, 60, 91, 93, 114,216

generalized, linear, 167

quadratic, 114

eigenvalues, non-negativeness of, 61

elastic moduli, 28,30

equilibrium of stresses, $30,31,86$

Euler coordinates, see Eulerian (stationary) frame of reference, see Eulerian (stationary) frame of reference

Euler derivative, 22

Euler value of critical force, 56
Eulerian (stationary) frame of reference, $14,22,131,133$

fatigue crack growth, 221,231

fatigue crack growth theory, 234

finite difference method, 94, 112

finite element method, 138

flexural stiffness, 25

flow, antisymmetric, 140

fluid mechanics, 13

fluid-structure interaction, 37, 127, 149

flutter instability, 41, 42, 115, 197

Fourier basis, 181

Fourier sine basis, 160

Fourier-Galerkin method, 159, 197

fracture, 215

condition, brittle, 218

mechanics, linear elastic, 215

toughness, 218

critical, 256, 257

of paper, $224,239,251,260$

frame of reference

Eulerian (stationary), 14, 22, 131, 133

Lagrangean (co-moving), 14, 23, 133

free-stream potential, 128, 136

Fubini's theorem, 163

Galerkin method, 159

Galerkin series, 159, 161, 180

Galilean relativity, 14

generalized added-mass approximation, 153

generalized Hooke's law, 30, 105

inverse of, 32

geometric average shear modulus, 29, 63, $68,77,92$

geometric factor, 218, 235

Green's 2nd identity, 46

Green's function, 128, 145, 153

group velocity, of waves, 23

gyroscopic system, 191

Hamilton's principle, 107

harmonic function, 135

Hermitian conjugate, 192

homogeneous tension, 30, 43-45, 49, 59, $216,233,256$

Hooke's law, generalized, 30, 105

inverse of, 32

in-plane

displacement, 31, 133

stress, 27

tension, $29,85,231$ 
initial conditions, 158

instability

classification of, 41

divergence, $36,37,41,42,49,50,59,83$, $112,115,164,216$

dynamic, see flutter

flutter, 41, 42, 115, 197

static, see divergence

integrodifferential equation, 127

irrotational flow, 137

isotropic plate, $21,27,28,45,46,49,83$, $216,221,256$

Kelvin-Voigt model, 103-105

Kronecker delta, 162

Kutta-Zhukowski condition, 137

Lagrange coordinates, see Lagrangean (co-moving) frame of reference

Lagrange derivative, see material derivative

Lagrangean (co-moving) frame of reference, $14,23,133$

Laplace's equation, 135, 145

least squares fit, 150, 193

linear differential operator, 40

linear elastic fracture mechanics, 215

linear stability analysis, 40

linear-logarithmic search procedure, 168

linearized model, 42

linearly distributed tension, 33, 86, 219, 220

local acceleration, 27,84

log-normal distribution, 255, 258

longevity, 222, 231, 251

mass lumping, 150

material derivative, $22,39,131$

material, moving, 13

membrane, 21, 27, 36, 44

membrane operator, 27,84

Monte Carlo method, 149

motion, axial, 23

moving material, 13

multi-objective

optimization, 243

problem, 245, 251

programming, see optimization

Newton's second law, 107

non-homogeneous tension, 32, 84

non-negativeness of eigenvalues, 61

non-tensioned panel, 166, 176

objective functional, 245 open draw, 14,16

operator

bending, 27, 43, 59, 85

differential, linear, 40

differential, of vacuum problem, 155

integro-differential, of fluid-structure interaction, 155

membrane, 27, 84

optimal productivity, 243

optimization

multi-objective, 243

problem, 245, 251

optimization problem, 231, 239, 257

orthotropic plate, $21,27,28,38,46,58,76$

out-of-plane

displacement, 22, 24, 25, 43, 85, 128, $133,153,157$

vibration, $21,22,24,25,36,43,84,87$, 157

panel, 21, 24, 127, 129-131, 137, 154, 157, 178,197

non-tensioned, 166, 176

viscoelastic, 108, 109, 111, 115, 118-123

paper machine, 14, 15, 17

generation of axial tension in, 133, 258

paper production, 13-15, 231

paper web, 14

Pareto front, 247, 248, 250

Pareto optimal solution, 243, 246

Paris constant, 240

performance function, 233

perturbed vacuum model, 165

plate, $21,25,26,129,217,232$

isotropic, $21,27,28,45,46,49,83,216$, 221,256

orthotropic, 21, 27, 28, 38, 46, 58, 76

Poisson ratio, 28, 128

potential flow, 127, 135, 137, 139, 153, 154, 178, 197

memory-free property of, 139, 154

potential flow theory, 137

potential, of velocity, 128, 135

disturbance, 128, 136, 139

free-stream, 128, 136

process effectiveness criterion, 248

productivity criterion, 233, 234

productivity function, 239

pseudo-steady-state problem, 158

quadratic eigenvalue problem, 114

quasistatic loading process, 221

real-valued solution, 40, 159 
reduction

narrow strip to $1 \mathrm{D}, 56$

orthotropic to isotropic, 29, 92

to first-order system, 180

regularity condition (potential flow), 140

relaxation (viscoelasticity), 103

Riemann's mapping theorem, 137

roller symbol, 24, 26, 129, 130

for string, 23

shear modulus, geometric average, 29, 63, $68,77,92$

singular value decomposition, 191

solution

complex-valued, 40, 159

real-valued, 40, 159

steady-state, 42, 50, 60, 91, 155, 165

spectral boundary value problem, 50

spectral method, 159

speed of propagation, waves, 23

stability analysis, 35, 43, 215

dynamic, 35, 43

linear, 40

static, 35, 49, 59, 91, 155, 156, 164

stability exponent, 41, 45, 87, 111

static instability, see divergence instability

static stability analysis, 35, 49, 59, 91, 155, 156,164

static stability problem, 49, 59, 91, 154-156, 164

steady-state problem, 159, 164, 167

steady-state solution, 42, 50, 60, 91, 155, 165

stochastic analysis, 254

strain compatibility equation, 32

strain energy, 61

strain energy release rate, 224

strain-displacement relations, 30

stress equilibrium equations, 30, 31, 86

stress intensity factor, 217, 222, 235, 256

stress, in-plane, 27,85 stress-strain relations, 104

string, 21, 22, 36

tension, in-plane, 29, 85

cyclic, 221, 231

homogeneous, 30, 43-45, 49, 59, 216, 256

linearly distributed, 33, 86, 219, 220

non-homogeneous, 32,84

thin airfoil theory, 128, 150, 153

threadline, 22

time-harmonic trial function, 40, 43, 87, $110,158,190$

time-harmonic vibration, 41, 44, 45

transverse displacement, see out-of-plane displacement

transverse vibration, see out-of-plane

vibration

truncated normal distribution, 260, 264

velocity-dependent phase shift, 182

vibration

out-of-plane, $21,22,24,25,36,43,84$, 87,157

time-harmonic, 41, 44, 45

viscoelastic

beam, 39, 106

material, 38, 103, 105

panel, 108, 109, 111, 115, 118-123

vortex panel method, 138

wave equation, 22

weak form, 160

web, paper, 14

Weibull distribution, 255

weighting factor, 245

weighting method problem, 248

Young's modulus, 25, 28, 128

zero determinant condition, 51, 64 